\title{
NMR Characterizations of Properties of Heterogeneous Media
}

\author{
Final Report \\ for the period
}

May 1999 - December 2004

\author{
C. T. Philip Chang ${ }^{\dagger}$, Changho Choi ${ }^{\dagger}$, Jeromy T. Hollenshead ${ }^{\dagger}$, \\ Rudi Michalak*, Jack Phan*, Ramon Saavedra*, John C. Slattery ${ }^{\dagger}$, \\ Jinsoo $\mathrm{Uh}^{* \dagger}$, Randi Valestrand*†§, A. Ted. Watson*, and Song Xue*† \\ *Department of Chemical Engineering, Colorado State University \\ Fort Collins, CO 80526-2039 \\ $\dagger$ Department of Chemical Engineering, Texas A\&M University \\ College Station, Texas 77843-3122 \\ $\S$ Department of Physics, University of Bergen \\ Bergen, Norway
}

January, 2005

DOE Award Number DE-AC26-99BC15202

Texas Engineering Experiment Station (TEES)

Texas A\&M University

College Station, Texas 77843-3122 


\section{DisClAimeR}

This report was prepared as an account of work sponsored by an agency of the United States Government. Neither the United States Government nor any agency thereof, nor any of their employees, makes any warranty, express or implied, or assumes any legal liability or responsibility for the accuracy, completeness, or usefulness of any information, apparatus, product, or process disclosed, or represents that its use would not infringe privately owned rights. Reference herein to any specific commercial product, process, or service by trade name, trademark, manufacturer, or otherwise does not necessarily constitute or imply its endorsement, recommendation, or favoring by the United States Government or any agency thereof. Their views and opinions of authors expressed herein do not necessarily state or reflect those of the United States Government or any agency thereof. 


\begin{abstract}
A critical and long-standing need within the petroleum industry is the specification of suitable petrophysical properties for mathematical simulation of fluid flow in petroleum reservoirs (i.e., reservoir characterization). The development of accurate reservoir characterizations is extremely challenging. Property variations may be described on many scales, and the information available from measurements reflect different scales. In fact, experiments on laboratory core samples, well-log data, well-test data, and reservoir-production data all represent information potentially valuable to reservoir characterization, yet they all reflect information about spatial variations of properties at different scales.

Nuclear magnetic resonance (NMR) spectroscopy and imaging (MRI) provide enormous potential for developing new descriptions and understandings of heterogeneous media. NMR has the rare capability to probe permeable media non-invasively, with spatial resolution, and it provides unique information about molecular motions and interactions that are sensitive to morphology. NMR well-logging provides the best opportunity ever to resolve permeability distributions within petroleum reservoirs.

We develop MRI methods to determine, for the first time, spatially resolved distributions of porosity and permeability within permeable media samples that approach the intrinsic scale: the finest resolution of these macroscopic properties possible. To our knowledge, this is the first time that the permeability is actually resolved at a scale smaller than the sample.

In order to do this, we have developed a robust method to determine of relaxation distributions from NMR experiments and a novel implementation and analysis of MRI experiments to determine the amount of fluid corresponding to imaging regions, which are in turn used to determine porosity and saturation distributions. We have developed a novel MRI experiment to determine velocity distributions within flowing experiments, and developed methodology using that data to determine spatially resolved permeability distributions.

We investigate the use of intrinsic properties for developing improved correlations for predicting permeability from NMR well-logging data and for obtaining more accurate estimates of multiphase flow properties-the relative permeability and capillary pressure-from displacement experiments. We demonstrate the use of MRI measurements of saturation and relaxation for prediction wetting-phase relative permeability for unstable experiments. Finally, we developed an improved method for determining surface relaxivity with NMR experiments, which can provide better descriptions of permeable media microstructures and improved correlations for permeability predictions.
\end{abstract}




\section{Contents}

1 Introduction $\quad 3$

2 Background $\quad 4$

2.1 Modeling flow in permeable media . . . . . . . . . . . . . . . . . . 4

2.2 Determination of properties $\ldots \ldots \ldots \ldots \ldots$

2.3 Prediction of properties $\ldots \ldots \ldots \ldots \ldots$

3 Determination of NMR Relaxation Distributions $\quad 9$

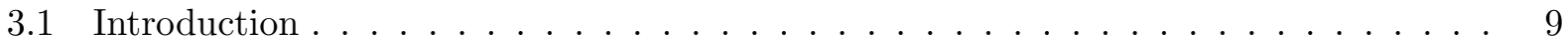

3.2 Modeling NMR relaxation distributions f . . . . . . . . . . . . . . . . . 11

3.3 Statistical selection of regularization parameter . . . . . . . . . . . . . . . 14

3.4 Validation with simulated data . . . . . . . . . . . . 16

3.5 Analysis with experimental data . . . . . . . . . . . . . . . 25

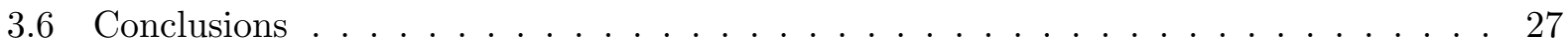

4 Determination of Surface Relaxivity and Pore-Size Distributions 29

4.1 Introduction . . . . . . . . . . . . . . . . . . . . . . 29

4.2 Scaling relaxation distribution to pore-size distribution . . . . . . . . . . . . 29

4.3 Determination of surface relaxivity using PFGSTE experimental data . . . . . . . 30

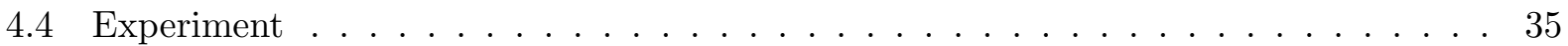

4.5 Results and discussion . . . . . . . . . . . . . . . . . 35

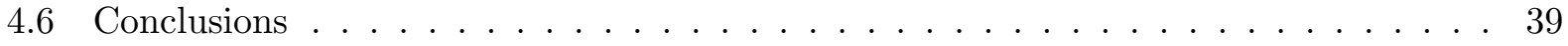

5 Determination of Porosity and Saturation Distributions 40

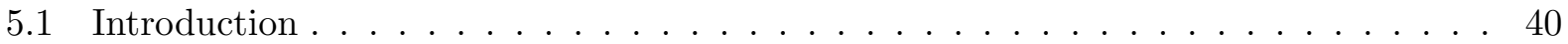

5.2 Methodology ........................... 41

5.3 Experimental results and discussion $\ldots \ldots \ldots \ldots$. . . . . . . . . . . . 42

5.4 Conclusions . . . . . . . . . . . . . . . . . . . . . 44

6 Determination of Permeability Distributions using Velocity Imaging Experi$\begin{array}{ll}\text { ments } & 47\end{array}$

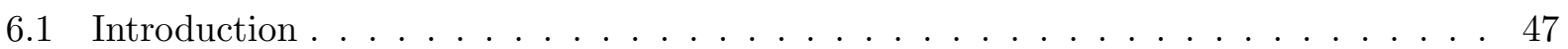

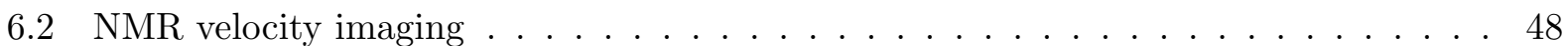


6.2 .1 Methodology . . . . . . . . . . . . . . . . . 48

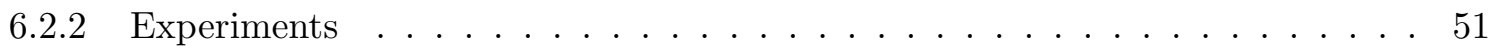

6.2 .3 Results and discussion . . . . . . . . . . . . . . . . . . 54

6.3 Determination of permeability distributions . . . . . . . . . . . 61

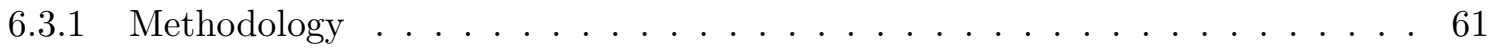

6.3 .2 Validation with simulated data . . . . . . . . . . . . . . . 64

6.3.3 Analysis with experimental data . . . . . . . . . . . . . . 69

6.3 .4 Improved experimental design . . . . . . . . . . . . . . . . 72

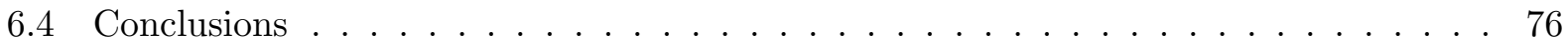

$\begin{array}{lll}7 & \text { Determination of Multiphase Flow Properties } & 77\end{array}$

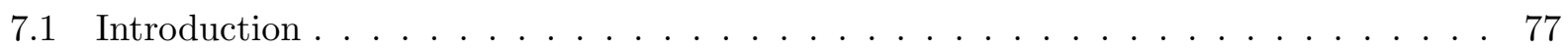

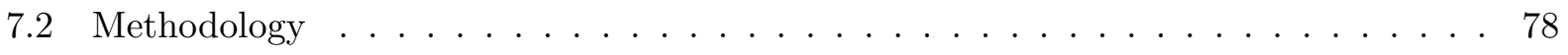

7.3 Value of including intrinsic permeability . . . . . . . . . . . 80

7.4 Displacement experiments . . . . . . . . . . . . . . 86

7.4 .1 Experimental setup . . . . . . . . . . . . 86

7.4 .2 Experimental design . . . . . . . . . . . . . . 87

7.4 .3 Results and discussion . . . . . . . . . . . . . . . 90

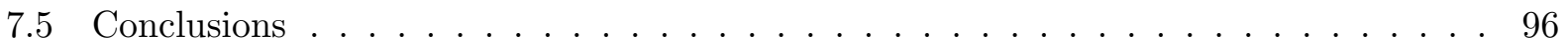

8 Prediction of Properties $\quad 97$

8.1 Introduction . . . . . . . . . . . . . . . . . . . 97

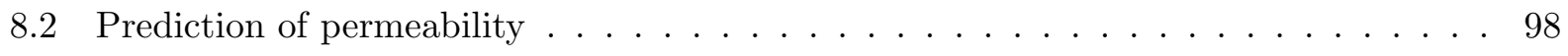

8.2 .1 Permeability correlations . . . . . . . . . . . . . 99

8.2 .2 Predictions with intrinsic properties . . . . . . . . . . . 100

8.3 Prediction of relative permeability $\ldots \ldots \ldots \ldots$

8.3 .1 Experiment . . . . . . . . . . . . . . . . 107

8.3 .2 Results and discussion . . . . . . . . . . . . . . . . 108

9 Conclusions $\quad 118$

$\begin{array}{lr}\text { References } & 120\end{array}$

$\begin{array}{lr}\text { List of Acronyms and Abbreviations } & 128\end{array}$ 
Appendices 


\section{List of Figures}

1 "True" relaxation distribution $\ldots \ldots \ldots \ldots \ldots$

2 Simulated data. (a) $0.1 \%$ noise, (b) $1.0 \%$ noise, (c) $5.0 \%$ noise . . . . . . . . . . 20

3 CV scores used for determination of the optimal $\lambda$. The $5.0 \%$ noise data are used.

Figure (a) is zoomed in figure (b) with smaller axes scales . . . . . . . . . . . 21

4 The integral-square-difference (ISD) of $P_{N_{i}}(\tau)$ and $P_{N_{j}}(\tau)$ for $1.0 \%$ noise data using UBR criterion (a) ISD for the adjacent numbers of knots among those examined

(b) ISD with respect to the largest number of knots . . . . . . . . . . . . . 22

$5 \quad$ Estimated relaxation time distribution (a) $0.1 \%$ noise, (b) $1.0 \%$ noise, (c) $5.0 \%$ noise 23

6 Predicted relaxation curves compared with the simulated data (a) $0.1 \%$ noise, (b) $1.0 \%$ noise, (c) $5.0 \%$ noise . . . . . . . . . . . . . . . . . 24

$7 \quad T_{1}$ relaxation data by inversion-recovery experiment $\ldots \ldots \ldots \ldots$

8 NMR relaxation distributions of the Bentheimer sample $($ BENT1) $\ldots \ldots \ldots$

$9 \quad$ Procedure of the proposed method for determination of surface relaxivity . . . . . 32

10 The "13-interval, Condition I" pulsed field gradient stimulated echo pulse sequence . 35

11 PFGSTE data and calculated values . . . . . . . . . . . . . . 37

12 Determined pore-size distribution . . . . . . . . . . . . . . . 37

13 Apparent diffusivities and short-time approximation . . . . . . . . . . 38

14 An example of three-dimensional CPMG imaging pulse sequence. . . . . . . . . . . 41

15 The relaxation data (a) and the estimatied distributiion function (b) of a voxel at $Z_{1}=16, Z_{2}=9, Z_{3}=8$ in the Bentheimer sample (BENT1) . . . . . . . 44

16 Three-dimensional porosity distribution of the Bentheimer sample . . . . . . . . 45

17 Porosity histogram of the Bentheimer sample and the corresponding statistical analysis 46

18 An example of stimulated-echo pulse sequence for detecting spin displacements along $z$-direction. The time between the second and third RF pulses, TM, is referred to as

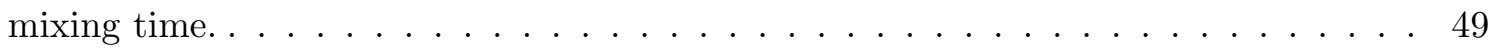

19 The "positioner" in which the RF coil is mounted. . . . . . . . . . . . . . . . . 52

20 Superficial average velocity of the thin Bentheimer sample (BENT2) . . . . . . . 54

21 An example of joint spin-velocity density function $\left(z_{1}\right.$-component, the coordinate numbers for the voxel are $Z_{1}=18, Z_{2}=3$ and $\left.Z_{3}=6\right) \ldots \ldots \ldots$

22 Superficial average velocity of the cylindrical Bentheimer sample . . . . . . . . . 55 
23 Velocity encoded stimulated echo data (a,c) and their joint spin-velocity density function $(b, d)$ of a voxel in the Bentheimer sample with flow rates $0 \mathrm{ml} / \mathrm{min}(\mathrm{a}, \mathrm{b})$ and $20 \mathrm{ml} / \mathrm{min}(\mathrm{c}, \mathrm{d}) \ldots \ldots \ldots \ldots \ldots \ldots \ldots$

24 Velocity encoded stimulated echo data (a,c) and their joint spin-velocity density function (b,d) of a voxel in R237 sample with flow rates $0 \mathrm{ml} / \mathrm{min}(\mathrm{a}, \mathrm{b})$ and 20

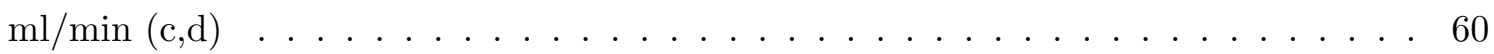

25 The domain of the fluid flowing system . . . . . . . . . . . . . . 62

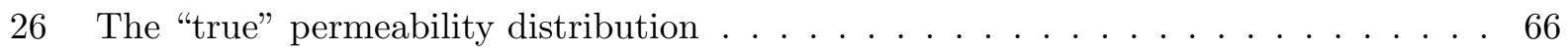

27 Simulated velocity distribution calculated with the "true permeability" . . . . . . 67

28 RMSE as a function of regularization parameter $\lambda \ldots \ldots \ldots \ldots \ldots$

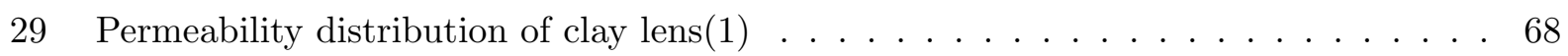

30 Determined permeability distribution using the velocity distribution in Fig. 20 . . 70

31 Determined permeability distribution using the velocity distribution in Fig. 22 . . 71

32 Examples of flow conditions . . . . . . . . . . . . . . . . . . . 74

33 True permeability distribution . . . . . . . . . . . . . . 74

34 Simulated velocity data . . . . . . . . . . . . . . . 75

35 Estimated permeability distribution with different experimental designs $\ldots . . .75$

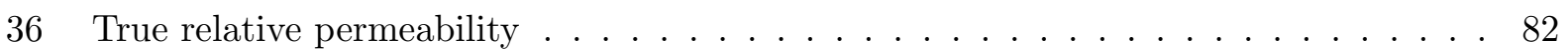

37 Capillary pressure curves used in the estimation process . . . . . . . . . . 82

38 Absolute permeability distributions used in the estimation . . . . . . . . . 83

39 Estimated relative permeability functions for two cases . . . . . . . . . . . . 83

40 Simulated pressure drop data and the calculated values for Case $1 \ldots$. . . . . . 84

41 Simulated pressure drop data and the calculated values for Case $2 \ldots \ldots 4$

42 Simulated saturation data and the calculated values for Case $1 \ldots \ldots$. . . . . . 85

43 Simulated saturation data and the calculated values for Case $2 \ldots \ldots$

44 Schematic of experimental apparatus for displacement experiments . . . . . . . . 86

45 Sample holder device . . . . . . . . . . . . . . . . 87

46 Schematic of the sample as it resides within the RF-coil inside the MRI bore . . . 89

47 Pressure drop data of the primary drainage cycle of Experiment III . . . . . . . . . 91

48 One-dimensional first echo image for Experiment III . . . . . . . . . . . . . . . 92

49 The signal intensity of bulk fluid within the inlet and outlet endcaps for Experiment II 92

50 Saturation of nonwetting phase for 1st cycle of Experiment III $(0.3 \mathrm{ml} / \mathrm{min}) \ldots . . .94$ 
51 Saturation of nonwetting phase for 1st cycle of Experiment III $(0.6 \mathrm{ml} / \mathrm{min}) \ldots \ldots 4$

52 Saturation of nonwetting phase for 1st cycle of Experiment III $(0.9 \mathrm{ml} / \mathrm{min})$. . . . 95

53 Saturation of nonwetting phase for 1st cycle of Experiment III $(1.2 \mathrm{ml} / \mathrm{min})$. . . . 95

54 Permeability of the voxels in the Bentheimer sample versus its predictor, $a \phi^{b}\left(T_{2 l m}\right)^{c} .103$

55 Sample orientation with imaging experiments . . . . . . . . . . . 108

56 Stage 1 saturation distributions for two successive horizontal planar regions near the center of the sample . . . . . . . . . . . . . . . . . . 110

57 Stage 2 saturation distributions for two successive horizontal planar regions near the center of the sample . . . . . . . . . . . . . . . . . 111

58 Stage 3 saturation distributions for two successive horizontal planar regions near the center of the sample . . . . . . . . . . . . . . . . . 111

59 Stage 1 saturation distributions for a horizontal planar region near the center of the

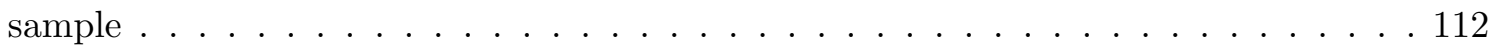

60 Stage 2 saturation distributions for a horizontal planar region near the center of the

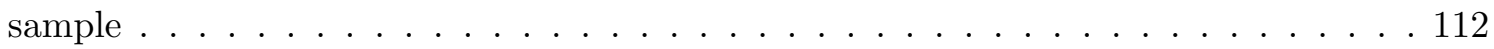

61 Stage 3 saturation distributions for a horizontal planar region near the center of the

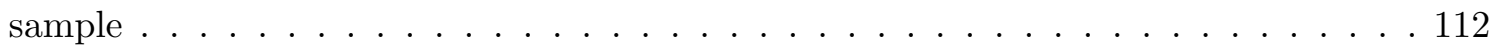

62 Stage 1 Voxel saturation values along the axial direction . . . . . . . . . . . 113

63 Stage 2 Voxel saturation values along the axial direction . . . . . . . . . . . . 113

64 Stage 3 Voxel saturation values along the axial direction . . . . . . . . . . . . 113

$65 T_{1}$ distributions for $\operatorname{Voxel}(35,7,6) \ldots \ldots \ldots \ldots \ldots \ldots \ldots$

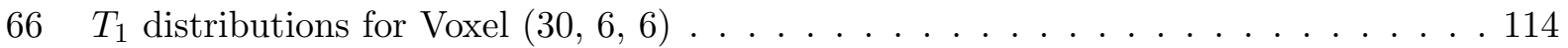

67 Average relaxation for different saturations for two voxels . . . . . . . . . . 116

68 Average relaxation for different saturations for sixty voxels $\ldots \ldots \ldots 117$

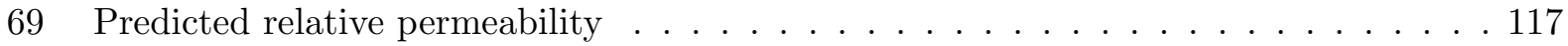

$70 \quad$ NMR relaxation distributions of $\mathrm{R} 138 \ldots \ldots \ldots \ldots \ldots$

71 NMR relaxation distributions of R145 . . . . . . . . . . . . . . . 132

72 NMR relaxation distributions of $\mathrm{R} 221 \ldots \ldots \ldots \ldots$

73 NMR relaxation distributions of $\mathrm{R} 237 \ldots \ldots \ldots \ldots \ldots$

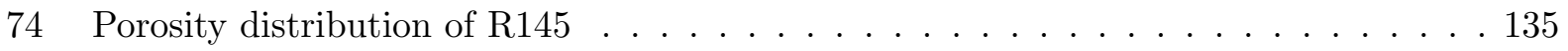

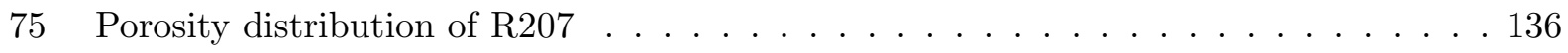

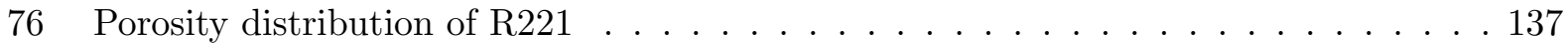




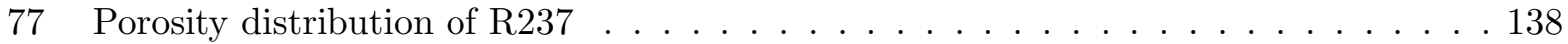




\section{List of Tables}

1 The results of the estimation using 300 B-spline knots $\ldots \ldots \ldots$

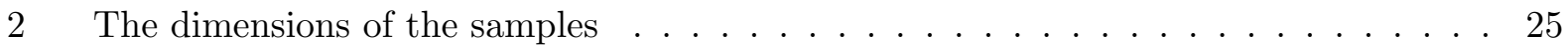

3 Determined average relaxation times of the samples $\ldots \ldots \ldots \ldots$

4 Results of surface relaxivity estimation for reservoir samples . . . . . . . . . . 36

$5 \quad$ Results of surface relaxivity estimation for reservoir samples (The experimental data for

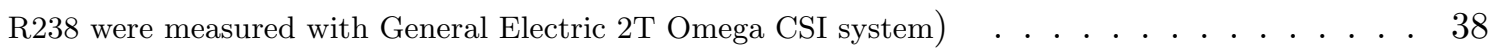

6 Parameters of CPMG imaging sequences . . . . . . . . . . . . . . 43

$7 \quad$ Statistical analysis on porosity distributions of various samples $\ldots \ldots$. . . . . . . 45

8 Pulse sequence parameters used in velocity imaging . . . . . . . . . . . 53

$9 \quad$ Typical attenuation of the samples $(T E=7.5 \mathrm{~ms}$ and $T M=200 \mathrm{~ms}) \ldots \ldots 7$

10 RMSE of the calculated velocity and the estimated permeability distribution . . . 74

11 Design of displacement experiments (The time in parenthesis indicates when the corresponding flow rate was started based from the beginning of the experiment) . . 88

12 Basic properties of fluids $\left(\right.$ at $\left.18{ }^{\circ} \mathrm{C}\right) \ldots \ldots \ldots \ldots$

13 Results of linear regression for the parameters of correlation equation . . . . . . . . 102

14 Correlation coefficients for various realizations. The three parameters, $\log a, b$, and $c$ were estimated by linear regression and the corresponding $k^{\text {pred }}$ was calculated. . . 104

15 Predicted apparent permeability using the determined correlation equations. . . . . 105

16 Parameter values for CPMG imaging and inversion-recovery sequences . . . . . . . 109

17 Range of voxels examined . . . . . . . . . . . . . . . . . . 109

18 Average relaxation and saturation values for two voxels $\ldots \ldots \ldots \ldots$ 


\section{Executive Summary}

Nuclear magnetic resonance (NMR) spectroscopy and imaging (MRI) provide exceptional opportunity for developing new descriptions and understandings of heterogeneous media. NMR has the rare capability to probe permeable media non-invasively, with spatial resolution, and it provides unique information about molecular motions and interactions that are sensitive to morphology. NMR well-logging provides the best opportunity ever to resolve permeability distributions within petroleum reservoirs.

The overall goal of this project was to fully develop MRI methods for improved characterizations of properties used to describe flow through permeable media. This work was organized along three stages. The first stage was to develop advanced core analysis methods for fully characterizing complete porosity and absolute permeability distributions, saturation distributions, and relative permeability and capillary pressure functions from laboratory samples. The second stage was to use these methods to resolve properties on a set of samples. The third stage was to develop and test improved methods for predicting permeability, and test a novel method for predicting relative permeability, from well-log observable data.

We developed methodology to fully resolve spatial distributions of the porosity and absolute permeability, and saturations for two-phase situations, within samples of permeable media and to account for spatial variations in permeability and porosity for the determination of relative permeability and capillary pressure functions. We believe this provides a major breakthrough for the study of heterogeneous media in that, for the first time, we can resolve all the basic properties at, or near, the most fundamental (intrinsic) scale for macroscopic properties: the minimum local volumeaveraging element. Previous attempts to understand or predict properties to describe heterogeneous media have been based on the core sample as the most fundamental element, with the assumption that the associated properties within are uniform (homogeneous).

We applied these methods to experiments with samples of Bentheimer sandstone and to actual sandstone reservoir samples provided by a major petroleum company. We resolved the porosity distributions and, with multiphase experiments performed on a subset of the samples, saturation distributions. We determined the permeability distributions for the Bentheimer sandstone samples. Due to the fast relaxation rates for water saturating the sandstone reservoir samples, we were unable to resolve the velocity distributions with MRI, and thus were unable to determine the permeability

distributions. Our method can be applied to such samples if a saturating fluid with sufficiently long 
relaxation time is identified. Also, the method should be successful with most carbonate samples, since the relaxation rates associated with water within such samples are typically smaller than sandstones.

We advanced the use of full, three-dimensional mathematical models for use in determining multiphase flow properties from displacement experiments. We recommend including intrinsic porosity and permeability, dynamic one-dimensional saturation profiles, and three-dimensional saturation distributions corresponding to steady states within the process to estimate multiphase flow properties. Using MRI, we have determined the boundary conditions conventionally used to model displacement experiments may not accurately match the experiments, and thus recommend future attention to this aspect. The problem was not solved in the course of this work.

We demonstrated the use of spatially resolved property distributions for determining improved correlations for predicting permeability, and demonstrated the use of spatially resolved saturation and relaxation distributions for predicting wetting-phase relative permeability.

Several additional projects, not identified within the original proposal, were taken up. While these projects all supported the original scope of the work, their necessity was recognized as we worked with actual reservoir samples. We developed a new method for determining relaxation distributions from NMR data, since existing methods were not sufficiently accurate or robust for analyzing three-dimensional imaging data. In addition to its use for determining saturation and porosity, this method will provide better estimates of pore-size distributions from NMR experiments. We developed a new method for estimating surface relaxivities with NMR experiments. Surface relaxivity will be important for developing better predictions of permeability. It is also necessary to determine pore-size distributions from relaxation distributions. We implemented an imaging method to measure spatially-resolved longitudinal relaxation $\left(T_{1}\right)$ distributions. This became necessary when we determined transverse relaxation $\left(T_{2}\right)$ distributions measured at high field would not suitable for developing predictive equations. Finally, we demonstrated the use of relative permeability prediction with unstable displacement experiments, and noted the potential use of MRI measurements of saturation distributions to advance the modeling and understanding of unstable displacements. 


\section{Introduction}

Reliable simulation of fluid flow in petroleum reservoirs is necessary for meaningful evaluations of production potentials, design of effective recovery strategies, and selection of production operations. A critical and long-standing need within the oil industry is the specification of suitable petrophysical properties for such simulations, i.e., reservoir characterization.

The development of accurate reservoir characterizations is extremely challenging. Petroleum reservoirs are heterogeneous so that properties required to simulate reservoir behavior vary spatially. This is further complicated by the fact that the property variations may be described on many scales, and the information available from measurements reflect different scales. In fact, experiments on laboratory core samples, well-log data, well-test data, and reservoir-production data all represent information potentially valuable to reservoir characterization, yet they all reflect information about spatial variation of properties at different scales.

We believe that the most valuable characterization of reservoir property heterogeneities begins at the most fundamental scale, we call the intrinsic scale. For porosity and permeability, this corresponds to the basic continuum scale associated with the existence of those properties, typically on the size of tens of hundreds of "pores". The intrinsic scale associated with multiphase flow properties may be somewhat larger, although accurate determination of those properties often depends upon knowledge of the intrinsic porosity and permeability. Traditionally, the finest resolution of properties has been at the reservoir core sample scale. This is insufficient for developing relationships that may link heterogeneous properties across scales. Furthermore, the determination of these properties may have significant errors associated with them, as discussed in the following section.

Nuclear magnetic resonance (NMR) spectroscopy and imaging (MRI) provide exceptional opportunity for improved characterization of fluid and flow in permeable media. MRI provides the rare opportunity for determining, non-invasively, spatially resolved data within permeable media. We develop methods utilizing MRI to determine, for the first time, porosity and permeability at

the intrinsic scale. We develop methodology to determine spatial distributions of fluid saturations for multiphase situations. We have also developed a method to determine multiphase flow properties (relative permeability and capillary pressure) for heterogeneous media, utilizing the intrinsic porosity and permeability so that errors associated with spatial variations in those properties can be avoided. We believe that resolution of these properties at the intrinsic scale can lead to: 1. 
improved understanding and mathematical models of complex displacement processes within heterogeneous media, 2. methods for scale-up that can provide effective properties at coarser scales that are suitable for reservoir simulation, 3. methods for reconciliation of data that represent different scales of heterogeneity, and 4. improved methods for predicting important properties from well-log measurements. This latter point is addressed further in this work.

NMR well logging provides the best opportunity ever to obtain reliable predictions of permeability and relative permeability in the near-well bore region. Unlike traditional well-logging measurements, NMR is sensitive to solid-liquid molecular interactions, and can thus provide information about media morphology, which controls permeability. Vertical resolution of permeability around wells can provide important opportunities for extrapolating property variations throughout the reservoir. Determination of the intrinsic properties, as well as spatial variations of NMRrelaxation, can provide the means for developing much more powerful correlations for property prediction. In this work, we demonstrate the use of intrinsic properties for developing correlations for predicting absolute and relative permeability. Finally, we demonstrate the use of MRI to determine predictions of relative permeability for unstable displacement processes, and to determine information that can be valuable for studying and simulating unstable processes.

\section{Background}

In this section, we develop the intrinsic scale associated with properties used to describe the flow of single or multiple fluid phases through permeable media. We summarize the basic approach required to determine media properties, and the principles for developing methods to predict properties from indirect observations.

\subsection{Modeling flow in permeable media}

To describe flow in permeable media, one could in principle use the well-established mass and momentum conservation equations, with constitutive equations relating velocity gradients and shear stresses, for the fluids within the pore space, together with associated boundary conditions at the solid/fluid interface. We refer to this as the "microscopic" approach. However, the actual geometry of permeable media essentially defies precise description. Even if it were available, the size of the associated computational problem would exceed our ability to solve it for any useful size of spatial domain. Consequently, "macroscopic" models are used to describe flow within permeable media. 
The macroscopic equations correspond to a continuum representation at a larger scale than that for the fluid. The continuum represents local averages over many "pores", as compared to the microscopic description, for which the continuum represents averages over many molecules. Many models, initially developed empirically, are now firmly established using local volume-averaging of the associated molecular-scale continuum equations, or other homogenization methods (Slattery 1967; Whitaker 1969). These models firmly establish the functional relationships relating fluid states and the means for predicting the response to various inputs, given the fluid and media properties. However, the media properties represent local effective empiricisms, and their determination remains an outstanding problem.

The flow of a single fluid phase through a rigid permeable media is the most basic physical situation of interest. In this case, the volume-averaged mass balance provides the equation of continuity:

$$
\frac{\partial(\phi \rho)}{\partial t}=-\nabla \cdot(\rho \mathbf{v})
$$

where $\mathbf{v}$ is the superficial velocity, $\rho$ the density, and $\phi$ the porosity. Volume averaging the differential momentum balance for the same physical situation yields Darcy's law:

$$
\mathbf{v}=-\frac{\mathbf{k}}{\mu} \cdot(\nabla p-\rho \mathbf{g})
$$

where $p$ is the pressure, $\mu$ the viscosity, $\mathbf{g}$ the acceleration of gravity, and $\mathbf{k}$ the permeability. The flow of a single fluid phase through permeability media can be simulated for any particular situation by solving Eqs. 1 and 2 with the pertinent boundary and initial conditions and fluid and media properties.

The velocity and pressure are system states which depend explicitly on position and time. Each represents an average over a volume element centered at the position at which they are specified. The porosity and permeability are properties of the media, similarly defined relative to a local volume element. A tenet of local volume-averaging is that it is not necessary to specify the actual size of the volume element, provided it is relatively large compared to a pore, but relatively small compared to the sample. The power of these equations is that the functional relationships among the properties and states are specified, and the detailed configuration of the solid surface is not required to describe the pressure and flow associated with a scale that is larger than the pore scale, yet is resolved spatially within the sample. However, the media properties are effective properties that must be determined. The finest resolution available for the properties, which we refer as the intrinsic scale, will be that corresponding to the minimum size of the averaging volume. This must 
necessarily include a number of "pores"-nominally, tens, or perhaps hundreds, of pores. While the minimum size for the averaging volume cannot really be stated precisely, it is clear that it must be much smaller that the typical size of a core sample. We believe that our measurements of porosity and permeability, which are resolved to the millimeter or sub-millimeter scale, are good representations of intrinsic properties.

To describe the flow of two immiscible fluid phases, we write a continuity and Darcy equation for each fluid phase:

$$
\begin{gathered}
\frac{\partial\left(\phi \rho_{i} s_{i}\right)}{\partial t}=-\nabla \cdot\left(\rho_{i} \mathbf{v}_{i}\right) \\
\mathbf{v}_{i}=-\frac{k k_{r i}}{\mu_{i}}\left(\nabla p_{i}-\rho_{i} \mathbf{g}\right)
\end{gathered}
$$

where $s_{i}$ is the saturation of phase $i$ and $k_{r i}$ is the relative permeability to phase $i$. The fluid saturations are related by:

$$
s_{1}+s_{2}=1
$$

Locally, pressures for the two fluid phases are related by the capillary pressure:

$$
p_{c}\left(s_{1}\right)=p_{1}-p_{2}
$$

where phase 1 is taken as the nonwetting phase.

The relative permeability and capillary pressure, to which we will refer collectively as multiphase flow functions, are properties of the media and fluids. They are primarily functions of fluid saturation. In principle, they could vary with position. However, it is presumed that the primary spatial variations are taken into account through the porosity and permeability, so that it is usual to assume the multiphase flow properties are uniform over a larger spatial region, perhaps as defined by distinct geological units. At any rate, we will take them to be uniform within any core sample.

\subsection{Determination of properties}

The porosity and permeability are effective properties which are required to model flow associated with any process within permeable media. In addition to these, two relative permeability functions and a capillary pressure function are required to simulate the flow of two immiscible phases.

The porosity, which is the void fraction of the media, can be determined as the ratio of the volume of saturating fluid to the total volume containing the fluid and solid. Traditionally, an average value for porosity is determined gravimetrical. Spatial distributions of porosity can be 
determined using non-invasive spatially resolved measurements within the sample. X-ray CT scanning (Withjack 1988) can be used to determine the porosity distribution. Here, we use MRI, which has the advantage that the entire three-dimensional distribution can be determined, rather than that associated with a selection of two-dimensional images. The quantitative resolution of the MRI signal to assess the amount of saturating fluid is a particular challenge addressed in this project.

The determination of the permeability is considerably more challenging. The permeability is in essence defined by the Darcy equation (see Eq. 2). That is, the permeability is the value that relates, at any position, the gradient of the pressure to the velocity. It must be determined by setting up an experiment, measuring fluid states, or functions of the states, and the solving an associated inverse problem based on a mathematical model of the experiment. This is demonstrated for the traditional method for determining the permeability of a core sample.

A fluid is flowed unidirectionally through a porous sample, and the flow rate into the sample and the pressure drop across the sample are measured. For a sample of length $L$ with a constant crosssectional area $A_{c}$, when body forces are assumed to be negligible, the permeability is calculated by

$$
k^{a p p}=-\mu \frac{Q}{A_{c}} \frac{L}{\Delta p}
$$

where $Q$ is the volumetric flow rate, $\Delta p$ is the pressure drop measured over the sample length $L$, and $k^{a p p}$ is the apparent permeability in the overall direction of flow. This equation follows from the general state equations (Eqs. 1 and 2) and represents a model for the particular experiment used. However, this incorporates the assumption that the permeability is uniform within the sample. If the material is not homogeneous, the measured value of permeability so obtained is not correct. Consequently, we refer to the value so calculated to as an "apparent" value-i.e., it is calculated on the basis of an equation that may not correctly describe the physical problem.

To illustrate the severity of error possible, consider the simple case for which the sample is solid, except for a single fracture which intersects two opposite faces of the sample. If flow injection is attempted into a face without the fracture, the permeability will be zero. Fluid will flow when injected into a face having the fracture, and a non-zero permeability can be calculated. The apparent permeability thus depends on the particular orientation of the sample, and neither case provides a good estimate for an "average" permeability.

In this work, we develop an approach that provides, for the first time, the spatial distribution of permeability within core samples. It is based on the use of MRI to measure velocity distributions, and the use of those measurements with an associated inverse problem to determine the permeability 
distribution.

The relative permeability is also defined through the Darcy expression (see Eq. 4), and must be determined through an associated inverse problem. It is essential that the mathematical models used in the inverse problems be an accurate representation of the physical phenomena occurring within the experiment. We have developed a general approach for determining relative permeability, together with capillary pressure, from displacement experiments (Watson, Kulkarni, Nordtvedt, Sylte, and Urkedal 1998). It utilizes a novel stepped-injection scheme and MRI observations of saturation to obtain accurate estimates of the functions (Kulkarni et al. 1998). For all applications, an apparent value of permeability has been used. However, if the actual intrinsic permeability were determined, it could be incorporated into the inverse problem to avoid errors in the determination of the properties that would result from inaccurate modeling of the associated experiment. In this work, we explore the effect of spatial variations of properties on the accuracy of estimates of the multiphase flow functions, and advance three-dimensional simulation as an element in determining the flow functions.

\subsection{Prediction of properties}

While flow experiments must be used to measure properties such as the absolute and relative permeability, it is most desirable to develop the means to predict permeability from direct measurements. This can be done through the development of empirical correlations that relate measured values of permeability and other properties, which we call predictors. The use of NMR well logging measurements to predict permeability has been a very active area of research. An example of such a prediction is provided by the following correlation developed by (Kenyon et al. 1988):

$$
k=a \phi^{4} T_{m}^{2}
$$

where $T_{m}$ is the mean NMR relaxation time. Normally, the coefficient $a$ is calculated on the basis of a calibration using a set of samples corresponding to a particular region within the reservoir for which measurements of apparent permeability, average porosity, and average relaxation have been determined for each sample.

With the intrinsic properties, we believe that much more reliable predictive equations can be developed since these properties provide thousands of data for each sample. Furthermore, such observations provide a better opportunity to identify intrinsic correlations among the properties that may be hidden by the use of average or apparent values for spatially variable properties. 


\section{Determination of NMR Relaxation Distributions}

A key NMR property is relaxation. The relaxation of fluids in permeable media is profoundly affected by the solid surfaces. In some cases this can be a nuisance. For example, the (intrinsic) magnetization intensity at equilibrium is proportional to the amount of fluid observed, and thus its estimation forms the basis for determination of the porosity, when the sample is fully saturated with the observed fluid, or saturation, when it is partially saturated. However, the intrinsic intensity can not be measured directly, and it must be determined under dynamic conditions. Unlike with bulk fluids, the relaxation of fluids in permeable media is generally so fast that appreciable relaxation occurs before the signal is acquired. In order to get accurate estimates of porosity and saturation, it is necessary to model the relaxation process (see Section 3.2) in order to estimate the intrinsic magnetization intensity.

On the other hand, relaxation can be a source of important information. Since relaxation depends on the actual structure of the permeable media, relaxation can provide information to estimate the pore-size distribution. In fact, NMR is probably the best method currently available to probe micro-structures. The critical information for either of these situations is the estimation of the relaxation distribution from NMR data. This is fundamentally an ill-posed estimation problem. While a number of methods have been proposed, it was necessary for us to develop a new approach in order to get reliable solutions to this problem. We describe that method in this section.

\subsection{Introduction}

The relaxation of NMR active nuclei, or spins, is a fundamental NMR property. In an NMR relaxation experiment, a substance containing NMR active nuclei is placed within the static magnetic field. The ensemble of spin magnetization is initially parallel to the axial direction of the static magnetic field. Suppose a $90^{\circ}$ radio-frequency (RF) pulse is applied, so that the spin magnetization flips down to the transverse plane, which is perpendicular to the axial direction. Then, the magnetization relaxes back to the equilibrium state. During the relaxation process, the projection of the magnetization on the transverse plane decreases to zero while that on the axial direction increases to the intrinsic magnetization of the equilibrium state. These two types of relaxation are called transverse and longitudinal relaxations, respectively.

In this work, we deal with permeable media samples saturated with fluids, such as water and oil, and we detect the signal from hydrogen nuclei in the fluids. The fluid in a permeable medium 
experiences various magnetic field environments depending on the pore structure where the fluid is located. In particular, the spins of fluid in the immediate vicinity of interfaces of fluid and solid undergo enhanced relaxation as compared to those under bulk conditions due to the interaction with the solid. In heterogeneous porous media, the relaxation rate is affected by a number of dissimilar magnetic susceptibilities and chemical components, and consequently the relaxation data reflect superposition of various relaxation rates.

The magnetization evolution of NMR relaxation is modeled in terms of characteristic relaxation times. If the spins in a sample relax with the same rates, the relaxation data is described by a single characteristic relaxation time. On the other hand, there is a number distribution of characteristic relaxation times-conventionally referred to as NMR relaxation distribution-for fluids in heterogeneous porous media. Once the relaxation distribution is known, the corresponding magnetization can be calculated with the model. However, the relaxation distribution cannot be directly calculated from the relaxation data. Instead, an inverse problem is posed and solved. Here, the relaxation distribution function is determined such that is most consistent with the data under the given conditions.

In this inverse problem, the mathematical representation of the distribution function is critical to obtain reliable solutions. Previously, relatively simple functions such as multi-modal deltafunctions, piecewise-constant models and interpolants of discrete points (Timur 1969; Kroeker and Henkelman 1986; Kenyon et al. 1989; Whittall and MacKay 1989; Whittall et al. 1991; Howard and Spinler 1993; Kleinberg 1996) were used to represent the distribution. However, these representations inherently have limitations arising from the specific shape of the functions. In this project, we have developed a nonparametric regression method to identify the NMR relaxation distribution function. The relaxation distribution function is represented by spline functions so that any continuous distribution function can be arbitrarily accurately represented. The determined NMR relaxation distribution can provide important structural information about heterogeneous materials such as pore-size distributions (see Section 4) and the means to recover quantitative spin-density information from MRI experiments (see Section 5).

It has been known that the nonparametric estimation of relaxation distributions is ill-conditioned, which means that small changes of errors in the input can seriously affect the solution. The use of regularization is one of the most appropriate ways to handle such problems (Wahba 1978). The regularization penalizes variations in the determined distribution insofar as the estimation does not compromise the consistency with data. The degree of regularization is controlled by a specified 
scalar, the regularization parameter. The methodology used to choose the value of the regularization parameter is critical. Graphical or other ad-hoc methods are often used to determine the regularization parameter (Hansen 1992; Liaw etal. 1996; Kulkarni and Watson 1997), but these require user intervention. While these methods may be suitable for analysis of a few data sets, their hands-on approach is prohibitive when analyzing a large number of data sets, as required with MRI. In this project, we have developed a data-driven technique based on statistical criteria to determine regularization parameter. The nonparametric approach together with the developed methodology for determining regularization parameter provide reliable estimation of the relaxation distributions. The detailed methodology and the validations are described in the following sections.

\subsection{Modeling NMR relaxation distributions}

The normalized relaxation distribution function, $P(\tau)$, presents the normalized number density of spins that relax with characteristic time $\tau$. The magnetization value $M(t)$ at a relaxation time $t$ is written in terms of $P(\tau)$ :

$$
M(t)=M_{0} \int_{0}^{\infty} P(\tau) K(t, \tau) d \tau,
$$

which is a Fredholm integral equation of the first kind. Here, $M_{0}$ is the intrinsic magnetization and $K(t, \tau)$ is the kernel corresponding to the relaxation experiment. The normalized distribution function, $P(\tau)$, satisfies

$$
\int_{0}^{\infty} P(\tau) d \tau=1
$$

The kernel function, $K(t, \tau)$, is formulated depending on the NMR experiment. It is $\exp (t / \tau)$ for the spin-echo experiment that measures transverse $\left(T_{2}\right)$ relaxation, and $1-\alpha \exp (t / \tau)$ for the inversion-recovery experiment that measures longitudinal $\left(T_{1}\right)$ relaxation. Here, $\alpha$ is a parameter that accounts for inhomogeneities of the RF pulse in an inversion-recovery experiment. Equation 9 can be rewritten as

$$
Y(t)=\int_{0}^{\infty} P(\tau) \exp \left(\frac{t}{\tau}\right) d \tau
$$

for either type of relaxation experiment by representing the normalized magnetization, $Y(t)$, as $M(t) / M_{0}$ for $T_{2}$ relaxation and as $\left[M_{0}-M(t)\right] /\left(\alpha M_{0}\right)$ for $T_{1}$ relaxation.

The relaxation distribution, $P(\tau)$, is a physical property that is expected to be well represented by a smooth, continuous function. Therefore, it is appropriate to represent the function in terms of splines. Splines are piecewise polynomials whose different polynomial segments are joined together at knots in a way that ensures continuity properties. It is known that every continuous function 
on a finite domain can be approximated arbitrary accurately by splines with sufficient number of knots (Schumaker 1981). The relaxation distribution function can be represented in terms of B-spine functions as

$$
P(\tau)=\sum_{i=1}^{n_{s}} c_{i} B_{i}^{m}(\tau, \mathbf{x})
$$

Here, $B_{i}^{m}(\tau, \mathbf{x})$ are B-spline basis functions where $m$ is the order of the spline and $\mathbf{x}$ represents the extended partition-the location of the knots. Once the partition is chosen, the distribution is specified by the complete set of coefficients, $c_{i}$. The number of coefficients is given by the degrees of freedom $n_{s}$, which is the sum of the order and the number of interior knots. In our work, a sufficiently large dimension of the spline space $n_{s}$ is used so that the estimation of the distribution function is not significantly influenced by the number and position of knots.

To estimate $P(\tau)$, a performance index is formulated and subsequently minimized:

$$
J=\left[\mathbf{Y}^{\text {data }}-\mathbf{Y}^{c a l c}\right]^{T} \mathbf{W}\left[\mathbf{Y}^{\text {data }}-\mathbf{Y}^{c a l c}\right]+n \lambda \int_{0}^{\infty}\left[\frac{d^{2} P(\tau)}{d \tau^{2}}\right]^{2} d \tau .
$$

The first term in the performance index reflects the precision of fit of the data vector $\mathbf{Y}^{\text {data }}$, comprised of the data $Y^{\text {data }}\left(t_{j}\right), j=1, \ldots, n$, to the corresponding calculated values, $\mathbf{Y}^{\text {calc }}$, evaluated by Eq. 11. The weighting matrix $\mathbf{W}$ is chosen on the basis of maximum likelihood principles (Beck and Arnold 1977). In our work, we assume that the errors have mean zero and are identically distributed, so that $\mathbf{W}$ is identity matrix.

The second term in Eq. 13 is the regularization term. It is used to stabilize the solution by enforcing certain constraints on the determined distribution or its derivatives. In the present case, the regularization term takes the form of the second derivative operator which acts to penalize a lack of smoothness and sharp changes in the estimated distribution function. The regularization term is weighted relative to the data fitting term by a scalar, $\lambda$, the regularization parameter. It controls the trade-off between the smoothness of the solution and the goodness of fit to the data in the performance index. Large values tend to give biased estimates that and over-smooth the distribution function, leading to an imprecise fit to the data. Small values often provide unrealistic solutions with high variability.

The calculated values of the observed data are written in terms of the B-spline coefficients, $\mathbf{c}$, by

$$
\mathbf{Y}^{\text {calc }}=\mathbf{A c}
$$

where the components of $\mathbf{A}$ are determined by evaluating the kernel function in Eq. 9 with the 
spline basis functions in Eq. 12:

$$
a_{i, j}=\int_{\tau_{\min }}^{\tau_{\max }} B_{i}^{m}(\tau, \mathbf{x}) \exp \left(\frac{t_{j}}{\tau}\right) d \tau
$$

Here, $\tau_{\min }$ and $\tau_{\max }$ represent the minimum and maximum boundaries of the distribution function, respectively. The distribution function is zero outside of these boundaries. The performance index in Eq. 13 is then written formally as a quadratic least squares minimization problem:

$$
\min _{\mathbf{c}} J=\left\|\mathbf{Y}^{\text {data }}-\mathbf{A c}\right\|^{2}+n \lambda\|\mathbf{M c}\|^{2}
$$

The quantity $\mathbf{M}^{T} \mathbf{M}$ is formed by evaluating integrals of the products of the second derivatives of the B-spline basis functions. Linear equality constraints,

$$
\mathbf{G c}=\mathbf{0},
$$

and inequality constraints,

$$
\Gamma \mathbf{c} \geq \mathbf{0},
$$

are included to further stabilize the solution. Inequality constraints ensure that estimated distribution is non-negative, while equality constraints are used to enforce the desired derivatives of the determined distribution at the boundaries.

For a specified value of the regularization parameter, $\lambda$, and a given set of relaxation data, $\mathbf{Y}^{\text {data, }}$ there is a unique and global solution that minimizes Eq. 16 with the given equality and inequality constraints. The expression of the solution can be obtained by transforming the constrained problem to an unconstrained problem. Here, the active inequality constraints are identified and treated as equality constraints, i.e., they are added to the equality constraints and any dependent constraints are eliminated. Then, the minimization problem with equality constraints is transformed to an unconstrained problem (see Appendix 1 for the derivation). The performance index with inequality and equality constraints (Eqs. 16-18) is equivalently written as

$$
\min _{\mathbf{c}} J=\left\|\mathbf{Y}^{\text {data }}-\tilde{\mathbf{A}} \mathbf{c}\right\|^{2}+n \lambda\|\tilde{\mathbf{M}} \mathbf{c}\|^{2} .
$$

The matrices $\mathbf{A}$ and $\mathbf{M}$ of the constrained problem are now replaced by $\tilde{\mathbf{A}}$ and $\tilde{\mathbf{M}}$ of the unconstrained problem. The matrices $\tilde{\mathbf{A}}$ and $\tilde{\mathbf{M}}$ are defined in Appendix 1 and they essentially incorporate the inequality and equality constraints.

The optimal B-spline coefficients $\hat{\mathbf{c}}_{\lambda}$ for a given $\lambda$ can be expressed as

$$
\hat{\mathbf{c}}_{\lambda}=\left[\tilde{\mathbf{A}}^{T} \tilde{\mathbf{A}}+n \lambda \tilde{\mathbf{M}}^{T} \tilde{\mathbf{M}}\right]^{-1} \tilde{\mathbf{A}}^{T} \mathbf{Y}^{d a t a}
$$


Although $\hat{\mathbf{c}}_{\lambda}$ is provided explicitly by Eq. 20, it is not calculated in that way due to numerical issues associated with the formation of a matrix inverse. We use a numerical least squares solver (Haskell and Hanson 1981) to obtain the optimal coefficients, $\hat{\mathbf{c}}_{\lambda}$. We also exploit this solver to identify the active inequality constraints. The relaxation distribution function corresponding to the optimal B-spline coefficients is calculated by

$$
\hat{P}_{\lambda}(\tau)=\sum_{i=1}^{n_{s}} \hat{c}_{\lambda i} B_{i}^{m}(\tau, \mathbf{x}),
$$

and the corresponding normalized magnetization is

$$
\hat{\mathbf{Y}}_{\lambda}=\mathbf{A} \hat{\mathbf{c}}_{\lambda}
$$

Combining Eqs. 20 and 22, the predicted values are expressed as

$$
\begin{aligned}
\hat{\mathbf{Y}}_{\lambda} & =\tilde{\mathbf{A}}\left[\tilde{\mathbf{A}}^{T} \tilde{\mathbf{A}}+n \lambda \tilde{\mathbf{M}}^{T} \tilde{\mathbf{M}}\right]^{-1} \tilde{\mathbf{A}}^{T} \mathbf{Y}^{\text {data }} \\
& =\mathbf{H}(\lambda) \mathbf{Y}^{\text {data }}
\end{aligned}
$$

where $\mathbf{H}(\lambda)$ is the hat matrix.

The statistical criteria for determining the optimal regularization parameter are developed in terms of the hat matrix (see Section 3.3). Here, the hat matrix is treated as a function of $\lambda$ for

given matrices of $\tilde{\mathbf{A}}$ and $\tilde{\mathbf{M}}$ which are not dependent on $\lambda$. However, a problem arises in calculating the hat matrix because the active inequality constraints change with respect to $\lambda$, and consequently $\tilde{\mathbf{A}}$ and $\tilde{\mathbf{M}}$ are dependent on $\lambda$. We use an iteration method suggested by Villalobos and Wahba (1987) to solve this problem. Here, $\tilde{\mathbf{A}}$ and $\tilde{\mathbf{M}}$ are first determined without inequality constraints. The optimum $\lambda$ with those matrices are determined and, under this $\lambda$, new $\tilde{\mathbf{A}}$ and $\tilde{\mathbf{M}}$ are found. Then, a new optimal $\lambda$ is determined with the new $\tilde{\mathbf{A}}$ and $\tilde{\mathbf{M}}$. This procedure is repeated until $\lambda$ converges to the optimal value.

\subsection{Statistical selection of regularization parameter}

Nonparametric statistical theory provides the framework for developing a reliable data-driven method to select the regularization parameter (Eubank 1988; Eubank 1999). Here, an additive error model is used to represent the data, $y_{j}$, observed at time $t_{j}$ :

$$
y_{j}=Y\left(t_{j}, P\right)+\epsilon_{j}, \quad j=1, \ldots, n,
$$

where $P$ represents the true but unknown distribution function and $\epsilon_{j}$ is a random measurement error with zero mean and variance $\sigma^{2}$. The normalized magnetization, $Y$, defined in Eq. 11 is now 
expressed as a function of the distribution function, $P$. The criteria to provide assessments of an estimator's performance can be expressed in terms of $Y\left(t_{j}, P\right)$. Examples are the loss:

$$
L(\lambda)=\frac{1}{n} \sum_{j=1}^{n}\left[Y\left(t_{j}, P\right)-Y\left(t_{j}, \hat{P}_{\lambda}\right)\right]^{2},
$$

the risk, which is the expected value of the loss:

$$
R(\lambda)=\mathrm{E}[L(\lambda)]
$$

and the prediction risk:

$$
R_{p}(\lambda)=\mathrm{E}\left[\frac{1}{m} \sum_{i=1}^{m}\left(y_{i}^{\star}-Y\left(t_{i}, \hat{P}_{\lambda}\right)\right)^{2}\right] .
$$

The distribution function $\hat{P}_{\lambda}$ is determined by the $n$ data values $\left(y_{1}, \ldots, y_{n}\right)$ for a given value of $\lambda$. The prediction risk is based on $m$ observations, $y_{i}^{\star}=Y\left(t_{i}, P\right)+\epsilon_{i}^{\star}(i=1, \ldots, m)$, which are independent from the $n$ measurements used for $\hat{P}_{\lambda}$. The prediction risk is related to the risk by

$$
R_{p}(\lambda)=\sigma^{2}+R(\lambda)
$$

The value of $\lambda$ that minimizes either the loss, risk, or prediction risk can be the optimal value. However, these criteria are defined in terms of the unknown distribution function, $P$. We use $\hat{P}_{\lambda}$ as an estimator of $P$ so that these criteria can be developed in terms of the measured data.

The estimator $\hat{P}_{\lambda}$ provides the mean squared error (MSE),

$$
\operatorname{MSE}(\lambda)=\frac{1}{n} \sum_{j=1}^{n}\left[y_{j}-Y\left(t_{j}, \hat{P}_{\lambda}\right)\right]^{2},
$$

which gives a biased estimate of prediction risk because

$$
\mathrm{E}[\mathrm{MSE}]-R_{p} \neq 0
$$

Knowledge of the bias value, calculated to be $2 n^{-1} \sigma^{2} \operatorname{tr}[\mathbf{H}(\lambda)]$ (Eubank 1999), allows the unbiased prediction risk (UBPR) to be formed:

$$
\operatorname{UBPR}(\lambda)=\operatorname{MSE}(\lambda)+\frac{2}{n} \sigma^{2} \operatorname{tr}[\mathbf{H}(\lambda)]
$$

The relationship between the risk and prediction risk (Eq. 27) yields an expression for an unbiased estimator of the risk:

$$
\operatorname{UBR}(\lambda)=\operatorname{MSE}(\lambda)+\frac{2}{n} \sigma^{2} \operatorname{tr}[\mathbf{H}(\lambda)]-\sigma^{2} .
$$

In order to use UBR criterion, the variance, $\sigma^{2}$, must to be estimated. In this work, a nonparametric estimate of the variance introduced by Gasser et al. (1986) is used to calculate $\sigma^{2}$. 
Cross validation (CV) (Stone 1974), or PRESS (Allen 1974), is another criterion that estimates prediction risk. The $\mathrm{CV}$ criterion is given by

$$
\mathrm{CV}(\lambda)=\frac{1}{n} \sum_{j=1}^{n}\left[y_{j}-Y\left(t, \hat{P}_{\lambda}^{(j)}\right)\right]^{2} .
$$

The CV criterion differs from MSE (Eq. 29) in that the distribution function, $\hat{P}_{\lambda}^{(j)}$, is determined by $n-1$ data $\left(y_{1}, \ldots, y_{j-1}, y_{j+1}, \ldots y_{n}\right)$ in which the $j$ th datum, $y_{j}$, is omitted. This criterion is formed as such that each missing point $y_{j}$ is predicted as a measure of the goodness of $\lambda$. It can be shown that Eq. 33 is equivalent to (Craven and Wahba 1979)

$$
\mathrm{CV}(\lambda)=\frac{1}{n} \sum_{j=1}^{n}\left[\frac{y_{j}-Y\left(t_{j}, \hat{P}_{\lambda}\right)}{1-h_{j j}(\lambda)}\right]^{2},
$$

where $h_{j j}(\lambda)$ is the $j$ th diagonal element of the hat matrix, $\mathbf{H}(\lambda)$.

Generalized cross-validation (GCV) (Craven and Wahba 1979) is a modification of CV criterion. GCV criterion is given by

$$
\begin{aligned}
\operatorname{GCV}(\lambda) & =\frac{1}{n} \sum_{j=1}^{n}\left[\frac{y_{j}-Y\left(t_{j}, \hat{P}_{\lambda}\right)}{1-n^{-1} \operatorname{tr} \mathbf{H}(\lambda)}\right]^{2} \\
& =\frac{\operatorname{MSE}(\lambda)}{\left[1-n^{-1} \operatorname{tr} \mathbf{H}(\lambda)\right]^{2}}
\end{aligned}
$$

The GCV differs from CV in that the each residual is divided by the average of the denominator, $1-h_{j j}(\lambda)$, which gives a more stable and faster evaluation of the criterion value.

\subsection{Validation with simulated data}

The developed methodology for determination of relaxation distribution is validated with simulated data. The simulated data were generated by simulating the NMR relaxation for a given true relaxation distribution (Fig. 1). In Fig. 1 and the following, the relaxation distribution, $P(\tau)$, is presented in a semi-log scale such that a portion of the area under the curve is proportional to the number of spins in the corresponding range of characteristic relaxation time.

We choose the kernel, $K\left(t_{j}, \tau\right)$, to be $\exp \left(-t_{j} / \tau\right)$. This kernel applies to conventional transverse or longitudinal relaxation experiments. Then, the simulated relaxation data, $y_{j}$, is calculated from the true relaxation distribution using Eq. 24. The measurement error, $\epsilon_{j}$, was generated by a random variable, $\epsilon$, following a normal distribution in which the $95 \%$ confidence interval is $|\epsilon| \leq \nu y_{\max }$. Here, $y_{\max }=\max \left[y\left(t_{j}, P\right)\right]=1$ and the noise, $\nu$, was chosen to be $0.1 \%, 1.0 \%$ and $5.0 \%$, respectively. The generated simulated data are presented in Fig. 2 for the three noise 
levels. We used cubic B-splines (the order of spline, $m$, is 4 ) for the representation of the relaxation distribution. We selected the number of knots to be 300 . They are logarithmically spaced between $\tau_{\min }=10^{-6} \mathrm{~s}$ and $\tau_{\max }=10 \mathrm{~s}$. The validation for the selection of the number of knots is provided in this section.

The relaxation distributions were estimated for the three data sets. The regularization parameter was determined using the UBR, CV, and GCV criteria. As described in Section 3.2, the optimal regularization parameter was determined by an iteration method. Figure 3 presents an example of how this works. The figure shows the CV scores calculated for the $5.0 \%$ noise data. Figure 3(a) is zoomed in 3(b) to clearly show the region containing the minimum. The CV scores for the first score line are calculated without inequality constraints and the first optimal $\lambda$ that minimizes the scores is determined. Then, the active constraints are identified with the first optimal $\lambda$. While these active constraints are fixed, the scores for the second score line are calculated and the second optimal $\lambda$ that minimizes the second scores is determined. This procedure is repeated until the change of the optimal $\lambda$ is sufficiently small $\left(<1.0 \times 10^{-25}\right.$ in this work $)$. As shown in the figure, the optimal $\lambda$ quickly converged for all of the analyzed simulated data, i.e., the desired order of accuracy was achieved at the first few iteration steps.

The determined regularization parameters are summarized in Table 1 with their corresponding scores. The regularization parameter that minimizes the loss (Eq. 26), $\lambda_{\text {loss }}$, is also presented in the table as well as the corresponding value of loss, $L\left(\lambda_{\text {loss }}\right)$, to be compared with the determined $\lambda$. Note that the loss can be calculated for each set of simulated data since the true relaxation distribution is known. The table shows that the determined $\lambda$ is close to $\lambda_{l o s s}$, which validates that the appropriate $\lambda$ is chosen by the statistical criteria. The regularization parameter value increases as the noise increases. This indicates that the smoothness of the solutions is more enforced at the cost of goodness of fit for the data as the data have bigger measurement errors.

It is important to use a sufficiently large number of knots in the relaxation distribution function, $P(\tau)$, which is represented by B-splines, as mentioned in Section 3.2. In this work, the number of knots is selected by observing $P(\tau)$ with respect to the number of knots: we increase the number of knots until there is no significant improvements in $P(\tau)$. In order to quantify the differences of a pair of distribution functions, we denote the $P(\tau)$ estimated using $N_{i}$ number of knots as $P_{N_{i}}(\tau)$ and define the integral-square-difference (ISD) of $P_{N_{i}}(\tau)$ and $P_{N_{j}}(\tau)$ as

$$
\operatorname{ISD}\left[P_{N_{i}}, P_{N_{j}}\right]=\int_{0}^{\infty}\left[P_{N_{i}}(\tau)-P_{N_{j}}(\tau)\right]^{2} d \tau
$$


We examined ISD for a series of number of knots such as $N_{i}=50,100,150,200,250,300,350$, 400 as shown in Fig. 4. The ISD values of adjacent $N_{i}$ are presented in Fig. 4(a). This graph shows that there are no significant improvements in $P(\tau)$ with respect to the one of adjacently smaller $N_{i}$ when $N_{i}$ is greater than 200. The ISD with respect to 400 knots (Fig. 4(b)) shows that there are no significant differences among the estimations with $N_{i}$ greater than 200 while the other three estimations with $N_{i}=50,100,150$, significantly deviate from them. We performed the similar analysis for other sets of data and chose to use 300 as the number of knots for all of them. The estimated relaxation distributions for each data set are presented in Fig. 5. The estimations are satisfactorily consistent with the true distribution, although there are deviations that become significant as the measurement error increases. The three criteria provide all similar estimations. In all estimations, the three major relaxation time ranges are identified. The predicted relaxation curves, calculated using the determined relaxation distribution, $y(t, P)=\int_{\tau_{\min }}^{\tau_{\max }} P(\tau) \exp (-t / \tau) d \tau$, are presented in Fig. 6 with the corresponding simulated relaxation data. The figure shows that the predictions are consistent with the data regardless of the noise levels. The three statistical criteria give virtually the same predicted relaxation curves. 


\begin{tabular}{|c|c|c|c|c|c|}
\hline noise & $\lambda_{\text {loss }}$ & $L\left(\lambda_{\text {loss }}\right)$ & criterion & $\lambda$, determined & score \\
\hline \hline $0.1 \%$ & $6.10 \times 10^{-18}$ & $2.44 \times 10^{-8}$ & $\mathrm{UBR}$ & $1.78 \times 10^{-18}$ & $3.30 \times 10^{-7}$ \\
& & & $\mathrm{CV}$ & $1.49 \times 10^{-19}$ & $2.52 \times 10^{-7}$ \\
& & & $\mathrm{GCV}$ & $9.10 \times 10^{-19}$ & $2.55 \times 10^{-7}$ \\
\hline $1.0 \%$ & $8.79 \times 10^{-18}$ & $2.22 \times 10^{-6}$ & $\mathrm{UBR}$ & $7.27 \times 10^{-17}$ & $2.72 \times 10^{-5}$ \\
& & & $\mathrm{CV}$ & $5.77 \times 10^{-17}$ & $2.70 \times 10^{-5}$ \\
& & & $\mathrm{GCV}$ & $5.88 \times 10^{-17}$ & $2.65 \times 10^{-5}$ \\
\hline \multirow{2}{*}{$5.0 \%$} & $4.33 \times 10^{-15}$ & \multirow{2}{*}{$3.84 \times 10^{-5}$} & $\mathrm{UBR}$ & $3.32 \times 10^{-15}$ & $6.41 \times 10^{-4}$ \\
& & & $\mathrm{CV}$ & $2.83 \times 10^{-15}$ & $6.37 \times 10^{-4}$ \\
& & & $\mathrm{GCV}$ & $3.06 \times 10^{-15}$ & $6.41 \times 10^{-4}$ \\
\hline \hline
\end{tabular}

Table 1: The results of the estimation using 300 B-spline knots

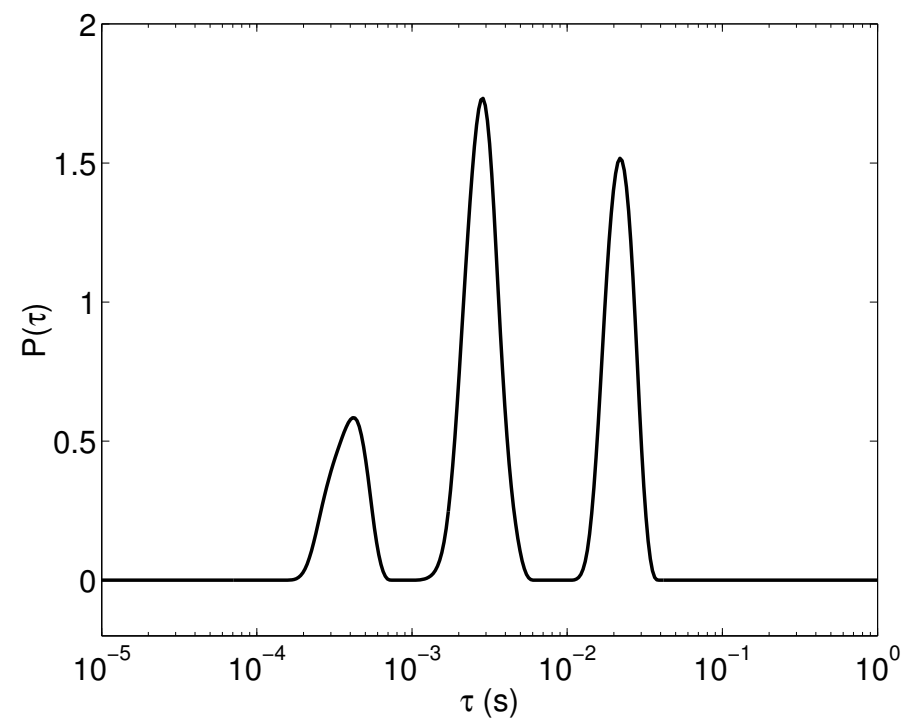

Figure 1: "True" relaxation distribution 


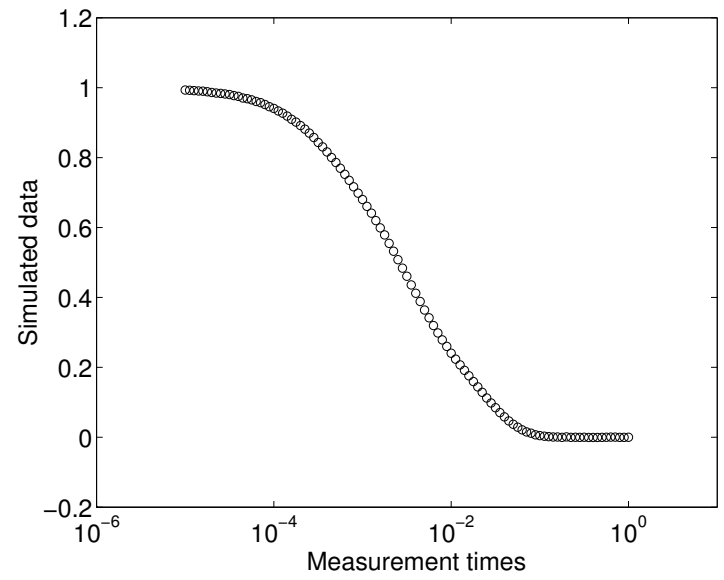

(a)

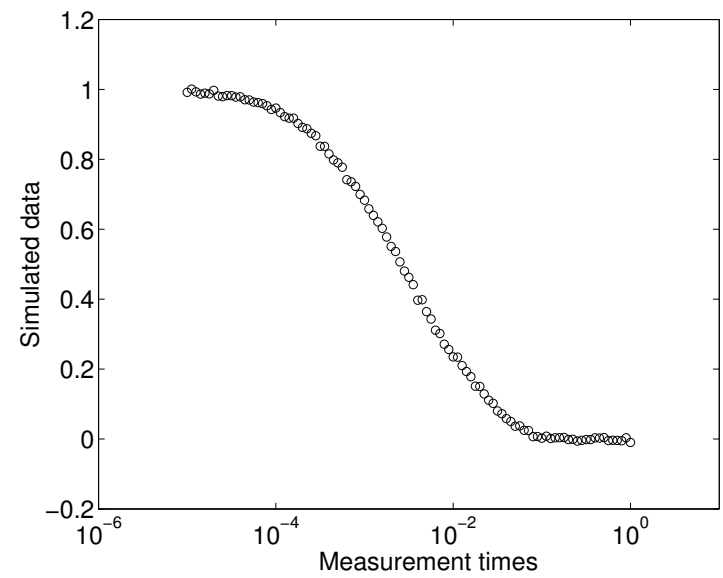

(b)

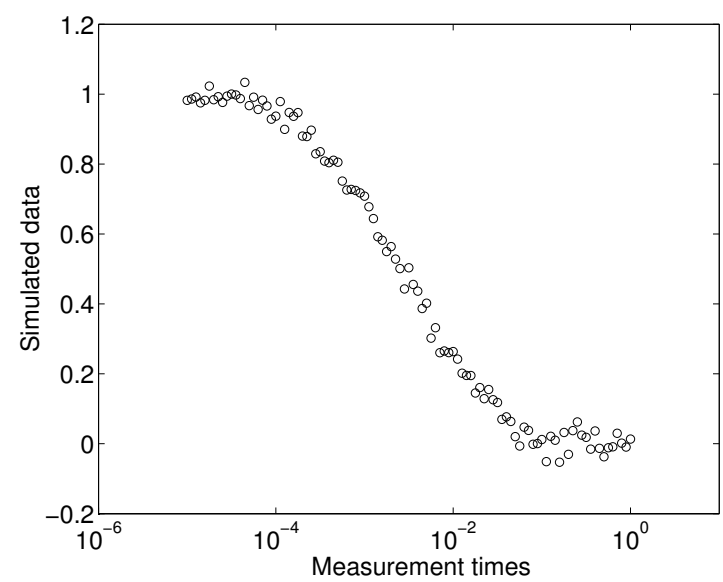

(c)

Figure 2: Simulated data. (a) $0.1 \%$ noise, (b) $1.0 \%$ noise, (c) $5.0 \%$ noise 


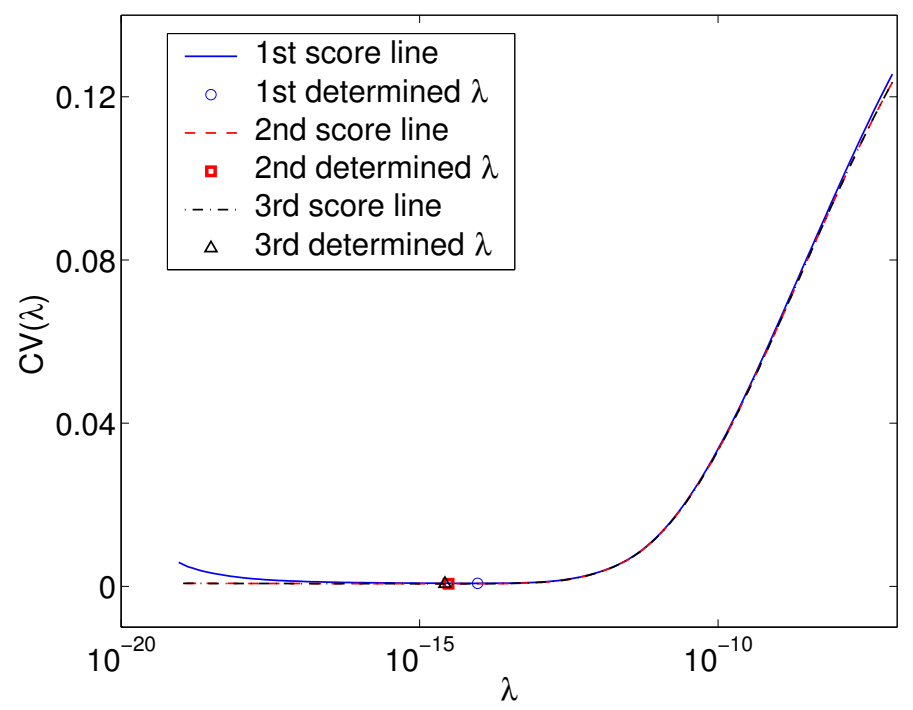

(a)

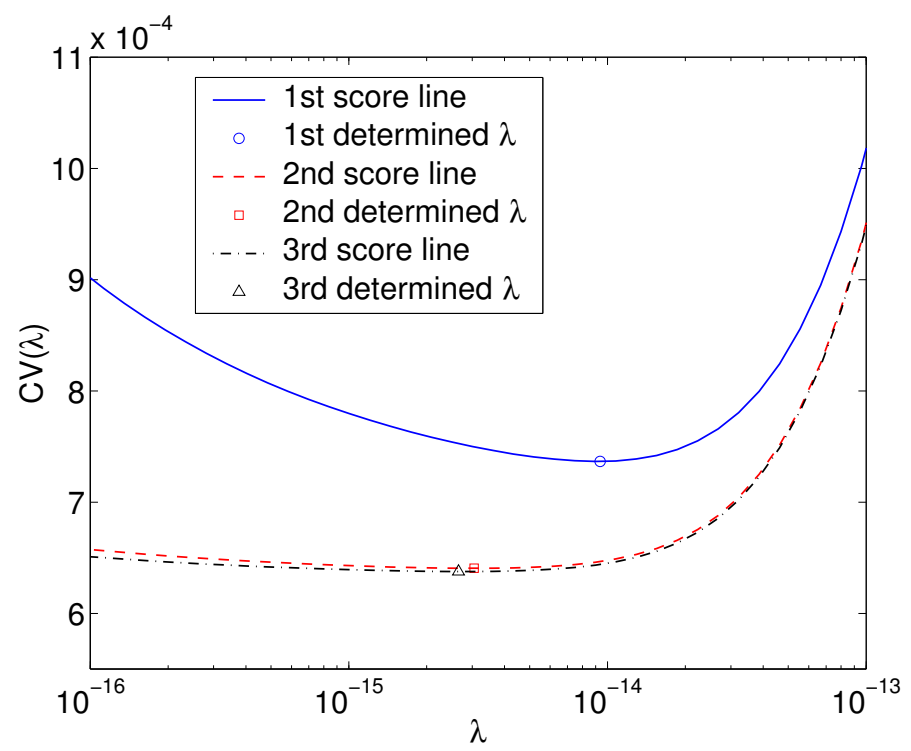

(b)

Figure 3: CV scores used for determination of the optimal $\lambda$. The $5.0 \%$ noise data are used. Figure (a) is zoomed in figure (b) with smaller axes scales 


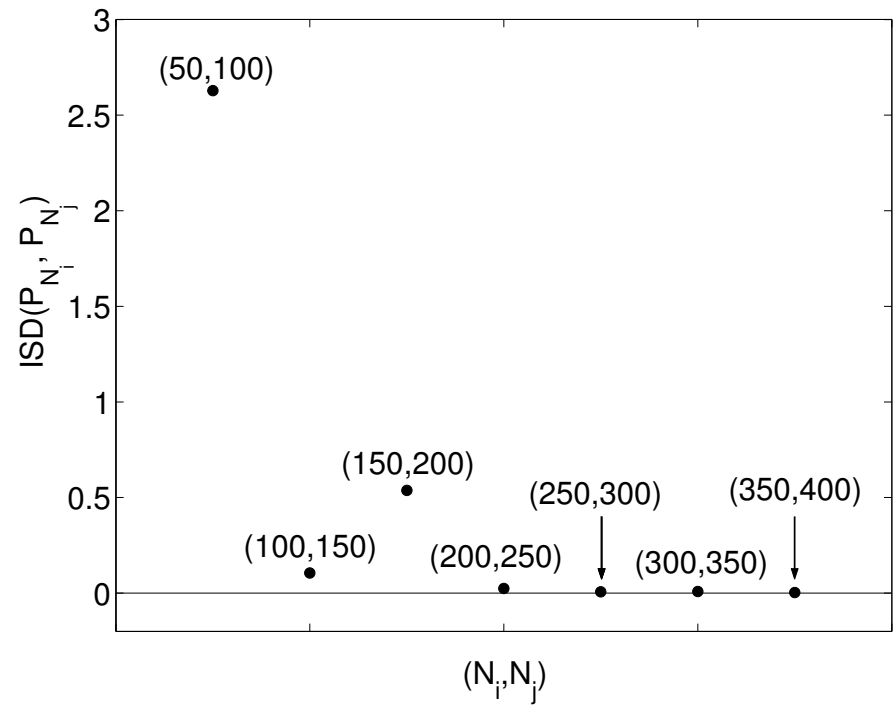

(a)

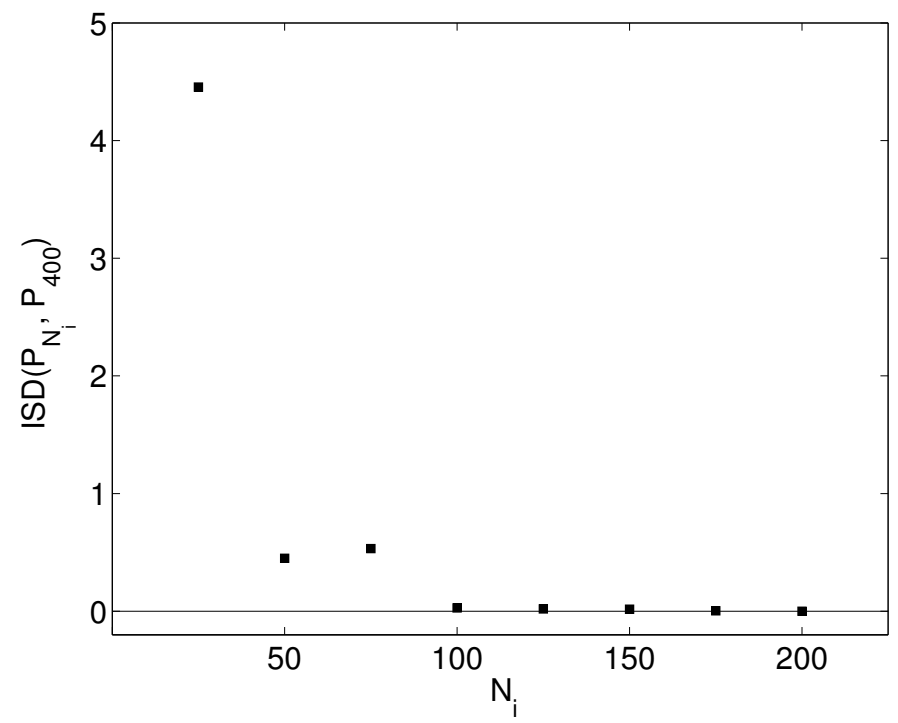

(b)

Figure 4: The integral-square-difference (ISD) of $P_{N_{i}}(\tau)$ and $P_{N_{j}}(\tau)$ for $1.0 \%$ noise data using UBR criterion (a) ISD for the adjacent numbers of knots among those examined (b) ISD with respect to the largest number of knots 


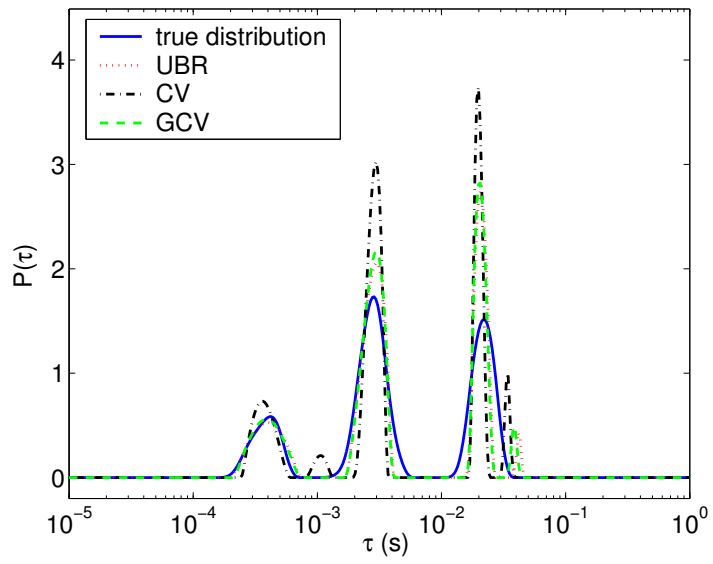

(a)

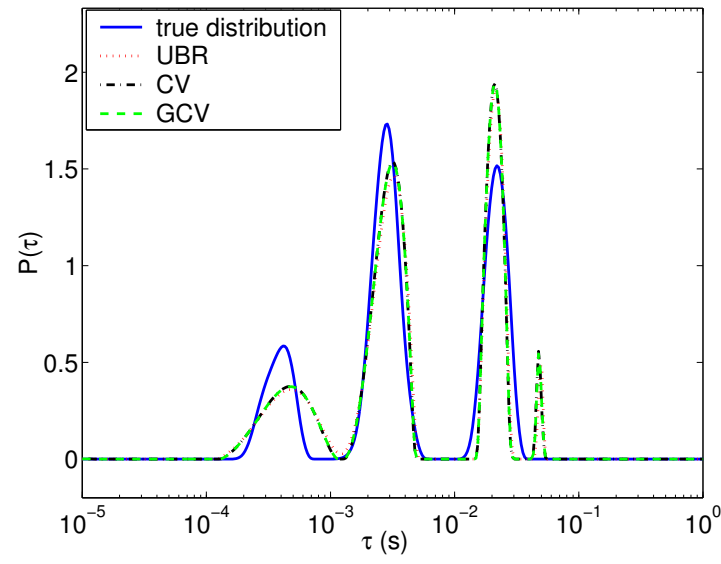

(b)

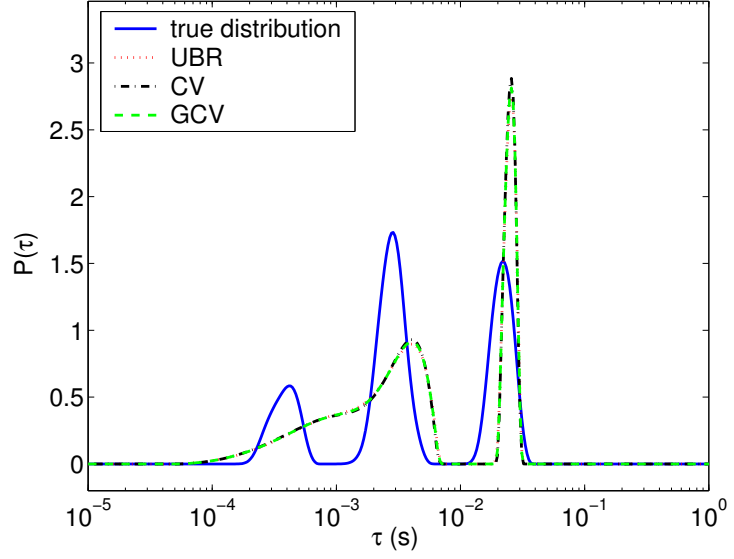

(c)

Figure 5: Estimated relaxation time distribution (a) $0.1 \%$ noise, (b) $1.0 \%$ noise, (c) $5.0 \%$ noise 


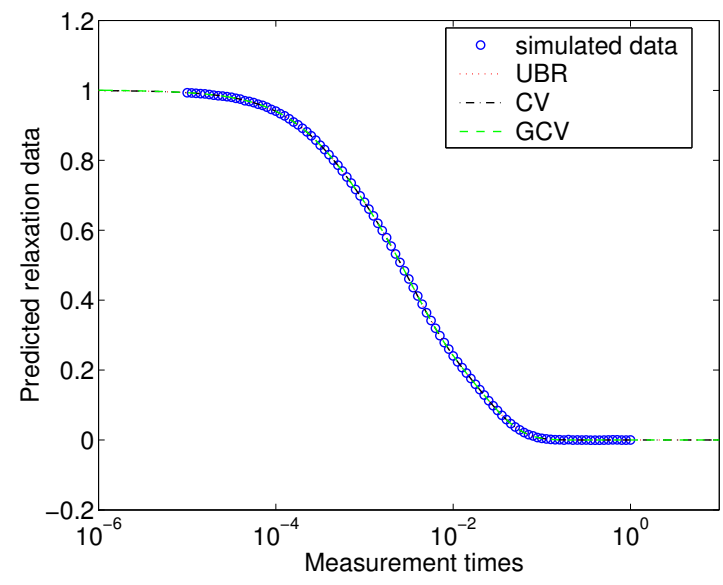

(a)

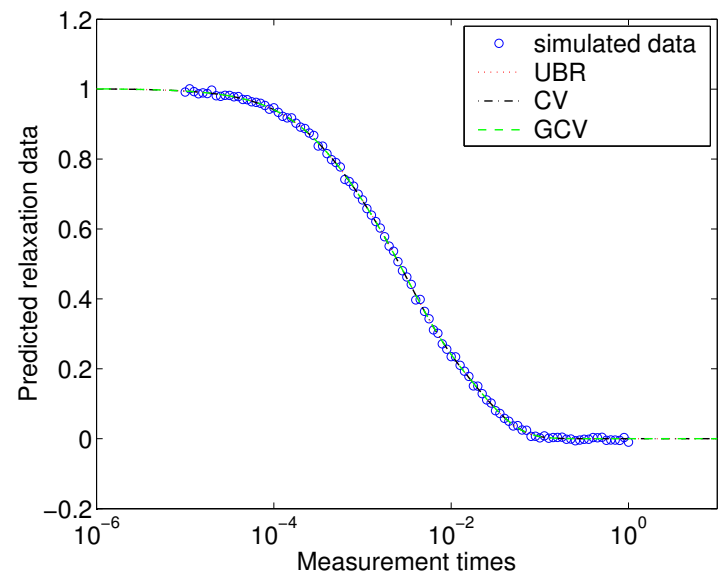

(b)

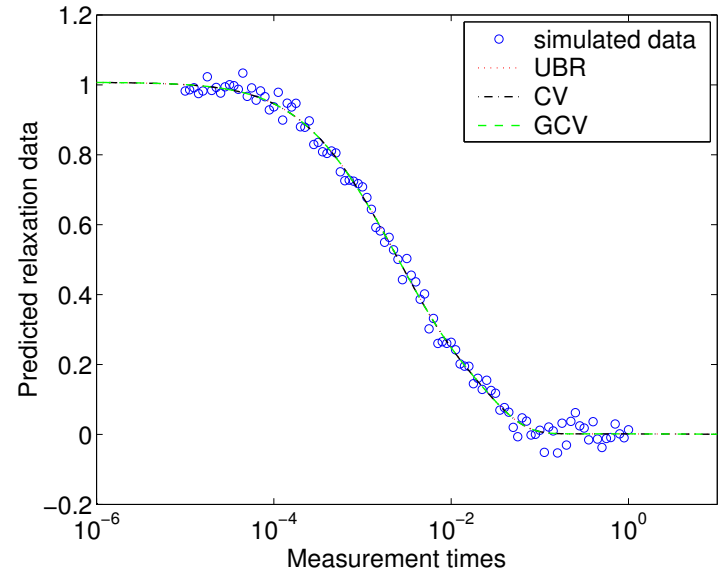

(c)

Figure 6: Predicted relaxation curves compared with the simulated data (a) $0.1 \%$ noise, (b) $1.0 \%$ noise, (c) $5.0 \%$ noise 


\begin{tabular}{||c||c|c|c|c|c|c||}
\hline \hline Sample & R138 & R145 & R207 & R221 & R237 & BENT1 \\
\hline \hline Diameter $(\mathrm{cm})$ & 2.5 & 2.5 & 2.5 & 2.5 & 2.5 & 2.5 \\
\hline Length $(\mathrm{cm})$ & 4.22 & 4.32 & 4.58 & 3.77 & 4.19 & 2.0 \\
\hline Fluid volume $\left(\mathrm{cm}^{3}\right)$ & 4.96 & 4.82 & 4.58 & 3.77 & 4.38 & 2.32 \\
\hline Bulk porosity & 0.240 & 0.227 & 0.223 & 0.189 & 0.213 & 0.237 \\
\hline
\end{tabular}

Table 2: The dimensions of the samples

\subsection{Analysis with experimental data}

We performed NMR relaxation experiments on several sandstone samples to determine the relaxation time distributions. Throughout this report, we demonstrate the experimental results for one Bentheimer sandstone sample (BENT1) and several actual reservoir sandstone samples (R138, R145, R207, R221, R237), and a thin Bentheimer sample (BENT2) used for velocity imaging in Section 6 . The reservoir samples were provided by a major petroleum company.

The reservoir sample and BENT1 are cylindrically shaped with dimensions given in Table 2 . The samples were sealed using epoxy (STYCAST ${ }^{\circledR}$ 2651) and surrounded by plexiglass for mounting in the RF probe. The hydrogen nuclei in the epoxy and the plexiglass have very small $T_{2}$ values so that they do not contribute to the observed signal intensity. The samples were vacuum saturated with brine solution (3 wt.\% NaCl, 0.03 wt. $\% \mathrm{NaN}_{3}$ in de-ionized water). The bulk porosity is calculated from the total volume of the fully saturated fluid in each sample divided by sample volume.

NMR experiments were performed with the Bruker BioSpec ${ }^{\circledR}{ }_{24} / 30$ system operated at $100 \mathrm{MHz}$. The system is equipped with \pm 20 Gauss $/ \mathrm{cm}$ gradient coil inside the $30 \mathrm{~cm}$ magnet bore. A 3.5cm-diameter birdcage coil was used in the experiments. All the experiments were performed at regulated room temperature $\left(21^{\circ} \mathrm{C}\right)$.

We performed inversion recovery experiment using $180^{\circ}$-delay- $90^{\circ} \mathrm{RF}$ pulse sequence to estimate $T_{1}$ relaxation distributions. The delay time between the two RF pulses corresponds to the measurement time. The delay times were carefully selected so that they cover the appropriate range of the $T_{1}$ relaxation times. The measured inversion-recovery data of BENT1 is presented in Fig. 7.

We use a Carr-Purcell-Meiboom-Gill (CPMG) (Carr and Purcell 1954; Meiboom and Gill 1959) sequence to measure the spin echo attenuation caused by $T_{2}$ relaxation and estimate the relaxation distribution. The pulse sequence is based on the spin-warp three-dimensional volume imaging 


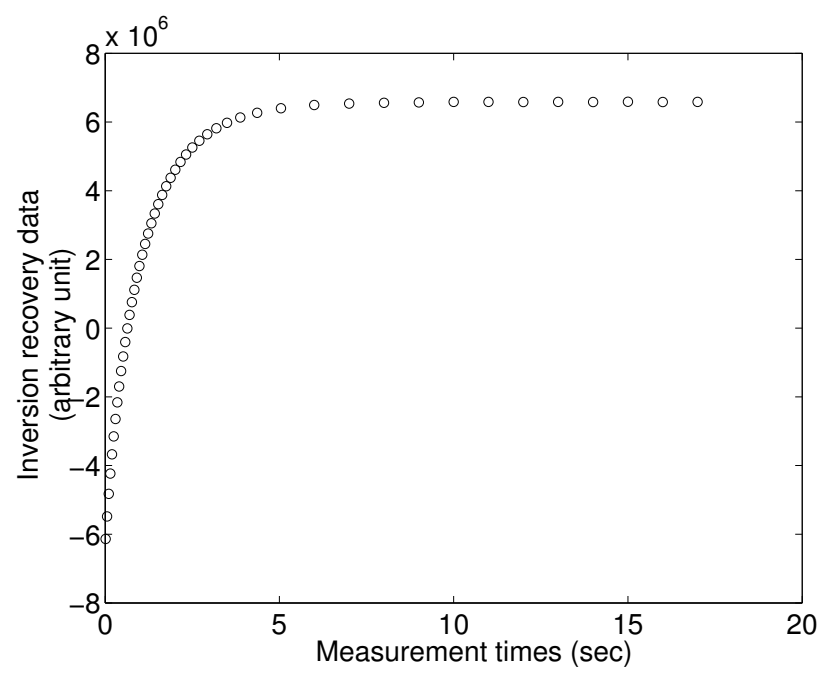

Figure 7: $T_{1}$ relaxation data by inversion-recovery experiment

sequence except that, following the initial excitation by a $90^{\circ}$ pulse, $180^{\circ}$ pulses continue to be applied with a spacing of $T E$ so that a train of echoes occurs. Both the first $90^{\circ}$ and subsequent $180^{\circ}$ pulses are nonselective broadband pulses. An obvious advantage of CPMG over the Hahn spin-echo is the shorter imaging time. An entire series of relaxation-attenuated CPMG data are collected in about the same time as a single spin-echo sequence. Another advantage is that the CPMG technique allows for the acquisition of $T_{2}$ data with the molecular diffusion time controlled by the experimentally selected value of echo spacing. The use of a short echo-spacing can minimize the diffusion attenuation rate and improve the interpretation of fitting parameters with suitable relaxation models.

The estimated $T_{1}$ and $T_{2}$ relaxation distributions for BENT1 are presented in Fig. 8. The relaxation distributions for the reservoir samples are provided in Appendix 2. We determined the harmonic average relaxation time, defined by

$$
\tau^{a v g}=\left[\int_{0}^{\infty} \frac{1}{\tau} P(\tau) d \tau\right]^{-1}, \quad \tau=T_{1}, T_{2},
$$

from the relaxation distribution and presented in Table 3. The average relaxation time is closely related to the pore structures of the media. Figure 3 shows that the relaxation rate of the reservoir samples are significantly greater than that of the Bentheimer sample on average. This implies that the pore structure of the Bentheimer sample is significantly different from the reservoir samples. 


\begin{tabular}{||c|r|r||}
\hline \hline Sample & $T_{2}^{\text {avg }}(\mathrm{ms})$ & $T_{1}^{\text {avg }}(\mathrm{ms})$ \\
\hline \hline R138 & 3.10 & 265 \\
R145 & 2.82 & 165 \\
\hline R221 & 4.14 & 156 \\
\hline R237 & 4.98 & 240 \\
\hline BENT1 & 17.13 & 346 \\
\hline
\end{tabular}

Table 3: Determined average relaxation times of the samples

\subsection{Conclusions}

A new data-driven nonparametric regression method is presented to estimate NMR relaxation distribution functions from NMR relaxation data. Statistical criteria are used to determine the optimal regularization parameter subject to equality and inequality constraints associated with the distribution function.

We validated the method with simulated data that were generated by a given true distribution function with different levels of measurement errors. The estimated function was consistent with the true function for all sets of data. The regularization term effectively worked so that the measurement errors did not significantly affect the estimation. All three statistical criteria examined provided similar values for the regularization parameters and the corresponding relaxation distribution functions.

The $T_{1}$ and $T_{2}$ relaxation distributions of several sandstone samples were estimated from relaxation experiments. The average relaxation times were evaluated from the relaxation distributions. The results showed that the relaxation rates associated with the reservoir samples are significantly greater than those for the Bentheimer sample. 


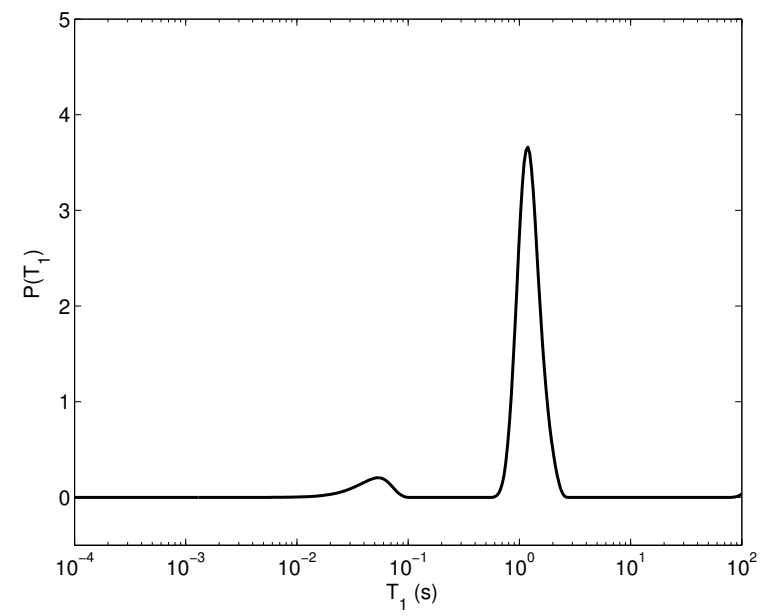

(a) $T_{1}$

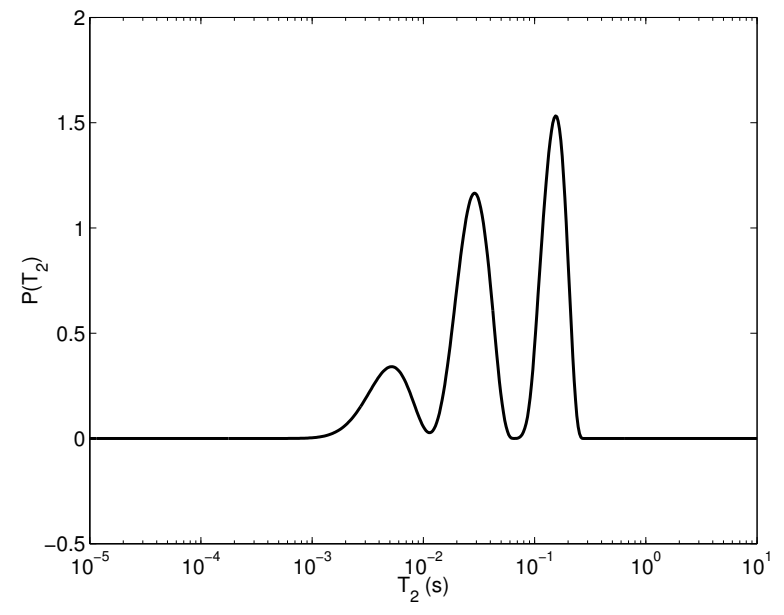

(b) $T_{2}$

Figure 8: NMR relaxation distributions of the Bentheimer sample (BENT1) 


\section{Determination of Surface Relaxivity and Pore-Size Distribu- tions}

The surface relaxivity is important for developing empirical correlations for improved permeability prediction. The surface relaxivity is also required to obtain pore-size distribution functions from the relaxation distributions. However, current methods to determine surface relaxivity is problematic. We develop a new method that extends the usefulness of NMR experiments for determining the surface relaxivity.

\subsection{Introduction}

One of the important porous media properties that NMR provides is the pore surface-to-volume ratio distribution, which is conventionally called pore-size distribution. With the "fast-exchange" approximation and isolated pore assumption (Brownstein and Tarr 1977), the distribution of NMR relaxation rates observed can be directly scaled to the pore-size distribution. The methodology for determining NMR relaxation distribution is described in Section 3. The issue in this section is how to determine the scaling factor to obtain pore-size distribution.

Several studies have proposed to determine an average pore-size from independent experiments, including mercury porosimetry (Gallegos et al. 1987), thin-section analysis (Howard et al. 1990), and gas adsorption (Borgia et al. 1996), to scale the relaxation distribution. However, NMR offers a number of advantages compared to those methods. Pulsed-field-gradient-stimulated-echo (PFGSTE) experiments can be used to determine the surface relaxivity-the NMR relaxation rate at the fluid-solid interface-which provides an intrinsic scale for specifying the pore-size distribution (Hürlimann et al. 1994; Latour et al. 1995; Fordham et al. 1994; Liaw et al. 1996).

These reported NMR methods are based on an asymptotic relationship for molecular selfdiffusion occurring at vanishingly short time. We have determined that such small times may not be achieved for many experiments of interest. We present a new method for obtaining NMR surface relaxivity from PFGSTE experiments that is free from restrictions on the experimental times.

\subsection{Scaling relaxation distribution to pore-size distribution}

Brownstein and Tarr (1977) proposed that $T_{1}$ for a fluid in a pore at the fast-exchange limit can be expressed in terms of $T_{1 b}$ and $T_{1 s}$, which are the relaxation times in the bulk phase and in the 
pore surface layer, respectively:

$$
\frac{1}{T_{1}}=\left[1-\eta\left(\frac{S}{V}\right)_{\text {pore }}\right] \frac{1}{T_{1 b}}+\eta\left(\frac{S}{V}\right)_{\text {pore }} \frac{1}{T_{1 s}},
$$

where $\eta$ is the thickness of the surface layer and $(S / V)_{\text {pore }}$ is the surface-to-volume ratio of the pore. Relaxation on pore surfaces is much faster than that of the bulk phase, because of the interactions between the solid and fluid molecules in the immediate vicinity of surfaces, i.e., $T_{1 b}>>T_{1 s}$. Then, Eq. 39 can be approximated as

$$
\frac{1}{T_{1}}=\frac{\eta}{T_{1 s}}\left(\frac{S}{V}\right)_{\text {pore }}=\rho_{s r}\left(\frac{S}{V}\right)_{\text {pore }} .
$$

Here, $\eta / T_{1 s}$ is defined as surface relaxivity $\rho_{s r}$. The surface relaxivity represents the strength of the relaxation on pore surfaces, and it depends on the properties specific to the solid surface and the fluid. A uniform value for the surface relaxivity is reasonable regardless of whether chemical composition of the solid material is uniform within a pore (Kleinberg et al. 1994). Spatial variations in chemical composition across the medium could lead to spatial variations in the surface relaxivity. One could, in principle, use spatially resolved NMR experiments to determine such spatial variations. Here, we assume the surface relaxivity is uniform.

Equation (40) implies that a $T_{1}$ distribution can be converted to the corresponding pore-size distribution through surface relaxivity. In this work, we define effective pore-radius as

$$
a=\frac{3}{(S / V)_{\text {pore }}} .
$$

The pore-size distribution $\tilde{P}(a)$ in the following refers to the distribution of effective pore-radius. Once $\rho_{s r}$ is determined the relaxation distribution can be scaled to the pore-size distribution by

$$
a=3 \rho_{s r} T_{1}
$$

which is derived from Eqs. (40) and (41).

\subsection{Determination of surface relaxivity using PFGSTE experimental data}

The surface relaxivity can be related to the sample surface-to-volume ratio, $S / V$, as follows: The average relaxation time, $T_{1}^{a v g}$, is defined as the harmonic mean of $T_{1}$ weighted by the number of nuclei corresponding to each value of $T_{1}$ :

$$
\frac{1}{T_{1}^{a v g}}=\sum_{j=1}^{N_{p}} \frac{1}{T_{1 j}} \frac{n_{j}}{N}=\int_{0}^{\infty} \frac{1}{\tau} P(\tau) d \tau
$$


Assuming $\rho_{s r}$ is uniform, we obtain the following from Eq. 40:

$$
\rho_{s r} \frac{1}{T_{1}^{a v g}}=\sum_{j=1}^{N_{p}} \rho_{s r} \frac{1}{T_{1 j}} \frac{n_{j}}{N}=\sum_{j}^{N_{p}} \frac{S_{j}}{V_{j}} \frac{n_{j}}{N} .
$$

Here, $S_{j}$ and $V_{j}$ represent surface area and pore volume of pore $j$, respectively. Given that $n_{j} / N=$ $V_{j} / V$

$$
\rho_{s r} \frac{1}{T_{1}^{a v g}}=\sum_{j=1}^{N_{p}} \frac{S_{j}}{V}=\frac{S}{V} .
$$

Thus, $\rho_{s r}$ can be calculated from the sample values $S / V$ and $T_{1}^{a v g}$. In this section, we review the use of PFGSTE to determine $S / V$ and surface relaxivity.

In the PFGSTE sequence, the molecular displacement due to self-diffusion during the time, $\Delta$, is encoded by the two magnetic gradient pulses. The details of PFGSTE sequence are described in Section 6, where it is used for velocity imaging. In this section, we are interested in detecting the self-diffusion of molecules using PFGSTE. The same basic pulse sequence that is used for velocity imaging is applied here, although there are some differences in the parameters associated with the pulse sequence and in the interpretation of the measured signal.

The self-diffusion of molecules in restricted geometries can be described by apparent diffusivity. The apparent diffusivity is defined in terms of mean-square displacement of molecules by

$$
D^{a p p}(t)=\frac{<[\mathbf{r}(t)-\mathbf{r}(0)]^{2}>}{6 t}
$$

where $\mathbf{r}(t)$ is the position of each spin at time $t$ and the angular brackets indicate the ensemble average. Mitra and Sen (1992) have shown that the apparent diffusivity can be evaluated from PFGSTE data, $M(q, \Delta)$, according to

$$
D^{a p p}(\Delta)=-\frac{1}{\Delta} \lim _{q \rightarrow 0} \frac{\partial \ln [M(q, \Delta) / M(0, \Delta)]}{\partial\left(q^{2}\right)}
$$

where $q=\delta \gamma G$ and $\gamma$ is gyromagnetic ratio. In porous media, the apparent diffusivity decreases from the value of bulk diffusivity, $D_{0}$, as $\Delta$ increases because of restrictions to diffusion posed by the solid surfaces. Mitra etal. (1992) expanded $D^{a p p}(\Delta)$ as a polynomial series in terms of $\sqrt{\Delta}$. The "short-time approximation" is given by the first order term:

$$
\frac{D^{a p p}(\Delta)}{D_{0}} \simeq 1-\frac{4}{9 \sqrt{\pi}}\left(\frac{S}{V}\right) \sqrt{D_{0} \Delta}
$$

The sample surface-to-volume ratio, $S / V$, has been determined from PFGSTE data using Eqs. (47) and (48) (Fordham et al. 1994; Liaw et al. 1996). To this end, PFGSTE data are measured 


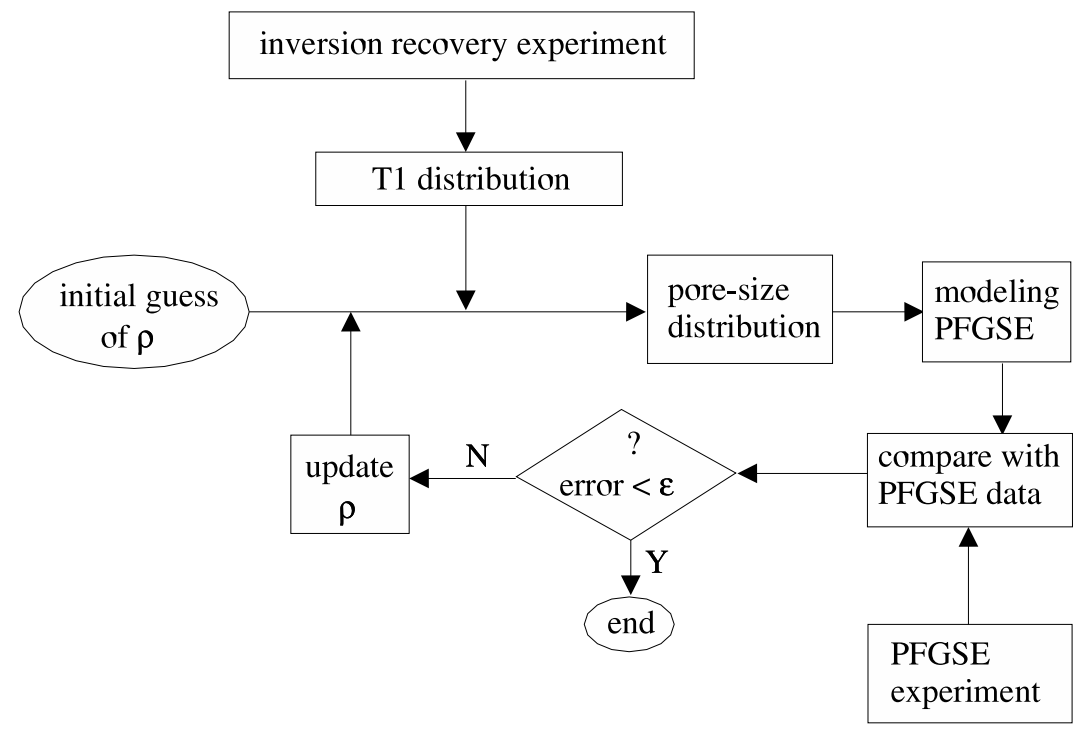

Figure 9: Procedure of the proposed method for determination of surface relaxivity

with a series of different gradient strengths and diffusion times, and the apparent diffusivities are evaluated as a function of diffusion time. The PFGSTE data must be interpolated to find the differential value, as $q \rightarrow 0$, in Eq. 47. Then, $S / V$ is determined by the linear relationship between $D^{a p p}(\Delta)$ and $\sqrt{\Delta}$ given by Eq. 48. However, the measurements corresponding to the range of time over which the asymptotic representation is valid cannot be achieved in some PFGSTE experiments, particularly when samples exhibit relatively fast relaxation.

In an effort to extend the utility of this approach, Hürlimann et al. (1994) used a two-point Padé approximation introduced by Latour et al. (1993) to interpolate between the short and long time asymptotic relations. Consequently, the interpolated equation includes parameters associated with the two asymptotic equations and an additional fitting parameter. The determination of $S / V$ from the interpolated equation involves identification of those parameters, but this requires additional experiments, such as permeability measurement (Hürlimann et al. 1994).

In our method, we mathematically model the PFGSTE experiment over the full range of experimental time. Here, the PFGSTE response from a sample with a given pore-size distribution is represented in terms of the strength of applied gradient pulse and diffusion time. Once the PFGSTE experiment is modeled, the surface relaxivity is determined from the measured stimulated echo data through an inverse problem. 
Figure 9 illustrates how this problem is solved. The inversion recovery experiment is performed and the $T_{1}$ relaxation distribution is estimated. The surface relaxivity is initially postulated, and $P(\tau)$ is scaled to $\tilde{P}(a)$. The stimulated echo calculated from the model, $M^{\text {calc }}(q, \Delta)$, is compared with experimental data, $M^{o b s}(q, \Delta)$, and the surface relaxivity is updated until it minimizes the performance index

$$
J=\left[\left(\frac{M^{o b s}(q, \Delta)}{M^{o b s}(0, \Delta)}\right)-\left(\frac{M^{c a l c}(q, \Delta)}{M^{c a l c}(0, \Delta)}\right)\right]^{2} .
$$

Then, the pore-size distribution can be obtained by the determined surface relaxivity and the relaxation distribution.

It should be noted that this approach avoids the need for defining and using time-dependent apparent diffusivities and estimating derivatives from discrete data-often a source of large errors-as done in previous methods. Furthermore, the entire set of measured data may be used, rather than just those values corresponding to sufficiently "short" times, if they are indeed obtainable.

At longer times, however, additional features of the structure of the permeable media, aside from just the value of $S / V$, become important. One could consider a hierarchy of mathematical models of increasing complexity, depending on the manner in which the structure of the media is represented. We consider here one of the simpler models that still allows for an entire distribution of pore-sizes.

We assume that pores are isolated, and of spherical shape. Note that current methods used to estimate pore-size distributions from NMR experiments are based on the assumption of isolated pores. To meet this assumption, the data should not be significantly affected by inter-pore diffusion. The effect of inter-pore coupling has been investigated by McCall et al. (1991). They simulated decay rates using an idealized network model containing pores and throats and demonstrated that the spectra of decay rates narrows as the degree of inter-pore coupling increases. They speculated that inter-pore coupling is probably not significant for sandstones but could be significant for solgel glasses having very large porosities $(\sim 85 \%)$. Latour et. al. (Latour et al. 1992) investigated whether inter-pore coupling was significant for various water-saturated rock samples. They showed that the relaxation time distributions are not sensitive to the temperature changes ranging from 25 to $175^{\circ} \mathrm{C}$, implying that the effect of pore connectivity is not significant (Kleinberg 1994) and the isolated pore model is valid in describing such samples. These studies considered the entire range of time associated with spin-lattice relaxation. It should be noted that the diffusion times associated with the PFGSTE experiment can likely be selected sufficiently small to ensure that the isolated pore assumption is always met. 
The stimulated echo for a sample having pore-size distribution $\tilde{P}(a)$ is represented by the integral

$$
M(q, \Delta)=\int_{0}^{\infty} \tilde{P}(a) M(a ; q, \Delta) d a
$$

where $M(a ; q, \Delta)$ is the magnetization for pores of size $a$. The stimulated echo signal $M(\mathbf{q}, \Delta)$ can be expressed as

$$
M(q, \Delta)=M_{0} \iint \bar{G}\left(\mathbf{r}, \mathbf{r}^{\prime}, \Delta\right) \exp \left[-i \mathbf{q} \cdot\left(\mathbf{r}-\mathbf{r}^{\prime}\right)\right] d \mathbf{r} d \mathbf{r}^{\prime}
$$

Here, $M_{0}=M(q=0, \Delta=0)$. The usual short gradient pulse approximation (Tanner and Stejskal 1968), in which the gradient pulse widths are assumed much smaller than the diffusion time $(\delta \ll$ $\Delta)$, is used. The propagator $\bar{G}\left(\mathbf{r}, \mathbf{r}^{\prime}, \Delta\right)$ represents the probability that a spin initially at point $\mathbf{r}$ moves to location $\mathbf{r}^{\prime}$ during the time $\Delta$. This satisfies the relation

$$
\frac{\partial \bar{G}\left(\mathbf{r}, \mathbf{r}^{\prime}, t\right)}{\partial t}=D_{0} \nabla^{2} \bar{G}\left(\mathbf{r}, \mathbf{r}^{\prime}, t\right), \quad t>0
$$

with the initial condition,

$$
\bar{G}\left(\mathbf{r}, \mathbf{r}^{\prime}, 0\right)=\hat{\delta}^{3}\left(\mathbf{r}-\mathbf{r}^{\prime}\right)
$$

and the boundary condition

$$
D_{0} \mathbf{n} \cdot \nabla \bar{G}\left(\mathbf{r}, \mathbf{r}^{\prime}, \Delta\right)+\left.\rho_{s r} \bar{G}\left(\mathbf{r}, \mathbf{r}^{\prime}, \Delta\right)\right|_{\text {surfaces }}=0
$$

where $\hat{\delta}$ is the Dirac delta function and $\mathbf{n}$ is the vector normal to the pore surfaces.

The solution of Eqs. (51)-(54) can be obtained analytically for simple geometries such as planar, cylindrical and spherical pores (Mitra and Sen 1992; Snaar and As 1993; Coy and Callaghan 1994; Callaghan 1995). We use the spherical pore model since it is the most reasonable one to describe three-dimensional pore space. The stimulated echo of a single spherical pore with radius $a$ is written as (Mitra and Sen 1992; Callaghan 1995)

$$
M(a ; q, \Delta)=M_{0} \sum_{n=0}^{\infty} \sum_{l=0}^{\infty} \frac{6(2 l+1) \zeta_{l n}^{2} \exp \left(-D_{0} \Delta \zeta_{l n}^{2} / a^{2}\right)}{\left(\zeta_{l n}^{2}-q^{2} a^{2}\right)^{2}} \frac{\left(q a j_{l}^{\prime}(q a)+\rho_{s r} a / D_{0} j_{l}(q a)\right)^{2}}{\left(\rho_{s r} a / D_{0}-\frac{1}{2}\right)^{2}+\zeta_{l n}^{2}-\left(l+\frac{1}{2}\right)^{2}}
$$

Here, $j_{l}$ is a spherical Bessel function of order $l$ and the eigenvalue $\zeta_{l n}$ is the $n$th root of the equation

$$
\zeta_{l n} j_{l}^{\prime}\left(\zeta_{l n}\right)=-\frac{\rho_{s r} a}{D_{0}} j_{l}\left(\zeta_{l n}\right)
$$

We note that numerical solutions could be used if more complex shapes, or even coupled pores systems, were desired. 


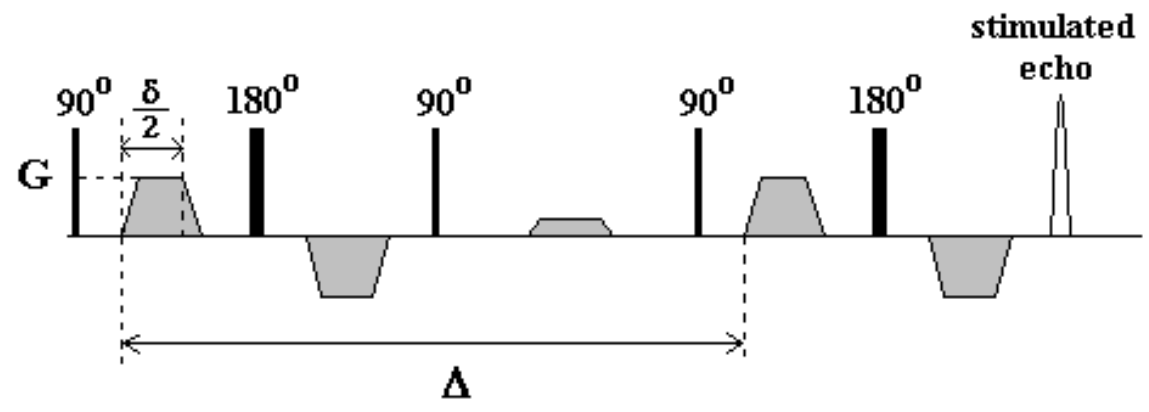

Figure 10: The "13-interval, Condition I" pulsed field gradient stimulated echo pulse sequence

\subsection{Experiment}

A PFGSTE experiment was performed on the Bentheimer sample (BENT1) to determine its surface relaxivity. We use a pulse sequence based on the "13-interval, Condition I" sequence of Cotts et al. (1989) (Fig. 10), which is designed to reduce the errors due to background magnetic gradient arising from the magnetic susceptibility contrast in the porous media. Compared to the Stejskal and Tanner (1965) sequence, the pulse sequence has alternating gradient directions with additional $180^{\circ} \mathrm{RF}$ pulses in dephasing and rephasing stages. As a result, the phase changes due to the background gradient are refocused. In stimulated echo sequences, unwanted spin-echoes are also generated and often overlap with the stimulated echoes. This situation becomes more complicated when a greater number of RF pulses are applied. We used phase cycling and spoiler gradients (Fordham etal. 1994; Liaw et al. 1996) to mitigate this problem so that the desired stimulated echo is accumulated preferentially. The gradient pulses are designed in trapezoidal shape, which makes $q=\gamma \delta G$, with the maximum amplitude of $G$ and width of $\delta / 2$ for each gradient pulse. The gradient pulse width $\delta$ was $2 \mathrm{~ms}$. The PFGSTE experiment was performed with a series of gradient pulses from $G=$ $0 \mathrm{G} / \mathrm{cm}$ to $19 \mathrm{G} / \mathrm{cm}$ for each of the four different diffusion times: $\Delta=20 \mathrm{~ms}, 30 \mathrm{~ms}, 50 \mathrm{~ms}$, and $100 \mathrm{~ms}$.

\subsection{Results and discussion}

Figure 11 shows the measured PFGSTE data and the calculated values with the estimated surface relaxivity. The surface relaxivity was estimated using the $T_{1}$ relaxation distribution of the sample (Fig. 8a). The result of the estimation is summarized in Table 4. Here, the average pore-radius $a^{a v g}$ is the effective radius corresponding to the overall $S / V$ evaluated by $a^{a v g}=3 /(S / V)$ and 


\begin{tabular}{|c|c|}
\hline \hline Sample & BENT1 \\
\hline \hline Number of data & 80 \\
\hline RMSE & 0.0504 \\
\hline$\rho_{s r}(\mu \mathrm{m} / \mathrm{s})$ & $18.6 \mu \mathrm{m} / \mathrm{s}$ \\
\hline$a^{a v g}(\mu \mathrm{m})$ & $19.3 \mu \mathrm{m}$ \\
\hline$S / V\left(\mu \mathrm{m}^{-1}\right)$ & $0.16 \mu \mathrm{m}^{-1}$ \\
\hline \hline
\end{tabular}

Table 4: Results of surface relaxivity estimation for reservoir samples

$S / V=1 /\left(\rho_{s r} T_{1}^{a v g}\right)$. Figure 11 and the value of root-mean-square error (RMSE) indicate a good fit to the experimental data, validating the model used in this study. The $T_{1}$ distribution is converted to the pore-size distribution using the determined surface relaxivity (Fig. 12).

It is instructive to investigate the range of time over which the short-time approximation holds. If we suppose that the model and estimated distribution are correct, we can use Eq. 47 to calculate the apparent diffusivity for any time $\Delta$. This is plotted in Fig. 13. Note that these simulated values are consistent with values calculated directly from the measured data (also shown in Fig. 13). The short-time approximation given by Eq. 48, with our estimated value of $S / V$, is plotted in Fig. 13 . Note that its slope is consistent with that of the simulated curve as $\Delta \rightarrow 0$. The figure indicates that the short-time approximation is valid for $\sqrt{D_{0} \Delta}<2 \mu \mathrm{m}$. However, that approximation is not useful for the interpretation of the data since there are no data within that range of time.

The PFGSTE method for determination of surface relaxivity is applied to the reservoir samples. The results of the surface relaxivity estimation are presented in Table 5. The table shows that while the data from R238 are fitted by the model as well as the Bentheimer sample, the data from the other two samples are not. This deviation probably arises from the assumptions associated with the model such as fast-exchange approximation and spherical pores. While the model can be improved by considering more precise simulation of the NMR experiment, it should be noted that the surface relaxivity could not have been evaluated in other ways using the given PFGSTE data. Tables 4 and 5 show that the average pore size of the Bentheimer sample is larger than those of the reservoir samples while their surface relaxivity values are approximately in the same order of magnitude. 


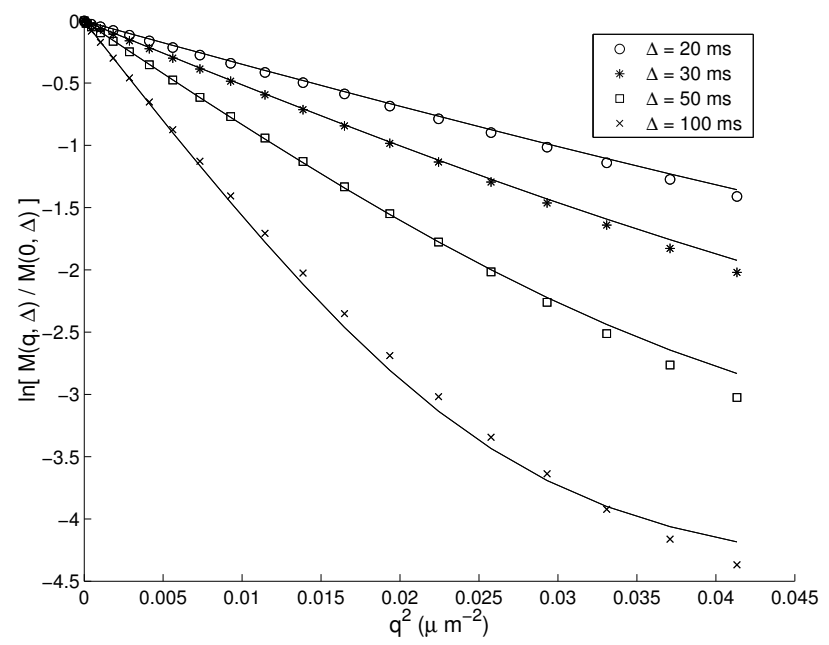

Figure 11: PFGSTE data and calculated values

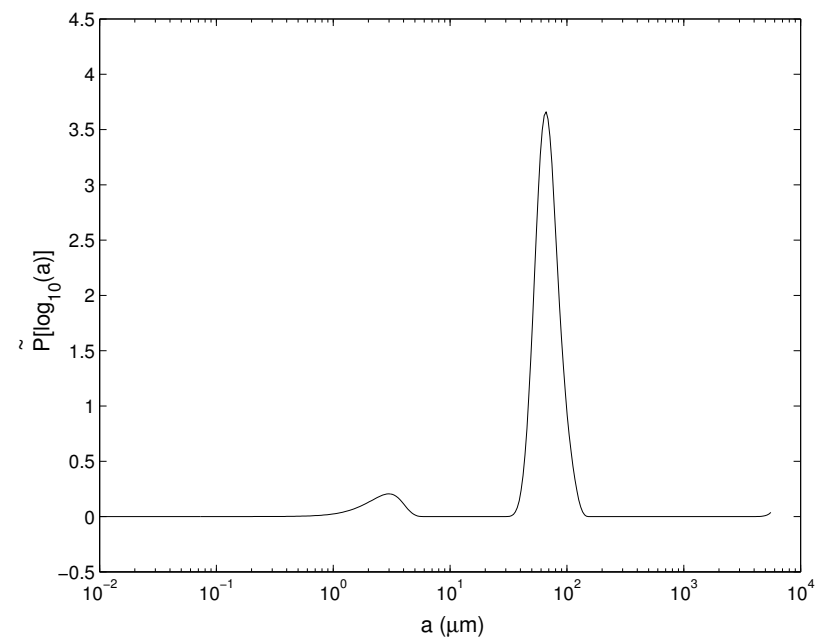

Figure 12: Determined pore-size distribution 


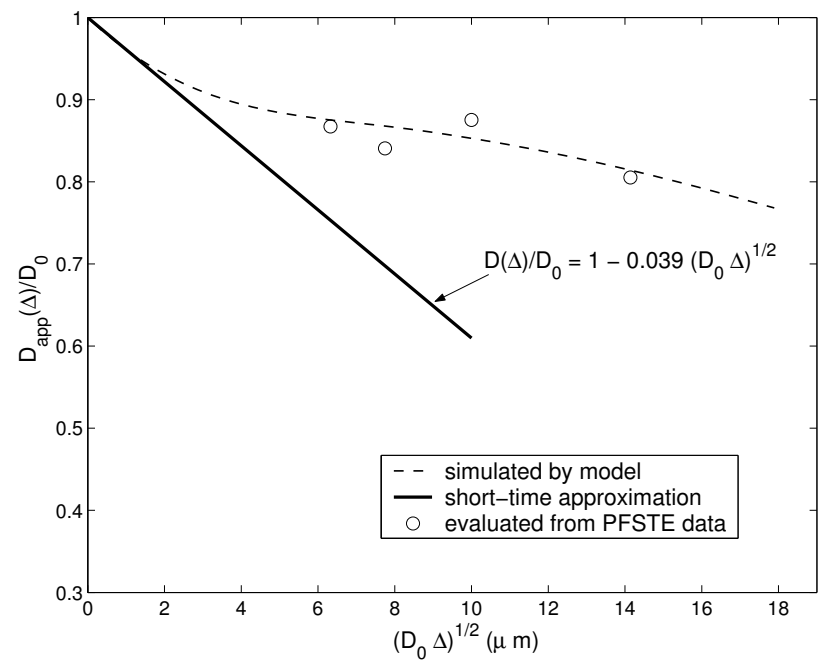

Figure 13: Apparent diffusivities and short-time approximation

\begin{tabular}{|c|c|c|c||}
\hline \hline Sample & $\mathrm{R} 238$ & $\mathrm{R} 138$ & $\mathrm{R} 145$ \\
\hline \hline Number of data & 44 & 80 & 80 \\
\hline $\mathrm{RMSE}$ & 0.0181 & 0.142 & 0.134 \\
\hline$\rho_{s r}(\mu \mathrm{m} / \mathrm{s})$ & 44.0 & 22.6 & 14.7 \\
\hline$a^{\text {avg }}(\mu \mathrm{m})$ & 9.9 & 7.4 & 3.0 \\
\hline$S / V\left(\mu \mathrm{m}^{-1}\right)$ & 0.30 & 0.41 & 1.0 \\
\hline \hline
\end{tabular}

Table 5: Results of surface relaxivity estimation for reservoir samples (The experimental data for R238 were measured with General Electric 2T Omega CSI system) 


\subsection{Conclusions}

A new method was developed and validated to determine surface relaxivity for use in estimating pore-size distributions and developing correlation to predict permeability. The PFGSTE experiment is mathematically modeled and the surface relaxivity is found by minimizing the differences between the calculated and measured echo data. The new method avoids restrictions on experimental times which are integral in previous methods, and it does not require estimates of derivatives of measured data, which can be a significant source of errors.

The new method is demonstrated with experiments performed on Bentheimer and reservoir sandstone samples. Examination of the differences between measured data and the corresponding calculated values showed that a more precise reconciliation of the data was achieved with Bentheimer sandstone. It gave a precise fit to the experimental PFGSTE data, from which surface relaxivity and the corresponding pore-size distribution are obtained. The surface relaxivity from reservoir samples are also determined and evaluated. 


\section{Determination of Porosity and Saturation Distributions}

The net magnetization at equilibrium is proportional to the number of NMR-active nuclei, thus providing, in principle, the means to determine the amount of fluid, and consequently the porosity or saturation. However, the equilibrium state is not observed directly and must be inferred from dynamic observations that include relaxation effects. Here we develop methodology to estimate the intrinsic (equilibrium) magnetization intensity so that accurate assessments of porosity and saturation distributions can be obtained.

\subsection{Introduction}

Porosity and fluid saturation are fundamental properties used to characterize multiphase flow in porous media. Quantitative measurements of these distributions are essential for describing the amount of fluid phases present within local regions. The interpretation of NMR relaxation data allows the accurate quantification of the relative amount of the observed fluid, as a function of position, throughout the samples of permeable media. When a single fluid phase fills the pore space, the porosity distribution can be computed. When multiple fluid phases are present, each fluid saturation can be determined.

In this project, we have developed methodologies for the quantification of porosity and saturation distributions in porous media in up to three spatial dimensions. Experiments are carried out by detecting the NMR signals from protons in the fluid molecules. The use of NMR imaging techniques to determine porosity and saturation distributions is based on the principle that the intrinsic image signal intensity is proportional to the number of protons in the corresponding volume element of the sample. The main challenge in quantification arises from the fact that the intrinsic nuclear magnetization is not actually measured since imaging sequences have a certain minimum time from excitation of the spin system to acquiring image data. Thus, some relaxation occurs before data are acquired. For fluids in porous media, the relaxation is significantly enhanced due to the fluid/solid interaction and diffusion of fluid molecules in the presence of magnetic-susceptibilityderived field gradients. The relaxation rate is sensitive to the pore size and fluid saturation, as well as local surface compositions. To obtain quantitative values of porosity and saturation in a

heterogeneous sample, it is essential to consider the spatial variation of the relaxation effects on the observed image signals. By measuring the signals corresponding to different degrees of relaxation, the intrinsic magnetization intensity can be estimated for each volume element, or voxel. 

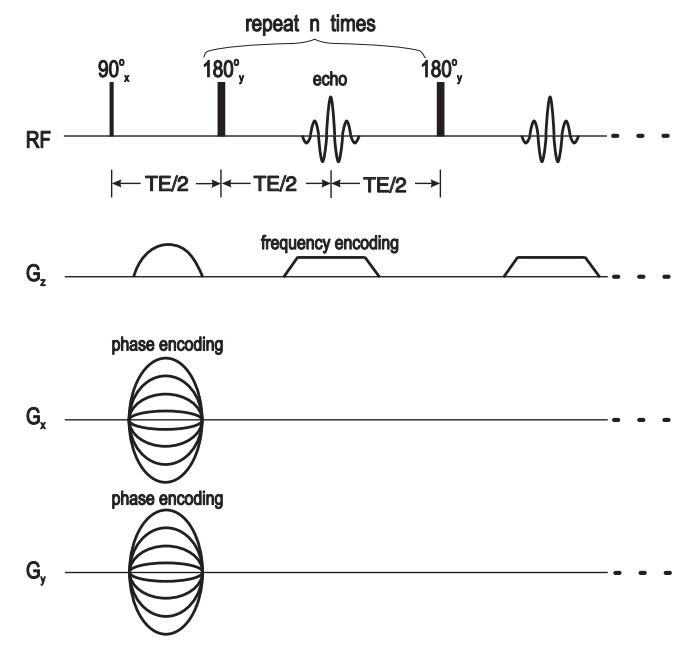

Figure 14: An example of three-dimensional CPMG imaging pulse sequence.

\subsection{Methodology}

We use a CPMG imaging pulse sequence incorporated with spatial encodings (Fig. 14) to obtain $T_{2}$ relaxation data at each voxel. The spatial information is frequency-encoded in one dimension and phase-encoded in the other two dimensions.

The relaxation distribution is estimated for each voxel as described in Section 3, where the individual regularization parameter for each voxel is sought. Once the (non-normalized) relaxation distribution of a voxel $i, \mathcal{P}_{i}(\tau)=M_{0 i} P_{i}(\tau)$, is determined, the corresponding intrinsic magnetization, $M_{0 i}$ can be easily calculated by

$$
M_{0 i}=\int_{0}^{\infty} \mathcal{P}_{i}(\tau) d \tau
$$

Then, the mass of fluid in voxel $i, m_{i}$, is related to $M_{0 i}$ by

$$
M_{0 i}=c_{m} m_{i}
$$

where $c_{m}$ is the specific magnetization intensity.

The value of $c_{m}$ is affected by several experimental quantities, such as the receiver gain, the static magnetic field, and the quality factor-a measure of the energy losses in the receiving coil. While the receiver gain may change only slightly with time during an experiment, the quality factor may change significantly (Abragam 1961; Mandava et al. 1990). The specific magnetization intensity can be determined by placing a reference standard containing a known quantity of fluid into the imaged volume next to the sample (Chang et al. 1993). During the experiment, both the 
sample and reference standard experience the same receiver gain and quality factor, so that the value of $c_{m}$ for both is the same throughout the experiment. An alternative way of determining specific magnetization is to use the amount of fluid in the sample if it can be accurately measured.

The porosity is determined by imaging a fully saturated sample with an NMR active fluid such as water. The fluid is assumed to completely fill the void space. The fluid mass determined in Eq. 58 is then scaled to fluid volume using the fluid's density, $\rho$. The porosity for the $i$ th voxel is then given by

$$
\phi_{i}=\frac{M_{0 i}}{k_{m} \rho V_{i}},
$$

where $V_{i}$ is the volume of the $i$ th voxel. The saturation distribution is determined from a sample saturated with multiple phases of fluids. The procedure for determination of two-phase saturation distributions is essentially the same as that of porosity distribution when the signal from one of the fluids is suppressed. We use two fluids such as $\mathrm{D}_{2} \mathrm{O}$ and oil for which Lamar frequencies are different to selectively detect one phase: The probe is tuned to one of them, in our work, oil, so that the signal from the other fluid $\left(\mathrm{D}_{2} \mathrm{O}\right)$ is not detected. The saturation of a phase $j$ in a voxel $i$ is calculated by

$$
\left(S_{j}\right)_{i}=\frac{\left(M_{0 j}\right)_{i}}{k_{m} \rho_{j} V_{i} \phi_{i}}
$$

where $\left(M_{0 j}\right)_{i}$ is the intrinsic magnetization of phase $j$ in voxel $i$ and $\rho_{j}$ is the density of the phase.

\subsection{Experimental results and discussion}

In this section, we demonstrate our experimental works to determine porosity distributions for the Bentheimer and reservoir samples (Table 2). The procedure for determination of saturation distributions is not presented here in understanding that it is essentially the same as that for determination of porosity distributions. Experimentally resolved saturation distributions presented in Section ??.

The three-dimensional CPMG imaging pulse sequence (Fig. 14) was implemented in ParaVision ${ }^{\circledR}$, which is the pulse programming and data acquisition software system equipped with the NMR imager. The key parameter values associated with the imaging sequence are given in Table 6 . The post processing of the raw data, including Fourier transforms, were performed in MATLAB ${ }^{\circledR}$.

The NMR $T_{2}$ relaxation data for each voxel were analyzed to obtain the corresponding relaxation distribution and the intrinsic magnetization. The relaxation distribution was represented by cubic B-spline basis functions with a partition of 100 interior knots logarithmically spaced between 


\begin{tabular}{||c||c|c|}
\hline \hline \multicolumn{1}{|c||}{ Sample } & Reservoir samples & Bentheimer (BENT1) \\
\hline \hline Spectral width & $50000 \mathrm{~Hz}$ & $50000 \mathrm{~Hz}$ \\
\hline Radio-frequency(RF) pulse length & $50 \mu \mathrm{s}$ & $50 \mu \mathrm{s}$ \\
\hline Repetition time & $5 \mathrm{~s}$ & $1 \mathrm{~s}$ \\
\hline Echo spacing & $3.06 \mathrm{~ms}$ & $4.6 \mathrm{~ms}$ \\
\hline Number of echoes & 32 & 50 \\
\hline Field of view & $64 \times 32 \times 32 \mathrm{~mm}^{3}$ & $30 \times 30 \times 30 \mathrm{~mm}^{3}$ \\
\hline Matrix size & $32 \times 16 \times 16$ & $32 \times 16 \times 16$ \\
\hline Voxel size & $2.0 \times 2.0 \times 2.0 \mathrm{~mm}^{3}$ & $0.938 \times 1.875 \times 1.875 \mathrm{~mm}^{3}$ \\
\hline \hline
\end{tabular}

Table 6: Parameters of CPMG imaging sequences

$1 \times 10^{-5}$ and 10 seconds. The optimal regularization parameter of each voxel was found by the UBR criterion. The intrinsic magnetization was scaled to porosity value using the bulk porosity.

The CPMG relaxation data and the corresponding analyzed results are exemplified for a voxel in Fig. 15. The position of the voxel is represented by the variables $Z_{1}, Z_{2}$, and $Z_{3}$, each of which numbers the voxel along the corresponding $z_{1^{-}}, z_{2^{-}}$, and $z_{3}$-directions. Here, we take the convention that $z_{1}$ is parallel with the static magnetic field, $z_{2}$ is horizontal, and $z_{3}$ is vertical. The solid line in the Fig. 15(a) stands for the predicted relaxation calculated by the determined relaxation distribution given in Fig. 15(b). The value of the line at $t=0$ represents the intrinsic magnetization. Figure 15(a) shows that the intrinsic magnetization significantly deviates from the first echo, which would not be accurately estimated unless the relaxation distribution is reliably estimated.

The porosity distribution (resolved in three dimensions) is obtained by scaling the intrinsic magnetization values. The porosity distribution is represented as a series of two-dimensional grayscale images in Fig. 16. Each image of the figure represents a horizontal slice of the sample, numbered from the bottom to the top. Since the field of view is larger than the sample size, there are regions in the images that do not belong to the sample. For those regions, the porosity is assigned to be zero and they are presented with black areas in the images.

While Fig. 16 shows the spatial distribution of the porosity, its number distribution can provide useful statistical information for understanding the characteristics of the porous media. The histogram of the porosities of the Bentheimer sample (BENT1) is given in Fig. 17 with the correspond- 


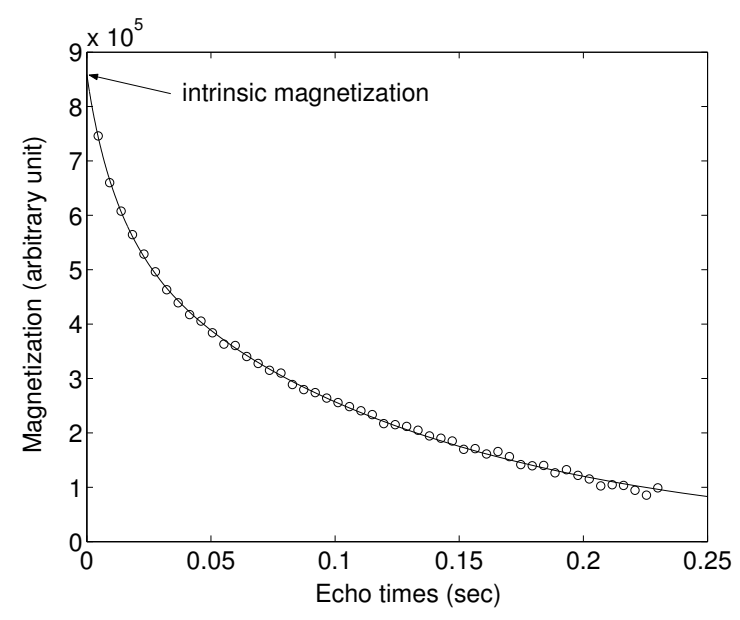

(a)

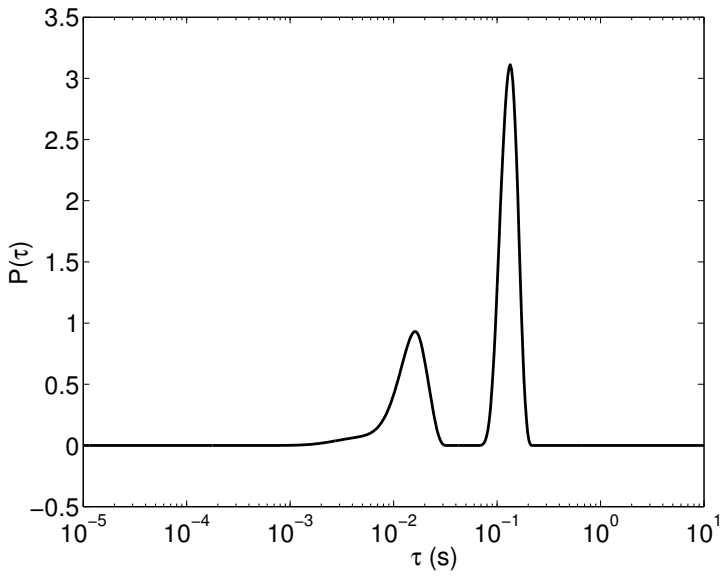

(b)

Figure 15: The relaxation data (a) and the estimatied distributiion function (b) of a voxel at $Z_{1}=$ $16, Z_{2}=9, Z_{3}=8$ in the Bentheimer sample (BENT1)

ing statistical analysis provided by MINTAB ${ }^{\circledR}$. The Anderson-Darling Normality Test (Stephens 1976; Stephens 1979) is used to test whether the porosity values are normally distributed. The null hypothesis is that the porosity distribution follows a normal distribution, and the test static, $A^{2}$, is given by

$$
A^{2}=\sum_{i=1}^{N_{v}} \frac{(2 i-1)}{N_{v}}\left[\ln F\left(\phi_{i}\right)+\ln \left(1-F\left(\phi_{N_{v}+1-i}\right)\right]-N_{v} .\right.
$$

Here, $F$ is the cumulative normal distribution function, $\phi_{i}$ are the ordered porosity data, and $N_{v}$ is the number of voxels. Stephens (1979) provides the critical values of $A^{2}$ for various significance levels. For instance, the hypothesis is rejected (i.e., the distribution is not normal) if $A^{2}>2.492$ for the significance level of $\alpha=0.05$. The skewness is a measure of the lack of symmetry and the kurtosis is a measure of whether the data are peaked or flat relative to a normal distribution. Generally speaking, the sample with larger absolute value of skewness or kurtosis deviates more from the normal distribution.

The porosity distributions for reservoir samples are presented in Appendix 1. Their statistical analyses are summarized in Table 7 . The $A^{2}$ values in the table show that the hypothesis of normal distribution is rejected for all the samples in the table with $\alpha=0.05$.

\subsection{Conclusions}

The porosity and saturation distributions in porous media can be determined by scaling the intrinsic magnetization of the saturating fluid, which is estimated from the relaxation measurements 


\begin{tabular}{||c|c|c|c|r|r|r|c|c|}
\hline \hline sample & mean & min. & max. & st.dev. & $A^{2}$ & skewness & kurtosis & \# of data \\
\hline \hline R138 & 0.240 & 0.038 & 0.782 & 0.120 & 13.69 & 0.581 & 0.135 & 2423 \\
\hline R145 & 0.227 & 0.034 & 0.559 & 0.090 & 3.26 & 0.314 & 0.111 & 2362 \\
\hline R207 & 0.223 & 0.056 & 0.486 & 0.077 & 9.35 & -0.141 & -0.517 & 2637 \\
\hline R221 & 0.189 & 0.023 & 0.567 & 0.101 & 15.36 & 0.442 & -0.305 & 2981 \\
\hline R237 & 0.213 & 0.032 & 0.577 & 0.073 & 4.26 & -0.023 & 0.199 & 2724 \\
\hline Bentheimer & 0.237 & 0.138 & 0.403 & 0.029 & 10.54 & -0.067 & 1.300 & 2826 \\
\hline \hline
\end{tabular}

Table 7: Statistical analysis on porosity distributions of various samples
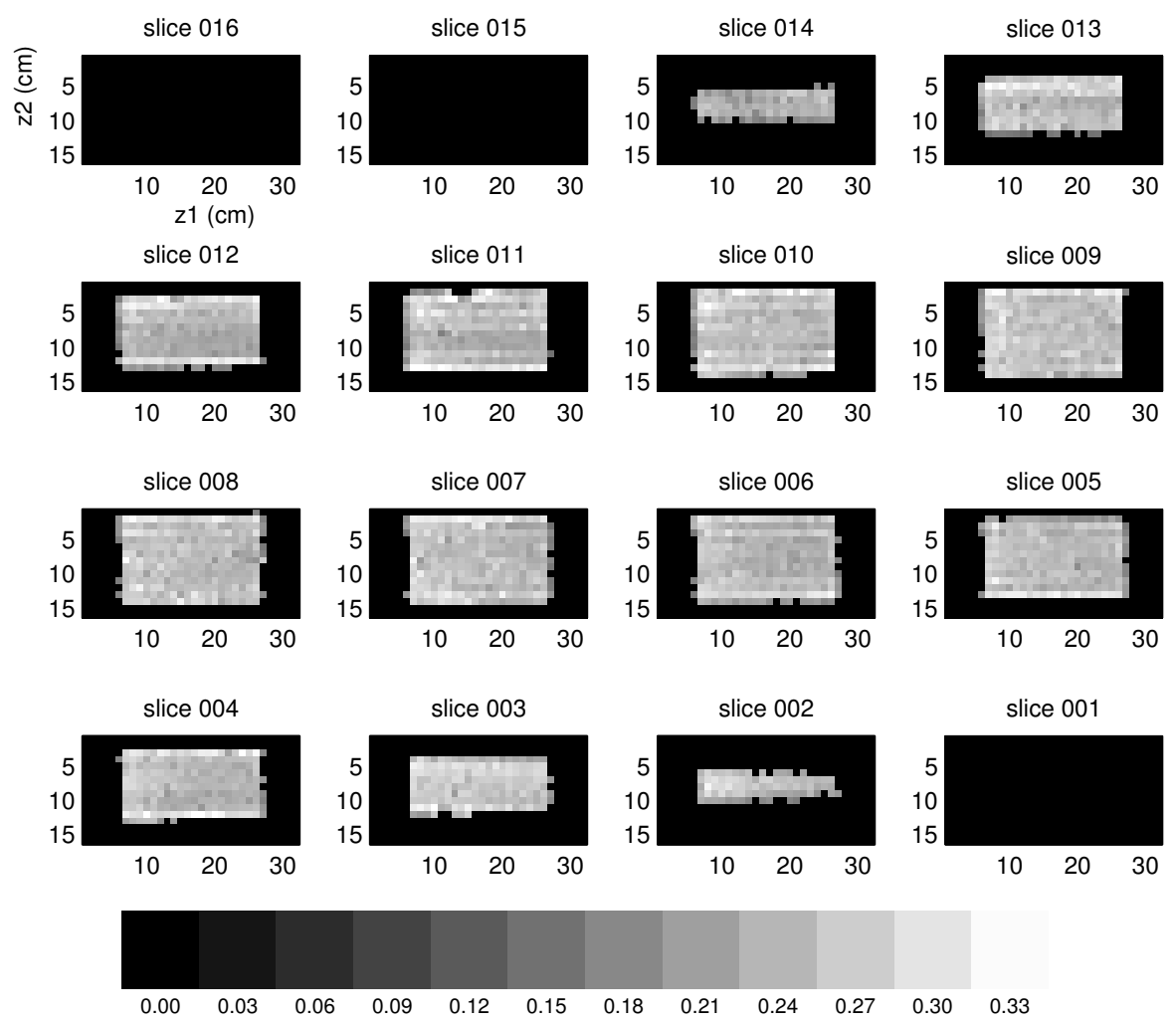

Figure 16: Three-dimensional porosity distribution of the Bentheimer sample 


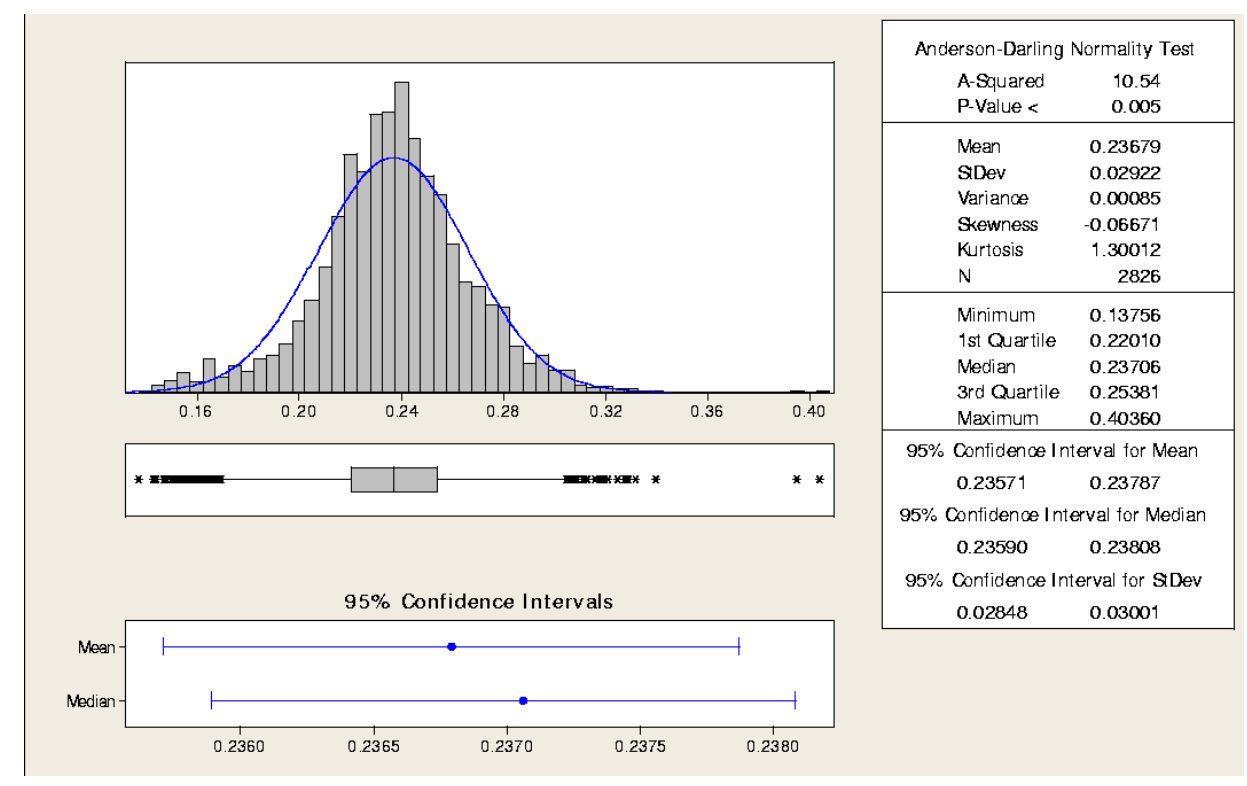

Figure 17: Porosity histogram of the Bentheimer sample and the corresponding statistical analysis

corresponding to each volume element. The spatial distributions of porosity obtained for a number of sandstone samples reveal the heterogeneous nature of the samples. Our statistical analysis reveals that the porosity of the examined samples do not follow normal distributions. 


\section{Determination of Permeability Distributions using Velocity Imag- ing Experiments}

While both X-ray CT and magnetic resonance imaging can provide the means to obtain spatially resolved information, NMR is unique in its sensitivity to molecular motion. In this work, we obtain measures of fluid velocity distributions during flow experiments, which we in turn use to determine the permeability distributions. We are unaware of any other method to resolve the permeability distributions within laboratory samples.

\subsection{Introduction}

While the porosity can be defined for a selected volume element, the permeability is essentially defined by Darcy's law-i.e., it represents the proportionality between the local volume-averaged velocity and gradient of the volume-averaged pressure (see Eq.2). This means that the actual determination of permeability must be accomplished using that equation. By setting up an experimental system which can be modeled with Darcy's Law, fluid states, or functions of the states, can be measured and permeability calculated using the model equations.

Conventional laboratory experiments provide only a single apparent permeability value of a sample, which will be a good measure of the permeability only if it is uniform throughout the sample (i.e., the sample is homogeneous). The lack of methodology for resolving spatial distributions of permeability has been a long-standing problem for understanding, measuring, and predicting that property.

NMR imaging provide a unique opportunity to observe molecular motions associated with diffusion or flow, which gives key measurements for determination of permeability distributions. while NMR has been used to image flow for decades (Packer etal. 1972; Garroway 1974), the reliable determination of fluid velocity within permeable media is problematic. Permeable media with pores that are smaller than an imaging voxel, as is the case in our studies, require particular considerations. The velocity varies throughout each pore and among pores, so that a wide range of velocities is represented within each voxel. Therefore, the average velocity in a voxel is not necessarily proportional to the average phase shift of spins, which might apply for flow in conduits

(Caprihan and Fukushima 1990). Instead, one must obtain the number distribution of velocity in a voxel and convert it to average velocity (Chang and Watson 1999) in order to obtain useful quantitative information. 
In this project, we developed an NMR velocity imaging methodology based on the pulsed field gradient stimulated-echo (PFGSTE) technique. The velocity number distributions are determined with three-dimensional spatial resolution. We also developed an associated estimation (inverse) problem to determine spatial distributions of permeability. The superficial average velocities calculated from the velocity number distributions are the essential input information to solve the inverse problem. In the following subsections, we describe our experimental work for measuring fluid velocities (Section 6.2), and illustrate the methodology of solving the corresponding estimation problem for determining the permeability distribution using the velocity data (Section 6.3).

\subsection{NMR velocity imaging}

\subsubsection{Methodology}

Pulsed-field-gradient-stimulated echo (PFGSTE) NMR detects molecular displacement over a well defined time scale and can be an excellent approach to the characterization of transport processes as well as structural features in porous media. The imaging of fluid molecular velocity can be achieved by using the pulsed field gradient method to encode the displacement of fluid molecules and coupling this with a suitable NMR spatial imaging sequence.

The PFGSTE NMR method is based on the observation that nuclear spins moving in the presence of a magnetic field gradient exhibit a phase shift in the transverse magnetization. For a time-dependent gradient, the phase shift of a spin is provided by

$$
\varphi(t)=\gamma \int_{0}^{t} \mathbf{G}\left(t^{\prime}\right) \cdot \mathbf{r}\left(t^{\prime}\right) d t^{\prime}
$$

In order to obtain a well-defined molecular displacement, it is advantageous to use intense gradient pulses whose pulse widths are much shorter than the time interval between them (Edwards et al. 1993). If the pulse width $\delta$ is sufficiently short, the phase shift during the gradient pulse is written as

$$
\varphi=\mathbf{q} \cdot \mathbf{r}
$$

Here, $\mathbf{q}$ is defined as

$$
\mathbf{q}=\gamma \int_{\text {pulse }} \mathbf{G}\left(t^{\prime}\right) d t^{\prime}
$$

The exact value of $\mathbf{q}$ depends on the shape of the pulse profile used in the experiment. Its magnitude would be $\gamma \delta G$ for a rectangular gradient pulse of width $\delta$, but this is not feasible because the actual 


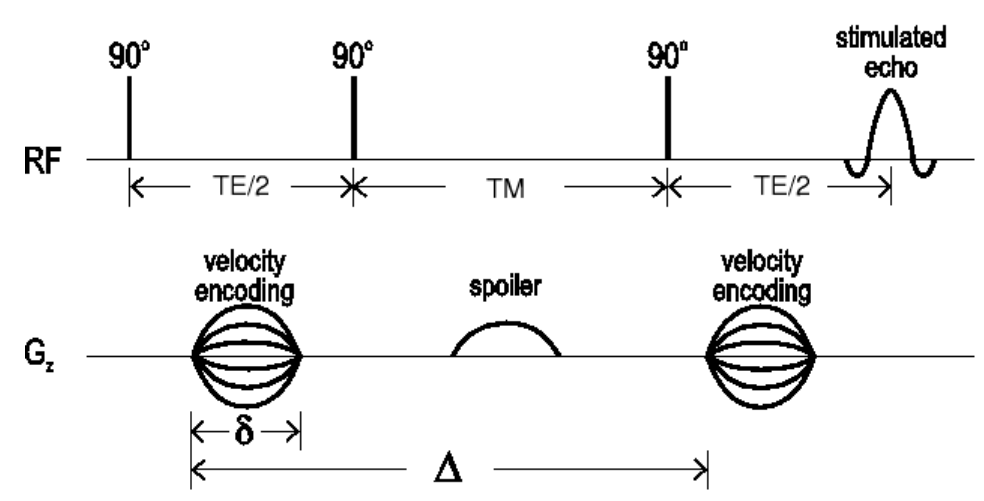

Figure 18: An example of stimulated-echo pulse sequence for detecting spin displacements along $z$-direction. The time between the second and third RF pulses, TM, is referred to as mixing time.

gradient pulse has smooth changes of the gradient field. The most commonly used gradient pulse has half-sine or trapezoidal shape.

The PFGSTE pulse sequence is shown in Fig. 18. Here, the dephasing and rephasing gradient pulses are in opposite polarity so that the phase shift is convoluted with the spin displacement. The first radio-frequency $(\mathrm{RF}) 90^{\circ}$ pulse imparts a phase shift $\mathbf{q} \cdot \mathbf{r}_{0}$ to a spin located at $\mathbf{r}_{0}$. This phase shift is inverted by the last two $90^{\circ}$ pulses in the sequence. Suppose the spin has moved to position $\mathbf{r}_{0}+\mathbf{R}$ during the time between the two velocity encoding gradients pulses, $\Delta$; then the net phase shift for that particular spin is $\mathbf{q} \cdot \mathbf{R}$. The observation time, $\Delta$, is controlled by the mixing time, TM, which is the time between the second and third RF pulses. The sequence is repeated using regularly spaced increments in the pulsed gradient field to completely observe the sample in "q-space". The spoiler gradient between the second and the third RF pulses reduces the undesired signal in the transverse evolution periods. We also use four steps of phase cycling to eliminate unwanted signals. A remarkable feature of the stimulated echo as opposed to the spin echo is that the spins display longitudinal relaxation between the last two RF pulses, which is longer than that of transverse relaxation. The stimulated echo is useful for porous media where the flow rate is relatively small, but sufficient displacement is required to observe the motion.

The spatial dependence of the molecular translations is resolved by combining the velocity imaging sequence with the conventional spatial imaging encodings. In this pulse sequence, the phase shift of spins reflects the information about their displacement as well as the spatial position. Therefore, it is important to carefully design the pulse sequence such that the desired information can be suitably deconvoluted from the measured data. In particular, it should be noted that the con- 
ventional spatial encodings, which were originally designed for a stationary fluid, may not correctly work for moving fluids due to artifacts arising from the motion. In our work, we implemented "flow compensation" in the spatial encoding gradient. Here, a compensational gradient field is added to the dephasing frequency encoding gradient and each of the velocity encoding gradients so that the effects of the fluid flow are canceled out (see Appendix 4 for more details).

We define the joint spin-displacement density function, $\varrho(\mathbf{r}, \mathbf{R})$, such that the density of spinsthe number of spins divided by the voxel volume-that have displacements between $\mathbf{R}$ and $\mathbf{R}+d \mathbf{R}$ in a voxel at $\mathbf{r}$ is $\varrho(\mathbf{r}, \mathbf{R}) d \mathbf{R}$. $\varrho(\mathbf{r}, \mathbf{R})$ can be expressed in terms of local spin density $\rho(\mathbf{r})$ and the normalized displacement distribution function $P(\mathbf{r}, \mathbf{R})$ :

$$
\varrho(\mathbf{r}, \mathbf{R})=\rho(\mathbf{r}) P(\mathbf{r}, \mathbf{R}) .
$$

The integral of $\varrho(\mathbf{r}, \mathbf{R})$ with respect to $\mathbf{R}$ over its whole domain gives the spin density at the voxel, i.e.,

$$
\int \varrho(\mathbf{r}, \mathbf{R}) d \mathbf{R}=\rho(\mathbf{r})
$$

In other words,

$$
\int P(\mathbf{r}, \mathbf{R}) d \mathbf{R}=1
$$

The observed NMR signal, $\mathrm{S}(\mathbf{k}, \mathbf{q})$, is then modulated by the two wave vectors: the first, $\mathbf{k}$, is related to the spatial density of the spins and the second, $\mathbf{q}$, is related to a spatial displacement of spins:

$$
S(\mathbf{k}, \mathbf{q})=\iint \varrho(\mathbf{r}, \mathbf{R}) \exp (i \mathbf{k} \cdot \mathbf{r}) \exp (i \mathbf{q} \cdot \mathbf{R}) d \mathbf{r} d \mathbf{R} .
$$

$\varrho(\mathbf{r}, \mathbf{R})$ can be reconstructed by taking inverse Fourier transforms on Eq. 68 with respect to each of the wave vectors:

$$
\varrho(\mathbf{r}, \mathbf{R})=\iint S(\mathbf{k}, \mathbf{q}) \exp (-i \mathbf{k} \cdot \mathbf{r}) \exp (-i \mathbf{q} \cdot \mathbf{R}) d \mathbf{k} d \mathbf{q}
$$

The joint spin-displacement density function, $\varrho(\mathbf{r}, \mathbf{R})$, and the normalized displacement distribution function, $P(\mathbf{r}, \mathbf{R})$, can be converted readily to the joint spin-velocity density function, $\varrho\left(\mathbf{r}, \mathbf{v}_{n}\right)$, and the normalized velocity distribution function, $P\left(\mathbf{r}, \mathbf{v}_{n}\right)$, respectively. Here, the net velocity $\mathbf{v}_{n}$ is defined as

$$
\mathbf{v}_{n}=\frac{\mathbf{R}}{\Delta}
$$

Once $\varrho\left(\mathbf{r}, \mathbf{v}_{n}\right)$ is obtained, the average velocity at a voxel can be easily calculated. We calculate the superficial average velocity for the use of determining permeability distributions. The superficial 
average velocity, $\mathbf{v}_{\text {avg }}^{s}(\mathbf{r})$, at a voxel at $\mathbf{r}$ is defined by

$$
\mathbf{v}_{a v g}^{s}(\mathbf{r})=\frac{1}{V_{\mathbf{r}}} \int_{V_{\mathbf{r}}} \mathbf{v}_{n}(\mathbf{s}) d \mathbf{s},
$$

where $V_{\mathbf{r}}$ denotes the volume of a voxel at $\mathbf{r}$, and $\mathbf{s}$ is another position vector indicating a position within the voxel. In Eq. 71, the term $d \mathbf{s} / V_{\mathbf{r}}$ can be interpreted as the infinitesimal volume fraction of spins that have velocity $\mathbf{v}_{n}(\mathbf{s})$. By the definition of the joint spin-velocity density function, $\varrho\left(\mathbf{r}, \mathbf{v}_{n}\right) d \mathbf{v}_{n}$ indicates the number of spins divided by the voxel volume, of which velocities are in the infinitesimal velocity range. Then, its corresponding volume fraction can be written as $\varrho\left(\mathbf{r}, \mathbf{v}_{n}\right) / \rho_{b} d \mathbf{v}_{n}$, where $\rho_{b}$ is the spin density of bulk fluid. Therefore, the superficial velocity can be written as

$$
\mathbf{v}_{\text {avg }}^{s}(\mathbf{r})=\int_{V_{\mathbf{r}}} \mathbf{v}_{n} \frac{d \mathbf{s}}{V_{\mathbf{r}}}=\int_{-\infty}^{\infty} \mathbf{v}_{n} \frac{\varrho\left(\mathbf{r}, \mathbf{v}_{n}\right)}{\rho_{b}} d \mathbf{v}_{n}=\frac{\rho(\mathbf{r})}{\rho_{b}} \int_{-\infty}^{\infty} \mathbf{v}_{n} P\left(\mathbf{r}, \mathbf{v}_{n}\right) d \mathbf{v}_{n}
$$

On the other hand, the intrinsic average velocity, $\mathbf{v}_{\text {avg }}^{i}(\mathbf{r})$, at a voxel at $\mathbf{r}$ is defined by

$$
\mathbf{v}_{a v g}^{i}(\mathbf{r})=\frac{1}{V_{\mathbf{r}}^{p}} \int_{V_{\mathbf{r}}} \mathbf{v}_{n}(\mathbf{s}) d \mathbf{s},
$$

where $V_{\mathbf{r}}^{p}$ is the pore volume in the voxel. Given that $V_{\mathbf{r}}^{p} / V_{\mathbf{r}}=\rho(\mathbf{r}) / \rho_{b}$, the combination of Eqs. (71), (72) and (73) gives

$$
\mathbf{v}_{\text {avg }}^{i}(\mathbf{r})=\int_{-\infty}^{\infty} \mathbf{v}_{n} P\left(\mathbf{r}, \mathbf{v}_{n}\right) d \mathbf{v}_{n}
$$

Practically, the quantitative superficial velocity value cannot be directly evaluated only from velocity imaging data. This is because the spin densities are not known, and what we detect from NMR experiment is not actually $\varrho\left(\mathbf{r}, \mathbf{v}_{n}\right)$ but the magnetization intensity proportional to $\varrho\left(\mathbf{r}, \mathbf{v}_{n}\right)$. In our work, we first calculate intrinsic velocity from $\varrho\left(\mathbf{r}, \mathbf{v}_{n}\right)$ using

$$
\mathbf{v}_{a v g}^{i}(\mathbf{r})=\frac{\rho(\mathbf{r}) \int \mathbf{v}_{n} P\left(\mathbf{r}, \mathbf{v}_{n}\right) d \mathbf{v}_{n}}{\rho(\mathbf{r})}=\frac{\int \mathbf{v}_{n} \varrho\left(\mathbf{r}, \mathbf{v}_{n}\right) d \mathbf{v}_{n}}{\int \varrho\left(\mathbf{r}, \mathbf{v}_{n}\right) d \mathbf{v}_{n}} .
$$

Then, the superficial velocity is calculated using porosity distribution data, $\phi(\mathbf{r})$ :

$$
\mathbf{v}_{a v g}^{s}(\mathbf{r})=\frac{V_{\mathbf{r}}^{p}}{V_{\mathbf{r}}} \mathbf{v}_{\text {avg }}^{i}(\mathbf{r})=\phi(\mathbf{r}) \mathbf{v}_{\text {avg }}^{i}(\mathbf{r})
$$

\subsubsection{Experiments}

We demonstrate the results of velocity imaging experiments for two kinds of samples. For the first, we use a relatively thin sample and resolve only two in-plane spatial coordinates $\left(z_{1}\right.$ and $\left.z_{2}\right)$. The other experiment is for the cylindrical samples presented in Table 2 which are resolved in all three coordinates. 


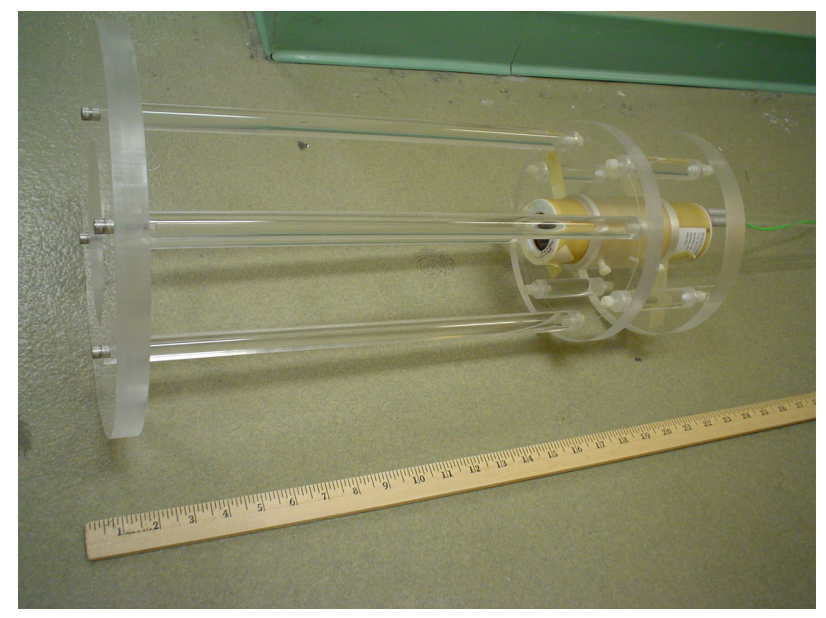

Figure 19: The "positioner" in which the RF coil is mounted.

The periphery of each sample was sealed with epoxy and mounted in a plexiglass holder with Orings or plastic gaskets and tube fittings to provide appropriate boundary conditions. The sample was initially saturated with fluid under vacuum and mounted in the RF coil. A plexiglass holder was designed to fit within the inner space of the RF coil. It was ensured that there were no air bubbles inside the sample or tube lines. A stable fluid flow through the sample along the $z_{1}$ direction was maintained using a Quizix ${ }^{\circledR}$ QL-700 pump. The pressure drop between the inlet and outlet ports was monitored by an electronic differential pressure transducer.

The position of the sample must be the same as that used for porosity imaging in order to use the porosity distribution together with the velocity imaging data. We designed a "positioner" to meet this condition (Fig. 19). The positioner is made of plexiglass, and it is designed to consistently position the RF coil and the sample inside the NMR bore.

The pulse sequence for velocity imaging was designed to have a pair of velocity encodings as described in Section 6.2.1 (Fig. 18) and spatial imaging encodings. We typically use 32 velocity encoding gradient steps. Their strengths were calculated corresponding to the velocity field of view and the length of the gradient pulse. The spatial information was frequency-encoded in $z_{1^{-}}$ direction, which is parallel to the static magnetic field $B_{0}$, and phase-encoded in the other two directions $\left(z_{2}\right.$ and $\left.z_{3}\right)$. Each pulse sequence provides the measurements of the component of the velocity vector corresponding to the direction of the velocity encoding gradient. Thus, the pulse sequence is repeated with different directions of the velocity encoding gradient to measure each component of the velocity vector. The detailed parameters for the velocity imaging experiments 


\begin{tabular}{||c|c|c||}
\hline Sample & BENT2 & BENT1 and reservoir samples \\
\hline \hline Spectral width & $30000 \mathrm{~Hz}$ & $50000 \mathrm{~Hz}$ \\
\hline Defocusing pulse width & $14 \mu \mathrm{s}$ & $50 \mu \mathrm{s}$ \\
\hline Refocusing pulse width & $28 \mu \mathrm{s}$ & $50 \mu \mathrm{s}$ \\
\hline Gradient pulse shape & sinusoidal & $2 \mathrm{~ms}$ \\
\hline Velocity encoding pulse width, $\delta$ & $2 \mathrm{~ms}$ & 32 \\
\hline Number of velocity encoding steps & 32 & $2.5 \mathrm{~mm} / \mathrm{s}$ \\
\hline Velocity field of view & $11 \mathrm{~mm} / \mathrm{s}$ & $32 \times 8 \times 8$ \\
\hline Spatial field of view & $100 \mathrm{~mm} \times 35 \mathrm{~mm}$ & $30 \mathrm{~mm} \times 30 \mathrm{~mm} \times 30 \mathrm{~mm}$ \\
\hline Data acquisition size & $128 \times 32$ & $1 \mathrm{~s}$ \\
\hline Observation time, $\Delta$ & $200 \mathrm{~ms}$ & \\
\hline Number of averages & 8 & $5 \mathrm{~s}$ \\
\hline Repetition time, $T R$ & & \\
\hline \hline
\end{tabular}

Table 8: Pulse sequence parameters used in velocity imaging

are provided in Table 8 .

For the relatively thin sample, a Bentheimer sandstone was prepared to be a rectangular parallelepiped shape having a length of $50 \mathrm{~mm}$ extending in the $z_{1}$-direction, width $25 \mathrm{~mm}$ along the $z_{2}$-direction, and thickness $5 \mathrm{~mm}$ in the $z_{3}$-direction. This sample is referred to as BENT2. NMR velocity imaging was carried out using the proton resonance signal from brine $(3 \% \mathrm{NaCl}$ and $0.03 \%$ $\mathrm{NaN}_{3}$ by weight in distilled water). The measured total flow rate was $1.5 \mathrm{ml} / \mathrm{min}$ and the pressure drop was $0.1 \mathrm{~atm}$. The apparent average velocity over the entire sample is then calculated by

$$
v^{a p p}=\frac{1.5 \mathrm{~cm}^{3} / \mathrm{min}}{2.5 \mathrm{~cm} \times 0.5 \mathrm{~cm} \times 60 \mathrm{~s} / \mathrm{min}}=0.02 \mathrm{~cm} / \mathrm{s}
$$

The viscosity of the water was estimated to be $1.0 \mathrm{cP}$ at its temperature $\left(18^{\circ} \mathrm{C}\right)$. The apparent permeability, $k^{a p p}$, of the sample can be calculated in the conventional way (see Eq. 7:

$$
k^{a p p}=\frac{0.02 \mathrm{~cm} / \mathrm{s} \times 1.0 \mathrm{cP}}{0.1 \mathrm{~atm} / 5.0 \mathrm{~cm}}=1.0 \text { Darcy. }
$$

The NMR experiment was performed using a General Electric 2T Omega CSI system with an Oxford Instruments superconducting magnet having 31-cm-diameter horizontal bore. A 4.4-cmi.d. birdcage RF resonator was used for RF transmission and reception of the NMR signal. 


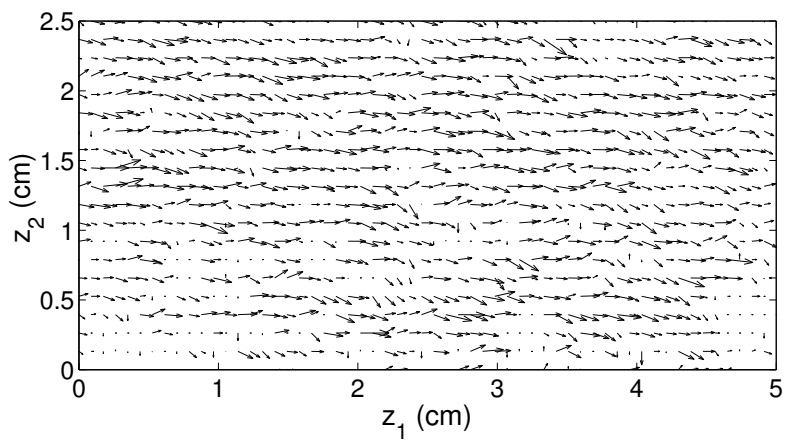

Figure 20: Superficial average velocity of the thin Bentheimer sample (BENT2)

The experiment with the cylindrical samples (BENT1 and reservoir samples) was performed with the Bruker BioSpec ${ }^{\circledR}$ 24/30 system. The velocity encoding pulse sequence was implemented in the ParaVision ${ }^{\circledR}$ pulse programming system. We used de-ionized water for this experiment. Glass beads of $0.1-\mathrm{mm}$ diameter were inserted at the entrance face of the sample to let the entering flow distribute uniformly at the entrance surface. The pressure drop across the Bentheimer sample was $0.127 \mathrm{~atm}$, and the volumetric flow rate was controlled to be $20 \mathrm{ml} / \mathrm{min}$. The apparent permeability calculated by Eq. 78 is 0.267 Darcy.

\subsubsection{Results and discussion}

Figure 20 shows the measured superficial average velocity of the thin Bentheimer sample (BENT2) using $58 \times 20$ image voxels. Each arrow represents the direction and magnitude of the superficial average velocity at the corresponding voxel. The irregular directions of the fluid flow reflect heterogeneities of the permeability.

Note that the velocity vector presented in Fig. 20 is not actually raw data but calculated from the corresponding spin-velocity density function, $\varrho\left(\mathbf{r}, \mathbf{v}_{n}\right)$ (see Eqs. 69-76). The function $\varrho$ with respect to $\mathbf{v}_{n}$ is represented by 32 discrete numbers according to the number of velocity encoding steps. We interpolate these points to represent the continuous density function. Figure 21 shows an example of the joint spin-velocity density function of a voxel evaluated from the cylindrical Bentheimer sample (BENT1). This figure indicates that the $z_{1}$-component of velocity vector varies from $0 \mathrm{~mm} / \mathrm{s}$ to $+1.5 \mathrm{~mm} / \mathrm{s}$ in the voxel.

The superficial average velocities measured for BENT1 are presented in Fig. 22. The figure shows that the fluid flows through the media in the positive $z_{1}$-direction on average and that local 


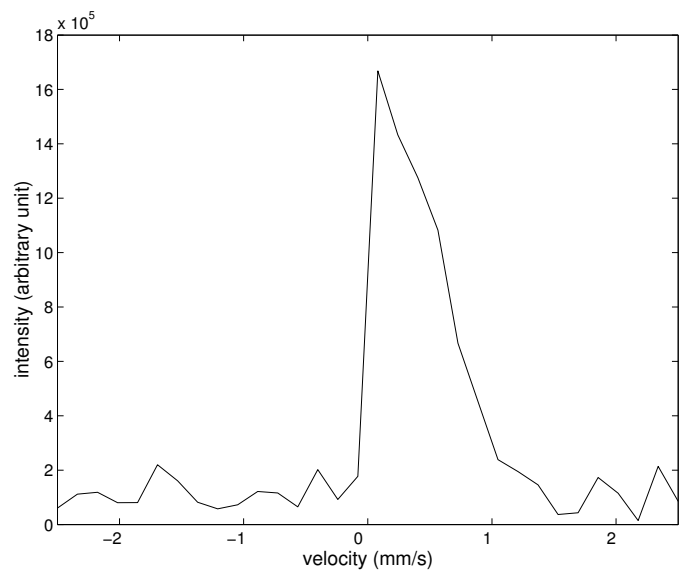

Figure 21: An example of joint spin-velocity density function $\left(z_{1}\right.$-component, the coordinate numbers for the voxel are $Z_{1}=18, Z_{2}=3$ and $Z_{3}=6$ )

heterogeneities in the sample cause variations in the velocity field.

It should be noted that not all the voxels within the spatial field of view correspond to the sample, since the size of the sample is smaller than the field of view. For the three-dimensional velocity imaging, we determined that the sample is located within $6 \leq Z_{1} \leq 27,1 \leq Z_{2} \leq 7$, and $1 \leq Z_{3} \leq 7$ out of $32 \times 8 \times 8$ voxels. There are still more voxels within this range that do not correspond to the sample, due to the cylindrical shape of the sample. Some of them are located completely outside of the sample, while others cross the boundaries of the sample for which only a portion of the voxel volume correspond to the sample.

As described in detail in Section 6.3, we utilize measured velocities and the corresponding quantities computed using the mathematical model of the experiment. Those computations are done using a finite difference solution with Cartesian spatial coordinates. A difficulty is encountered in analyzing three-dimensional velocity imaging due to the use of a Cartesian coordinate system for the representation of voxels and for the finite difference grid, while the sample has a cylindrical shape. Consequently, the boundaries of the sample often appears within a voxel, but it is not easy to exactly locate them. Furthermore, the boundary conditions along the curved surfaces cannot be accurately handled. In this work, we deal with a virtual sample when we analyze velocity imaging on cylindrically shaped sample. The virtual sample is a parallelepiped and represented by the voxels at $6 \leq Z_{1} \leq 27,1 \leq Z_{2} \leq 7$, and $1 \leq Z_{3} \leq 7$. Here, the actual sample is considered as a part of the virtual sample. We have porosity values of zero for the voxels completely outside of the actual sample, which results in values of zero for the superficial velocity. For the voxels crossing the 


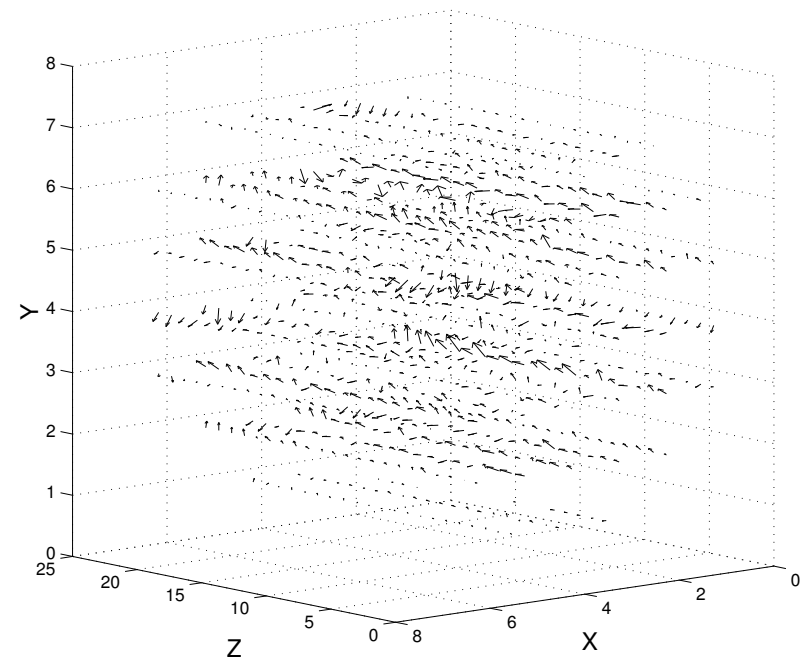

Figure 22: Superficial average velocity of the cylindrical Bentheimer sample

boundaries of the actual sample, the superficial velocity averaged over the voxel volume (Eq. 71) is calculated. As will be shown in Section 6.3, this can be a good approximation when the voxel size is sufficiently smaller than the sample size.

The velocity imaging experiments were also performed on the reservoir samples. However, it was found that the intensity of the stimulated echo for those samples is not significant enough to provide reliable estimates of the velocity distributions. We suppose the most important reason for the problem is the relatively strong magnetic susceptibility of the reservoir samples, which leads to intensive internal magnetic field gradients. Moreover, the significant motion of the fluid makes the spins experience more inhomogeneous magnetic fields in velocity imaging experiments. The transverse relaxation consequently tends to be fast and meaningful signals may not be left in the detected stimulated echo. Note NMR porosity imaging worked with the reservoir samples (Section 5), but that experiment differs from velocity imaging in that: 1. the fluid is stationary, 2. the echo time is much shorter than that of PFGSTE sequence, 3. there is no mixing time, and 4. there is no velocity encoding gradient which may cause internal magnetic field gradients.

We estimated the typical attenuation of the magnetization to evaluate the signal intensity of the detected stimulated echo. The typical attenuation of the magnetization is represented in terms of the average relaxation times by

$$
\exp \left(-T E / T_{2}^{a v g}\right) \times \exp \left(-T M / T_{1}^{a v g}\right)
$$




\begin{tabular}{||c||c||}
\hline \hline Sample & $\exp \left(-T E / T_{2}^{a v g}\right) \times \exp \left(-T M / T_{1}^{a v g}\right)$ \\
\hline \hline R138 & 0.0418 \\
\hline R145 & 0.0208 \\
\hline R221 & 0.0443 \\
\hline R237 & 0.0973 \\
\hline BENT1 & 0.3718 \\
\hline \hline
\end{tabular}

Table 9: Typical attenuation of the samples $(T E=7.5 \mathrm{~ms}$ and $T M=200 \mathrm{~ms})$

$T M$ is the mixing time that represents the time between the second and third RF pulses. Using the average relaxation times presented in Table 3, the typical values for the echo time, $T E=7.5 \mathrm{~ms}$, and the mixing time, $T M=200 \mathrm{~ms}$, typical attenuation is calculated for each sample (Table 9 ). The table shows that the characteristic relaxation times of the reservoir samples are significantly shorter than those of the Bentheimer sample.

We present some examples of velocity imaging experiments on the Bentheimer and the R237 samples to illustrate the discussions above. The one-dimensional velocity imaging experiments were performed on each of the samples with stationary $(0 \mathrm{ml} / \mathrm{min})$ and flowing $(20 \mathrm{ml} / \mathrm{min})$ fluid. Figure 23 shows the results from the Benheimer sample. It presents the detected stimulated echo intensity ( $\mathrm{a}$ and $\mathrm{c}$ ) and their corresponding inverse Fourier transformation, i.e., the joint spinvelocity density function ( $b$ and $d$ ) for the two flow rates. If the phase shift induced by the dephasing gradient is completely reversible by the rephasing gradient, the intensity of the stimulated echo would be a constant regardless of the velocity encoding gradient strengths. This would result in the representation of the joint spin-velocity density function as a Dirac delta function. However, the net phase shift after the dephasing and rephasing gradients can hardly be zero for most of the experiments. For a flowing fluid, the effect of flowing motion is dominant on the net phase shift so that it can be used to measure the molecular displacement. The net phase shift is still nonzero for a stationary fluid due to the self-diffusion arising from Brownian motion. Even for a solid, the net phase shift tends not to be zero because internal magnetic field gradients arise when the pulsed gradient fields are applied. Figure 23(a) shows that the stimulated echo intensity decreases as the absolute value of the velocity encoding gradient (i.e., the wave vector $\mathbf{q}$ ) increases for the stationary fluid. Consequently, the joint spin-velocity density function is spread out from a Dirac delta function (Fig. 23b). We estimate that the density function is broader than it would 
be with only self-diffusion due to the effect of internal magnetic field gradients. The results from flowing fluid (Figs. $23 \mathrm{c}$ and d) shows that the joint spin-velocity density function is shifted to the positive side of the velocity axis when fluid flows. On the other hand, the similar velocity imaging experiments performed on the R237 sample (Fig. 24) show that the stimulated echo with respect to the velocity encoding gradients is not significant enough to resolve the velocities.

We have looked into modifying pulse sequences to improve the signal intensity with reservoir samples. We implemented alternating gradients to eliminate the effect of internal magnetic field as we do in our diffusion experiment (Section 4.4). However, the critical problem in this sequence is that the echo time is further extended due to additional gradient and RF pulses. It turns out that the intensity of the stimulated echo was not much improved. We note again that the stimulated echo intensity of the moving fluid is smaller than that of the stationary fluid because the moving fluid experiences a more inhomogeneous magnetic field. Also, the mixing time for velocity imaging is generally longer than that of the diffusion experiment. The alternating gradient sequence may work for the diffusion experiment with stationary fluid, but it was not effective for the velocity imaging experiment on those reservoir samples.

It may be possible to determine the velocity distributions for these challenging samples if a fluid can be identified whose relaxation rate is not so negatively affected by the solid surfaces, as is water. We have tried oil (Soltrol ${ }^{\circledR}$ 130, Phillips Petroleum Co.), and found that intensity of the stimulated echo can be improved. However, it was not sufficient for determination of the velocity distributions. Hyperpolarized gases, such as xenon, may be viable, although significant additional equipment and changes in imaging protocols would be required. At any rate, the method will likely be viable with many carbonate samples since saturating water tends to exhibit smaller relaxation rates than with sandstones. 


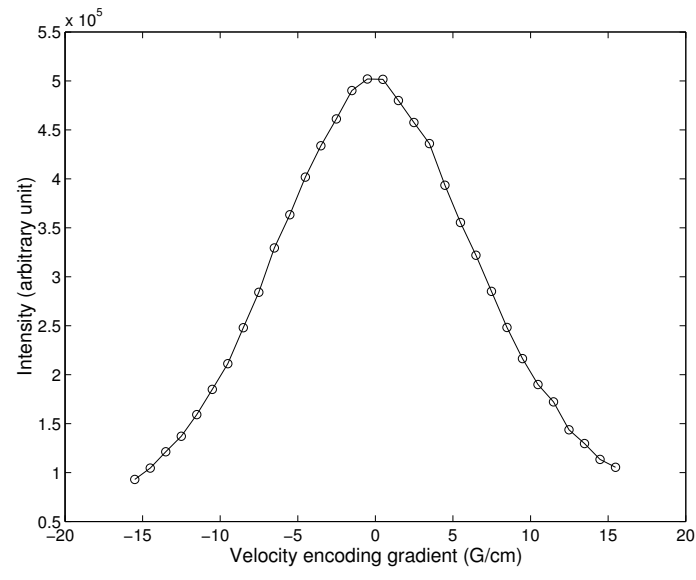

(a)

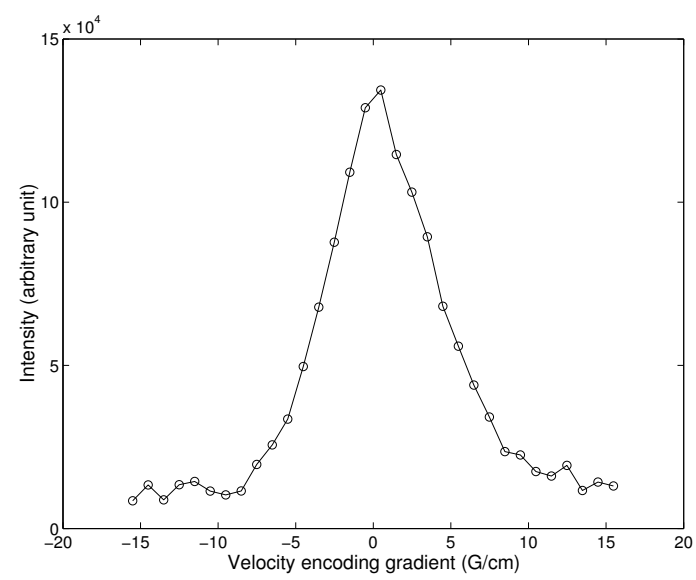

(c)

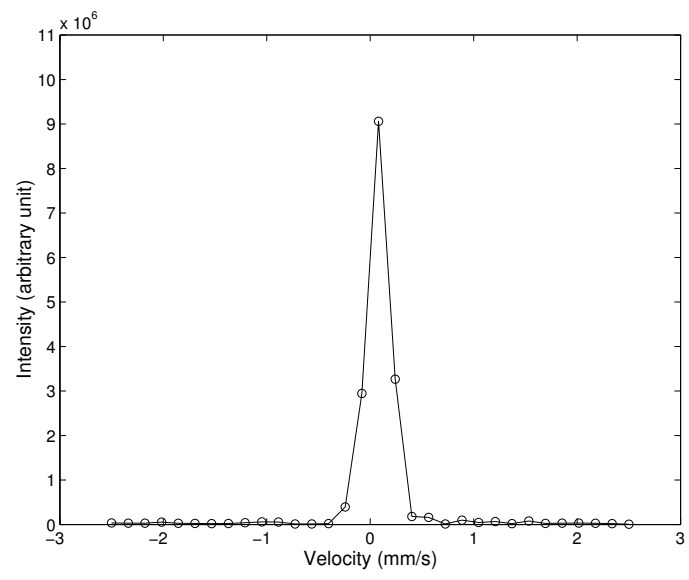

(b)

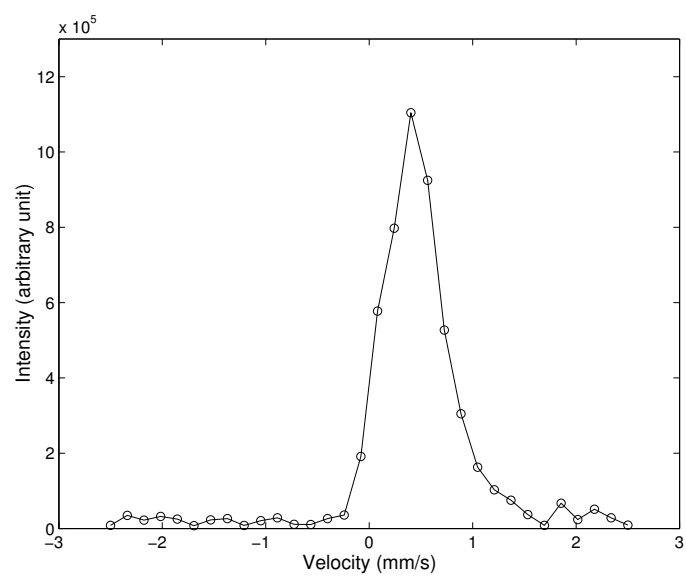

(d)

Figure 23: Velocity encoded stimulated echo data (a,c) and their joint spin-velocity density function (b,d) of a voxel in the Bentheimer sample with flow rates $0 \mathrm{ml} / \mathrm{min}(\mathrm{a}, \mathrm{b})$ and $20 \mathrm{ml} / \mathrm{min}(\mathrm{c}, \mathrm{d})$ 


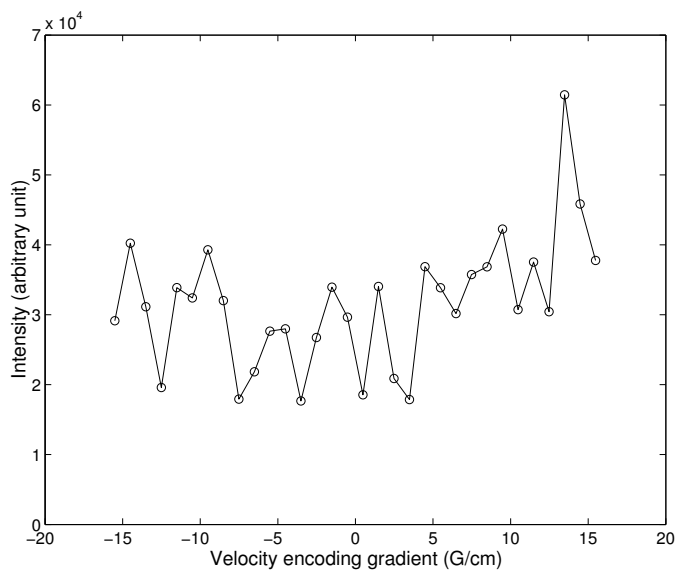

(a)

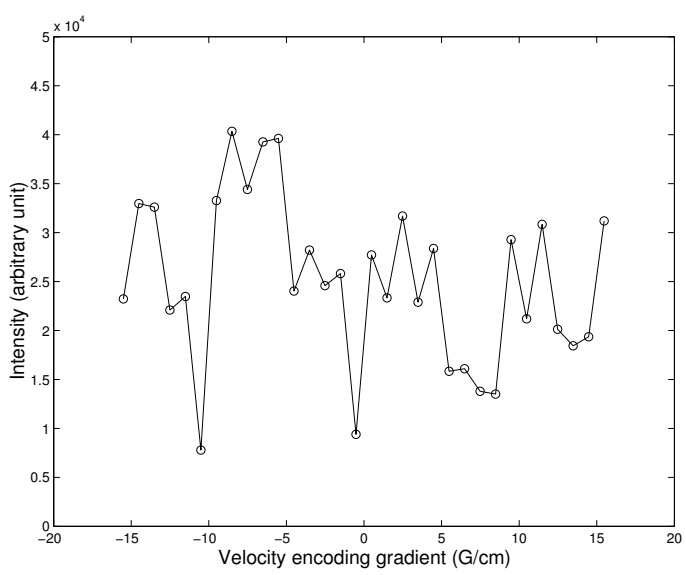

(c)

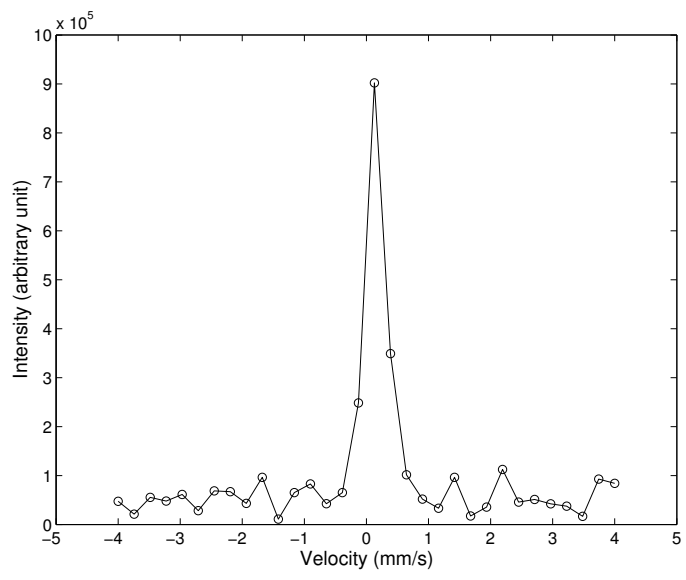

(b)

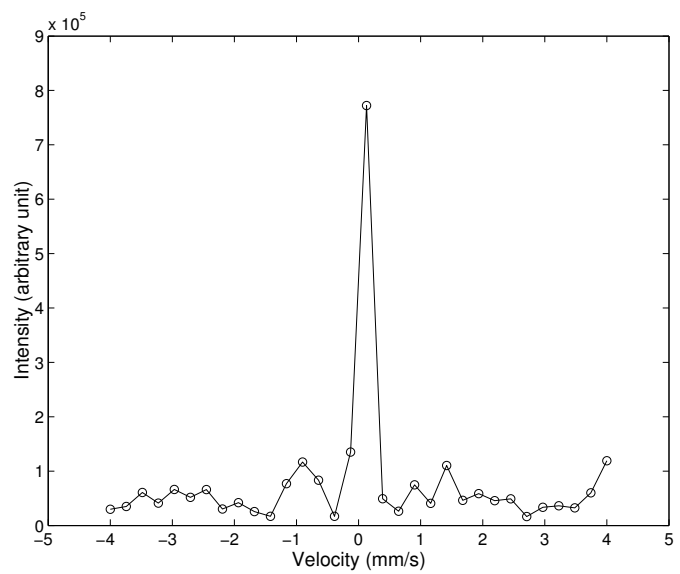

(d)

Figure 24: Velocity encoded stimulated echo data (a,c) and their joint spin-velocity density function (b,d) of a voxel in $\mathrm{R} 237$ sample with flow rates $0 \mathrm{ml} / \mathrm{min}(\mathrm{a}, \mathrm{b})$ and $20 \mathrm{ml} / \mathrm{min}(\mathrm{c}, \mathrm{d})$ 


\subsection{Determination of permeability distributions}

\subsubsection{Methodology}

The permeability can be determined using the measured velocity distribution. However, explicit calculation of the permeability is not possible (note, for example, that the pressure gradient in the Darcy equation (Eq. 2) is not known). The permeability distribution can be determined from solution of an inverse problem. Our work here is based on the assumption that the permeability is isotropic, although our work can be extended to the more general case.

The goal of the inverse problem is to find a permeability distribution that minimizes the discrepancy between the observed experimental data and that calculated through the solution of the governing equations. The problem can be expressed formally as a minimization problem:

$$
\min _{k} J=J_{\text {expt }}+\lambda J_{\text {reg }}
$$

Here, $J$ is the objective or cost function, which consists of two terms. The first, $J_{\text {expt }}$, measures the difference between experimentally observed and calculated values of the data, while the second, $J_{\text {reg }}$, the regularization term, is used to impose any a priori knowledge of the permeability distribution onto the solution. This knowledge can appear in the form of smoothness constraints, which is used in this work, or in the form of actual permeability measurements at specified locations. These terms take the following forms:

$$
\begin{aligned}
J_{\text {expt }}= & \frac{1}{2} \sum_{n}^{N_{\mathbf{v}}} \sum_{i d}^{3} \int_{V} \mathrm{w}_{v_{z_{i d},(n)}}\left(v_{z_{i d},(n)}^{o b s}-v_{z_{i d},(n)}^{c a l}\right)^{2} \hat{\delta}\left(V, V_{(n)}\right) d V \\
& +\sum_{n}^{N_{p}} \frac{1}{2} \int_{V} \mathrm{w}_{p_{(n)}}\left(p_{(n)}^{o b s}-p_{(n)}^{c a l}\right)^{2} \hat{\delta}\left(V, V_{(n)}\right) d V \\
J_{r e g}= & \sum_{i d=1}^{3} \int_{V}\left(\frac{d^{d o} k}{d z_{i d}^{d o}}\right)^{2} d V \quad d o \in\{1,2,3, \ldots\}
\end{aligned}
$$

The Kronecker delta function is defined as

$$
\hat{\delta}\left(V, V_{(n)}\right)=\left\{\begin{array}{ll}
1 & V=V_{(n)} \\
0 & \text { otherwise }
\end{array} \quad \text { or } \quad \hat{\delta}\left(\mathbf{z}, \mathbf{z}_{(n)}\right)= \begin{cases}1 & \mathbf{z}=\mathbf{z}_{(n)} \\
0 & \text { otherwise }\end{cases}\right.
$$

where $\mathbf{z} \in V$ and $\mathbf{z}_{(n)} \in V_{(n)}$, and $V_{(n)}$ represent the points at which the superficial average velocity or pressure are measured. Here, $\mathbf{v}^{o b s}$, and $p^{o b s}$ are observed experimental data and $\mathbf{v}^{c a l}$, and $p^{c a l}$ represent the corresponding calculated values from the numerical simulation. In the experimental data fit term, the differences between observed and calculated properties are individually weighted 


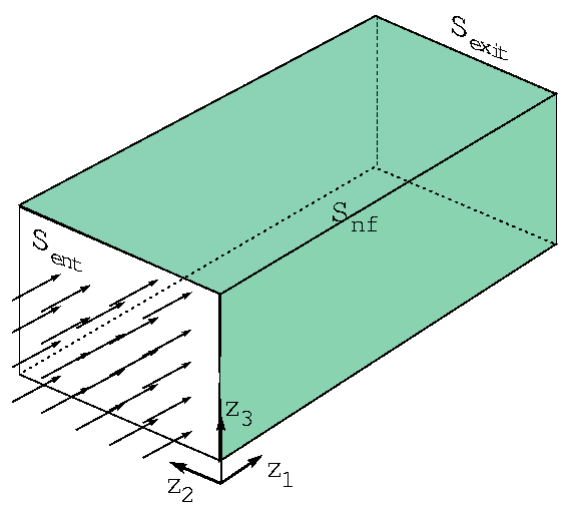

Figure 25: The domain of the fluid flowing system

by $\mathrm{w}_{v_{z_{i d}},(n)}$, and $\mathrm{w}_{p_{(n)}}$. These values depend on the relative accuracy of the experimental data, and are often taken to be the inverse of the variance of the errors in the experimental measurements. In our work, we assume that the errors have mean zero and are identically distributed, so that the weight value is unity. The regularization term is weighted relative to the data fitting term by the regularization parameter, $\lambda$, which must be suitably determined. Currently, we are determining the regularization parameter, $\lambda$, by examining the degree of fit, $J_{\text {expt }}$, using several different values for $\lambda$, and estimating the maximum value of $\lambda$ which does not compromise the fit to the data (Yang and Watson 1988).

The calculated values required to evaluate the objective function are determined by solving the locally volume-averaged equation of continuity and the differential momentum balance (Eqs. 1 and 2) with appropriate boundary conditions. This is known as solving the forward problem. For a steady-state incompressible fluid phase in porous media, the continuity equation (see Eq. 1 becomes:

$$
\begin{gathered}
\nabla \cdot \mathbf{v}=0 \\
\frac{k}{\mu} \nabla p+\mathbf{v}=0
\end{gathered}
$$

Using the Darcy equation (Eq. 2), we can eliminate the velocity to obtain:

$$
\nabla \cdot\left[\frac{k}{\mu} \cdot(\nabla p-\rho \mathbf{g})\right]=0
$$

In this work, we deal with a rectangular parallelepiped domain for the fluid flowing system (Fig. 25). The dimension of the domain is represented by $L_{1}, L_{2}$, and $L_{3}$. A fluid is introduced 
at the entrance face $S_{\text {ent }}$, located at $z_{1}=0$, at a constant flow rate. No fluid is allowed to enter or leave the domain through the sealed periphery surface, $S_{n f}$, and all of the fluid is eventually expelled out at the exit face $S_{\text {exit }}$. The boundary conditions formally take the following forms:

$$
\begin{array}{cc}
p\left(0, z_{2}, z_{3}\right)=p_{\text {in }}-\rho g\left(z_{3}-z_{3}^{\text {in }}\right) & \\
p\left(z_{1}, z_{2}, L_{3}\right)=p_{\text {out }}-\rho g\left(z_{3}-z_{3}^{\text {out }}\right) & \\
\mathbf{v}\left(z_{1}, z_{2}, z_{3}\right) \cdot \mathbf{n}=0 & \text { for } z_{2}=0, L_{2}, \\
\mathbf{v}\left(z_{1}, z_{2}, z_{3}\right) \cdot \mathbf{n}=0 & \text { for } z_{3}=0, L_{3} .
\end{array}
$$

$z_{3}^{\text {in }}$ and $z_{3}^{\text {out }}$ represent reference points at which $p_{\text {in }}$ and $p_{\text {out }}$ are specified, respectively. The unit vector $\mathbf{n}$ is normal to $S_{n f}$. The gravity is represented as a scalar in understanding that its direction is opposite to $z_{3}$.

Equation 84 is solved with the boundary conditions 85 to determine the pressure distribution for a given permeability distribution. Once the pressure distribution is determined, the velocity can be computed using Eq. 2. In our current formulation, the state equation (Eq. 84) is solved by using finite differences (Seto 1999). The calculation requires the solution of large, linear systems that are iteratively solved using a conjugate gradient method with incomplete Choleski decomposition and adaptive Chebychev polynomial preconditioning (Ashby 1988). The two-tiered preconditioning scheme allows for the efficient handling of large, three-dimensional systems.

The solution of the forward problem yields pressure and velocity fields throughout the spatial domain of the system. The exact local values are dependent upon the permeability distribution used when solving the forward problem. In this work, the porous media is assumed to be isotropic and the permeability is interpreted to be a scalar function of position. Mathematically, the distribution is represented using normalized tensor product B-splines (Lee et al. 1986; Schumaker 1981):

$$
k(\mathbf{z})=\sum_{i}^{N_{1}} \sum_{j}^{N_{2}} \sum_{k}^{N_{3}} C_{i, j, k} B_{i}^{m_{1}}\left(z_{1}\right) B_{j}^{m_{2}}\left(z_{2}\right) B_{k}^{m_{3}}\left(z_{3}\right),
$$

where $m$ is the order of the $\mathrm{B}$-spline, $\mathrm{B}_{i}^{m_{1}}\left(z_{1}\right)$ is the $i$ th $\mathrm{B}$-spline basis function in the $z_{1}$ direction, and $C_{i, j, k}$ is the corresponding coefficient. The problem now involves determining the set of Bspline coefficients that minimizes Eq. 80, subject to the system equations (Eqs. 82 and 2) and the associated boundary conditions.

We are using a combination of global and local optimization methods. A robust, accurate local method is essential since sub-optimal solutions could provide significantly different estimates. We first use a global optimization method by simulated annealing (Kirkpatrick et al. 1983; Corana 
et al. 1987) to provide a good starting point for the local algorithm, since there are likely many local optima. Then, a quasi-Newton method, Broyden-Fletcher-Goldfarb-Shanno (BFGS) (Powell 1975), is used to determine the coefficients that minimize the performance index.

The BFGS method requires the derivative of the objective function with respect to the permeability. While there are several methods for calculating these gradients, the most efficient for the present case is the method of adjoint states (Chen et al. 1974; Chavent etal. 1975; Neuman 1980; Cacuci etal. 1980). The formulation of the adjoint problem uses calculus of variations to obtain first an expression for the desired derivatives in terms of an adjoint variable, $\Psi$, and second, a system of equations and boundary conditions describing $\Psi$, which are closely parallel to those found for pressure, $p$, in the forward problem. The adjoint equations are derived directly from the differential equations and boundary conditions for pressure. The adjoint variable, $\Psi$, is called the adjoint state of the pressure, $p$, and the adjoint velocity, $\mathbf{v}_{\Psi}$, is defined corresponding to $\Psi$. The $\Psi$ value associated with the locations where the pressure is specified or known through boundary conditions is zero.

\subsubsection{Validation with simulated data}

The methodology of determining permeability distributions using velocity imaging is validated here. A "true" permeability distribution is specified and the velocity data corresponding to the true permeability distribution are generated by solving the forward problem. The simulated velocity data are then used to estimate the permeability distribution and it is compared with the true distribution.

We selected clay lenses for the true distribution as presented in Fig. 26. In order to present the permeability in the three-dimensional domain, the figure is composed of sixteen small pairs of pictures, each pair of which has a contour and a surface plot of permeability and corresponds to a horizontal layer of the domain. The vertical position, $\mathrm{Y}$, of the voxels in a layer is numbered from the bottom to the top. The length of the domain is $1 \mathrm{~cm} \times 1 \mathrm{~cm} \times 1 \mathrm{~cm}$. The permeability distribution is uniform ( $\mathrm{k}=0.07$ Darcy) throughout the domain except for the area of the two clay lenses where the permeability is lower ( $\mathrm{k}=0.005$ Darcy) than other places. Consequently, the simulated velocity distribution (Fig. 27) shows that the flow does not easily pass through the clay lenses.

Using the simulated velocities, the permeability distribution is reconstructed by solving the inverse problem. The performance index term, $J_{\text {expt }}$, in Eq. 81 is set to be composed of differences 
between the simulated velocity data and their corresponding calculated values. We did not use pressure terms in the performance index. The second derivative of permeability was used $(d o=2)$ for the regularization term, $J_{r e g}$. The number of simulated velocity vector data was $16 \times 8 \times 8$. The degrees of freedom, which is the number of B-spline coefficients, of the permeability function was selected to be the same as the number of data. The finite difference grid was set as $32 \times 16 \times$ 16.

The regularization parameter, $\lambda$ is determined such that we obtain the smoothest permeability function that does not compromise the precision of the fit to the data. In order to do this, the minimization of the performance index was repeated and the root-mean-squared-error (RMSE) of velocity is calculated with respect to various $\lambda$ (Fig. 28). Here, the RMSE of velocity is defined as

$$
\operatorname{RMSE}(v)=\sqrt{\frac{1}{N_{\mathbf{v}}} \sum_{n}^{N_{\mathbf{v}}} \sum_{i d}^{3} \int_{V}\left(v_{z_{i d},(n)}^{o b s}-v_{z_{i d},(n)}^{c a l}\right)^{2} \hat{\delta}\left(V, V_{(n)}\right) d V}
$$

Then, the largest $\lambda$ that does not make a significant increase of RMSE is selected. For the example of clay lenses given in Fig. $28, \lambda$ is selected to be $3.0 \times 10^{4}$.

The estimated permeability distribution with the determined regularization parameter is presented in Fig. 29. The location and shape of the clay lenses are consistent with the true distribution. The RMSE between the true, $k^{\text {true }}$, and the estimated permeability, $k^{c a l c}$, defined as

$$
\operatorname{RMSE}(k)=\sqrt{\frac{1}{V} \int_{V}\left(k^{\text {true }}-k^{\text {calc }}\right)^{2} d V},
$$

is 0.0116 . These results show that the methodology properly works for determination of threedimensional permeability distributions. The errors of the estimation arise mostly from the limitation of the velocity data used. We would have better results in the above simulated experiment simply by increasing the resolution of the velocity data. In actual experiments, however, the resolution is inevitably limited by the equipment. In Section 6.3.4 we will discuss how we can improve the experimental designs to have more accurate estimation. 

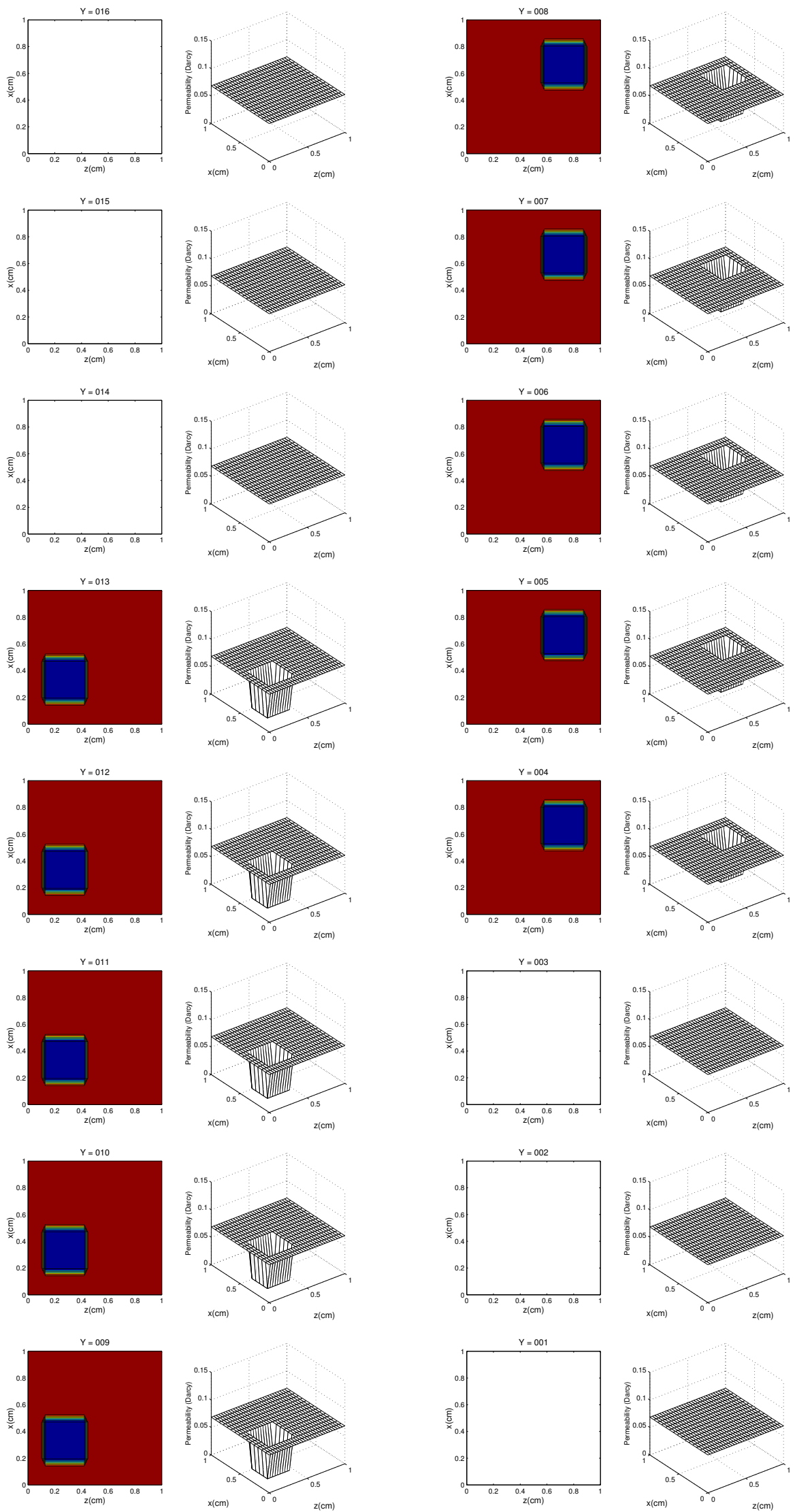

Figure 26: The "true" permeability distribution 


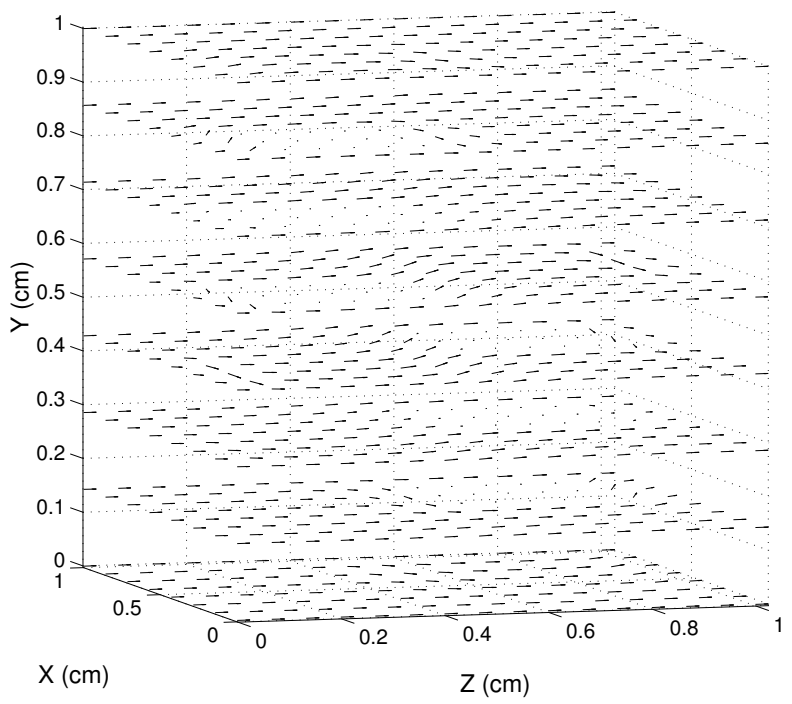

Figure 27: Simulated velocity distribution calculated with the "true permeability"

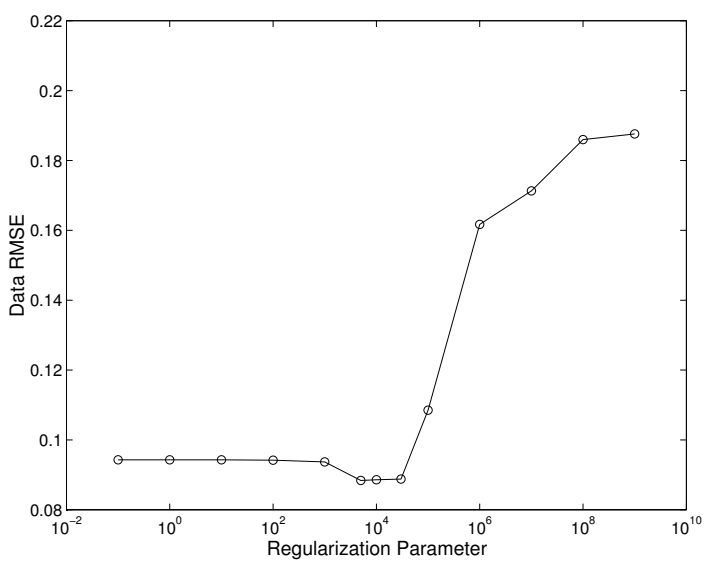

Figure 28: RMSE as a function of regularization parameter $\lambda$. 

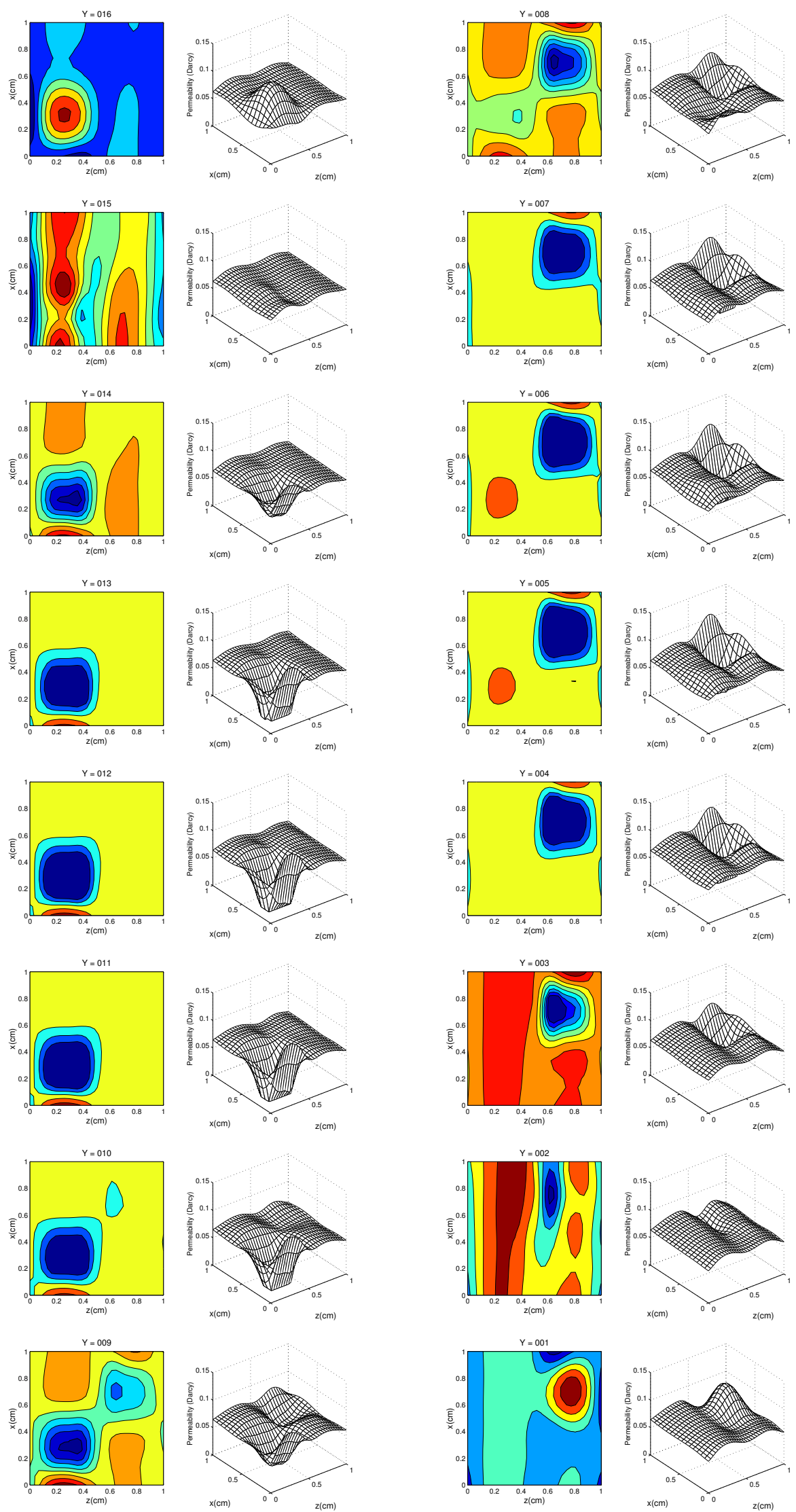

Figure 29: Permeability distribution of clay lens(1) 


\subsubsection{Analysis with experimental data}

The methodology for determining permeability distributions is now applied to the experimental velocity imaging data presented in Section 6.2. The boundary conditions presented in Eq. 85 are used, except that the condition corresponding to the $z_{3}$ coordinate was omitted for the thin sample.

The permeability distribution estimated for the thin Bentheimer sample (BENT2) is presented in Fig. 30. We used 30 equally spaced B-spline knots in each direction $z_{1}$ and $z_{2}$ to represent the permeability distribution and a finite-difference grid of $128 \times 64$. The permeability values range from 0.2 to 1.4 Darcy, indicating a fairly large variation within the sample. It can be observed that the permeability distribution (Fig. 30) is not inconsistent with the velocity data (Fig. 20), i.e., generally, the regions that have relatively small velocities tend to have smaller permeabilities than other regions.

For BENT1 and reservoir samples, the analysis was performed on the parallelepiped domain as discussed in Section 6.2; we assign the voxels outside of the sample to have velocity data which is zero. We obtained superficial average velocity vectors for $20 \times 7 \times 7$ voxels. These data were scaled to meet the apparent continuity, i.e., the flux through any cross-sectional area is to be the same. We used $12 \times 4 \times 4$ equally spaced B-spline knots and a finite difference grid of $64 \times 32 \times$ 32.

The determined permeability distribution is presented in Fig. 31 in the same way as Figs. 26 and 29. Here, the permeability is averaged for each volume element while the determined permeability distribution is a continuous function and represented by the finite difference grids. The permeability outside of the sample is determined as zero. It is seen that there are relatively large spaces with zero permeabilities at the layers near the periphery ( cylindrical shape of the sample. 

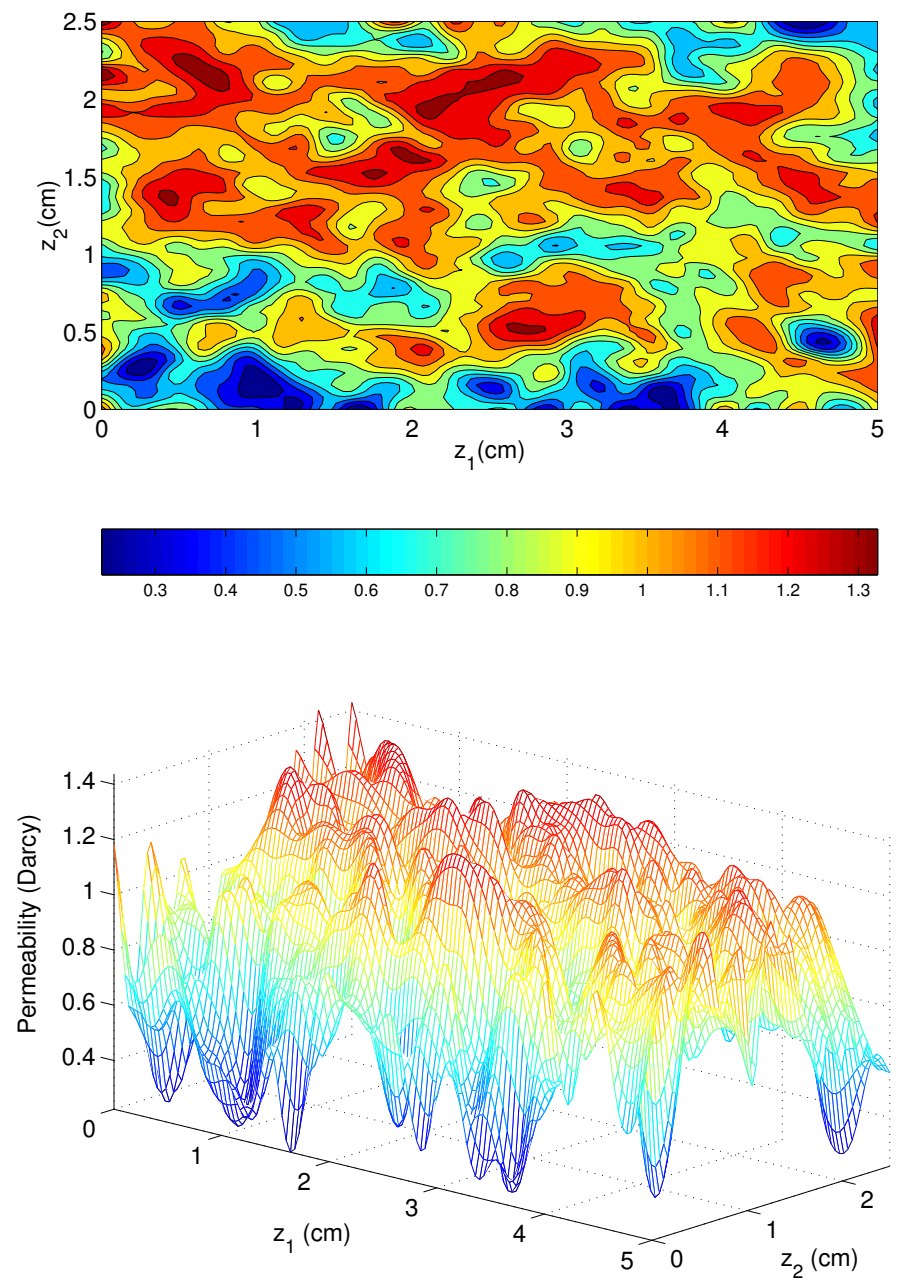

Figure 30: Determined permeability distribution using the velocity distribution in Fig. 20 

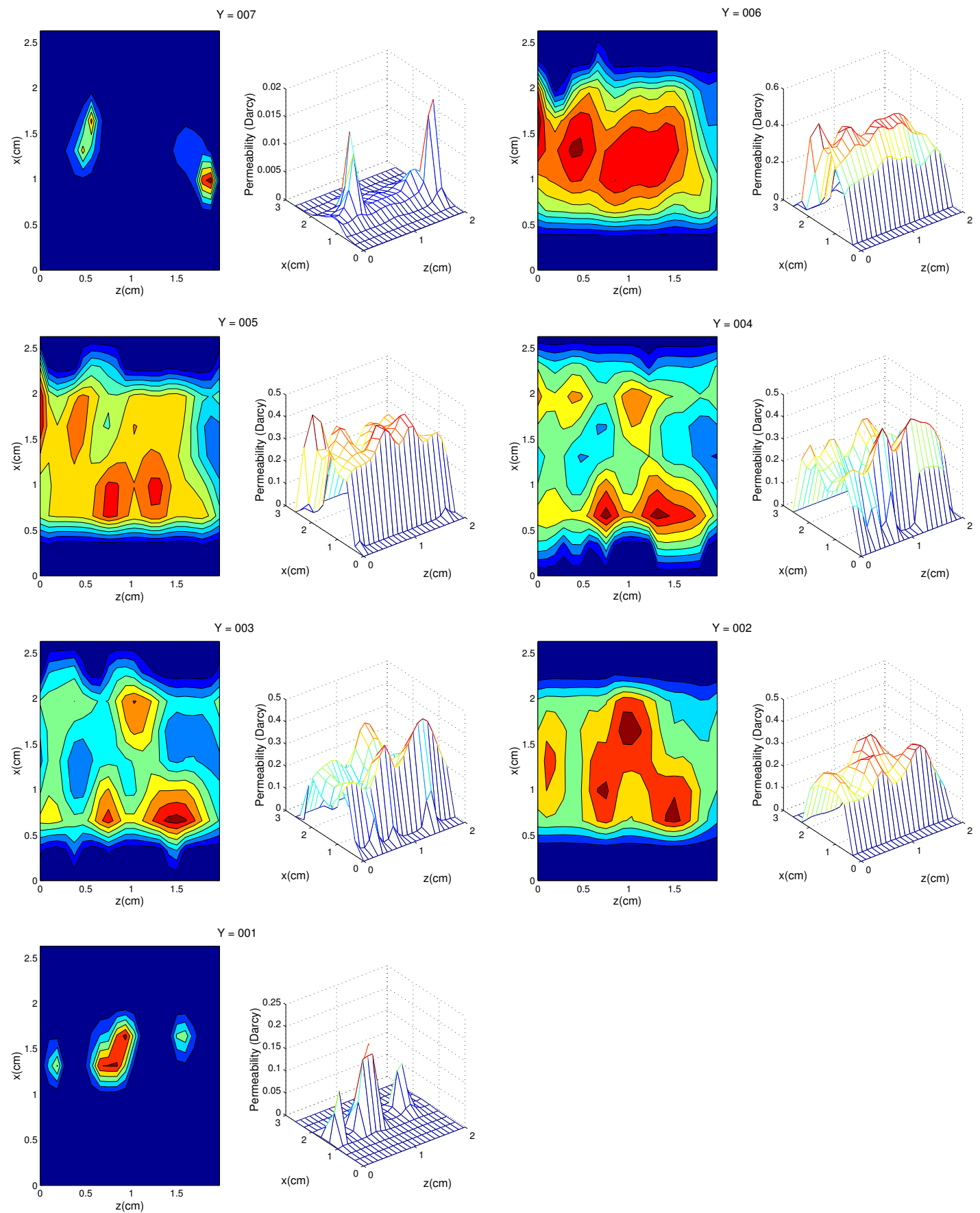

Figure 31: Determined permeability distribution using the velocity distribution in Fig. 22 


\subsubsection{Improved experimental design}

An experimental design is the protocol by which the experiment is conducted and data are measured. The experimental design can significantly affect the accuracy of the associated estimates. Our recognition that there are certain spatial configurations that, if they were present, they would not be identified (Watson et al. 2002) has led us to consider alternative experimental designs that may provide more reliable estimates of the intrinsic permeability.

There can be many possibilities of the experimental designs for the determination of permeability distributions. In general, more accurate estimates are expected as more data are added to the estimation, but the trade-offs, such as the experimental costs and convenience, must also be considered. Seto (1999) presented candidate experimental designs whereby transverse pressures on the periphery of the sample would be measured in addition to the velocity distributions. Numerical simulation showed that better estimates of permeability are obtained as more pressure data are added. However, this work has not been implemented experimentally because of experimental difficulties associated with obtaining pressure measurements around the periphery of the sample during NMR imaging experiments.

In this work, we propose new experimental designs, which are practically realizable, to improve the accuracy of the estimates of permeability distributions. The idea is that we perform multiple velocity imaging experiments on a given sample with different flow conditions and use the data from all the experiments. The multiple flow scenarios provide additional information content about the permeability distribution.

Some candidate experiments are illustrated in Fig. 32. The arrows represent injection into, or production from, the sample across the regions denoted with the thin lines. The fluid flows into and exit from the sample through the interfaces represented by thin lines. The thick lines around the sample indicate boundaries through which the fluid is not allowed to flow. Experiment A represents the conventional experiment. Experiment B is also a conventional experiment, but it is conducted by flowing fluid in a different direction. In experiments $\mathrm{C}$ and $\mathrm{D}$, the inlet and outlet faces of the sample are partially blocked so that the fluid flows in different ways. There can be many other possibilities of the flow conditions depending on the ways of preparing the boundaries

of the sample and the inlet and outlet conditions of the flow. In our new experimental design, we combine the data from several experiments. The data fitting term, $J_{\text {expt }}$ in the performance index 
(Eq. 81) associated with the new experimental design can be expressed as

$$
J_{\text {expt }}=\sum_{\Gamma} J_{\text {expt }, \Gamma}, \quad \Gamma \in\{\text { selected experiments }\} .
$$

Here, the subscript $\Gamma$ represents one of the flow experiments from among the chosen set. The observed and calculated velocities ( $v^{o b s}$ and $v^{c a l}$ in Eq. 81) associated with the data fitting term, $J_{\text {expt }, \Gamma}$, are measured or calculated for the corresponding flow condition $\Gamma$. The regularization term, $J_{r e g}$, is not affected by the new design.

The new experimental designs were evaluated by simulated data. In this report, we demonstrate the design that combines flow conditions $\mathrm{A}$ and $\mathrm{B}$ (denoted by $\mathrm{A}+\mathrm{B}$ ). For simplicity, this demonstration was done using the case with two in-plane spatial coordinates, although the same work could be done with the three-dimensional representation. We generated a true permeability distribution (Fig. 33), and the velocity imaging experiment was simulated for the flow conditions A and B. Random measurement error with $1.0 \%$ noise was added to the simulated true velocities to generate simulated data. The simulated velocity data corresponding to the flow conditions A and $\mathrm{B}$ are illustrated in Fig. 34. The permeability distribution was estimated for three kinds of experimental designs. Two of them use either one of the velocity data A and B, and the other one uses the two sets of data together. The estimated permeability distributions are presented in Fig. 35 and their accuracies are evaluated in Table 10.

The accuracy of the permeability distribution was significantly improved with experimental design $\mathrm{A}+\mathrm{B}$ compared to the other designs that use only one experiment. It is notable that the fitting to the data for the design $\mathrm{A}+\mathrm{B}$, represented by the velocity RMSE, is comparable to the other two designs while the accuracy of the estimation is significantly improved.

The contour plots of the permeability distributions (Fig. 35) also show the improvement of the estimation with the design $\mathrm{A}+\mathrm{B}$. The estimate is not exactly the same as the true distribution because we use finite number of velocity data which are superficial averages of discrete volume elements. 

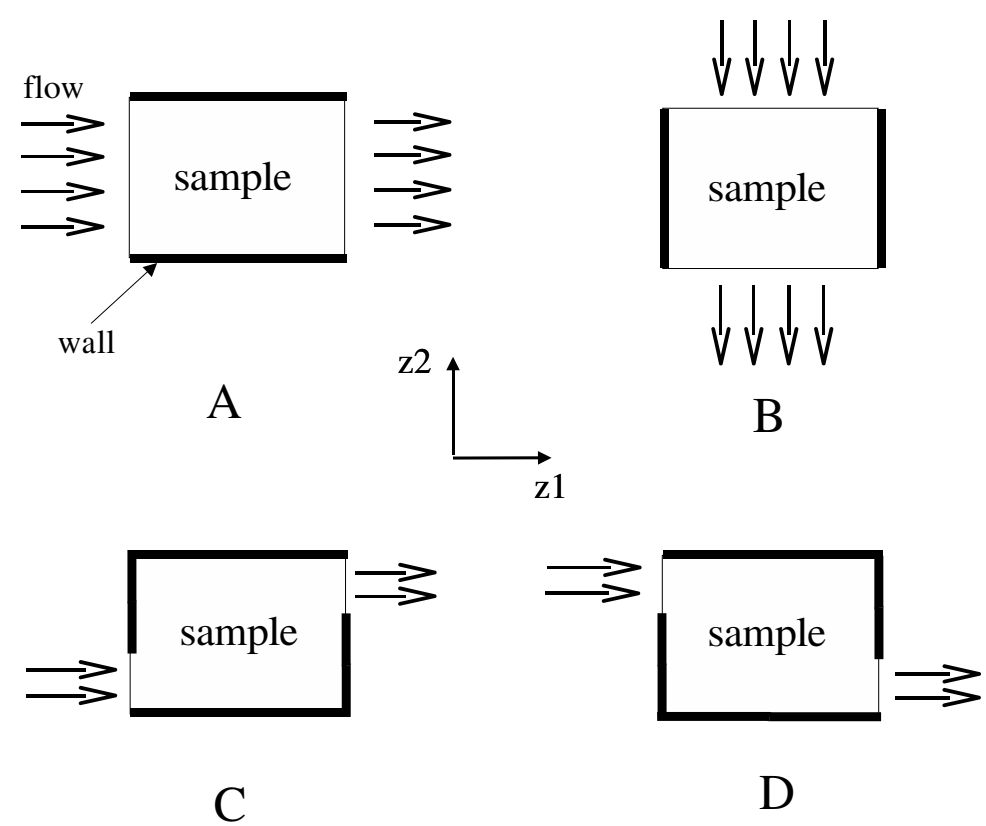

Figure 32: Examples of flow conditions

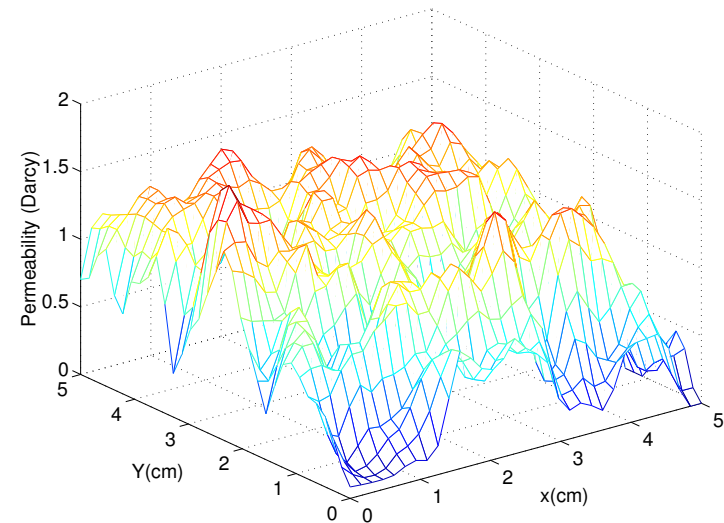

Figure 33: True permeability distribution

\begin{tabular}{||c|c|c||}
\hline Experimental Design & RMSE(v) & RMSE(k) \\
\hline \hline A & 0.0137 & 0.130 \\
\hline B & 0.0116 & 0.147 \\
\hline A + B & 0.0130 & 0.0742 \\
\hline \hline
\end{tabular}

Table 10: RMSE of the calculated velocity and the estimated permeability distribution 
A

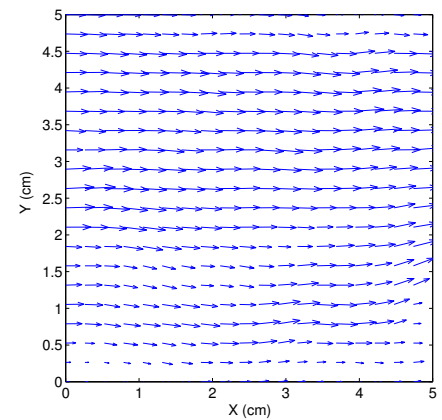

B

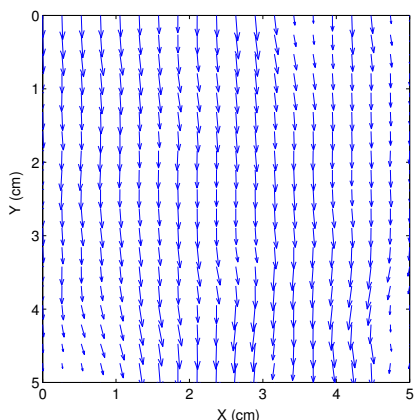

Figure 34: Simulated velocity data

True permeability

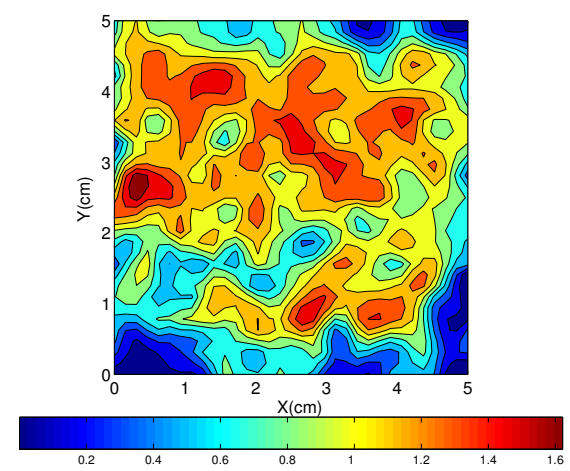

$\mathrm{B}$

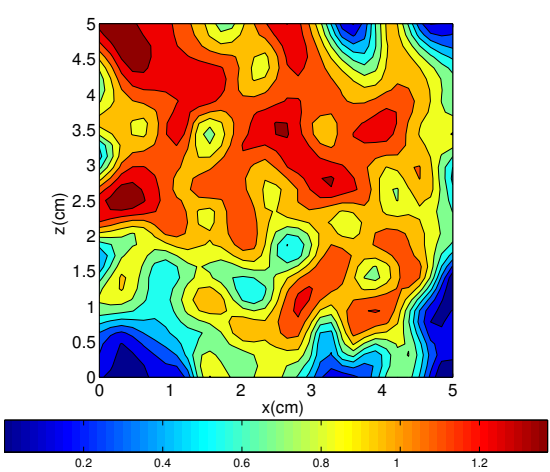

A

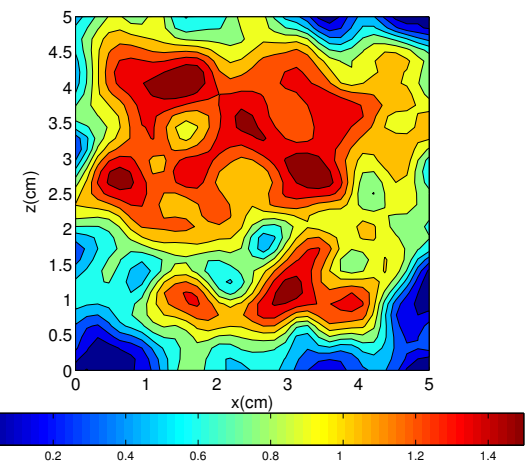

$\mathrm{A}+\mathrm{B}$

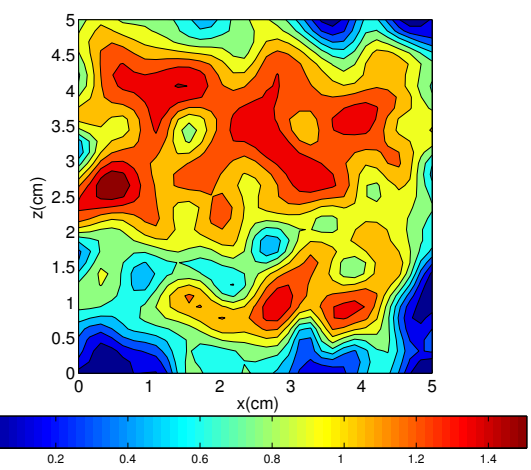

Figure 35: Estimated permeability distribution with different experimental designs 


\subsection{Conclusions}

We have developed a method to determine, for the first time, spatially resolved permeability distributions within samples of permeable media. The method utilizes MRI for the determination of the velocity distribution and the solution of an associated inverse problem to estimate the permeability distribution from the velocity.

The method was successfully demonstrated with experiments conducted on Bentheimer sandstone samples. Unfortunately, we were not able to measure the velocity distributions within the reservoir samples due to the relatively greater rate of relaxation and additional timing requirements of the PFGSTE velocity imaging protocol, as compared to the CPMG imaging sequence used for determining porosity and saturation. Successful applications with these types of samples may still be possible with the identification of a suitable fluid that does not have such a high rate of relaxation when saturating the samples, as does water. The method is likely suitable for many carbonates since saturating water tends to exhibit smaller relaxation rates than with sandstones.

We have proposed new experimental designs that will provide significantly more accurate estimates of permeability distributions for samples or experiments that are amenable to reliable determination of velocity distributions. 


\section{Determination of Multiphase Flow Properties}

Relative permeabilities are determined by conducting experiments involving the flow of two fluid phases, and determining the relative permeabilities from solution of an associated estimation (inverse) problem utilizing a mathematical model of the experiment. This problem has been investigated extensively by the PI and collaborators, who first posed the estimation problem using nonparametric principles (Kerig and Watson 1986; Watson et al. 1988). They have developed a general approach to formulate and solve the associated estimation problem for determining multiphase flow properties utilizaing any experimental design, and to evaluate the experimental design (Watson et al. 1998). To date, applications of this work have been based on one-dimensional models of the displacement experiments. In this work, we develop the means to include instrinsic porosity and permeability distributions for estimating multiphase flow properties from displacement experiments and advance the use of complete, three-dimensional mathematical modeling of displacement experiments.

\subsection{Introduction}

Significant advances in determining multiphase flow properties were reported by Kulkarni etal. (1998). Their analysis includes the effects of capillary pressure, which is necessary when experiments are conducted under the capillary-dominated flow conditions that are representative of reservoir conditions. They estimated capillary pressure together with relative permeability functions, and developed measures of the accuracy with which the functions are determined.

Kulkarni et al. (1998) introduced a new experimental design that greatly improves the accuracy with which the multiphase flow properties can be estimated. Rather than using a single injection

rate, as is done conventionally, they used several successive rates. This resulted in more accurate estimates over a greater range of saturation. In addition to the conventional measurements of pressure drop and production, which are acquired outside of the sample, they included measurements of saturation distributions obtained using MRI (see also Mejia et al. 1995, who used X-ray CT scanning). Spatially resolved measurements can provide much more information than the conventional measurements that are only sensitive to the integrated (or average) states of the entire sample.

Kulkarni et al. (1998) evaluated their estimates by comparing the values calculated using the model of the experiment to the corresponding measured quantities. The conventional measurements of pressure and production were predicted very precisely. However, the measured and calculated 
saturation distributions were not as consistent. It was hypothesized that the discrepancy was due to the failure to account for possible spatial variations in the porosity and the permeability, which were represented as a one-dimensional function (along the axial direction) and as the uniform apparent value, respectively, in the mathematical model of the experiment.

The intrinsic properties, when known, can be incorporated into the estimation problem, as discussed in the following section. It is expected that subsequent estimates of the multiphase flow properties will be more accurate than if the porosity and permeability were only represented with average or apparent values. We explore this aspect in Section 7.3. Then, we present experimental work directed to demonstrating the incorporation of intrinsic permeability and porosity into the estimation process.

\subsection{Methodology}

The multiphase flow properties are estimated by determining the relative permeability and capillary pressure functions that minimize a performance index based on differences between the measured and calculated quantities. The calculated quantities are determined from solution of the model of the displacement experiment with specified properties.

The performance index is:

$$
J=\left[\mathbf{Y}^{\text {data }}-\mathbf{Y}^{\text {calc }}\right]^{T} \mathbf{W}\left[\mathbf{Y}^{\text {data }}-\mathbf{Y}^{\text {calc }}\right] .
$$

The data will typically include the pressure drop and production and, for our work, values of saturation that represent averages corresponding to successive regions in the axial direction (Kulkarni et al. 1998).

The model for the experiment follows from the state equations introduced earlier (see Section 2.1). Using the generalized Darcy expression (Eq. 4) to eliminate the velocity in Eq. 3, we obtain the following equation for each fluid phase:

$$
\frac{\partial}{\partial t}\left(\phi \rho_{i} s_{i}\right)=\nabla \cdot\left[\frac{k k_{r i} \rho_{i}}{\mu_{i}}\left(\nabla p_{i}-\rho_{i} \mathbf{g}\right)\right], \quad i=1,2 .
$$

The boundary conditions and initial conditions are specified on the basis of the particular experiment used. We consider here a drainage experiment in which the nonwetting fluid is injected into a sample initially saturated with the wetting fluid. The outlet is maintained at a given pressure. These boundary conditions are specified by:

$$
\int_{S_{\text {ent }}} \frac{k k_{r 1} \rho_{1}}{\mu_{1}} \mathbf{n}_{1} \cdot\left(\nabla p_{1}-\rho_{1} \mathbf{g}\right) d \mathbf{z}=q_{1, \text { ent }}
$$




$$
\begin{gathered}
\left.\mathbf{n}_{1} \cdot\left(\nabla p_{2}-\rho_{2} \mathbf{g}\right)\right|_{S_{\text {ent }}}=0 \\
\left.p_{1}\right|_{S_{\text {exit }}}=\left.p_{2}\right|_{S_{\text {exit }}}=p_{\text {out }}
\end{gathered}
$$

Here, $S_{\text {ent }}$ and $S_{\text {exit }}$ represent inlet and outlet faces, repectively, $\mathbf{n}_{1}$ is a unit vector normal to those surfaces, $q_{1, \text { ent }}$ is the injection flux of phase 1 at $S_{\text {ent }}$, and $p_{\text {out }}$ is the ambient pressure. The boundary conditions implement the capillary end-effect discussed in Dullien (1992). The initial conditions are

$$
\begin{gathered}
s_{2}(\mathbf{z})=1 \\
p_{2}(\mathbf{z})=p_{\text {out }}
\end{gathered}
$$

The fluid properties are determined independently. Once all the permeable media properties are specified (i.e., porosity, permeability, two relative permeabilities, and capillary pressure), Eqs. 91, 5 , and 6, with pertinent boundary and initial conditions, can be solved for the pressures and saturations of each of the fluid phases, as a function of position and time.

In keeping with our nonparametric approach to these functional estimation problems, we use B-splines to represent the the relative permeability and capillary pressure functions:

$$
\begin{aligned}
k_{r i}\left(s_{i}\right) & =\sum_{j=1}^{N_{i}} c_{j}^{i} B_{j}^{m}\left(s_{i}, \mathbf{x}^{i}\right), \quad i=1,2 \\
p_{c}\left(s_{1}\right) & =\sum_{j=1}^{N_{c}} c_{j}^{c} B_{j}^{m}\left(s_{1}, \mathbf{x}^{c}\right) .
\end{aligned}
$$

We incorporate inequality constraints into the minimization problem specified by the performance index (Eq. 90):

$$
\mathrm{Gc} \geq \mathbf{g}_{0}
$$

The inequality constraints are chosen to insure that physically realistic estimates are obtained at each step of the minimization procedure. We use a trust-region based, linear-inequality constrained Levenberg-Marquardt algorithm (Richmond 1988) to solve this minimization problem. In order to choose a suitable partition-numbers and location of knots-for the multiphase functions, we solve a sequence of minimization problems with increasing values of the spline dimensions. We generally look for the first solution for which satisfactory residuals-differences between measured and calculated values-are obtained. By starting with very few degrees of freedom, and beginning each successive problem with the prior estimates, our method is robust and efficient.

This formulation is quite general in that it can be used with any displacement experiment, and with any estimates for the porosity and permeability. At issue is the accuracy of the model for 
representing the experiment. In particular, it is desirable that the specified boundary and initial conditions are consistent with the experiments, and that the porosity and permeability are accurate. If intrinsic porosity and permeability are known, they should be included in the estimation process. If average or apparent values are used, it is expected that there will be errors in the estimates due to the inaccuracies in the model of the experiment.

In previous work for determining multiphase flow properties, one-dimensional models of the experiment have been employed. Conventionally, it has been assumed that the properties are homogeneous, and thus represented as single values. One-dimensional variations in properties in the axial direction can be included in the estimation process with one-dimensional models, as it has been for experiments with composite core samples, each having uniform values, (Mejia et al. 1995) and for porosity as a function of axial position, as determined with MRI or CT scanning (Kulkarni etal. 1998; Mejia etal. 1995). Note also that if there are gravity effects due to a difference in the densities of the fluids, a one-dimensional model is appropriate if the experiment is conducted vertically.

We do know that porosity and permeability vary spatially, and when known, those properties can be taken into account in the model of the experiment. However, a fully three-dimensional mathematical model of the experiment is required. In our prior recent work, we have used a commercial software package, Sendra (Petec Software \& Services 2000), which allows for simulation with up to two spatial dimensions. In this work, we extended the capabilities so that three-dimensional displacement experiments can be modeled. The details and validation of that implementation are provided elsewhere (Uh et al. 2002; Watson et al. 2002; Xue 2004).

\subsection{Value of including intrinsic permeability}

We have investigated potential errors which may be encountered in estimating multiphase flow properties when simplified representations of the intrinsic properties are used. We illustrate the method here. Further details are available elsewhere (Watson et al. 2002; Valestrand 2002).

We simulated displacement experiments by solving the models equations (see Eqs. 91-93, 5, and 6) with specified properties. The multiphase flow properties (taken from Kulkarni et al. (1998)) and

the (absolute) permeability distribution used are shown in Figs. 36-38. A uniform value of porosity was used. Simulated experimental data were created by adding random errors to the calculated pressure drop and saturation values. The errors were obtained with a random number generator using a normal distribution with the following variances: $\sigma_{\Delta p}^{2}=1.0 \mathrm{~Pa}^{2}$ and $\sigma_{s_{2}}^{2}=2.3 \times 10^{-4}$. 
We then performed two estimations using the simulated experimental data. In Case 1, we used the specified (true) porosity and permeability properties. In Case 2, we represented the permeability using the harmonic average value of the specified distribution (see Fig. 38). This represents the value that would be measured from a conventional experiment to determine the (apparent) permeability.

The simulated data and corresponding calculated pressure drop values obtained using the estimated relative permeabilities are shown in Figs. 40 and 41 for Cases 1 and 2, respectively. The use of the true permeability distribution provides the most precise fit to the data, although the match associated with Case 2 may be taken to be acceptable. The simulated data and corresponding calculated saturation distributions are shown in Figs. 42 and 43 for Cases 1 and 2, respectively. While a suitable match of the saturation distribution is obtained using the correct permeability, that obtained using the corresponding harmonic-averaged value is not satisfactory. Evidently, there are no physically realistic relative permeability curves that allow reconciliation of the data. Note that smallest value of water saturation attained during the simulated experiment is 0.53 (see saturation data in Fig. 42). Thus the experiment provides no information about the relative permeability functions corresponding to smaller values of saturation.

The estimates for the relative permeabilities obtained for the two cases are shown in Fig. 39. This shows that, for Case 1, the multiphase flow properties are accurately estimated over the saturation region represented by the experiment; however, there are significant errors in the estimates for Case 2.

This example illustrates that errors in the estimated multiphase flow properties may be encountered when insufficient resolution is used for the absolute permeability. It also illustrates the importance of validation of the estimates, as put forth by Kulkarni etal. (1998). The estimates for Case 1 are validated, while those for Case 2 are not; consequently, the estimates for Case 1 cannot be rejected on the basis of the precision of the match, while those for Case 2 should be rejected. Finally, it illustrates the value of measured saturation distributions for use in estimating multiphase flow properties. 


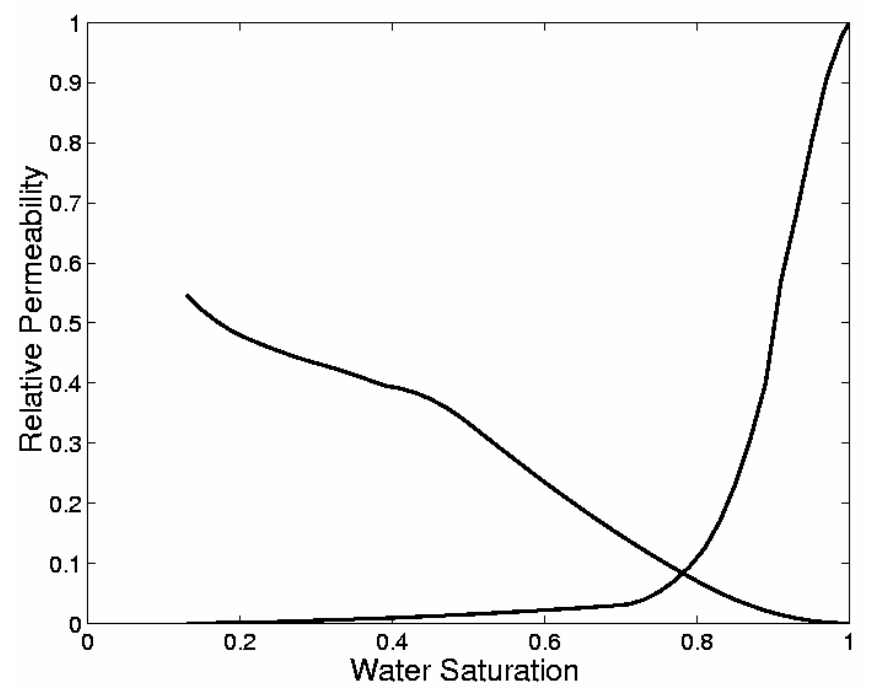

Figure 36: True relative permeability

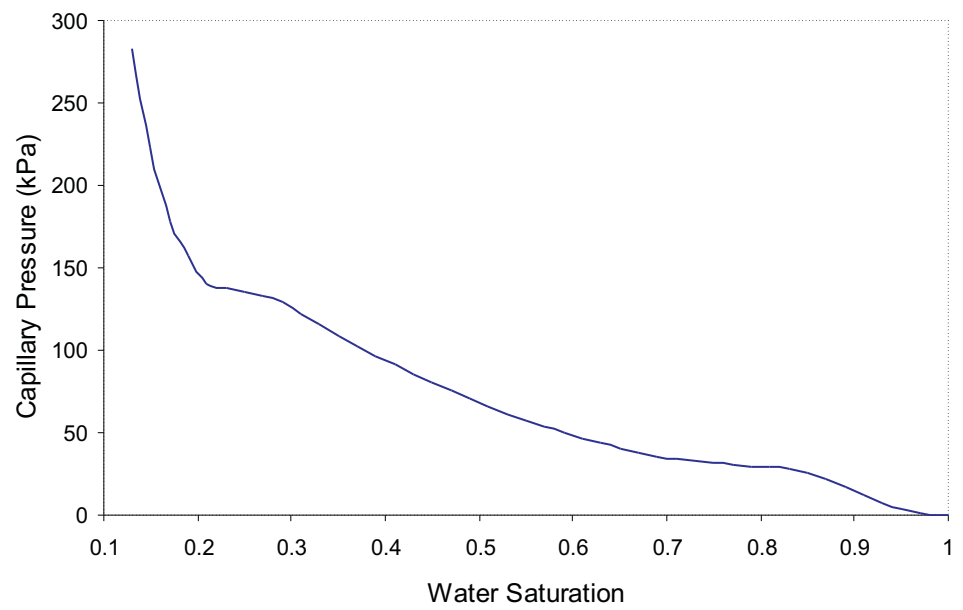

Figure 37: Capillary pressure curves used in the estimation process 


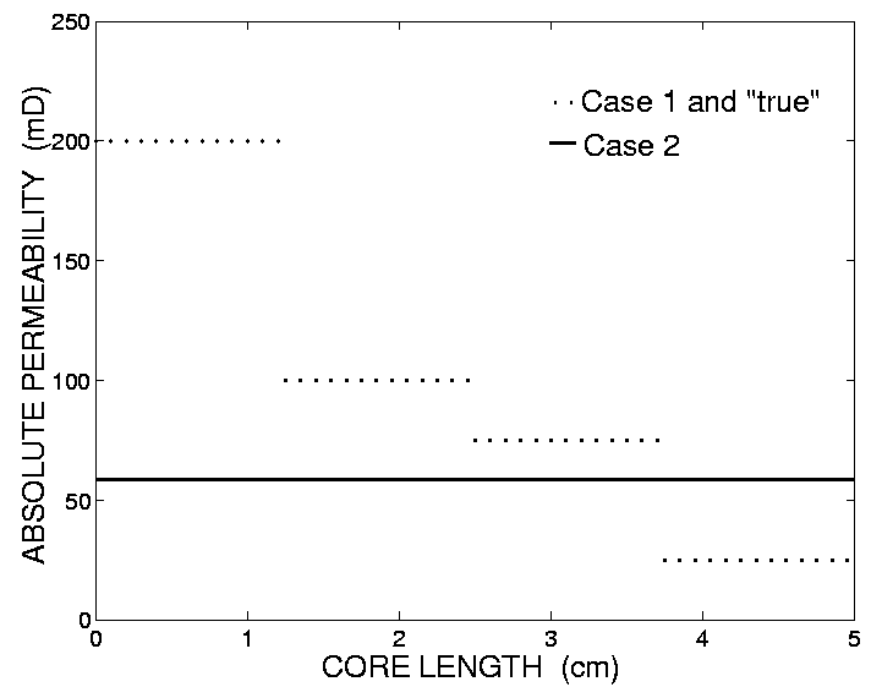

Figure 38: Absolute permeability distributions used in the estimation

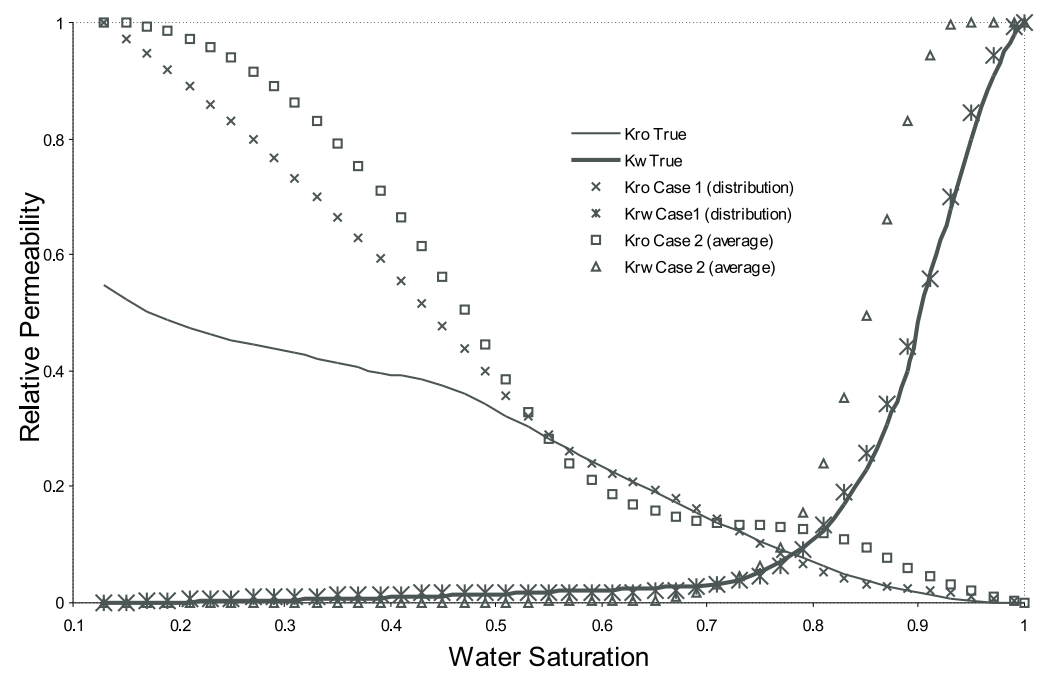

Figure 39: Estimated relative permeability functions for two cases 


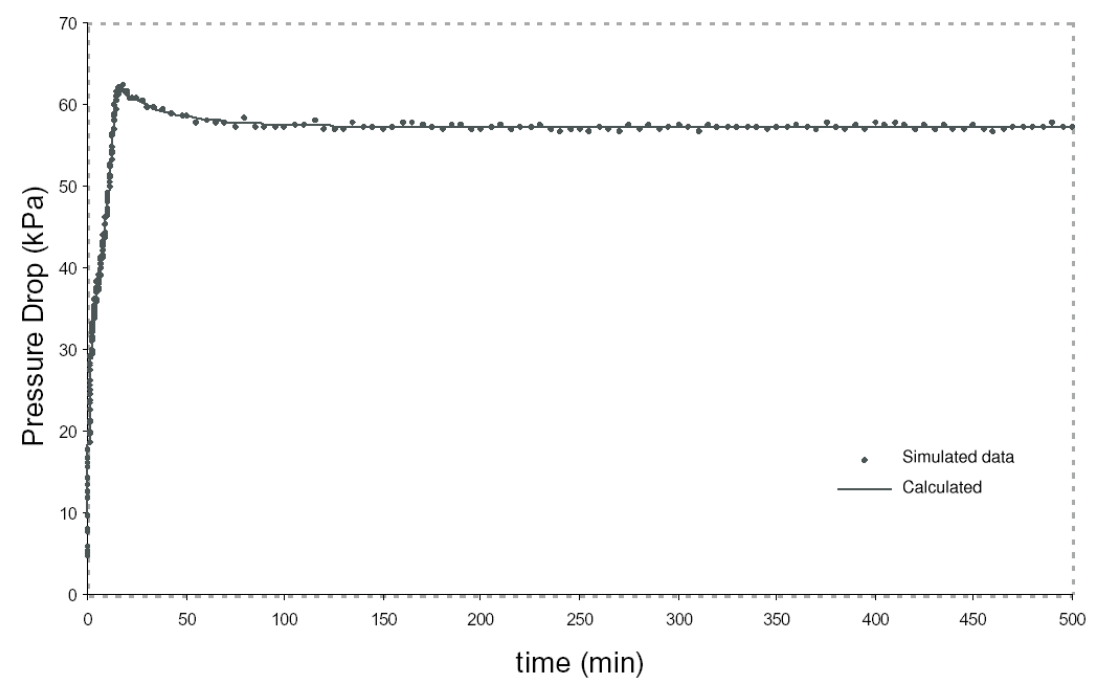

Figure 40: Simulated pressure drop data and the calculated values for Case 1

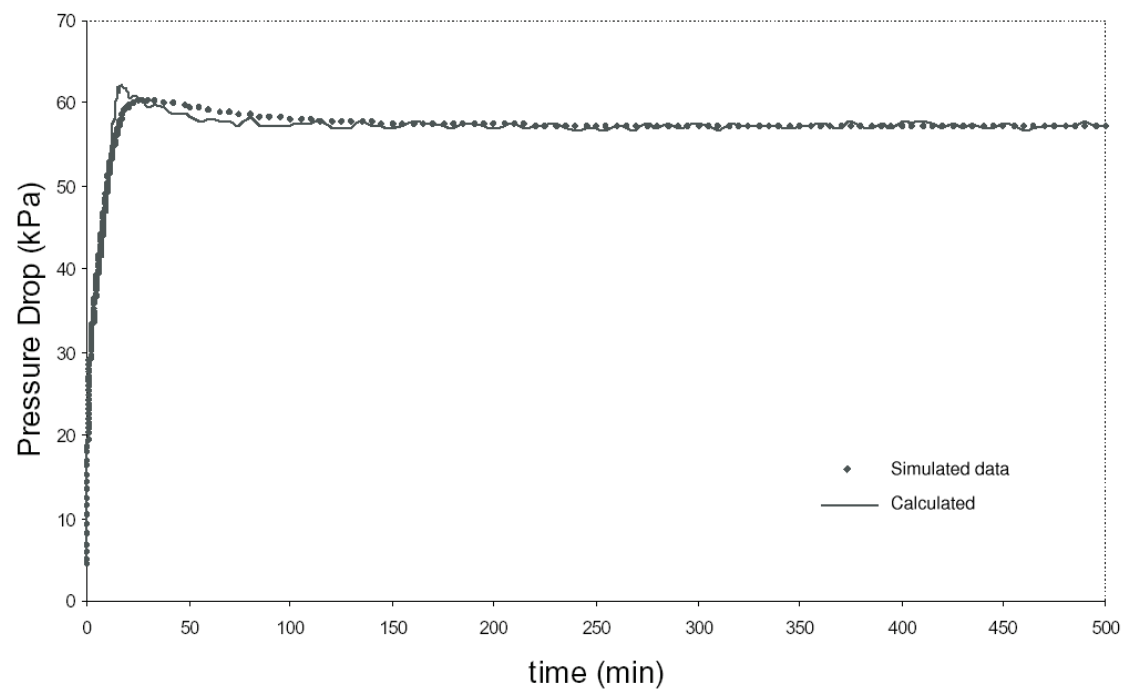

Figure 41: Simulated pressure drop data and the calculated values for Case 2 


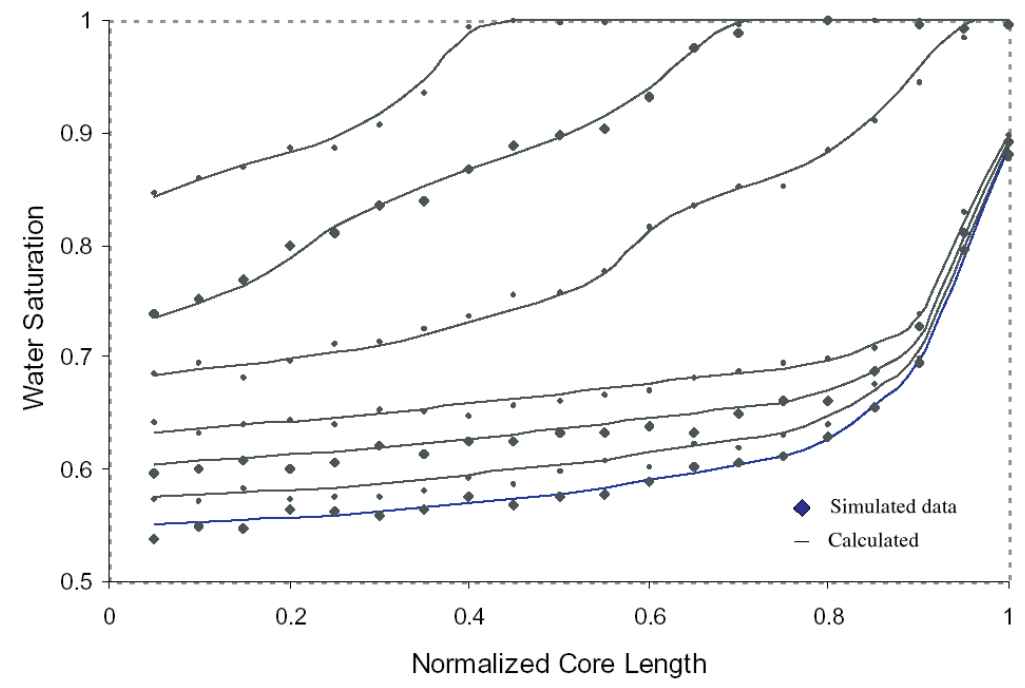

Figure 42: Simulated saturation data and the calculated values for Case 1

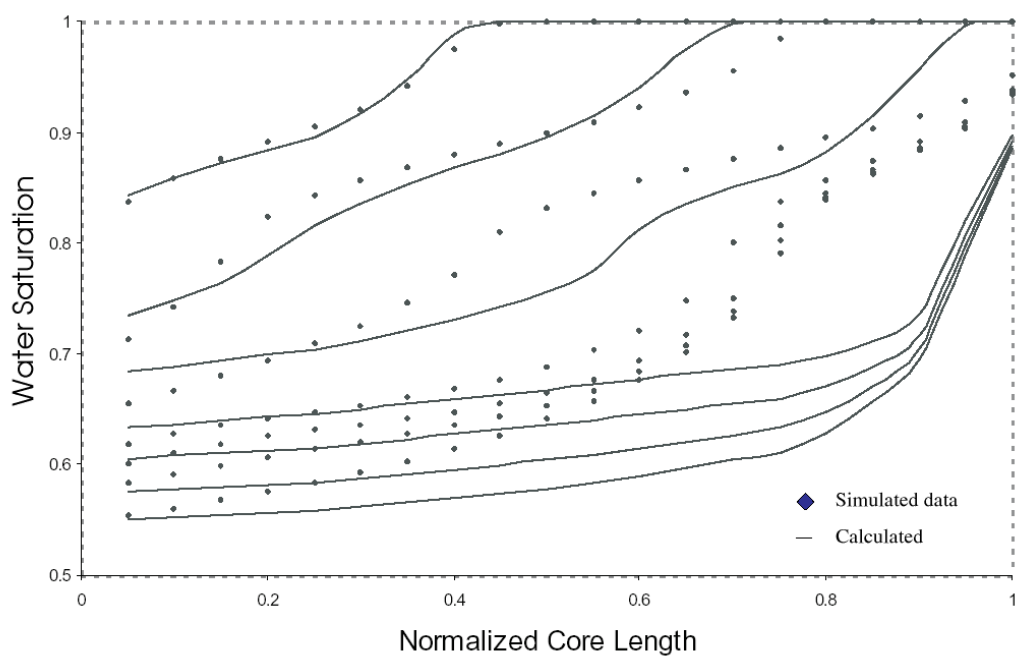

Figure 43: Simulated saturation data and the calculated values for Case 2 


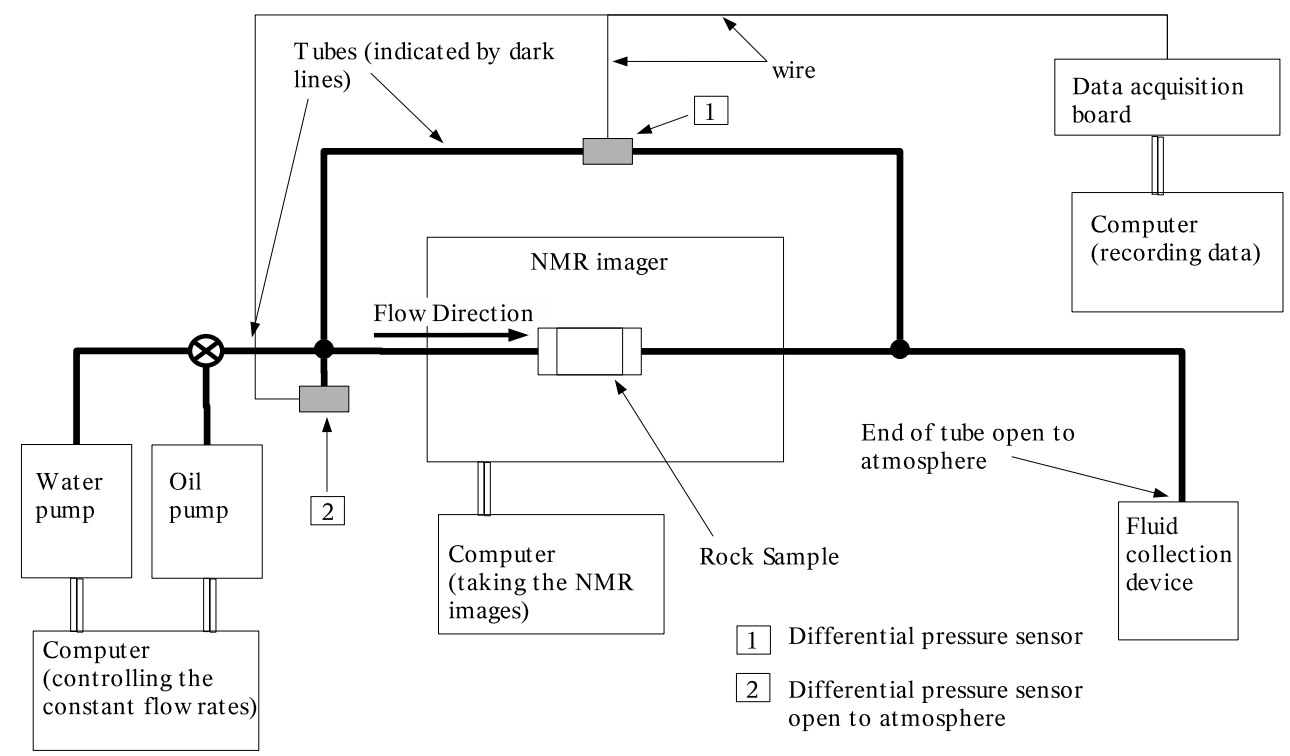

Figure 44: Schematic of experimental apparatus for displacement experiments

\subsection{Displacement experiments}

A number of displacement experiments were run with the objective of demonstrating the use of intrinsic properties and three-dimensional modeling of the experiment for the determination of relative permeability and capillary pressure functions. We summarize these efforts in this section.

\subsubsection{Experimental setup}

A schematic of our experimental apparatus for conducting displacement experiments is shown in Fig. 44. A constant, continuous flow is delivered by dual-piston Quizix@ QL-700 pumps, which are situated outside of the imaging enclosure (details on the configuration of the imager are provided in Watson etal. (2002). Honeywell ${ }^{\circledR}$ differential pressure transducers are used to measure the pressure drop across the sample, and the pressure upstream of the sample, relative to atmospheric pressure, which is measured independently. In some experiments, we used a production device to monitor the production of the displaced phase (Phan et al. 2001).

The sample holder device is shown in Fig. 45. It is necessary that the holder contains no ferrous (magnetic) materials. The picture shows that the sample is potted with epoxy within a plexiglass tube. Nylon bolts are used to attach the end caps to the sample. A water-tight seal is maintained with $0.79 \mathrm{~mm}$ rubber gaskets. One of the endcaps has a small enclosure in which a reference sample 


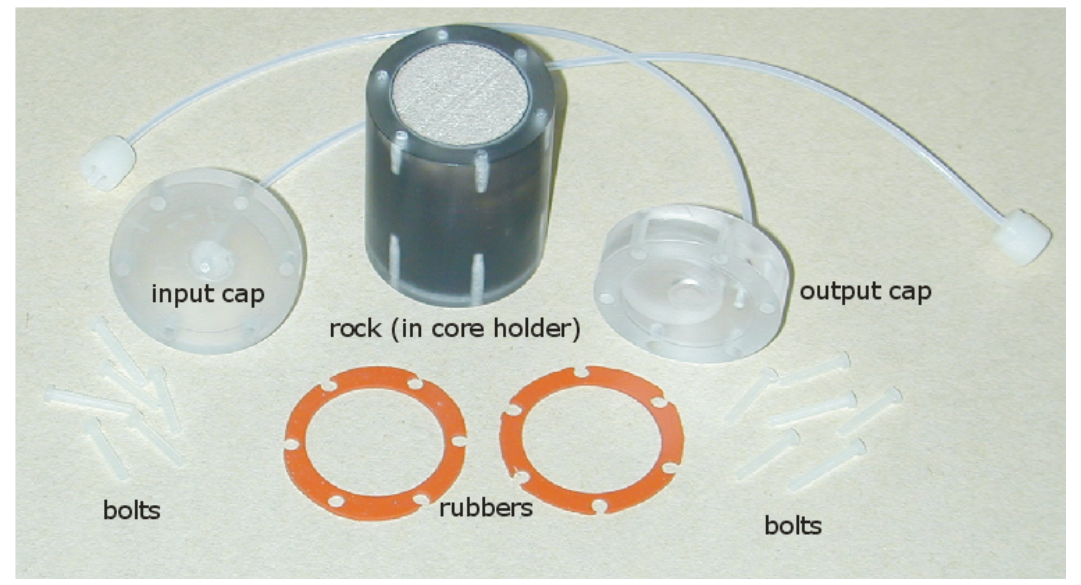

Figure 45: Sample holder device

can be included. Nylon tubing and fittings are used.

It is desirable that the sample resides completely within the high-linearity region of the radiofrequency $(\mathrm{RF})$ coil as well as the gradient coils. For our current equipment, this corresponds to a cylindrical region $3 \mathrm{~cm}$ in length and $3.5 \mathrm{~cm}$ in diameter. Since we wanted measurements of permeability for our sample, the length was further limited by the coil we used for that experiment, which is shorter. We ultimately used samples that were $2.5 \mathrm{~cm}$ in diameter and $2 \mathrm{~cm}$ in length. We used the sample positioner described earlier (see Fig. 19) so that we can align the sample identically for the porosity, permeability, and displacement experiments, which were typically conducted at different times. A schematic of the sample as it resides within the RF-coil inside the bore of the MRI is shown in Fig. 46.

\subsubsection{Experimental design}

We conducted three different displacement experiments using two different Bentheimer samples. For each of the experiments, we conducted either two or three sequential cycles marked by injection of different fluids. In the first experiment (Experiment I), we conducted a drainage cycle, followed by an imbibition cycle and a second drainage cycle. The objective was to obtain estimates of the multiphase flow properties for primary and secondary drainages, and primary imbibition. In the second and third experiments (Experiment II and III), only primary drainage and imbibition cycles were conducted. For each of the drainage cycles, several successively increasing injection rates were used, while only a single rate was used for the imbibition cycles. Details associated with these experiments are provided in Table 11. 


\begin{tabular}{|c|c|c|c|}
\hline Experiment & 1st cycle & 2nd cycle & 3rd cycle \\
\hline \multirow{5}{*}{ I } & Drainage & Imbibition & Drainage \\
\hline & $0.5 \mathrm{ml} / \min (0 \mathrm{~min})$ & $0.5 \mathrm{ml} / \mathrm{min}(750 \mathrm{~min})$ & $0.5 \mathrm{ml} / \mathrm{min}(1375 \mathrm{~min})$ \\
\hline & $1.0 \mathrm{ml} / \mathrm{min}(196 \mathrm{~min})$ & $1.0 \mathrm{ml} / \mathrm{min}(901 \mathrm{~min})$ & $1.0 \mathrm{ml} / \mathrm{min}(1537 \mathrm{~min})$ \\
\hline & $2.0 \mathrm{ml} / \mathrm{min}(392 \mathrm{~min})$ & $2.0 \mathrm{ml} / \mathrm{min}(1055 \mathrm{~min})$ & $2.0 \mathrm{ml} / \mathrm{min}(1686 \mathrm{~min})$ \\
\hline & $3.0 \mathrm{ml} / \mathrm{min}(570 \mathrm{~min})$ & $3.0 \mathrm{ml} / \mathrm{min}(1204 \mathrm{~min})$ & $3.0 \mathrm{ml} / \mathrm{min}(1832 \mathrm{~min})$ \\
\hline \multirow{5}{*}{ II } & Drainage & Imbibition & \\
\hline & $0.3 \mathrm{ml} / \mathrm{min}(0 \mathrm{~min})$ & $0.6 \mathrm{ml} / \mathrm{min}(575 \mathrm{~min})$ & \\
\hline & $0.6 \mathrm{ml} / \mathrm{min}(139 \mathrm{~min})$ & & \\
\hline & $0.9 \mathrm{ml} / \mathrm{min}(214 \mathrm{~min})$ & & \\
\hline & $1.2 \mathrm{ml} / \mathrm{min}(290 \mathrm{~min})$ & & \\
\hline \multirow{6}{*}{ III } & Drainage & Imbibition & \\
\hline & $0.3 \mathrm{ml} / \mathrm{min}(0 \mathrm{~min})$ & $0.3 \mathrm{ml} / \min (1144 \mathrm{~min})$ & \\
\hline & $0.5 \mathrm{ml} / \mathrm{min}(130 \mathrm{~min})$ & $0.5 \mathrm{ml} / \mathrm{min}(1376 \mathrm{~min})$ & \\
\hline & $0.75 \mathrm{ml} / \mathrm{min}(224 \mathrm{~min})$ & & \\
\hline & $1.0 \mathrm{ml} / \mathrm{min}(294 \mathrm{~min})$ & & \\
\hline & $1.25 \mathrm{ml} / \mathrm{min}(352 \mathrm{~min})$ & & \\
\hline
\end{tabular}

Table 11: Design of displacement experiments (The time in parenthesis indicates when the corresponding flow rate was started based from the beginning of the experiment) 


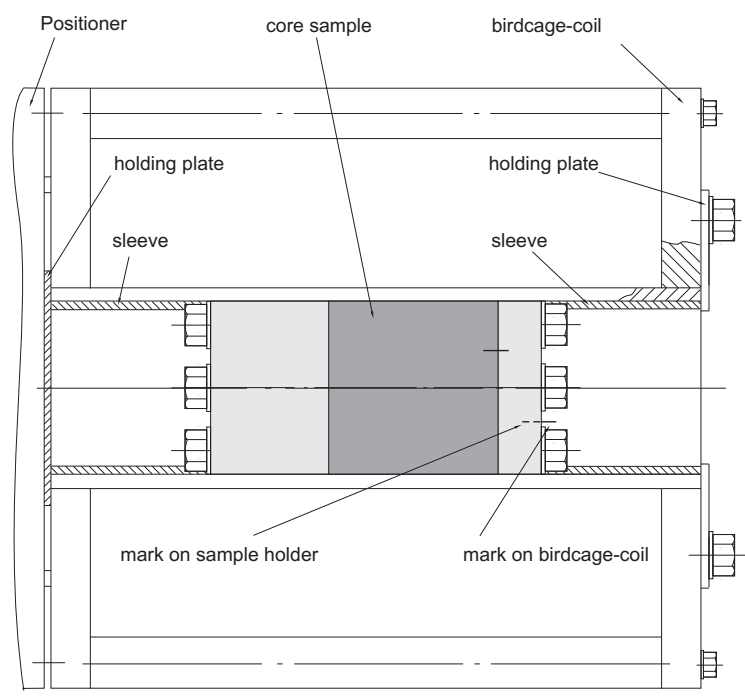

Figure 46: Schematic of the sample as it resides within the RF-coil inside the MRI bore

\begin{tabular}{||c|c|c||}
\hline \hline Name & Specific gravity & Viscosity $(\mathrm{cP})$ \\
\hline \hline Deuterium oxide $\left(\mathrm{D}_{2} \mathrm{O}\right)$ & 1.11 & 1.26 \\
\hline Soltrol $^{\circledR} 130$ & 0.763 & 2.08 \\
\hline \hline
\end{tabular}

Table 12: Basic properties of fluids (at $18^{\circ} \mathrm{C}$ )

We used deuterium oxide $\left(\mathrm{D}_{2} \mathrm{O}\right)$ for the wetting fluid phase, and an isoparaffin oil (Soltrol $\left.{ }^{\circledR}\right) 130$, Phillips Petroleum Co.) for the nonwetting fluid phase. The basic properties of those fluids are presented in Table 12. With NMR, we observe the protons in the isoparaffin oil. For Experiments I and III, the samples were initially fully saturated with $\mathrm{D}_{2} \mathrm{O}$. At the conclusion of the first experiment, we cleaned the sample with solvents such as ethyl alcohol, but the sample evidently retained some oil which was observed at the beginning of Experiment II.

We used MRI to monitor one-dimensional saturation profiles throughout the experiments. Each profile can be acquired in less than a second. Each flow rate was maintained up to steady state conditions, as identified by observing no further changes in the saturation distributions. At that point, three dimensional saturation distributions were measured. Each scan required about five minutes. 


\subsubsection{Results and discussion}

Each of the experiments was extensively analyzed using the method described previously in Section 7.2. The estimation problem is very non-linear, so it is best to start with a problem that can be solved relatively quickly in order to identify good initial guesses for the full, three-dimensional estimation problems. Thus, we used one-dimensional simulations, initially with uniform values of (apparent) permeability and (average) porosity, and then with one-dimensional representations of the properties as a function of the axial position. We also used combinations of pressure drop and production (obtained by mass balances with an associated integral of the saturation), as for conventional experiments, or pressure drop with saturation distributions, as well as pressure drop, saturation, or production alone. While we could generally reconcile any single type of data, we were never able to reconcile pressure with production or saturation. The lack of fit seemed too great to be the result of approximating permeability and porosity distributions with one-dimensional profiles formed by averaging longitudinally. We carefully evaluated all our experimental protocols, and ran the several different experiments in the hopes of finding any experimental problems.

We take great care to avoid the introduction of any air bubbles into the system. To do this, we mount the sample within the core holder, vacuum saturate it, and close valves that are attached to short tubes at either end of the sample. We then make sure that the line we attach from the pump contains no air bubbles.

In each of the experiments, we observed some pressure anomalies in the initial injection period. An example is provided in Fig. 47. Overall, the trace shows the features we expect: the pressure drops increase sharply with each increase in the injection rate, and then fall with time. The relatively large spikes occur when the pump shifts to the alternate piston. From the beginning, up until 70 minutes, we see some fairly large, unexpected excursions in pressure.

We believe now that this is due to redistribution of fluids within the inlet and outlet end caps. Based on the Young-Laplace equation, some of these fluctuations may be of the size associated with the capillary pressure for a water bubble within the small, $0.8 \mathrm{~mm}$ diameter tubing that we use to minimize dead times in our experiment.

To investigate this further, we observed the first echoes associated with the one-dimensional images (see Fig. 48) for Experiment III. In the inlet, outlet, and reference standard, the first echoes are proportional to the amount of fluid since the relaxation time associated with the bulk fluid is considerably longer than the echo time. While we had expected that the wetting fluid would be 


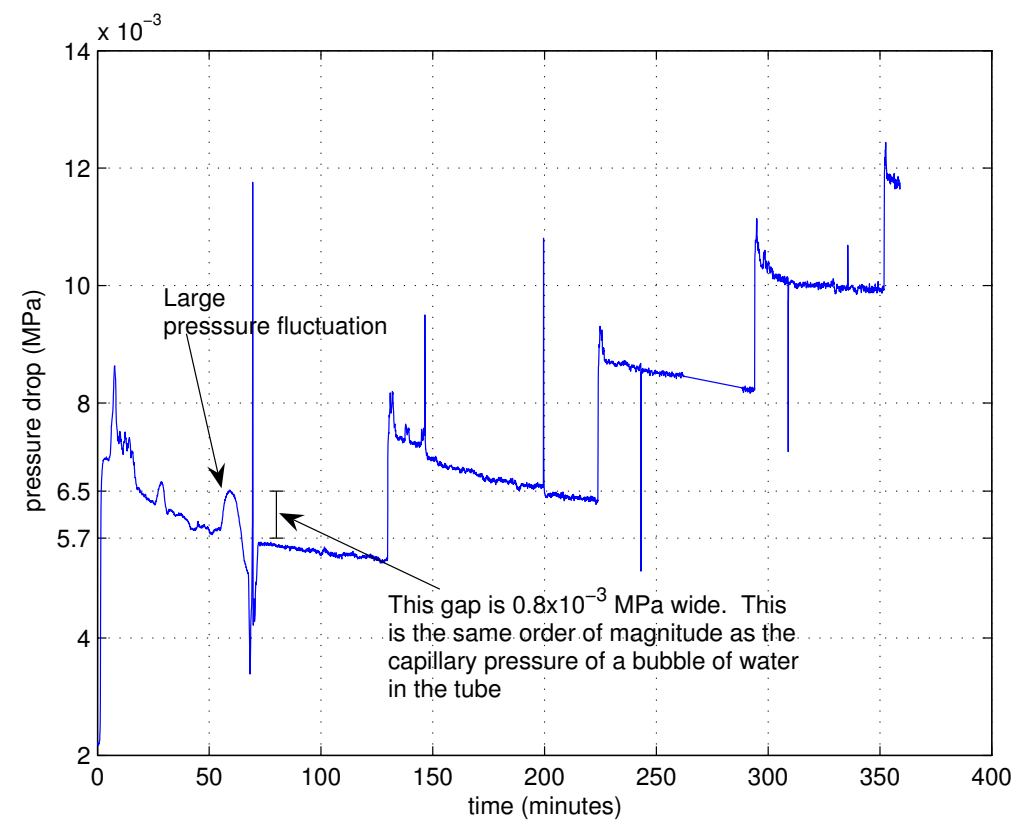

Figure 47: Pressure drop data of the primary drainage cycle of Experiment III

displaced from the inlet gap early in the experiment, so that face would be exposed only to the injection phase, we see that is not the case.

In Experiment II, we filled the inlet gap with small glass beads of diameter $0.1 \mathrm{~mm}$. This did decrease the associated hold-up, but resulted in additional problems with sample preparation together with the need to determine the hydraulic resistance in that region. Most of all, we still observed significant changes in the fluid content of the endcaps with flow rate. This information is provided in Figure 49, which plots the single intensity in the inlet and outlet encaps for different flow rates, and as a function of time at each flow rate. The figure shows that the amount of the nonwetting fluid in the caps changes with time, and with flow rate. Particularly troubling is the inlet cap. It shows that both fluids (oil and water) are within the endcap, which is clearly not consistent with the inlet boundary condition.

The images provided evidence that the boundary conditions associated with the experiment are not matched by the mathematical model. It seems likely, however, that this same problem is being encountered by others in one form or another, but not recognized since conditions within the caps and distributions of saturations within the sample are generally not observed. We used a different endplate design in the experiments reported by Kulkarni et al. (1998). The endplate was flush 


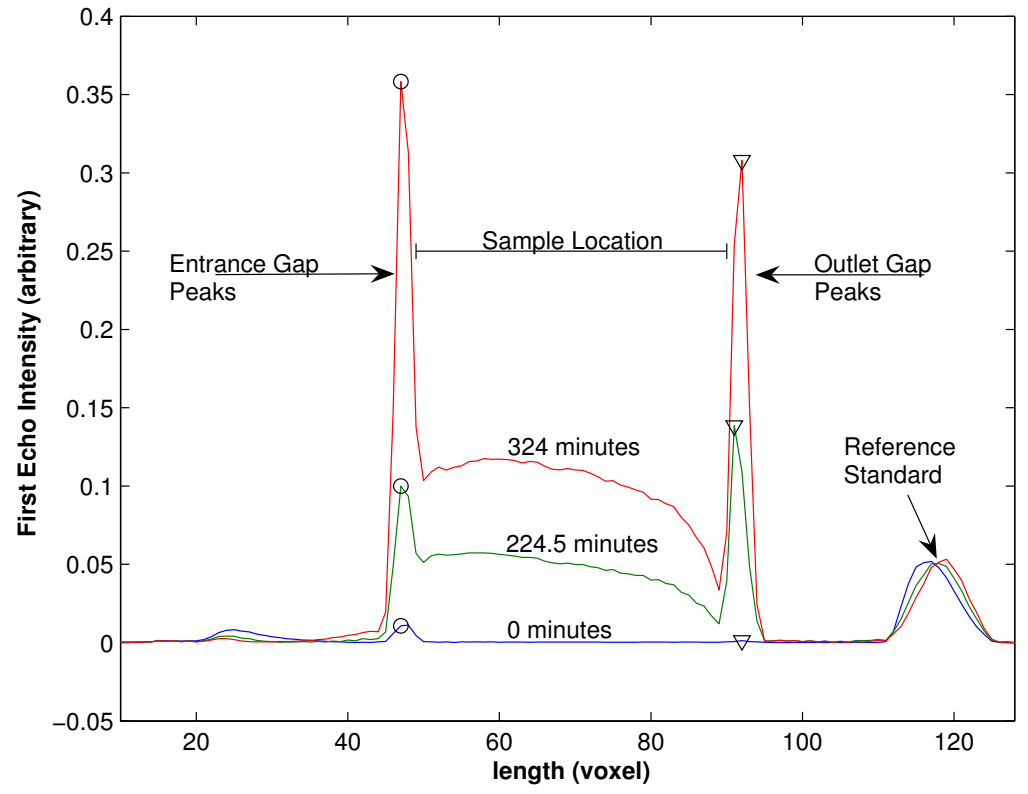

Figure 48: One-dimensional first echo image for Experiment III

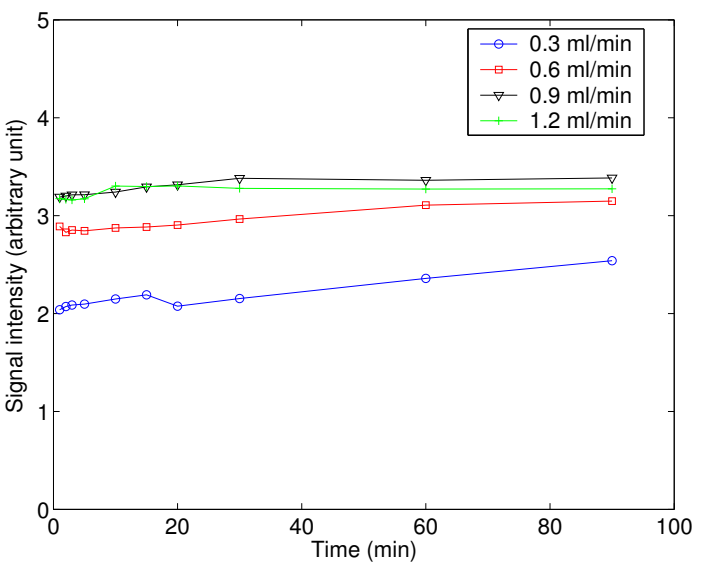

(a) $S_{\text {ent }}$

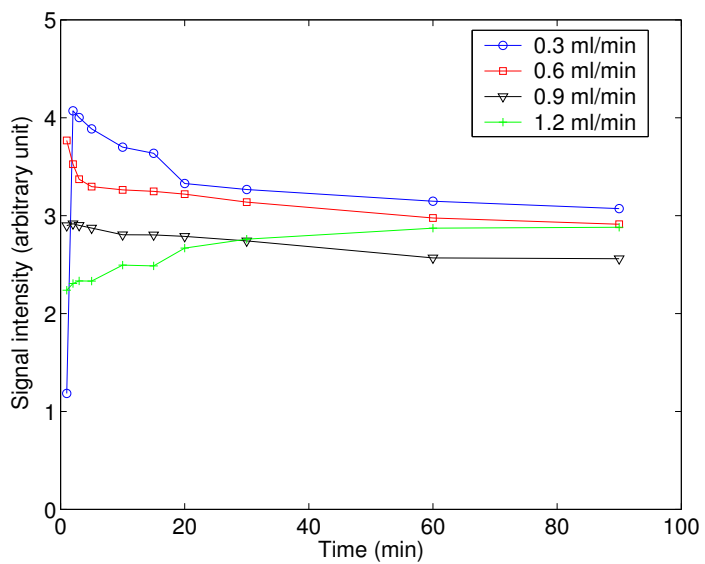

(b) $S_{\text {exit }}$

Figure 49: The signal intensity of bulk fluid within the inlet and outlet endcaps for Experiment II 
against the sample, but contained two circular channels that carried the injection fluid. While the hold up was smaller, it is not clear that this experiment actually matches the boundary conditions either, and the configuration could give rise to saturation distribution differences orthogonal to the axial direction, which would not be detected by one-dimensional images along the axial direction. We also note that the simulated values at the outlet of the sample, which must converge to a single value, according to the usual outlet conditions, don't precisely match the data (see figure 16 in Kulkarni et al. (1998)).

Our three-dimensional saturation imaging results show that saturations within the plane orthogonal to the axial direction are not uniform. This may be the result of property heterogeneity, or in part the result of some complex multidimensional flow which could result from the participation of the fluids in the endcap in the displacement process, as recognized previously (see Figs. 48 and 49). We show the one-dimensional saturation distributions for the primary drainage cycle of Experiment III for various times, corresponding to a different flow rate in each of the Figs. 50-53. (The times presented in the figures are based from the starting of each corresponding flow rate.) The successive increases in the one-dimensional profiles are consistent with expectations. Note also how greater ranges of saturation as accessed with the increasing injection rates.

We also show data obtained from the multi-dimensional imaging experiments conducted after achieving each steady state. We show the saturation values for voxels corresponding to various axial positions, and compute the mean and $90 \%$ residual regions. The saturations indicate a relatively large spread at the higher flow rates, suggesting that the multidimensional simulation we developed may be necessary to suitably model the experiment. We emphasize that, with full three-dimensional modeling of the experiment and the inclusion of intrinsic porosity and permeability, the use of onedimensional saturation profiles obtained with MRI does not imply any simplification of the problem since our measurements represent integrals across the transverse plane corresponding to successive regions in the axial direction. It is important that that corresponding calculated values obtained within the performance index (see Eq. 90) are in fact the same integral representations of the calculated three-dimensional saturation distributions. Note that the multi-dimensional saturations can, and should, be included as data as well. 


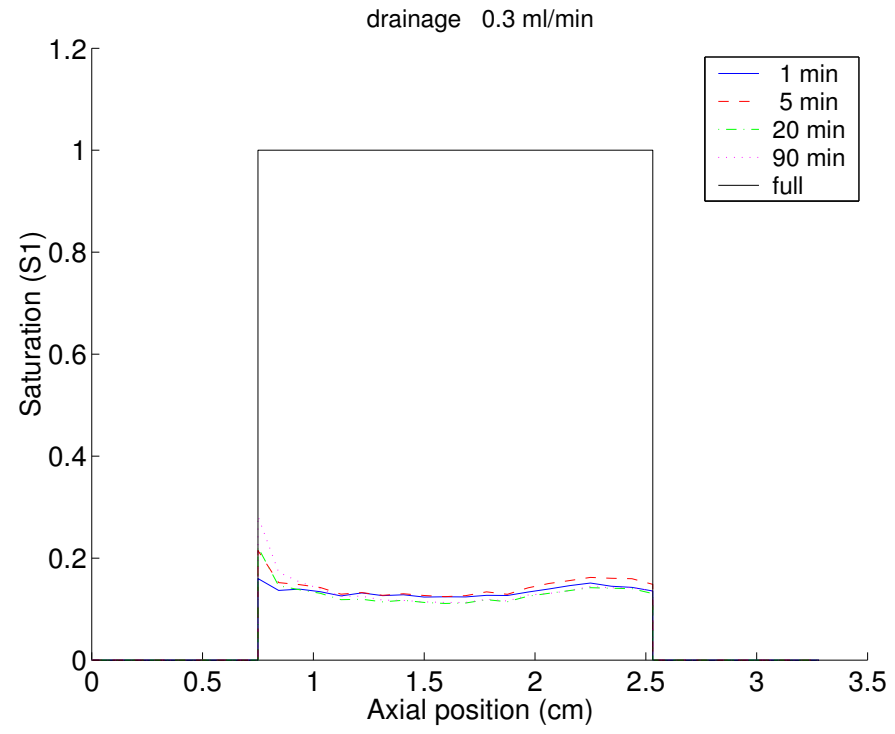

(a) 1D imaging

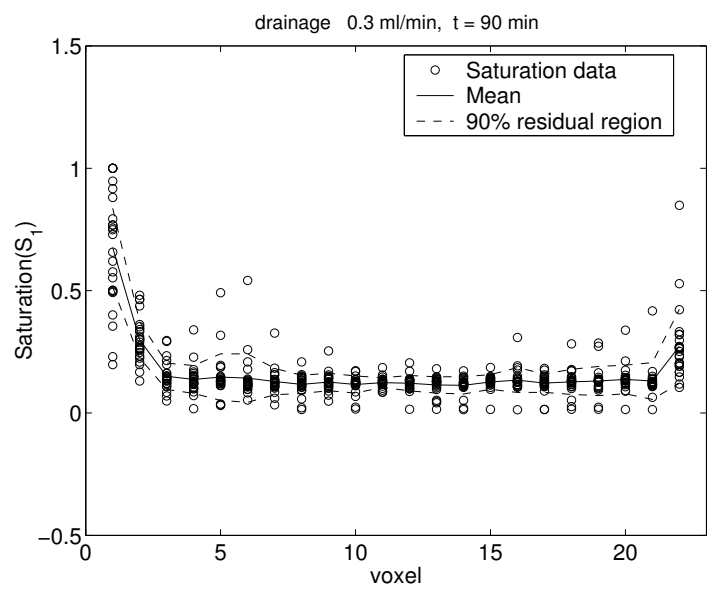

(b) 3D imaging

Figure 50: Saturation of nonwetting phase for 1st cycle of Experiment III $(0.3 \mathrm{ml} / \mathrm{min})$

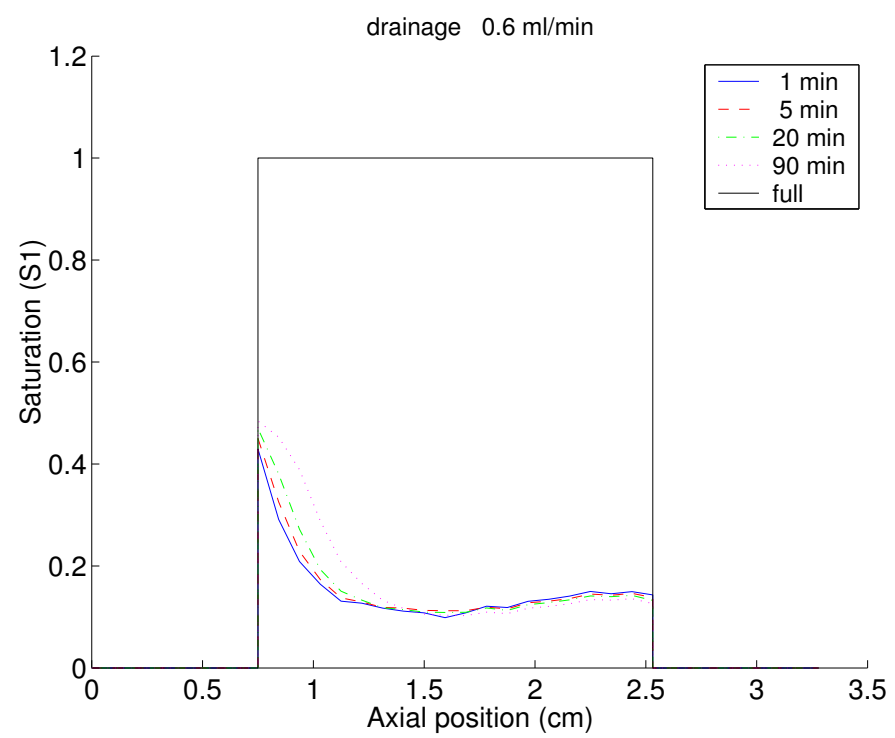

(a) $1 \mathrm{D}$ imaging

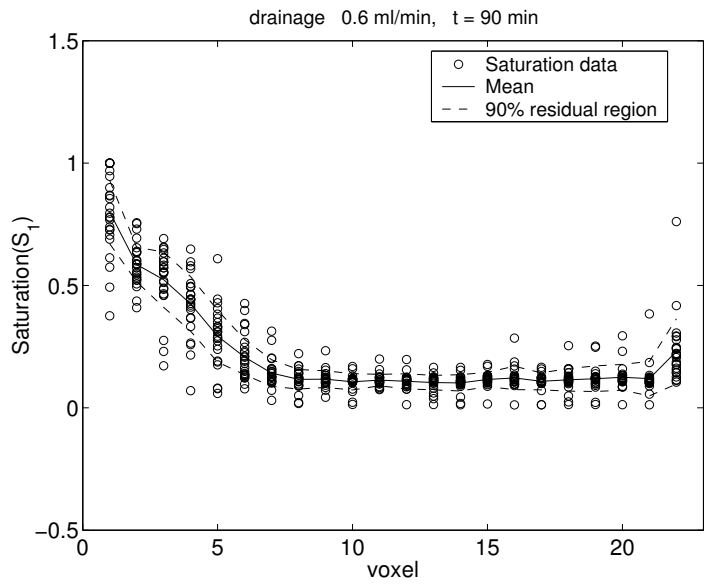

(b) 3D imaging

Figure 51: Saturation of nonwetting phase for 1st cycle of Experiment III $(0.6 \mathrm{ml} / \mathrm{min})$ 


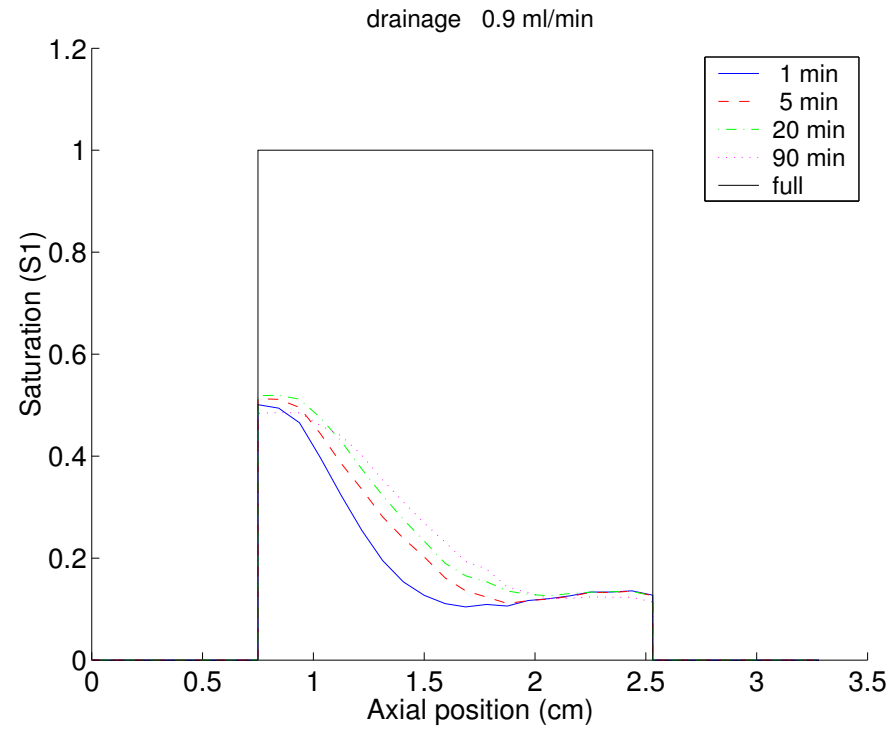

(a) 1D imaging



(b) 3D imaging

Figure 52: Saturation of nonwetting phase for 1st cycle of Experiment III $(0.9 \mathrm{ml} / \mathrm{min})$



(a) $1 \mathrm{D}$ imaging

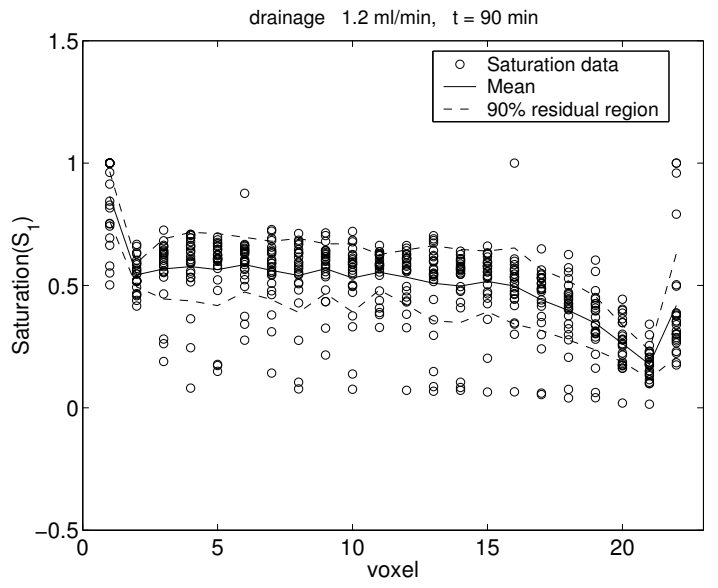

(b) 3D imaging

Figure 53: Saturation of nonwetting phase for 1st cycle of Experiment III $(1.2 \mathrm{ml} / \mathrm{min})$ 


\subsection{Conclusions}

MRI methods for determining intrinsic porosity and permeability and saturation distributions provide powerful means to achieve reliable estimates of multiphase flow properties. We showed that saturation distributions within displacement experiments may not be uniform in the transverse direction, and thus may not be represented accurately by a one-dimensional model of the experiment. We demonstrated that spatial variations in permeability can be a source of significant error in estimating multiphase flow properties, and developed methodology whereby complete spatial distributions for porosity and permeability can be incorporated into the estimation, thus avoided such errors. We provide the means to include both one-dimensional and three-dimensional saturation measurements into the estimation process, and we highly recommend that protocol be used.

Using MRI, we determined that our experiments, and most likely all multiphase displacement experiments, are not modeled accurately with the conventional boundary conditions used to model displacement experiments. We recommend that extensive evaluation of the experimental design and mathematical description is warranted to ensure the displacement experiments are accurately modeled in the estimation process. The validation of estimates of multiphase flow properties using a full, three-dimensional simulation of the displacement experiment is the only way to ensure accurate estimates of the multiphase properties are obtained. 


\section{Prediction of Properties}

Studies of core samples are valuable for these purposes: 1. develop an understanding of the variation of macroscopic properties at the most fundamental scale, 2. develop a better understanding of, and means to mathematically model, more complex processes by observing and accounting for important spatial variations within samples, and 3. develop the means to predict the properties for other media, such as that actually within the reservoir, using other measurements on that media. The methods we developed to obtain spatial distributions of the porosity and permeability at the intrinsic scale, determine saturation distributions, and obtain accurate estimates of multiphase flow properties from displacement experiments provide unprecedented information that can significantly advance the value of experiments with reservoir core samples, and ultimately our ability to characterize and predict reservoir behavior. In this section, we demonstrate the use of intrinsic properties to develop improved methods to predict the flow properties-(absolute) permeability and relative permeability-and to study unstable displacement processes.

\subsection{Introduction}

The ultimate goal for predicting properties is reservoir characterization, i.e., specification of properties required to reliably simulate reservoir behavior. Generally, properties will vary spatially throughout the entire reservoir, and there is little direct sampling available at regions away from the wells. The region around the wells, however, provides the best opportunity to investigate spatial variations in properties, and to map those variations vertically for extrapolation throughout the reservoir, since extensive well-log information is fairly readily available.

Conventional well-logging methods are sensitive (at best) to storage properties-typically the porosity but in some cases the fluid saturations. Of key interest, those, is the permeability, a critical marker for reservoir producibility that can vary by orders of magnitude. There have been numerous attempts to predict permeability from conventional well-logs; however, these are all based on very casual empirical relationships between measured values and permeability. Furthermore, those methods never address the issue of scale associated with permeability.

NMR well-logging provides the best opportunity for predicting permeability from well logs. Generally, NMR response is sensitive to the morphology of permeable media, since it depends on the self-diffusion of molecules through the pore space. The use of NMR relaxation to estimate pore-size distributions illustrates the sensitivity of relaxation to the microscopic structures, which 
control the permeability. Furthermore, NMR diffusion experiments exhibit a profound difference in response for samples with different permeabilities (see figure 8 and associated discussion in Watson and Chang (1997)). In Section 8.2, we describe the basis for permeability prediction from NMR well logs. We then demonstrate how spatially resolved properties can be used to obtain more reliable methods for predicting permeability.

Reliable methods for predicting (absolute) permeability may provide a basis for predicting relative permeability. One such prediction method has been proposed (Chen et al. 1994). Besides its potential use with well-log data, this type of prediction method can provide the means to determine relative permeability functions for unstable displacements. No such method is currently available. In Section 8.3, we demonstrate the use of NMR imaging to predict the wetting-phase relative permeability and to obtain fundamental information for developing improved understanding and mathematical methods for simulating unstable displacements.

\subsection{Prediction of permeability}

Since the permeability is defined within Darcy's law, we must actually determine it by measuring associated fluid states, or functions of those states, and solving the corresponding inverse problem, as discussed in Section 6. However, relationships for predicting the permeability can be developed by establishing, for a given set of samples, a correlation between the permeability and other properties (predictors). By measuring the predictors, the permeability can be calculated using that correlation. In this way, we can predict the permeability without actually having to conduct flow experiments. Conventionally, the sample set corresponds to an apparent value of permeability and other measurements, notably the average porosity and relaxation, for each sample. We investigate the use of a sample set composed of spatially resolved properties. In this way, any correlation among the properties can be better identified by avoiding the averaging, or use of apparent values, over entire samples. Note that our approach has the advantage of providing a much larger sample set, since typically hundreds of values may be available for each sample. Thus, our correlations will be based on thousands of observations, rather than tens, as is conventionally done; this will provide a much more powerful foundation for developing correlation equations.

In the next subsection, we review the correlations that have been developed to predict permeability using well-log observables. In the following subsection, we illustrate our approach. 


\subsubsection{Permeability correlations}

In simple models of porous media, the absolute permeability has been found to be proportional to the square of the surface-to-volume ratio (Scheidegger 1974) and, therefore, proportional to the square of the NMR relaxation times (Kenyon et al. 1988). Seevers (1966) first proposed the estimator $\phi T_{1}^{2}$ for permeability, and later this was revised by Kenyon et al. (1988) who examined more than seventy water-saturated sandstone samples from five oilfield wells in different parts of the world. They proposed, providing physical significance (see section Physical Perspective on Permeability Estimation in Kenyon etal. (1988)), that permeability can be better predicted by $\phi^{4} T_{1}^{2}$.

Further support for this form is provided by the following arguments. Katz and Thompson (1986) observed that the permeability is proportional to the product of the square of the characteristic length associated with the pore space, $l_{c}$, and ratio of the conductivity of the porous sample, $\sigma_{0}$, saturated with fluid of conductivity $\sigma_{w}$ (Katz and Thompson 1986):

$$
K \propto l_{c}^{2} \frac{\sigma_{o}}{\sigma_{w}}
$$

NMR provides an estimate of the characteristic length. As shown in Section 4.2, the relaxation rate associated with a pore is proportional to the effective radius of the pore (see Eqs. 40 and 41). Suppose that we have a characteristic (e.g., average) relaxation time $\tau_{o}$ at fully saturated conditions. Archie's law (Archie 1942) for a fully saturated sample is

$$
\frac{\sigma_{o}}{\sigma_{w}}=\phi^{m}
$$

Consequently, we can write:

$$
K \propto \tau_{o}^{2} \phi^{m}
$$

While a typical value for $m$ in Archie's law is 2, Kenyon et al. (1988) found that an exponent of 4 provided a better correlation of their sample set, as stated previously.

In more recent works (LaTorraca et al. 1993; Kubica 1995; Straley et al. 1997), $T_{2}$ has been used instead of $T_{1}$. The reason for this is that $T_{2}$ is easier to measure in the down-hole environment than $T_{1}$. At the low magnetic field employed by NMR well logging, $T_{2} \approx T_{1}$. At the higher fields, used by Kenyon et al. (1988) as well as by us, $T_{2}$ is strongly affected by diffusion within the magnetic field gradients induced by differences in magnetic susceptibilities of the solid and fluids. Consequently, at the high field only $T_{1}$ provides the intrinsic information about the characteristic pore size that is evidently valuable for permeability correlations. 
The expressions based on pore surface-to-volume ratio have been substantiated by empirical observations on a number of sandstones core samples (Sen et al. 1990; Kubica 1995). In addition to the experimental data, artificial porous media were computationally generated and studied. Dunn et al. $(1998,1999)$ studied periodic porous systems composed of arrays of touching and overlapping spheres, Øren et al. (2002) reconstructed three-dimensional sandstone samples by a processbased reconstruction method, and Hidajat et al. (2002) made use of two-point pore-pore correlation functions to construct porous media. They simulated or calculated the NMR properties and the permeabilities on the artificial porous media and studied their correlations. They showed that the simulated data from the artificial porous media are consistent with the commonly used NMR permeability correlation, $k=a \phi^{b} T^{c}$, and that this expression can be improved by incorporating surface relaxivity and formation factor as additional multipliers with corresponding exponents.

While the studies with artificial porous media are useful to develop better expressions of permeability correlations, the precision of the correlations has understandably been less successful for actual heterogeneous rocks. In actual applications, we might expect bounds not much better than an order of magnitude. We believe a significant problem is the use of the core sample as the fundamental unit associated with the measurements. A single, apparent value of permeability is used for the sample, when in fact it may vary substantially with position. The porosity and the relaxation distributions also vary with position. Consequently, substantial averaging (at best) of the basic properties is occurring prior to application of the correlation equation. If there is a basic relationship among the permeability, porosity, and relaxation that applies at each position within the sample, there is no guarantee that averages among those properties will be as reliably related, or that a relationship among averages would be reliably carried to other length scales. In contrast, we seek to develop correlation relationships on the basis of the intrinsic properties.

\subsubsection{Predictions with intrinsic properties}

Here we develop correlation-based predictions of permeability based on spatially-resolved porosity, relaxation, and permeability distributions. In this way, we expect to avoid significant loss of resolution due to the use of apparent and averaged quantities. Furthermore, we expect to achieve predictions that are statistically more reliable since the number of data will be substantially larger (hundreds or thousands times larger) than the conventional approach.

We focus on the form

$$
k=a \phi^{b}\left(T_{2 l m}\right)^{c},
$$


which seems to be most commonly used. The logarithmic mean of $T_{2}, T_{2 l m}$, is calculated by

$$
T_{2 l m}=\exp \left[\int P(\tau) \ln \tau d \tau\right],
$$

where $P(\tau)$ is the normalized $T_{2}$ relaxation distribution. The parameters $a, b$, and $c$ can be estimated using data collected for a set of samples. Some researchers have sought to specify universal estimates for some of these coefficients. For example, Kenyon has proposed (Kenyon et al. 1988)

$$
k^{a p p}=a\left(\phi^{a v g}\right)^{4}\left(T_{2 l m}^{a v g}\right)^{2}
$$

with $a$ to be estimated from the sample set. We denote the properties with superscript app or ave to emphasize the property is an apparent value, or averaged over the entire sample, respectively.

In this work, we determine $k, \phi$, and $T_{2 l m}$ for each of a number of voxels. Here, the relaxation distribution $P(\tau)$ in Eq. 101 is that of the corresponding voxel. We select parameters by linear regression using the logarithmic transformation of the correlation equation:

$$
\log k=\log a+b \log \phi+c \log T_{2 l m} .
$$

It would be preferable to develop the correlations (i.e., determine the parameters) using spatially resolved properties for each of the samples within a geologically similar unit. However, since we were unable to determine the velocity distributions, and thus resolve permeability distributions, for the reservoir samples, we will demonstrate the process with spatially resolved properties for the single Bentheimer sample.

We consider two different scenarios. For the first, the values developed by (Kenyon et al. 1988) were used for the exponents $(b=4$ and $c=2)$ and the corresponding values of permeability, porosity, and average relaxation for all the voxels were used to estimate the coefficient $a$. For the second, we estimated all three parameters from the data. The results of the linear regressions are presented in Table 13. For the logarithmic permeability data, $y_{i}=\log k_{i}, i=1, \ldots, N$, and their corresponding estimate, $\hat{y}_{i}$, the coefficients of determination, SSE and SST, are respectively defined as

$$
\mathrm{SSE}=\sum\left(\hat{y}_{i}-y_{i}\right)^{2}
$$

and

$$
\mathrm{SST}=\sum\left[\hat{y}_{i}-(1 / N) \sum y_{i}\right]^{2} .
$$

The coefficient of determination, $\mathrm{R}^{2}$, is defined as 1 - SSE/SST, and the root-mean-square-error $(\mathrm{RMSE})$ is $(\mathrm{SSE} / N)^{1 / 2}$. It is observed that the errors between the estimated values and the data 


\begin{tabular}{||c||c|c|c|c|c||}
\hline \hline Scenario & Regression equation & $\mathrm{SSE}$ & $\mathrm{RMSE}$ & $\mathrm{R}^{2}$ & P-value \\
\hline I & $\log k=3.94+4 \log \phi+2 \log T_{2 l m}$ & 140.49 & 0.5875 & - & $<0.001$ \\
\hline II & $\log k=5.57+0.819 \log \phi+0.335 \log T_{2 l m}$ & 33.98 & 0.2897 & 0.1195 & $<0.001$ \\
\hline \hline
\end{tabular}

Table 13: Results of linear regression for the parameters of correlation equation

are reduced by estimating all three parameters instead of estimating one. However, the relatively small $\mathrm{R}^{2}$ value implies that the fitting is not precise for the given correlation equation. The $\mathrm{P}$ value is based on $\chi^{2}$ random distribution and it provides a criterion to test for significance of the regression. The associated null hypothesis is that all the linear regression parameters $(\log a, b$, and $c$ for scenario I and $\log a$ for II) are zero. The very small $\mathrm{P}$-values indicate that the null hypothesis is rejected (i.e., at least one of the parameters are significant) with confidence level more than $99.99 \%$ for both of the scenarios.

In Fig. 54, we plot the measured and predicted values of permeability for the three cases. Perfect predictions will lie along the diagonal. For the first case, there is a clustering around the diagonal, but a number of points are located to the left of the cluster. The clustering about the diagonal is tighter for the second case. While this does confirm the more precise prediction provided by estimating all the coefficients, it would be desirable to have a greater range of permeability values, which would be available by correlating a number of different samples, to fully appreciate the use of intrinsic properties. As is shown in Table 7, the standard deviations of the porosity are relatively larger for the reservoir samples than for the Bentheimer sample, which implies that the reservoir samples generally have greater heterogeneity than the Bentheimer sample. We expect that the correlation would give better precision with the results from the reservoir samples.

It is desired that the predicted values closely match the measured (data) values. This precision is evaluated by calculating the correlation coefficient between predicted and measured permeabilities, while the logarithmic values are used for the linear regressions. The correlation coefficient is calculated as:

$$
\operatorname{Corr}\left(k^{\text {pred }}, k^{\text {data }}\right)=\frac{\operatorname{Cov}\left(k^{\text {pred }}, k^{\text {data }}\right)}{\sqrt{\operatorname{Var}\left(k^{\text {pred }}\right) \operatorname{Var}\left(k^{\text {data }}\right)}},
$$

where Cov and Var are covariance and variance, respectively. The correlation coefficient is a number between 0 and 1 , and the larger the number, the more precise the prediction. In order to insure the significance of the correlation, we performed a randomization test. Here, the order of each of the three data set, $\left\{k_{i}\right\},\left\{\phi_{i}\right\}$, and $\left\{T_{2 l m, i}\right\}$, is randomly permuted so that the correlation among 


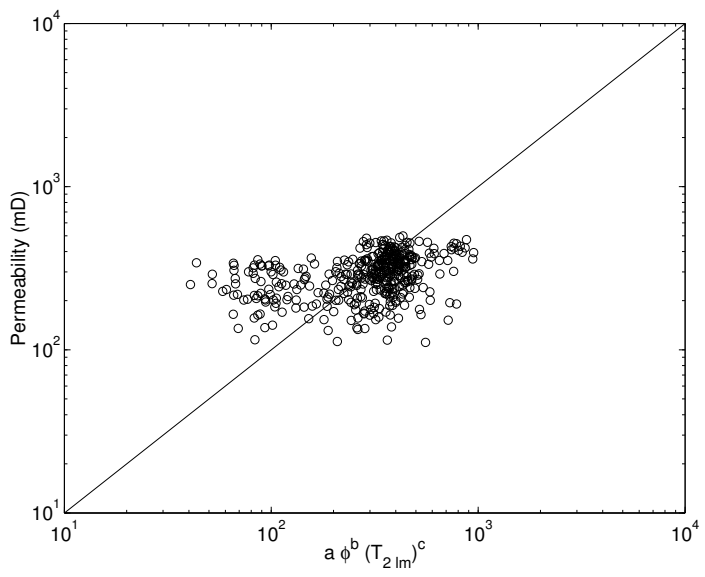

(I) $a=3.94, b=4, c=2$

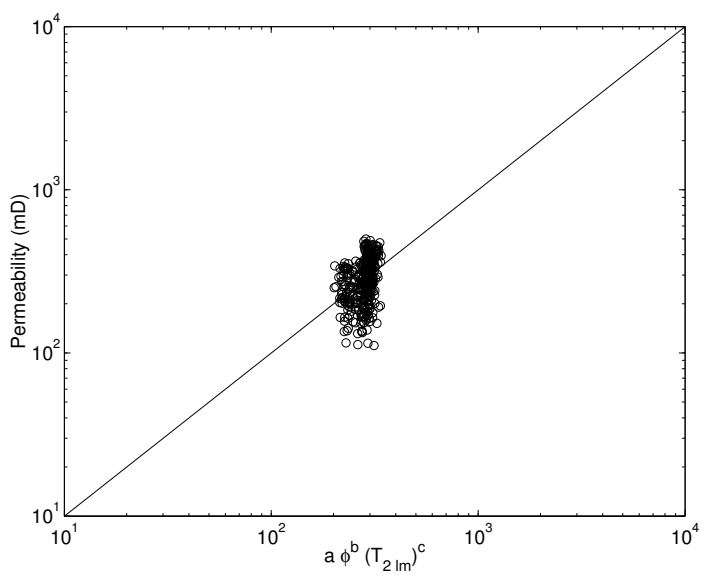

(II) $a=5.57, b=0.819, c=0.335$

Figure 54: Permeability of the voxels in the Bentheimer sample versus its predictor, $a \phi^{b}\left(T_{2 l m}\right)^{c}$. 


\begin{tabular}{||c||c|c||}
\hline \hline Realization & $\operatorname{Corr}\left(k^{\text {pred }}, k^{\text {data }}\right)$ & P-value \\
\hline \hline Original order of data & 0.372 & $<0.0001$ \\
\hline Random permutation 1 & 0.065 & 0.191 \\
\hline Random permutation 2 & 0.076 & 0.124 \\
\hline Random permutation 3 & 0.027 & 0.589 \\
\hline Random permutation 4 & 0.001 & 0.988 \\
\hline Random permutation 5 & 0.073 & 0.140 \\
\hline \hline
\end{tabular}

Table 14: Correlation coefficients for various realizations. The three parameters, $\log a, b$, and $c$ were estimated by linear regression and the corresponding $k^{\text {pred }}$ was calculated.

the randomly ordered values are estimated. The significance of the correlation of the original data is compared to that of the randomly ordered data for which we expect no correlation. We made five such random realizations and performed the same linear regression for the three parameters as we did for scenario II. The correlation coefficient values for original data set and for the random realizations are listed in Table 14.

The table shows that the correlation coefficient of the randomly ordered data is smaller than that of the original data, typically by an order of magnitude. The null hypothesis associated with the $\mathrm{P}$-value is that the correlation coefficient is zero: $\operatorname{Corr}\left(k^{\text {pred }}, k^{\text {data }}\right)=0$. For the original data, the hypothesis is rejected (i.e., the correlation coefficient is not zero, which means there is correlation) with confidence level more than 99.99\%. On the other hand, the hypothesis cannot be rejected (i.e., we cannot avoid the possibility of no correlation) with confidence level down to $90 \%$ for all of the randomly permutated realizations. This test shows that there exists correlation among the properties resolved with respect to the spatial locations within a sample, although the current correlation equation does not a provide precise fit.

It is emphasized that, due to the limited number of samples for which we have resolved the intrinsic permeability, we have not performed an exhaustive search of correlation functions, choosing instead a form based on conventional, whole-sample analyses. The fact that spatial correlation is observed leads us to believe correlation equations can be developed with far greater reliability than current ones, provided the intrinsic permeability can be resolved for the samples of interest. Notably, we recommend evaluation of the inclusion of surface relaxivity, since it provides the scaling between relaxation and pore-size, which has a significant effect on pore size. Our method developed 


\begin{tabular}{||c||c|c|c|c|c||}
\hline \hline Scenario & Correlation & $k^{\text {app,predic }}$ & $\phi^{\text {avg }}$ & $T_{2 l m}$ & $k^{\text {app,data }}$ \\
\hline I & $k^{a p p}=51.4\left(\phi^{\text {avg }}\right)^{4}\left(T_{2 l m}\right)^{2}$ & $290 \mathrm{mD}$ & 0.237 & $42.3 \mathrm{~ms}$ & $267 \mathrm{mD}$ \\
II & $k^{a p p}=262\left(\phi^{\text {avg }}\right)^{0.819}\left(T_{2 l m}\right)^{0.335}$ & $283 \mathrm{mD}$ & & & \\
\hline \hline
\end{tabular}

Table 15: Predicted apparent permeability using the determined correlation equations.

for determining the surface relaxivity (Section 4) can contribute significantly to this endeavor by providing much more reliable and convenient measurements of surface relaxivity than previously available.

It is worthwhile to observe how the apparent property values work with the given correlation parameters. The apparent permeability, $k^{a p p}$, and the bulk porosity, $\phi^{a v g}$, of the Bentheimer sample have been given in previous sections (Sections 5 and 6 ). The logarithmic mean of $T_{2}$ of the whole sample, $T_{2 l m}$ can be calculated from the corresponding $T_{2}$ distribution, which has been presented in Fig. 8. The predictions of the permeability from those apparent values are presented in Table 15. It is notable that the parameter sets I and II are quite different while they both give similar apparent permeability, which is consistent with the apparent permeability data, with the same values of average porosity and relaxation time. This implies that the study of individual voxels in a samples provides new aspects of the correlation that could not be investigated only by using apparent values. 


\subsection{Prediction of relative permeability}

Chen et al. (1994) extended a method for predicting permeability to multiphase situations, providing for the prediction of relative permeability. We review this development.

Replacing the characteristic length with the relaxation time in Eq. 97, we obtain:

$$
k \propto \tau_{o}^{2} \frac{\sigma_{o}}{\sigma_{w}}
$$

Suppose now that we determine an average relaxation time $\tau$ for a sample which has saturation $S_{w}$. The average relaxation rate (inverse of relaxation time) will normally be shorter than for the fully saturated condition since the wetting phase will be preferentially displaced by the nonwetting phase from the larger pores. Applying Eq. 107 for this case, we write:

$$
k\left(S_{w}\right) \propto \tau^{2} \frac{\sigma\left(S_{w}\right)}{\sigma_{w}}
$$

where $\sigma\left(S_{w}\right)$ denotes the conductivity of the partially saturated sample. Taking the ratio of Eqs. 107 and 108, we obtain, for the relative permeability:

$$
k_{r w}=\frac{\tau^{2}}{\tau_{o}^{2}} \frac{\sigma_{o}}{\sigma\left(S_{w}\right)} .
$$

The Archie equation for partially saturated conditions is: $\sigma_{o} / \sigma\left(S_{w}\right)=S_{w}^{n}$, with $n=2$ often approximately valid for many sandstones. Using this in the previous equation, and modifying the saturation term to account for no flow at the irreducible saturation $S_{w i}$, we obtain:

$$
k_{r, w}=\left(\frac{\tau^{2}}{\tau_{0}^{2}}\right)\left(\frac{S_{w}-S_{w i}}{1-S_{w i}}\right)^{n} .
$$

We were unable to directly validate this equation by comparing the predictions with actual measurements of the relative permeability for the same experiment. To do so, it is necessary to measure the relaxation of the wetting phase fluid for different saturation conditions. This requires spatial resolution of the relaxation and saturation distributions with the MRI. While data for onedimensional spatial resolutions of the saturation and transverse relaxation profiles can be acquired relatively rapidly, the measurement of spatially resolved longitudinal relaxation distributions take considerably longer. It would be necessary to use a drainage experiment in order to maintain stable saturation profiles for the imaging time required, since complete, continuous displacement occurs during imbibition experiments. While we use drainage experiments to determine the multiphase flow properties, we do so observing the non-wetting phase while using deuterium-oxide for water (wetting) phase, so that strategy is not suitable. 
We spent considerable effort developing an alternate strategy. In particular, we attempted to use a nitrogen-water system, so that we could observe the water. In the drainage experiment, gas is injected into a sample that is filled with water. However, this is an unstable process, and all methods for determining the relative permeabilities are based on stable displacements. There are two other possibilities that could prove fruitful. The first is to use chemical-shift imaging to distinguish the signals, and the second is to use a hydrogen-free oleic phase. While we have had prior experience with chemical shift imaging (Qin et al. 1995), additional errors are always encountered compared to observing only a single fluid phase, and the method may not work well with some samples. Furthermore, we have only demonstrated it for determining saturation, and have not evaluated its use to reconcile relaxation distributions. An initial investigation for a suitable hydrogen-free oleic liquid did not seem promising: many potential substances were not available in any reasonable quantity, were hazardous, or had unknown solubilities with the oil. We concluded that any alternate approach would require extensive research, with relatively little potential value since the permeability distributions and accurate estimates of multiphase properties for the reservoir samples were not available.

A very interesting potential application for the proposed predictive equation is the estimation of relative permeability for unstable displacements. It is understood that the relative permeability property is valid for both stable and unstable displacements. However, there are no methods for determining relative permeability for unstable situations. Note that simulations of unstable phenomena are stochastic, and thus we are unable to simulate the results of any specific experiment. Consequently, the approach of modeling the experiment and solving the associated inverse problem, used for determining multiphase flow properties with stable displacement experiments, is not directly applicable. The proposed relationship can be used to predict the relative permeability associated with unstable displacements, as illustrated in this section. We also further investigated the dependence of relaxation on saturation (Chen et al. 1994), since that is a critical component for the proposed prediction equation. Note that if relaxation did not depend on saturation, the predictive equation would only provide the assumed power-law function of saturation since the coefficient will remain unity.

\subsubsection{Experiment}

Reservoir sandstone sample R221a, $4.1 \mathrm{~cm}$ in length and $2.5 \mathrm{~cm}$ in diameter, was mounted in a plexiglass holder. (We note here that sample R221 was prepared by removing sections of R221a.) 
A small tube of water was placed on the top of the sample to serve as a reference for intensity calibration in order to determine the porosity and saturation distributions (see Section 5). Initially, the sample is fully saturated with water. The bulk porosity was determined gravimetrically to be 0.25. The sample with the reference standard was placed into a $3.5-\mathrm{cm}$ diameter birdcage RF coil and then inserted into our Bruker BioSpec ${ }^{\circledR}$ 24/30 system (100 MHz) equipped with a \pm 20 Gauss/cm gradient coil inside the $30 \mathrm{~cm}$ magnet bore.

Imaging was performed during conditions of full saturation (Stage 0) and at three successive stages. The stages were established by exposing the inlet to nitrogen at $9.7 \mathrm{KPa}, 19.3 \mathrm{KPa}$, and 29.0 KPa, respectively, while the outlet end was maintained at atmospheric pressure. In each case, after about five minutes, the sample was closed off. The saturation distribution was subsequently monitored. When no changes were noted (typically a half hour), spatially resolved (three-dimensional) CPMG and inversion-pulse recovery experiments were conducted to determine saturation and relaxation distributions.

The parameter values associated with the imaging sequence are shown in Table 16. A $64 \times 8 \times 8$ imaging domain was used. The relatively large value of 64 was used to make sure the reference signal did not overlap the sample signal. Relatively low resolutions were used in the other two directions in order to have manageable experimental times for this exploratory experiment. The position of the sample within the imaging region is shown in Fig. 55. For simplicity, we examine results for a rectangular parallel-piped which is fully within the cylindrical sample as noted in the figure. The range of voxels examined in each of the directions, Posterior-Anterior (P-A), Heat-Foot (H-F), and Left-Right (L-R), are listed in Table 17.

\subsubsection{Results and discussion}

Transverse relaxation distributions corresponding to each voxel for each of the four stages were obtained from the CPMG experiments, as described in Section 3. Using the procedures in Section 5, the porosity was determined from the images for Stage 0, and the saturation distributions for the other three stages. Longitudinal relaxation distributions $P\left(T_{1}\right)$ for each voxel were determined from the spatially-resolved inversion-recovery experiments, and average values of relaxation $\tau$ were calculated for each voxel using Eq. 38.

Average saturation values for the examined region were determined to be $0.9,0.7$, and 0.6 for stages 1,2 , and 3 , respectively. The saturation distribution is shown for several planar regions within the sample. Figs. 56-58 show the saturation distributions corresponding to two successive 


\begin{tabular}{||c|c||}
\hline \hline Spectral Width & $50000 \mathrm{~Hz}$ \\
\hline Spatial Encoding & frequency-phase-phase encoding \\
\hline Slice Orientation & transverse anterior-posterior \\
\hline Excitation Pulse Length & $50 \mu \mathrm{s}$ \\
\hline Refocusing Pulse Length & $5 \mathrm{~s}$ \\
\hline Repetition Time & 32 \\
\hline Number of Echoes (CPMG) & 30 \\
\hline Number of Experiments (Inversion-Recovery) & $3.6 \mathrm{~ms}$ \\
\hline Echo Spacing (CPMG) & $4.8 \mathrm{~cm} \times 4.8 \mathrm{~cm} \times 6.4 \mathrm{~cm}$ \\
\hline Field of View & $64 \times 8 \times 8$ \\
\hline Matrix Size & $0.75 \mathrm{~mm} \times 6 \mathrm{~mm} \times 8 \mathrm{~mm}$ \\
\hline Voxel Size & $30 \mathrm{~min}$ \\
\hline Experiment Duration (Inversion-Recovery) & $150 \mathrm{~min}$ \\
\hline \hline Experiment Duration (CPMG) & \\
\hline
\end{tabular}

Table 16: Parameter values for CPMG imaging and inversion-recovery sequences

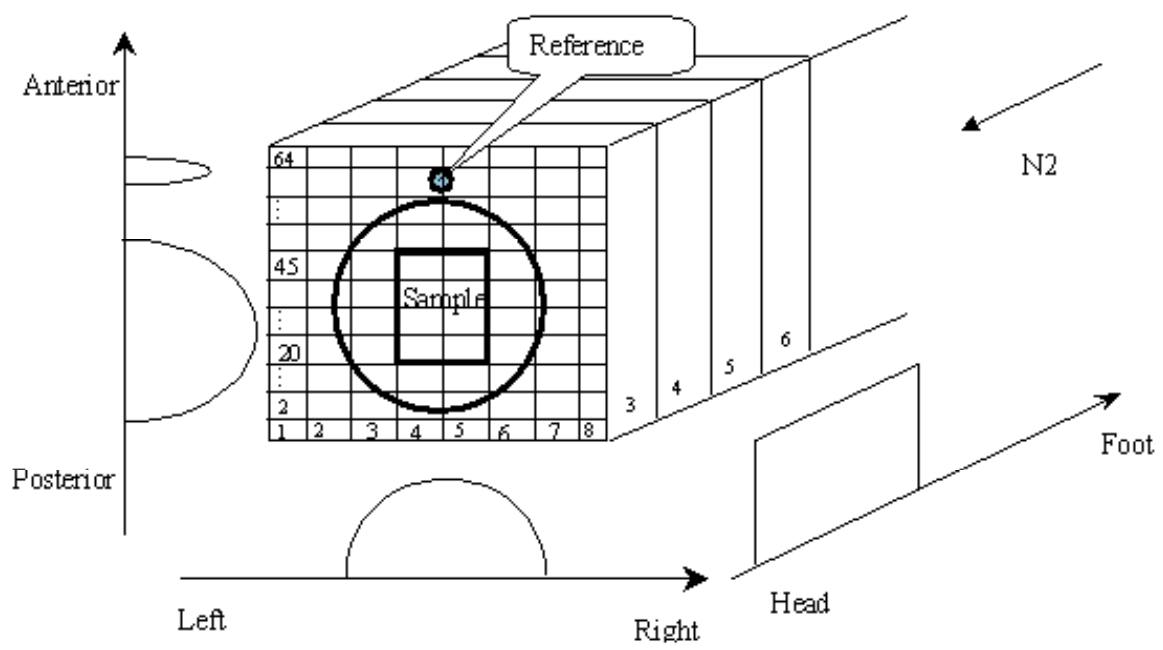

Figure 55: Sample orientation with imaging experiments 


\begin{tabular}{||l|c||}
\hline \hline Sample Limits in P-A Direction & $20-45$ \\
\hline Sample Limits in H-F Direction & $2-7$ \\
\hline Sample Limits in L-R Direction & $4-6$ \\
\hline \hline
\end{tabular}

Table 17: Range of voxels examined
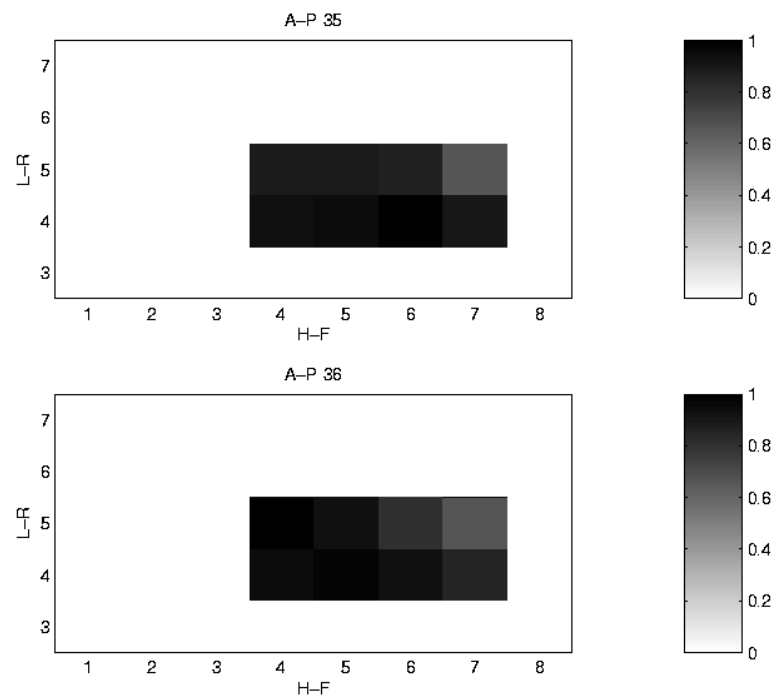

Figure 56: Stage 1 saturation distributions for two successive horizontal planar regions near the center of the sample

horizontal slices (i.e., the H-F-R-F plane) near the center of the sample at each of three stages. Figs. 59-61 show the saturation distributions corresponding to a vertical slice along the axial direction (i.e., the H-F-A-P plane) at each of the stages. All these figures show considerable variations of the saturation, apparently due to the unstable displacements. They also indicated overall decreases in saturation for each of the successive stages.

The large degree of variation in the saturation distributions is further exemplified in Figs. 62-64. All the voxel saturation values are shown, as a function of position along the axial (H-F) direction. Also shown are the average saturation values, and the intervals that contain $95 \%$ of the saturation values. The figures indicate that, as expected, the saturation decreases along the length of the sample for a given stage, and decreasing in each successive stage. It also indicates a very large variation in values at each position along the axial direction.

We illustrate the general behavior of relaxation with saturation with results from two voxels, 

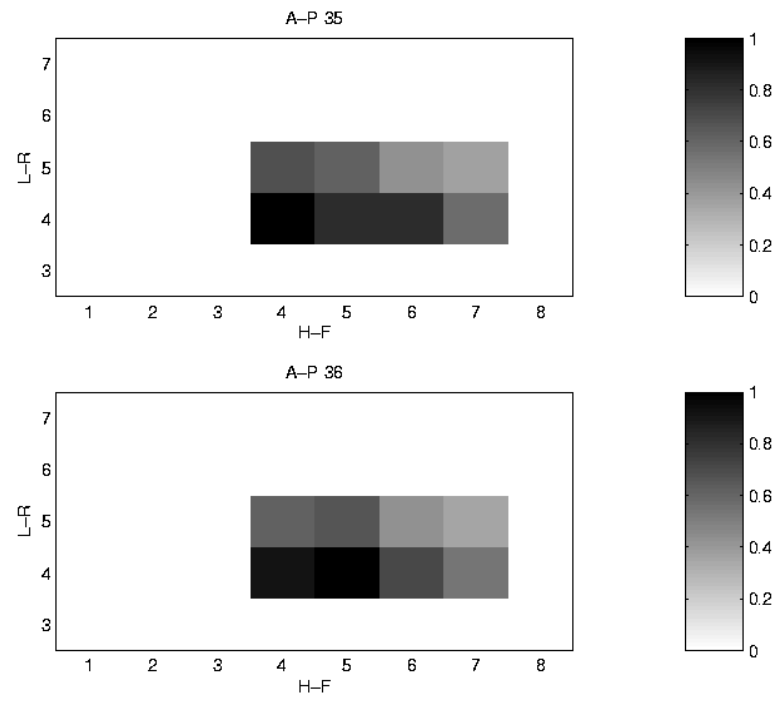

Figure 57: Stage 2 saturation distributions for two successive horizontal planar regions near the center of the sample
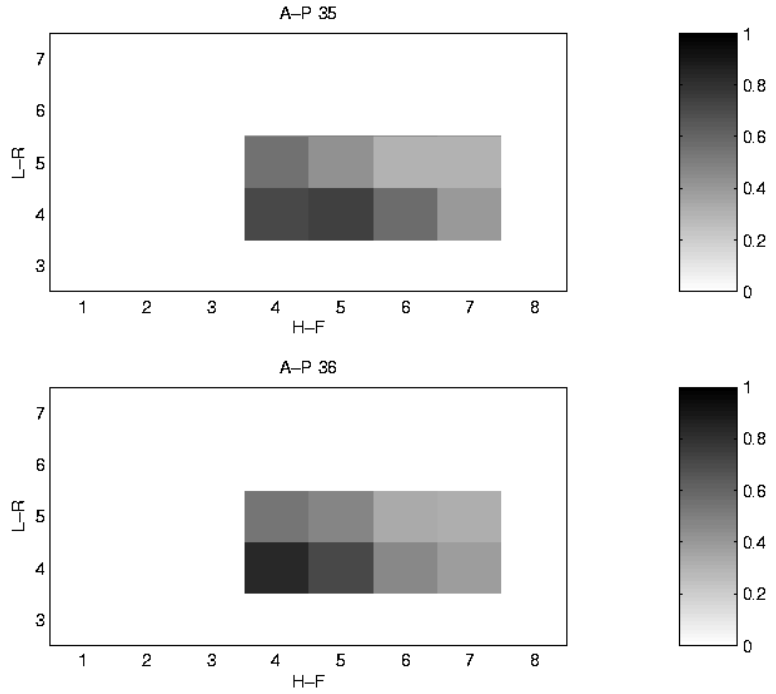

Figure 58: Stage 3 saturation distributions for two successive horizontal planar regions near the center of the sample 

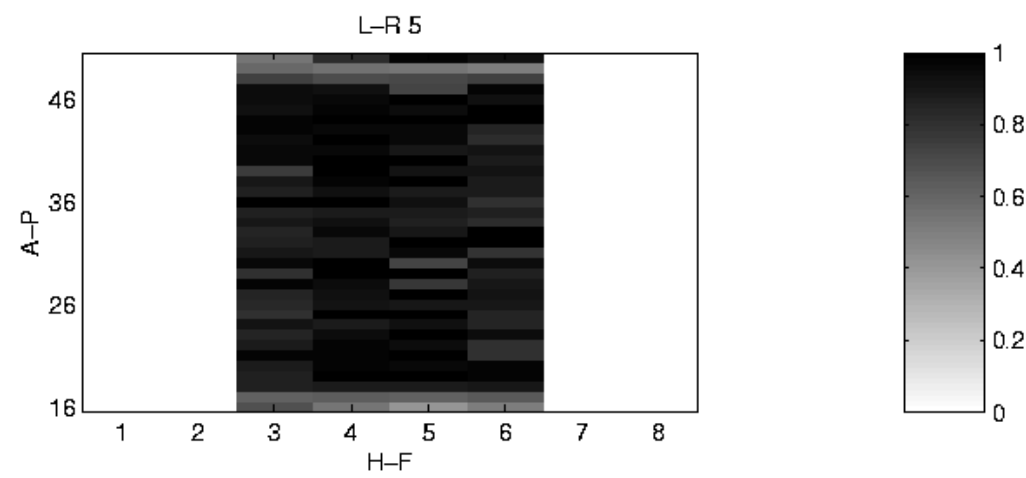

Figure 59: Stage 1 saturation distributions for a horizontal planar region near the center of the sample
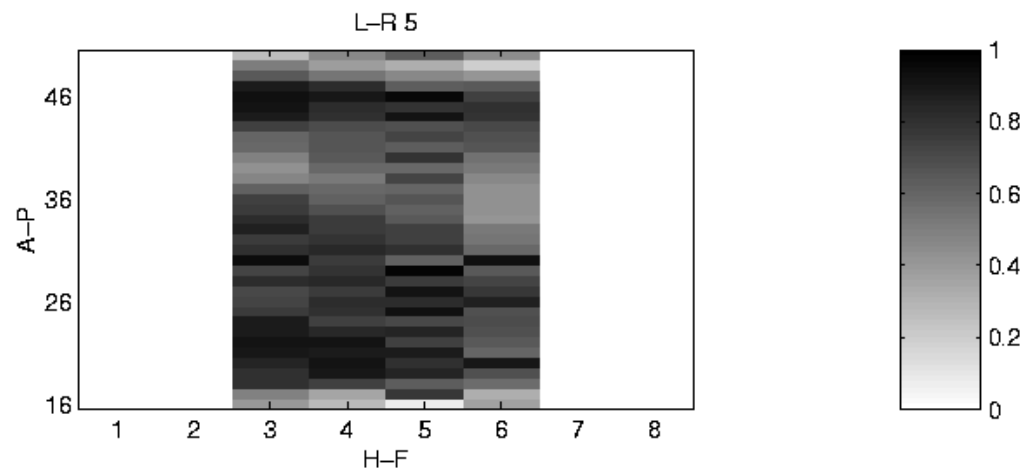

Figure 60: Stage 2 saturation distributions for a horizontal planar region near the center of the sample
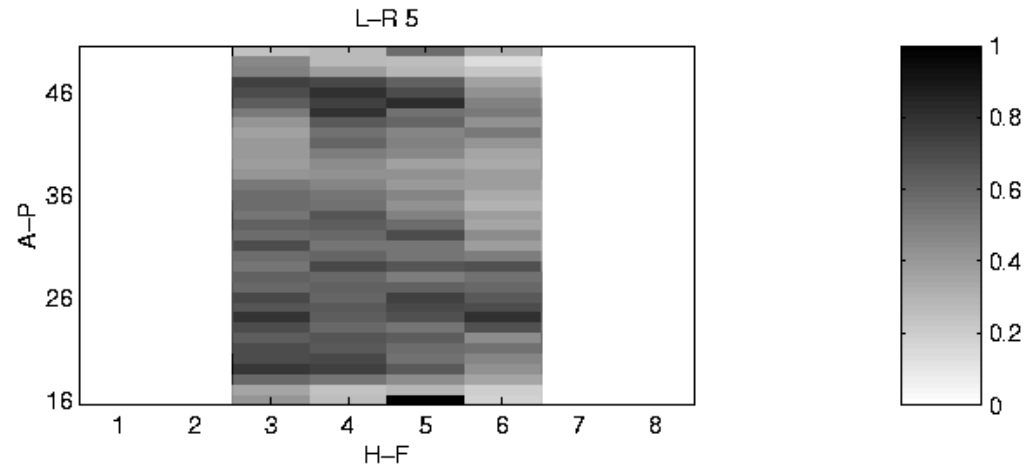

Figure 61: Stage 3 saturation distributions for a horizontal planar region near the center of the sample 


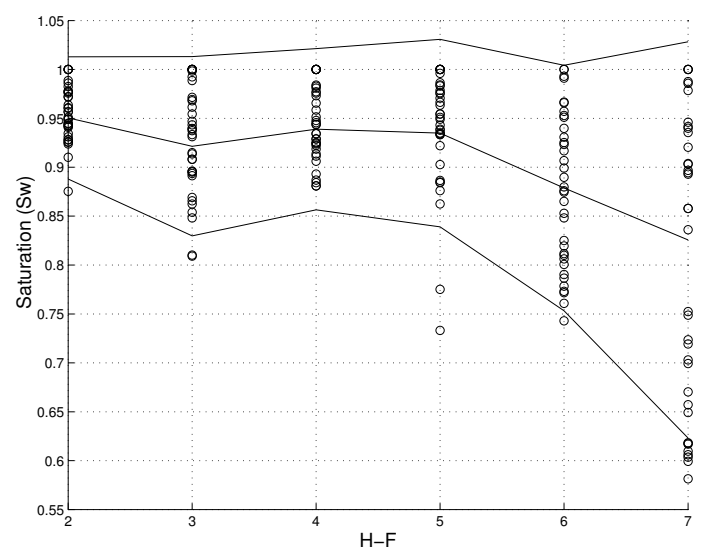

Figure 62: Stage 1 Voxel saturation values along the axial direction

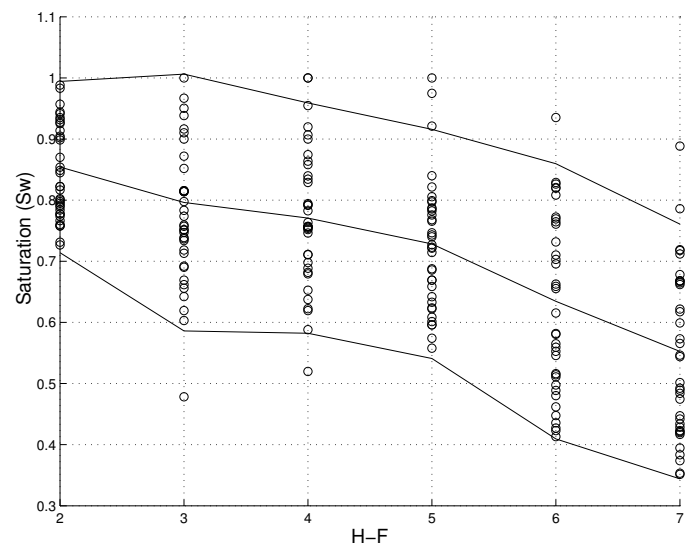

Figure 63: Stage 2 Voxel saturation values along the axial direction

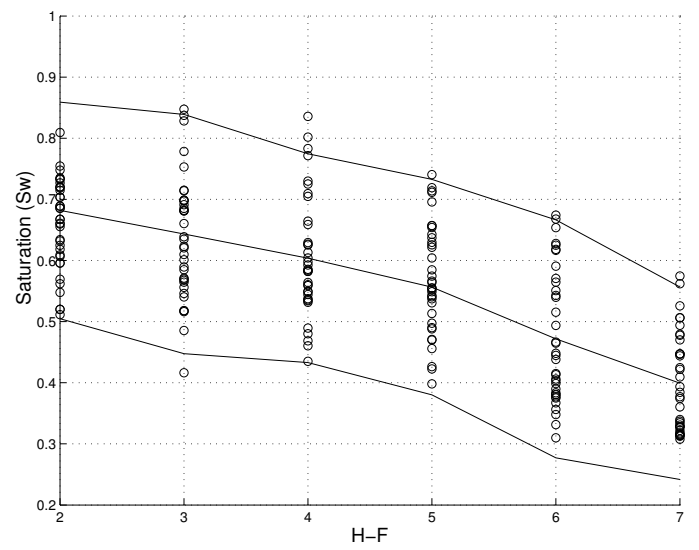

Figure 64: Stage 3 Voxel saturation values along the axial direction 


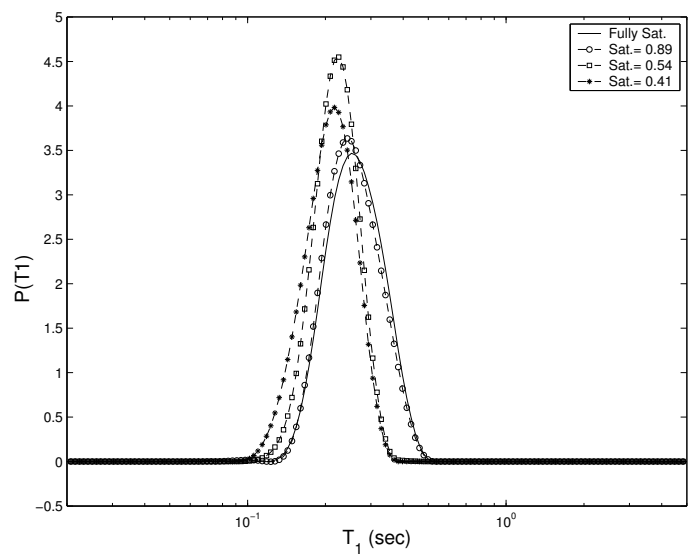

Figure 65: $T_{1}$ distributions for Voxel $(35,7,6)$

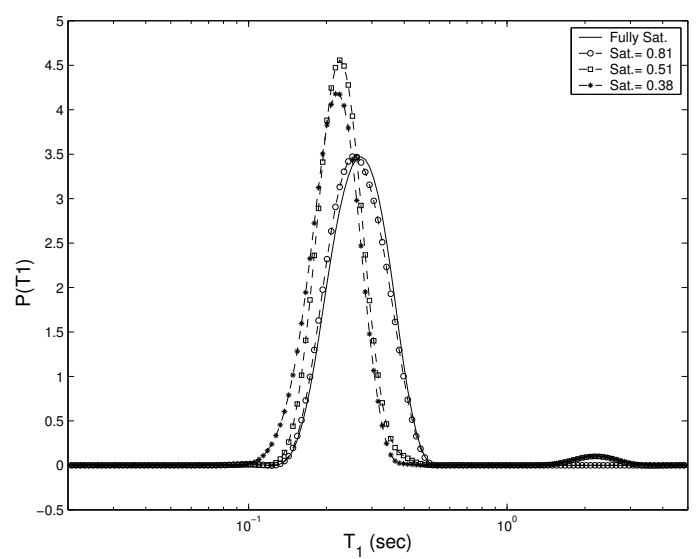

Figure 66: $T_{1}$ distributions for Voxel $(30,6,6)$

whose positions are identified by coordinates (P-A, H-F, L-R), where the indices are the voxel number in each of the posterior-anterior (P-A), head-foot (H-F), and left-right (L-R) directions, respectively (see Fig. 55). The relaxation distributions determined for the two voxels corresponding each of the four stages are shown in Figs. 65 and 66, respectively, and the corresponding average relaxation values are listed in Table 18.

These average relaxation times are plotted as a function of saturation in Fig. 67 . There is a clear trend of the average relaxation with saturation, consistent with that observed by Chen et al. (1994). The differences in the experimental protocols between the cited work and ours are notable. Chen etal. (1994) used averages corresponding to the entire samples, whereas we have resolved spatially both the saturation and relaxation. 


\begin{tabular}{||c|c|c||}
\hline \hline \multicolumn{3}{|c|}{ Voxel (35, 7, 6) } \\
\hline Stage & $\tau(\mathrm{sec})$ & Saturation \\
\hline 0 & 0.25 & 1 \\
\hline 1 & 0.24 & 0.88 \\
\hline 2 & 0.21 & 0.54 \\
\hline 3 & 0.20 & 0.41 \\
\hline \multicolumn{3}{|c||}{ Voxel (30, 6, 6) } \\
\hline Stage & $\tau($ sec $)$ & Saturation \\
\hline 0 & 0.26 & 1 \\
\hline 1 & 0.25 & 0.81 \\
\hline 2 & 0.23 & 0.51 \\
\hline 3 & 0.21 & 0.38 \\
\hline \hline
\end{tabular}

Table 18: Average relaxation and saturation values for two voxels

This dependence of relaxation on saturation was observed consistently for other voxels. This is illustrated by Fig. 68, which contains results for 60 voxels. Results corresponding to each single voxel are connected with lines. Note the the average relaxation associated with the fully saturated conditions $\left(S_{2}=1\right)$ varies considerably. Evidently, this is due in differences in surface relaxivity, which is probably due to variations in chemical composition of the solid material within this sample. This emphasizes the value of this spatial analysis, which negates that effect.

We now demonstrate the prediction of relative permeability. Using data from all the stages, we bin the saturations values by identifying the voxels that fall within specified intervals of saturation with (approximate range of 0.05 saturation units). We determine the relaxation distribution that represents each bin by computing a composite distribution:

$$
\overline{\mathcal{P}}\left(T_{1}\right)=\sum_{i=1}^{N} \mathcal{P}_{i}\left(T_{1}\right),
$$

where $\mathcal{P}_{i}$ represents the (non-normalized) relaxation distribution of the water within the $i$ th voxel in the bin and $N$ is the number of voxels belonging to the bin. The composite distribution, $\overline{\mathcal{P}}\left(T_{1}\right)$, is normalized by

$$
\bar{P}\left(T_{1}\right)=\frac{\overline{\mathcal{P}}\left(T_{1}\right)}{\int_{0}^{\infty} \overline{\mathcal{P}}(\tau) d \tau}
$$

and the average relaxation value is calculated using $\left.T_{1}^{a v g}=\left[\int_{0}^{\infty} \tau^{-1} \bar{P}(\tau) d \tau\right)\right]^{-1}$. We then compute, 


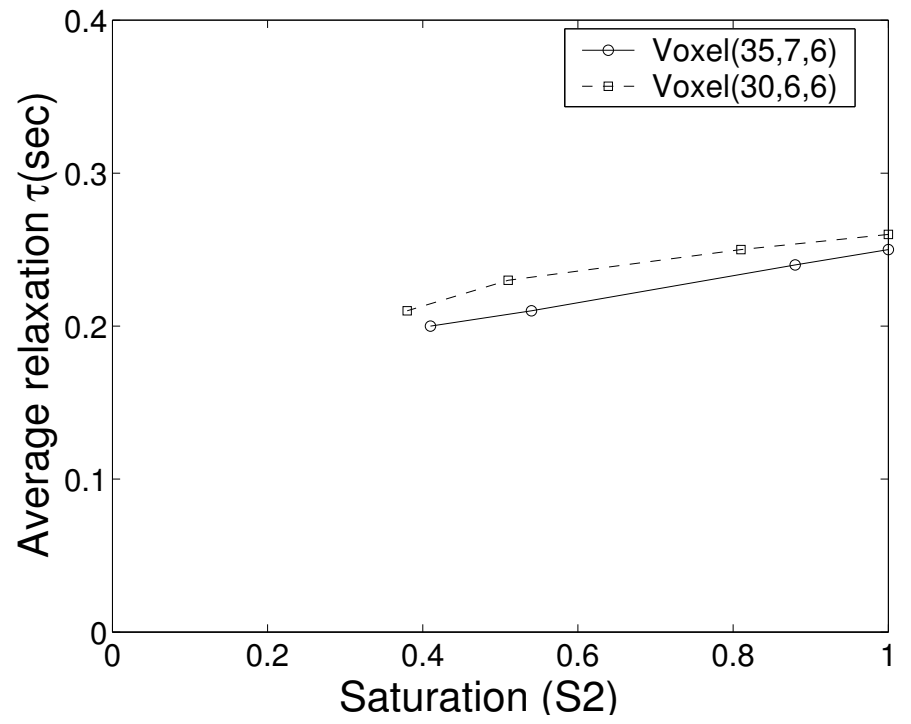

Figure 67: Average relaxation for different saturations for two voxels

using a mass balance, the average saturation for the bin, and calculate the corresponding value of relative permeability using Eq. 110. We estimated the irreducible saturation to be 0.3 by extrapolating a plot of saturation versus inlet gas pressure (Xue 2004). The corresponding predicted relative permeability is shown in Fig. 69 . 


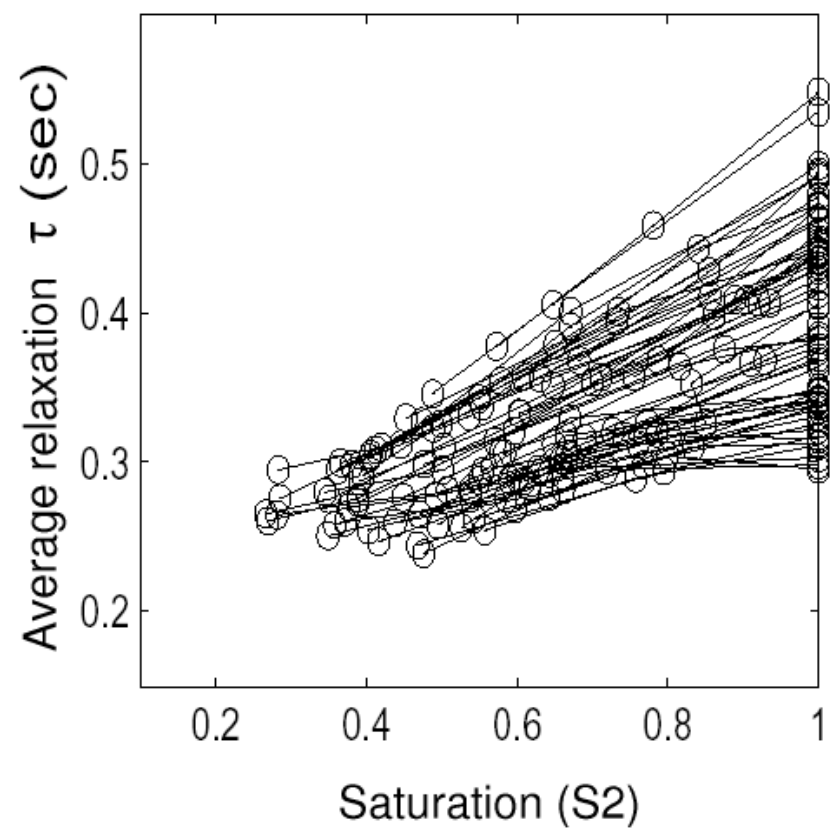

Figure 68: Average relaxation for different saturations for sixty voxels

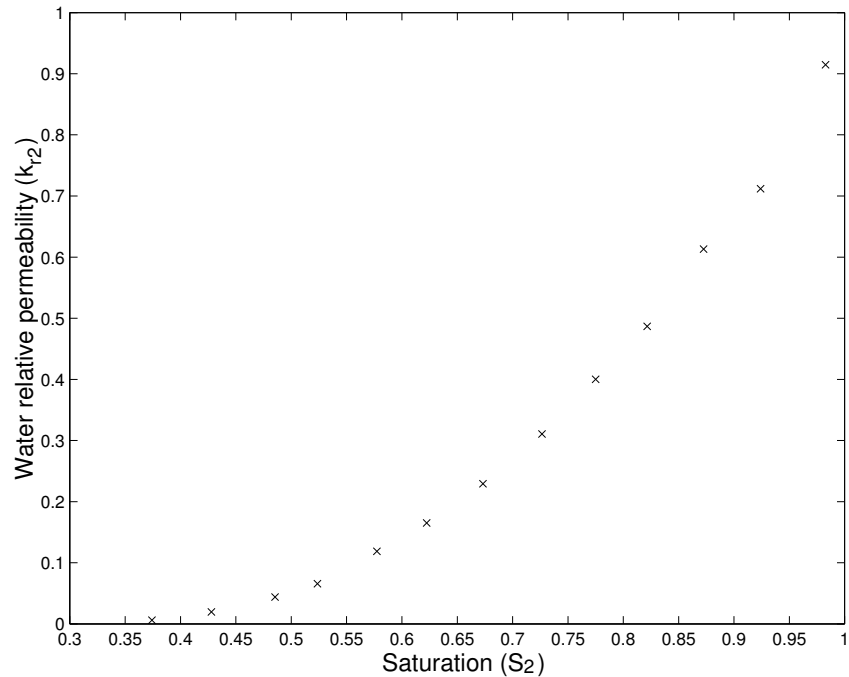

Figure 69: Predicted relative permeability 


\section{Conclusions}

We developed a new, non-parametric based method to estimate relaxation distributions from NMR data. This forms the basis for determining spatially resolved porosity and saturation distributions from MRI data. Using spatially-resolved CPMG imaging, we determine the relaxation distributions at each voxel in order to model the relaxation behavior and estimate the intrinsic magnetization intensity, which is scaled to the porosity or saturation. This method was demonstrated with several Bentheimer and reservoir sandstone samples. The porosity distributions showed substantial variations, clearly indicating the heterogeneous nature of all the samples. Statistical analysis of the associated porosity values indicated that none of the collection of porosity values for any of the samples had a normal distribution.

We developed a pulsed-field-gradient-stimulated-echo method to determine spatially resolved velocity density functions during flow experiments. These were in turn used to determine spatially resolved superficial (average) velocities. This method was successfully applied to Bentheimer samples. However, the relaxation rates associated with the reservoir sandstone samples were sufficiently large that we were unable to resolve velocities. Identification of a suitable fluid that, when saturating the samples, does not exhibit as great relaxation rates as does water would enable resolution of the velocity. Also, greater success is expected with carbonate samples since water saturated samples generally exhibit smaller rates of relaxation than sandstone.

We developed methodology to estimate permeability distributions from the velocity data. We formulate a performance index, based on differences between the measured velocity data and the corresponding values calculated with a mathematical model of the experiment. We then determine the permeability distribution that minimizes the performance index. Our flow experiment is similar to that conventionally used to determine a single value for permeability, in that we flow a fluid through a sample at a specified flow rate and measure the pressure drop. However, we also use MRI to determine the velocity distribution. Our estimation procedure is validated with simulated data and demonstrated using experimental data obtained for a Bentheimer sample.

We have investigated alternative experimental designs that can provide superior estimates of the permeability distribution. In particular, by conducting multiple experiments establishing different flowing patterns, such as by conducting a second experiment in which fluid flows in a direction orthogonal to the first experiment, substantially more accurate estimates can be obtained.

Surface relaxivities are necessary for estimating pore-size distributions from NMR data. These 
properties will also be useful for developing improved correlations to predict permeability. We developed a method to determine surface relaxivities from NMR data that avoids limitations of current methods that require restrictions on the range of time for which data may be used. Furthermore, it does not require estimates of derivatives of data, which can be a significant source for errors.

We advanced the use of full, three-dimensional mathematical models for use in determining multiphase flow properties from displacement experiments. We recommend including intrinsic porosity and permeability, dynamic one-dimensional saturation profiles, and three-dimensional saturation distributions corresponding to steady states within the process to estimate multiphase flow properties. Using MRI, we have determined the boundary conditions conventionally used to model displacement experiments may not accurately match the experiments, and thus recommend future attention to this aspect.

Spatially resolved porosity, permeability, and relaxation distributions will provide much more powerful foundations for developing correlations to predict permeability than the conventional data based on apparent and average values associated with core samples. We evaluated a commonly used correlation equation reported in the literature and found that it did not provide a good correlation for our spatially resolved data for Bentheimer sandstone. However, our analysis showed our data clearly exhibited spatial correlations.

We demonstrated spatial resolution of saturation and relaxation distributions with an unstable displacement process to predict wetting-phase relative permeability. In addition to the potential application to well logging, this example illustrates the experimental methodology to investigate unstable displacement processes and to predict the relative permeability. 


\section{References}

Abragam, A. (1961). The Principles of Nuclear Magnetism. Oxford University Press.

Allen, D. M. (1974). The relationship between variable selection and data augmentation and a method of prediction. Technometrics 16, 125.

Archie, G. E. (1942). The electrical resistivity log as an aid in determining some reservoir characteristics. Trans. Am. Inst. Min. Metall. Pet. Eng. 146, 54.

Ashby, S. F. (1988). Polynomial Preconditioning for Conjugate Gradient Methods. Ph. D. thesis, University of Illinois at Urbana-Champaign.

Beck, J. V. and K. J. Arnold (1977). Parameter Estimation in Engineering and Science. John Wiley and Sons, Inc.

Borgia, G. C., R. J. S. Brown, and P. Fantazzini (1996). Nuclear magnetic resonance relaxivity and surface-to-volume ratio in porous media with a wide distribution of pore sizes. J. Appl. Phys. 79, 3656.

Brownstein, K. R. and C. E. Tarr (1977). Spin-lattice relaxation in a system governed by diffusion. J. Magn. Reson. 26, 17.

Cacuci, D., C. Weber, E. Oblow, and J. Marable (1980). Sensitivity theory for general systems of nonlinear equations. Nucl. Sci. Eng. 75, 88.

Callaghan, P. T. (1995). Pulsed-gradient spin-echo NMR for planar, cylindrical, and spherical pores under conditions of wall relaxation. J. Magn. Reson. A 113, 53.

Caprihan, A. and E. Fukushima (1990). Flow measurements by NMR. Phy. Rep. 198, 195.

Carr, H. Y. and E. M. Purcell (1954). Effects of diffusion on free precession in nuclear magnetic resonance experiments. Phys. Rev. 94, 630.

Chang, C. T., S. Mandava, A. T. Watson, S. Sarkar, and C. M. Edwards (1993). The use of agarose gel for determination of fluid saturations in porous media. Magn. Reson. Imaging 11, 717.

Chang, C. T. P. and A. T. Watson (1999). NMR imaging of flow velocity in porous media. AIChE J. 45, 437 .

Chavent, G., M. Dupuy, and P. Lemonnier (1975). History matching by use of optimal theory. SPE J. February, 74. 
Chen, S., H.-K. K. Liaw, and A. T. Watson (1994). Measurements and analysis of fluid saturation dependent NMR relaxation and linebroadening in porous media. Magn. Reson. Imaging 12, 815.

Chen, W. H., G. R. Galavas, J. H. Seinfeld, and M. L. Wasserman (1974). A new algorithm for automatic history matching. Soc. Pet. Eng. J. 14, 593.

Corana, A., M. Marchesi, C. Martini, and S. Ridella (1987). Minimizing multimodal functions of continuous variables with the simulated annealing algorithm. ACM Transactions on Mathematical Software 13, 262.

Cotts, R. M., M. J. R. Hoch, T. Sun, and J. T. Markert (1989). Pulsed field gradient stimulated echo methods for improved NMR diffusion measurements in heterogeneous systems. J. Magn. Reson. 83, 252.

Coy, A. and P. T. Callaghan (1994). Pulsed gradient spin echo nuclear magnetic resonance for molecules diffusing between partially reflecting rectangular barriers. J. Chem. Phys. 101, 4599.

Craven, P. and G. Wahba (1979). Smoothing noisy data with spline functions: Estimating the correct degree of smoothing by the method of generalized cross-validation. Numer. Math 31, 377.

Dullien, F. A. L. (1992). Porous Media: Fluid Transport and Pore Structure. New York: Academic Press Inc.

Dunn, K.-J., G. A. LaTorraca, and D. J. Bergman (1998). Permeability relation for periodic structures. Magn. Reson. Imaging 16, 553.

Dunn, K.-J., G. A. LaTorraca, and D. J. Bergman (1999). Permeability relation with other petrophysical parameters for periodic porous media. Geophysics 64, 470.

Edwards, C. M., C. T. Chang, and S. Sarkar (1993). The measurement of fluid velocities in porous media. In The Society of Core Analysts Annual Technical Conference Preprints, Paper No. 9310.

Eubank, R. L. (1988). Spline Smoothing and Nonparametric Regression. Marcel Dekker, Inc., New York.

Eubank, R. L. (1999). Nonparametric Regression and Spline Smoothing. Marcel Dekker, Inc., New York. 
Fordham, E. J., S. J. Gibbs, and L. D. Hall (1994). Partially restricted diffusion in a permeable sandstone: Observations by stimulated echo PFG NMR. Magn. Reson. Imaging 12, 279.

Gallegos, D. P., K. Munn, D. M. Smith, and D. L. Stermer (1987). A NMR technique for the analysis of pore structure: Application to materials with well-defined pore structure. J. Coll. Interface Sci. 119, 127.

Garroway, A. N. (1974). Velocity measurements in flowing fluids by NMR. J. Phys. D: Appl. Phys. 7, L159.

Gasser, T., L. Sroka, and C. Jensen-Steinmetz (1986). Residual variance and residual pattern in nonlinear regression. Biometrika 73, 625.

Hansen, P. C. (1992). Analysis of discrete ill-posed problems by means of the L-curve. SIAM Review 34, 561.

Haskell, K. H. and R. J. Hanson (1981). An algorithm for linear least squares problems with equality and nonnegativity constraints. Mathematical Programming 21, 98.

Hidajat, I., M. Singh, J. Cooper, and K. K. Mohanty (2002). Permeability of porous media from simulated NMR response. Transport in Porous Media 48, 225.

Howard, J. J., W. E. Kenyon, and C. Straley (1990). Proton magnetic resonance and pore size variations in reservoir sandstones. SPE 20600, 733.

Howard, J. J. and E. A. Spinler (1993, October 3-6). Nuclear magnetic resonance measurements of wettability and fluid saturations in chalk. In Proceedings of SPE Annual Technical Conference, Houston, TX, pp. SPE paper 26471. SPE Paper 26471.

Hürlimann, M. D., K. G. Helmer, L. L. Latour, and C. H. Sotak (1994). Restricted diffusion in sedimentary rocks. Determination of surface-area-to volume ratio and surface relaxivity. $J$. Magn. Reson. A 111, 169.

Katz, A. J. and A. H. Thompson (1986). Quantitative prediction of permeability in porous rock. Phys. Rev. B 34, 8179 .

Kenyon, W. E., P. I. Day, C. Straley, and J. F. Willemsen (1988). A three-part study of NMR longitudinal relaxation properties of water-saturated sandstones. SPE Formation Evaluation 3, 622 .

Kenyon, W. E., J. J. Howard, A. Sezginer, C. Straley, K. Horkowitz, A. Matteson, and R. Ehrlich (1989, June 11-14). Pore-size distribution and NMR in microporous cherty sandstones. In 
Trans. of the SPWLA 13 Annual Logging Symposium, Volume Paper LL, pp. 1.

Kerig, P. D. and A. T. Watson (1986). Relative permeability estimation from displacement experiments: An error analysis. SPE Res. Eng. 1, 175.

Kirkpatrick, S., C. D. Gelatt, and M. P. Vecchi (1983). Optimization by simulated annealing. Science 220, 671 .

Kleinberg, R. L. (1994). Pore size distributions, pore coupling, and transverse relaxation spectra of porous rocks. Magn. Reson. Imaging 12, 271.

Kleinberg, R. L. (1996). Well logging. In D. M. Grant and R. K. Harris (Eds.), Encyclopedia of Nuclear Magnetic Resonance, Volume 8, pp. 4960. John Wiley \& Sons.

Kleinberg, R. L., W. E. Kenyon, and P. P. Mitra (1994). Mechanism of NMR relaxation of fluids in rock. J. Magn. Reson. A 108, 206.

Kroeker, R. M. and R. M. Henkelman (1986). Analysis of biological NMR relaxation data with continuous distribution of relaxation times. J. Mag. Res. 69, 218.

Kubica, P. (1995). Statistical tests of permeability estimates based on NMR measurements. In SPWLA 36th Annucal Logging Symposium, pp. 1.

Kulkarni, R. N. and A. T. Watson (1997). A robust technique for quantification of NMR imaging data. AIChE J. 43, 2137.

Kulkarni, R. N., A. T. Watson, and J. E. Nordtvedt (1998). Estimation of porous media flow functions using NMR imaging data. Magn. Reson. Imaging 16, 707.

Kulkarni, R. N., A. T. Watson, J. E. Nordtvedt, and A. Sylte (1998). Two-phase flow in porous media: Property identification and model validation. AIChE J. 44, 2337.

LaTorraca, G. A., K.-J. Dunn, and R. J. S. Brown (1993). Predicting permeability from nuclear magnetic resonance and electrical properties measurements. In 1993 Society of Core Analysts Conference, pp. paper 9312.

Latour, L. L., R. L. Kleinberg, P. P. Mitra, and C. H. Sotak (1995). Pore-size distributions and tortuosity in heterogeneous porous media. J. Magn. Reson. A 112, 83.

Latour, L. L., R. L. Kleinberg, and A. J. Sezginer (1992). Nuclear magnetic resonance properties of rocks at elevated temperatures. J. Coll. Interface Sci. 150, 535. 
Latour, L. L., P. P. Mitra, R. L. Kleinberg, and C. H. Sotak (1993). Time-dependent diffusion coefficient of fluids in porous media as a probe of surface-to-volume ratio. J. Magn. Reson. 101, 342.

Lee, T., C. Kravaris, and J. H. Seinfeld (1986). History matching by spline approximation and regularization in single-phase reservoirs. SPE Res. Eng. 1, 521-534.

Liaw, H.-K., R. Kulkarni, S. Chen, and A. T. Watson (1996). Characterization of fluid distributions in porous media by NMR techniques. AIChE J. 42, 538 .

Mandava, S. S., A. T. Watson, and C. M. Edwards (1990). NMR imaging of saturation during immiscible displacements. AIChE J. 36, 1680.

McCall, K. R., D. L. Johnson, and R. A. Guyer (1991). Magnetization evolution in connected pore systems. Phys. Rev. B 44, 7344 .

Meiboom, S. and D. Gill (1959). Modified spin-echo method for measuring nuclear relaxation times. Rev. Sci. Instr. 29, 688.

Mejia, G. M., K. K. Mohanty, and A. T. Watson (1995). Use of in-situ saturation data in estimation of two-phase flow functions in porous media. J. Petroleum Science and Engineering 12, 233.

Mitra, P. P. and P. N. Sen (1992). Effects of microgeometry and surface relaxation on NMR pulsed-field-gradient experiments: Simple pore geometries. Phys. Rev. B 45, 143.

Mitra, P. P., P. N. Sen, L. M. Schwartz, and P. L. Doussal (1992). Diffusion propagator as a probe of the structure of porous media. Phys. Rev. Lett. 68, 3555.

Neuman, S. P. (1980). Adjoint-state finite element equations for parameter estimation. Water Resources Research 16, 331-346.

Øren, P. E., F. Antonsen, H. G. Rueslatten, and S. Bakke (2002). Numerical simulations of NMR responses for improved interpretations of NMR measurement in reservoir rocks. In $2002 S P E$ Technical Conference and Exibition in San Antonio, Texas, pp. SPE 77398.

Packer, K. J., C. Rees, and D. J. Tomlinson (1972). Studies of diffusion and flow by pulsed NMR techniques. Advances in Molecular Relaxation Processes 3, 119.

Petec Software \& Services (2000). CENDRA Verison 1.4 User's Manual. Bergen, Norway.

Phan, J., J. Uh, R. Valestrand, S. Xue, and A. T. Watson (2001, November). NMR characterizations of properties of heterogeneous media. Research Report. U. S. Department of Energy, 
Report Number DE-AC26-99BC15202, Texas A\&M University.

Powell, M. J. D. (1975). Report css 15. Technical report, AERE Harwell.

Qin, F., S. Chen, C. M. Edwards, and A. T. Watson (1995). Determination of two- and threephase fluid saturation distribution during dynamics displacement of experiments in porous media using NMR chemical shift imaging techniques. Proceed. ASME Heat Transfer and Fluids Eng. Division HTD-Vol. 321 and FED-Vol. 233.

Richmond, P. C. (1988). Estimating Multiphase Flow Functions From Displacement Experiments. Ph. D. thesis, Texas A\&M University.

Scheidegger, A. E. (1974). The Physics of Flow through Porous Media. University Toronto Press.

Schumaker, L. L. (1981). Spline Functions: Basic Theory. John Wiley and Sons.

Seevers, D. O. (1966). A nuclear magnetic method for determining the permeability of sandstones. Trans. SPWLA 6, Paper L.

Sen, P. N., C. Straley, W. E. Kenyon, and M. S. Whittingham (1990). Surface-to-volume ratio, charge density, nuclear magnetic relaxation, and permeability in clay-bearing sandstones. Geophysics 55, 61 .

Seto, K. (1999). Miscible Displacement Simulation and Permeability Characterization in Porous Media. Ph. D. thesis, Texas A\&M University.

Slattery, J. C. (1967). Flow of viscoelastic fluids through porous media. AICHEJ 13, 1066.

Snaar, J. E. M. and H. V. As (1993). NMR self-diffusion measurements in a bounded system with loss of magnetization at the walls. J. Magn. Reson. A 102, 318.

Stejskal, E. O. and J. E. Tanner (1965). Spin diffusion measurements: Spin echoes in the presence of a time-dependent field gradient. J. Chem. Phys. 42, 288.

Stephens, M. A. (1976). Asymptotic results for goodness-of-fit statistics with unknown parameters. Annals of Statistics 4, 357.

Stephens, M. A. (1979). Tests of fit for the logistic distribution based on the empirical distribution function. Biometrika 66, 591.

Stone, M. (1974). Cross-validatory choice and assessment of statistical predictions. J. Roy. Stat. Soc. B 36, 111. 
Straley, C., D. Rossini, H. Vinegar, P. Tutunjian, and C. Morriss (1997). Core analysis by lowfield NMR. Log Analyst 38, 43.

Tanner, J. E. and E. O. Stejskal (1968). Restricted self-diffusion of protons in colloidal system by the pulse-gradient, spin-echo method. J. Chem. Phys. 49, 1768.

Timur, A. (1969). Pulsed nuclear magnetic resonance studies of porosity, movable fluid, and permeability of sandstones. J. Petroleum Technology June, 775.

Uh, J., J. Phan, S. Xue, and A. T. Watson (2002, May). NMR characterizations of properties of heterogeneous media. Research Report. U. S. Department of Energy, Report Number DEAC26-99BC15202, Texas A\&M University.

Valestrand, R. (2002). On the Effect of Absolute Permeability Heterogeneity on the Determination of Porous Media Flow Functions. Ph. D. thesis, University of Bergen.

Villalobos, M. and G. Wahba (1987). Inequality-constrained multivariate smoothing splines with application to the estimation of posterior probabilities. J. Am. Stat. Assoc. 82, 239.

Wahba, G. (1978). Smoothing and ill-posed problems. In M. A. Golberg (Ed.), Solution Methods for Integral Equations, pp. 183. Plenum Press.

Watson, A. T. and C. T. P. Chang (1997). Characterizing porous media with NMR methods. Prog. Nucl. Mag. Res. Sp. 31, 343.

Watson, A. T., R. Kulkarni, J.-E. Nordtvedt, A. Sylte, and H. Urkedal (1998). Estimation of porous media flow functions. Meas. Sci. Technol. 9, 898.

Watson, A. T., J. Phan, J. Uh, R. Michalak, and S. Xue (2002, November). NMR characterizations of properties of heterogeneous media. Research Report. U. S. Department of Energy, Report Number DE-AC26-99BC15202, Texas A\&M University.

Watson, A. T., P. C. Richmond, P. D. Kerig, and T. M. Tao (1988). A regression-based method for estimating relative permeabilities from displacement experiments. SPE Res. Eng. 3, 953.

Whitaker, S. (1969). Advances in theory of fluid motion in porous media. Ind. Eng. Chem. 61, 14.

Whittall, K. P., M. J. Bronskill, and R. M. Henkelman (1991). Investigation of analysis techniques for complicated NMR relaxation data. J. Mag. Res. 95, 221.

Whittall, K. P. and A. L. MacKay (1989). Quantitative interpretation of NMR relaxation data. J. Mag. Res. 84, 134. 
Withjack, E. M. (1988). Computed tomography for rock-property-determination and fluid-flow visualization. SPE Formation Evaluation 3, 696.

Xue, S. (2004). Towards Improved Methods for Determining Porous Media Multiphase Flow Functions. Ph. D. thesis, Texas A\&M University.

Yang, P.-H. and A. T. Watson (1988). Automatic history matching with variable metric methods. SPE Res. Eng. 3, 995-1001. 


\section{List of Acronyms and Abbreviations}

$\begin{array}{ll}\text { BFGS } & \text { Broyden-Fletcher-Goldfarb-Shanno } \\ \text { CPMG } & \text { Carr-Purcell-Meiboom-Gill } \\ \text { CT } & \text { Computed Tomography } \\ \text { CV } & \text { Cross Validation } \\ \text { GCV } & \text { Generalized Cross Validation } \\ \text { ISD } & \text { Integral Square Difference } \\ \text { MRI } & \text { Magnetic Resonance Imaging } \\ \text { MSE } & \text { Mean Square Error } \\ \text { NMR } & \text { Nuclear Magnetic Resonance } \\ \text { PRESS } & \text { Predicted Residual Sum of Squares } \\ \text { PFGSTE } & \text { Pulsed Field Gradient Stimulated Echo } \\ \text { RF } & \text { Radio Frequency } \\ \text { RMSE } & \text { Root Mean Square Error } \\ \text { SSE } & \text { Sum of Squares Error } \\ \text { SST } & \text { Sum of Squares Total } \\ \text { STE } & \text { Stimulate Echo } \\ \text { TE } & \text { Echo Time } \\ \text { TM } & \text { Mixing Time } \\ \text { UBPR } & \text { Unbiased Prediciton Risk } \\ \text { UBR } & \text { Unbiased Risk } \\ & \end{array}$




\section{Appendix 1. Linearly constrained quadratic minimization problem}

In this section, the minimization problem with equality constraints is converted to unconstrained problem to obtain an expression for the Hat matrix. Consider a linearly constrained, quadratic minimization problem:

$$
\min _{\mathbf{c}} J(\mathbf{c})=\|\mathbf{Y}-\mathbf{A} \mathbf{c}\|^{2}
$$

subject to

$$
\mathrm{Gc}=\mathbf{0} .
$$

Here, $\mathbf{A}$ is $n \times n_{s}$ matrix, and $\mathbf{Y}$ and $\mathbf{c}$ are respectively $n \times 1$ and $n_{s} \times 1$ column matrices (or vectors).

Suppose all dependent constraints are eliminated and $\mathbf{G}$ is composed of $u$ independent constraints. In other words, $\mathbf{G}$ is $u \times n_{s}$ matrix with full row rank $\left(u<n_{s}\right)$. Then, a $\mathbf{L Q}$ factorization reduces $\mathbf{G}$ into $\mathbf{L}$, an $u \times u$ lower diagonal matrix, and an $n_{s} \times n_{s}$ orthogonal matrix $\mathbf{Q}$ so that

$$
\mathbf{G}=[\mathbf{L}: \mathbf{0}] \mathbf{Q}
$$

Using the orthonormal properties of Q, Eq. 115 is equivalently expressed as

$$
\mathbf{G Q}^{T}=\mathbf{G}\left[\mathbf{Q}_{1}^{T}: \mathbf{Q}_{2}^{T}\right]=\left[\begin{array}{lll}
\mathbf{L}: \mathbf{0} & ]
\end{array}\right.
$$

Here, $\mathbf{Q}^{T}$ consists of the first $u$ columns, $\mathbf{Q}_{1}^{T}$, and the other $n_{s}-u$ columns, $\mathbf{Q}_{2}^{T}$. Since the orthonormal columns of $\mathbf{Q}^{T}$ form a basis of $n_{s}$-dimensional vector space, the vector $\mathbf{c}$ can be expressed as

$$
\mathbf{c}=\mathbf{Q}_{\mathbf{1}}^{T} \mathbf{c}_{1}+\mathbf{Q}_{\mathbf{2}}^{T} \mathbf{c}_{2},
$$

where $\mathbf{c}_{1}$ and $\mathbf{c}_{2}$ are $u \times 1$ and $\left(n_{s}-u\right) \times 1$ matrices, respectively. Combining Eqs. 114 and 117 gives

$$
\mathbf{G Q}_{\mathbf{1}}^{T} \mathbf{c}_{1}+\mathbf{G Q}_{\mathbf{2}}^{T} \mathbf{c}_{2}=\mathbf{0}
$$

Using $\mathbf{G Q}_{1}^{T}=\mathbf{L}$ and $\mathbf{G Q}_{2}^{T}=\mathbf{0}$ from Eq. 116, Eq. 118 becomes

$$
\mathbf{L c}_{1}=\mathbf{0}
$$

i.e., $\mathbf{c}_{\mathbf{1}}=\mathbf{0}$. This results in

$$
\mathbf{c}=\mathbf{Q}_{2}^{T} \mathbf{c}_{2} .
$$


The minimization problem with constraints (Eqs. 113 and 114) is now equivalently written as

$$
\min _{\mathbf{c}_{\mathbf{2}}} J\left(\mathbf{c}_{\mathbf{2}}\right)=\left\|\mathbf{Y}-\mathbf{A} \mathbf{Q}_{2}^{T} \mathbf{c}_{2}\right\|^{2}
$$

without additional equality constraints. Equation 121 implies that the degree of the freedom of the performance index is reduced from $n_{s}$ of $\mathbf{c}$ to $n_{s}-u$ of $\mathbf{c}_{2}$ due to the equality constraints. This equation can be rewritten in terms of $\mathbf{c}$ instead of $\mathbf{c}_{2}$. Since $\mathbf{Q}_{2}^{T}$ composed of orthonormal columns,

$$
\mathbf{Q}_{2} \mathbf{c}=\mathbf{Q}_{2} \mathbf{Q}_{2}^{T} \mathbf{c}_{2}=\mathbf{c}_{2}
$$

Therefore, the minimization problem is rewritten as

$$
\min _{\mathbf{c}} J(\mathbf{c})=\left\|\mathbf{Y}-\mathbf{A} \mathbf{Q}_{2}^{T} \mathbf{Q}_{2} \mathbf{c}\right\|^{2}
$$

The above derivation is about the performance index without regurization term. However, that can be easily applied for the one with regularization term (Eq. 16). Noting that the vector norm, $\|\cdot\|$, is defined as

$$
\|\mathbf{b}\|^{2}=\mathbf{b}^{T} \mathbf{b}
$$

the performance index with regularization term (Eq. 16) can be written as

$$
J=\left\|\left(\begin{array}{c}
\mathbf{Y}^{\text {data }} \\
\mathbf{0}
\end{array}\right)-\left(\begin{array}{c}
\mathbf{A} \\
\sqrt{n \lambda} \mathbf{M}
\end{array}\right) \mathbf{c}\right\|^{2} .
$$

In analogy with the above derivation (Eqs. 113 and 123), Eq. 125 becomes

$$
J=\left\|\left(\begin{array}{c}
\mathbf{Y}^{\text {data }} \\
\mathbf{0}
\end{array}\right)-\left(\begin{array}{c}
\mathbf{A} \mathbf{Q}_{2}^{T} \mathbf{Q}_{2} \\
\sqrt{n \lambda} \mathbf{M} \mathbf{Q}_{2}^{T} \mathbf{Q}_{2}
\end{array}\right) \mathbf{c}\right\|^{2}
$$

when it is constrained by Eq. 114. Therefore, Eq. 16 with the equality constraints is equivalently written as

$$
\min _{\mathbf{c}} J=\left\|\mathbf{Y}^{\text {data }}-\tilde{\mathbf{A}} \mathbf{c}\right\|^{2}+n \lambda\|\tilde{\mathbf{M}} \mathbf{c}\|^{2},
$$

where $\tilde{\mathbf{A}}=\mathbf{A} \mathbf{Q}_{2}^{T} \mathbf{Q}_{2}$ and $\tilde{\mathbf{M}}=\mathbf{M} \mathbf{Q}_{2}^{T} \mathbf{Q}_{2}$. The corresponding hat matrix is given by

$$
\mathbf{H}(\lambda)=\tilde{\mathbf{A}}\left[\tilde{\mathbf{A}}^{T} \tilde{\mathbf{A}}+n \lambda \tilde{\mathbf{M}}^{T} \tilde{\mathbf{M}}\right]^{-1} \tilde{\mathbf{A}}^{T}
$$




\section{Appendix 2. NMR relaxation distributions}

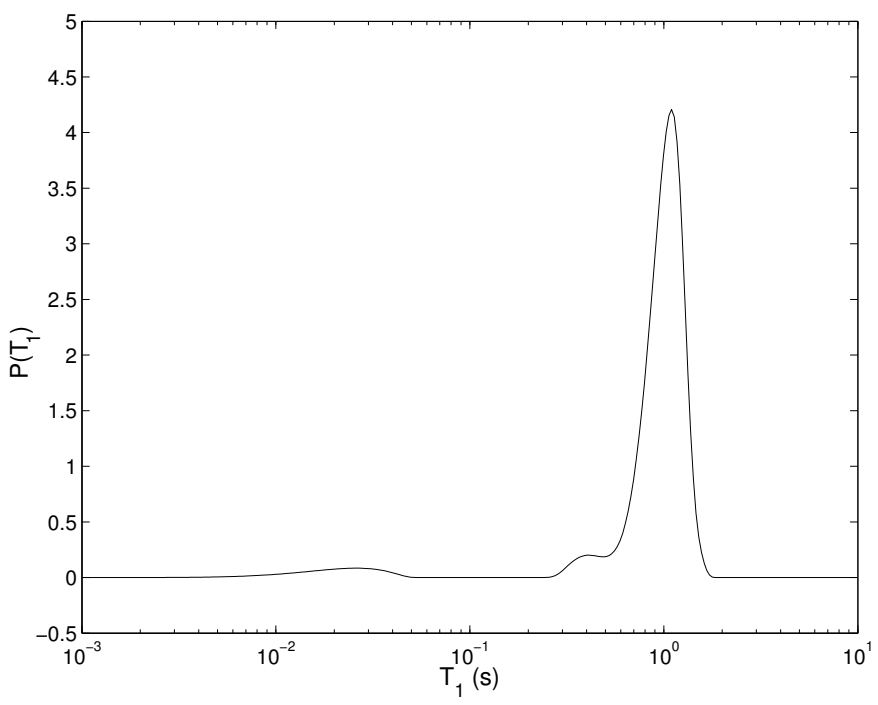

(a) $T_{1}$

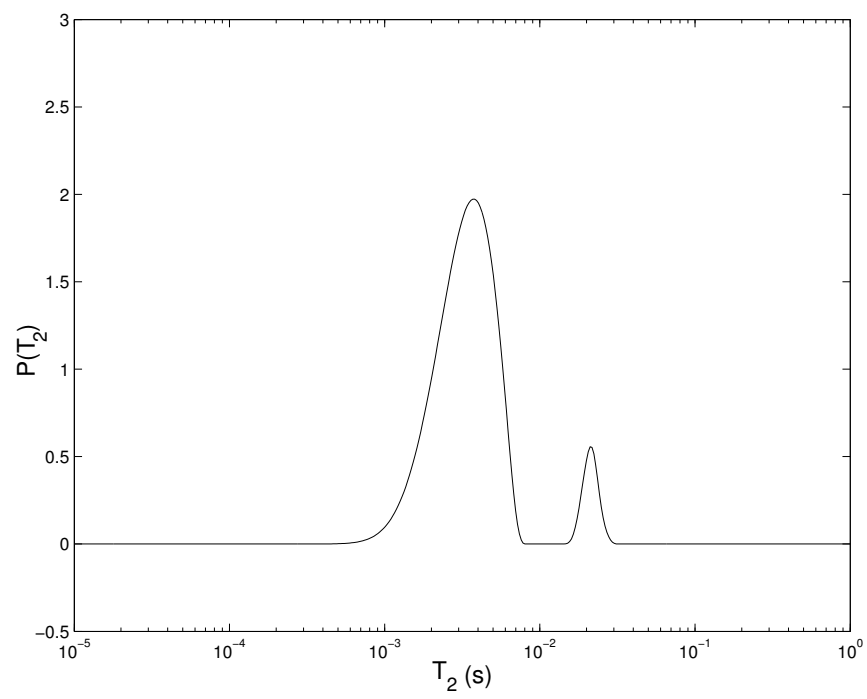

(b) $T_{2}$

Figure 70: NMR relaxation distributions of R138 


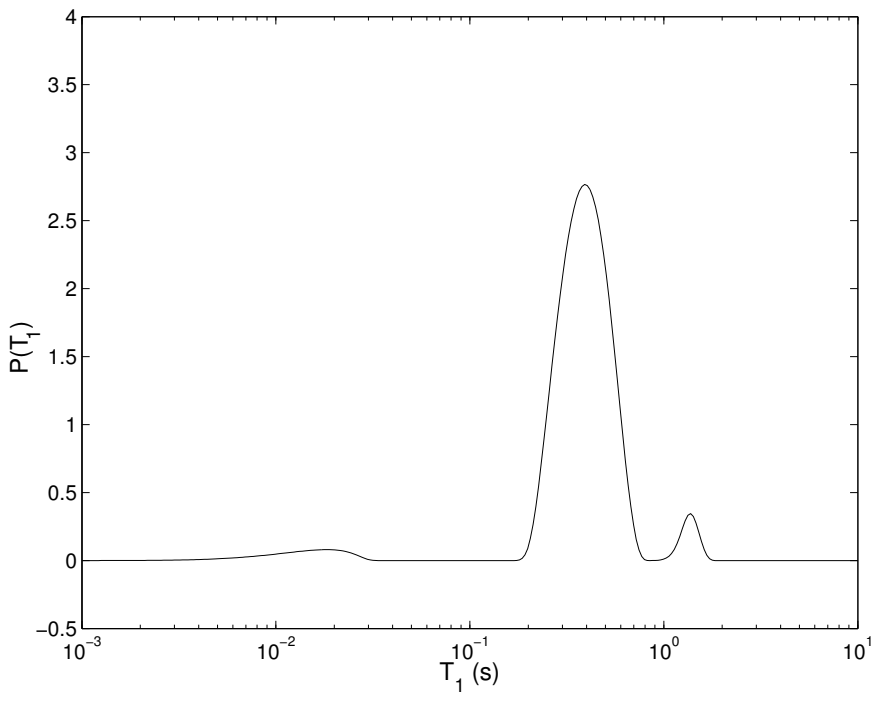

(a) $T_{1}$

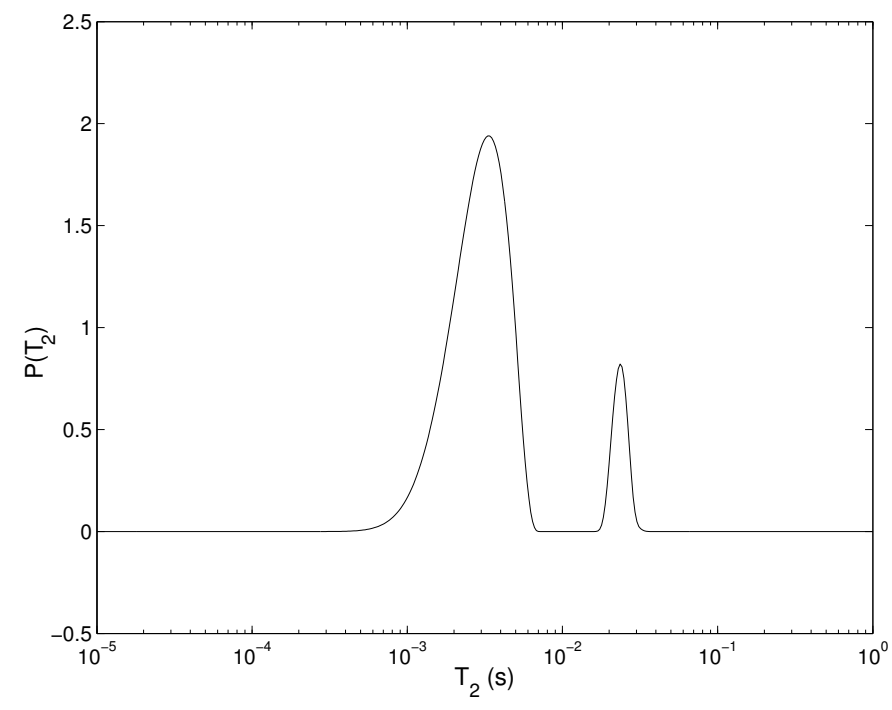

(b) $T_{2}$

Figure 71: NMR relaxation distributions of R145 


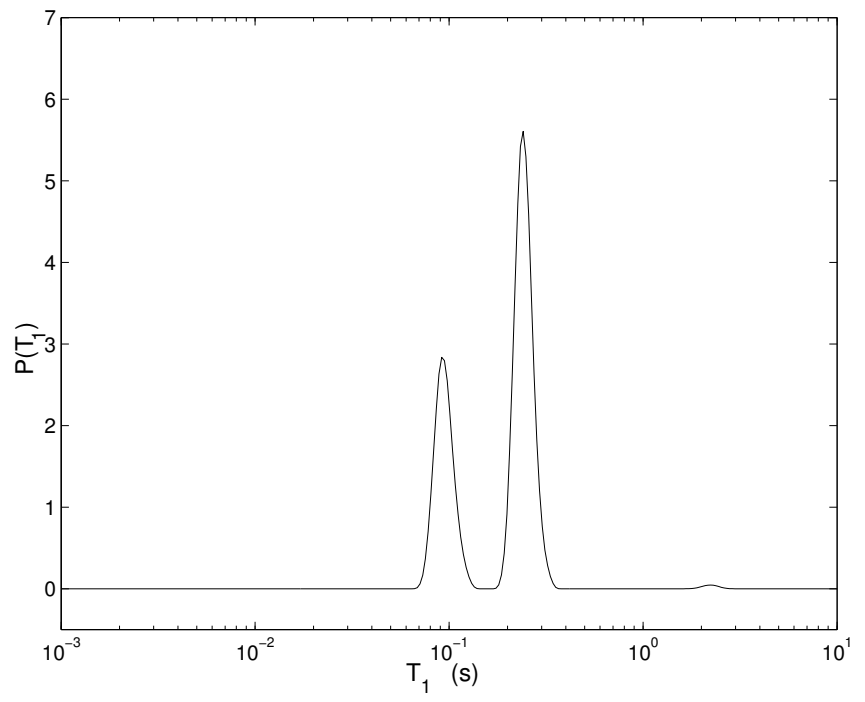

(a) $T_{1}$

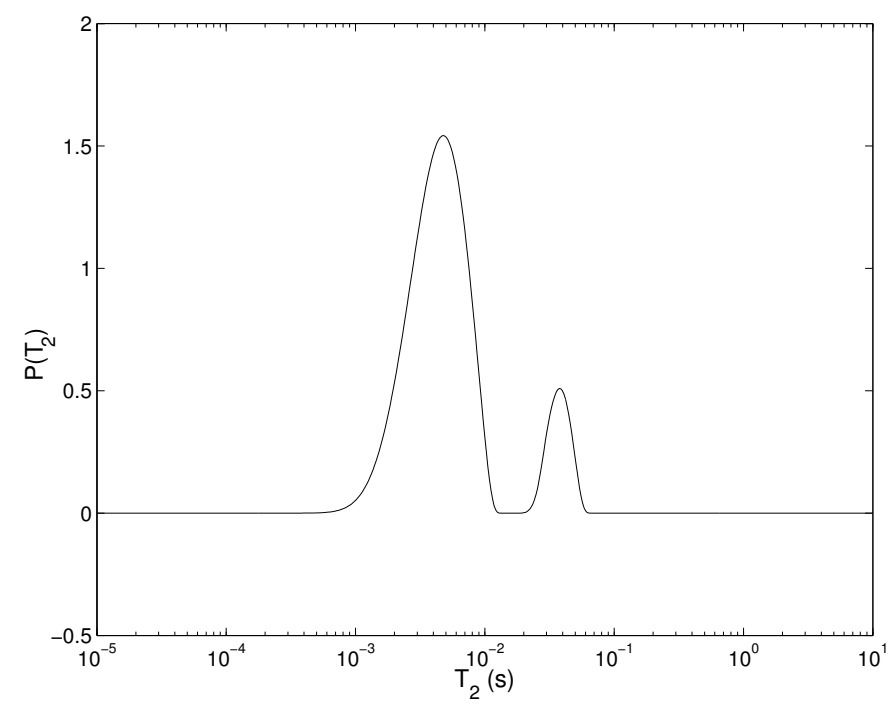

(b) $T_{2}$

Figure 72: NMR relaxation distributions of R221 


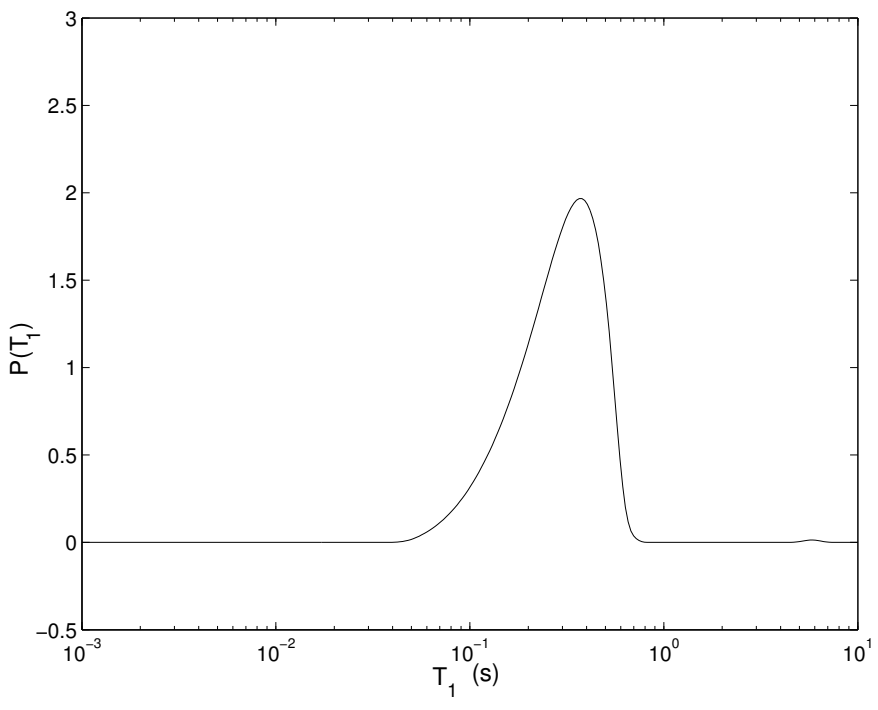

(a) $T_{1}$

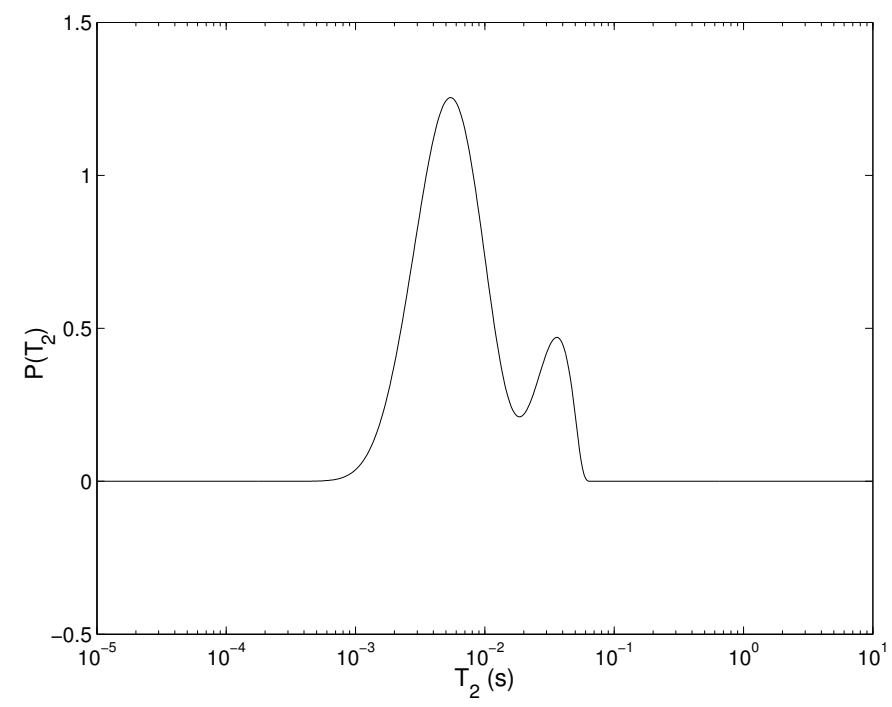

(b) $T_{2}$

Figure 73: NMR relaxation distributions of R237 


\section{Appendix 3. Porosity distributions}
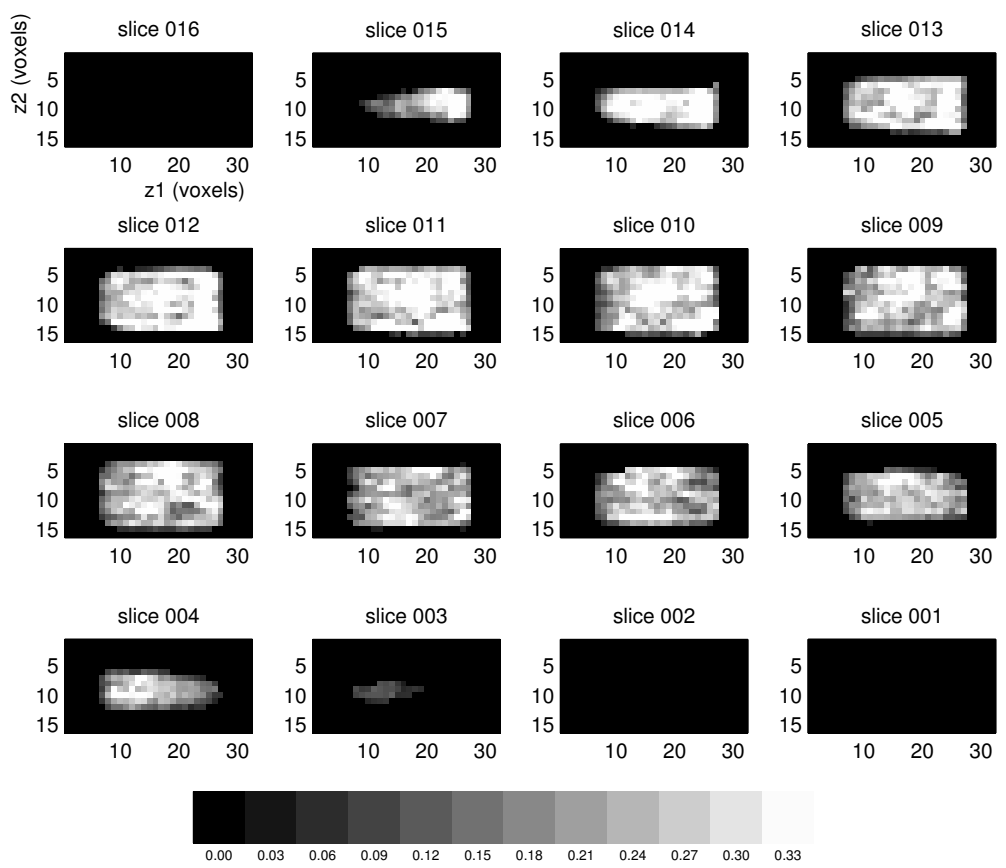

(a) Three-dimensional porosity image in gray scale

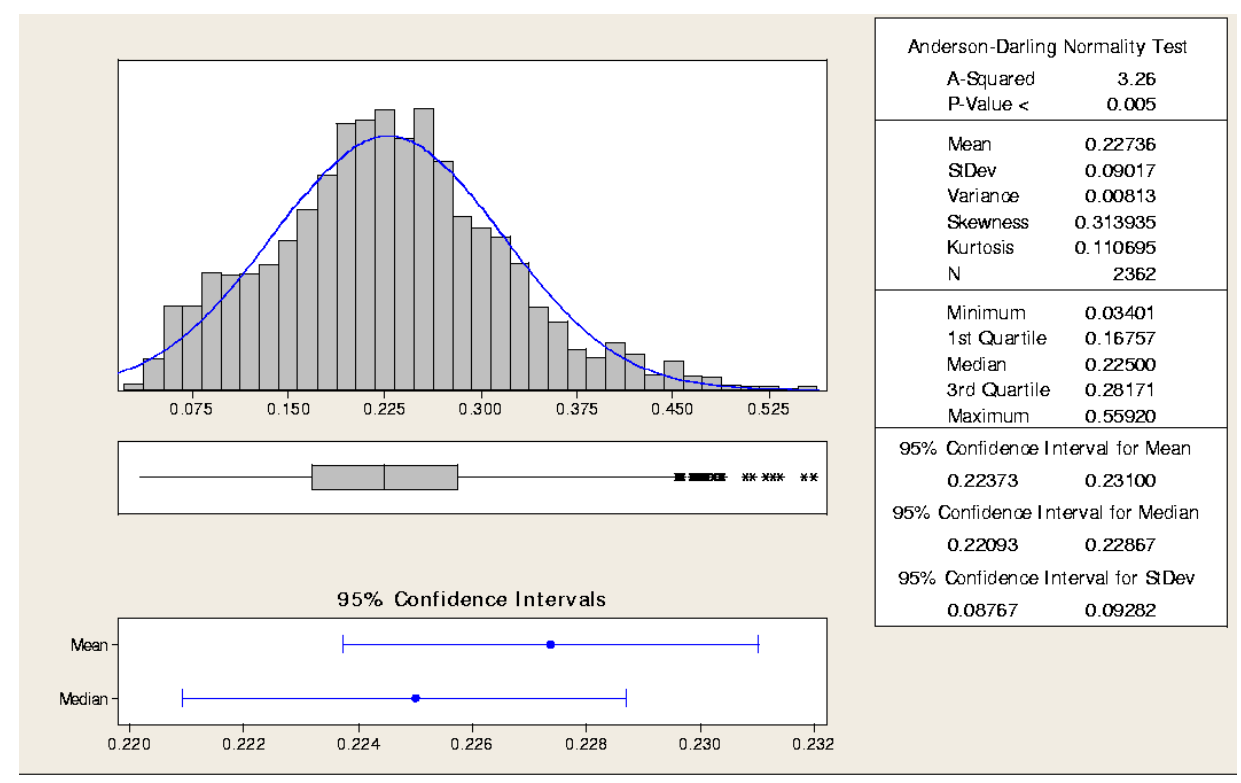

(b) Statistical analysis of porosity distribution

Figure 74: Porosity distribution of R145 

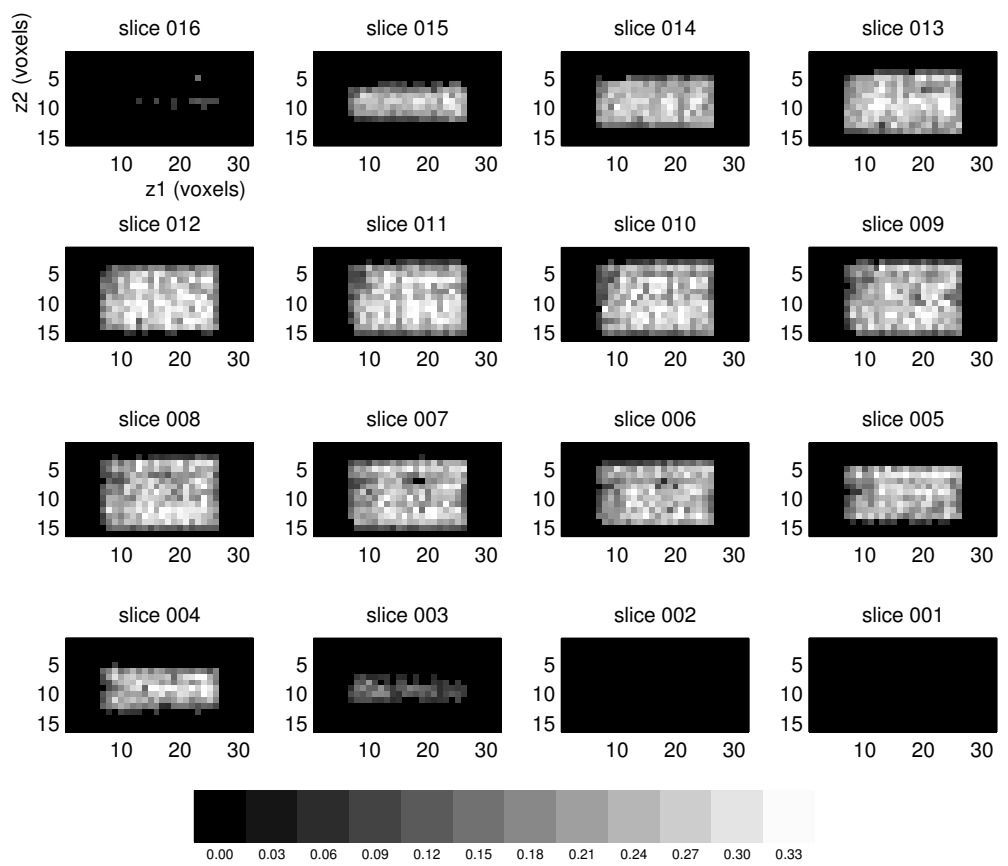

(a) Three-dimensional porosity image in gray scale

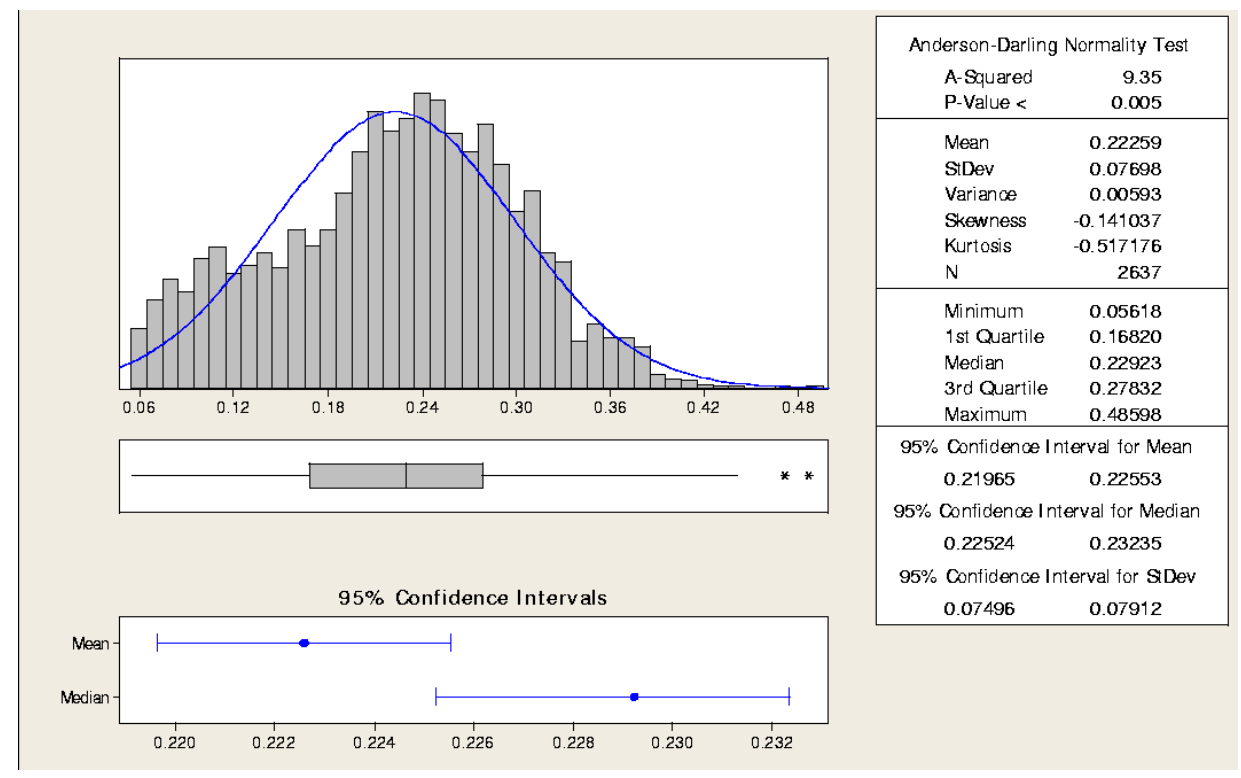

(b) Statistical analysis of porosity distribution

Figure 75: Porosity distribution of R207 

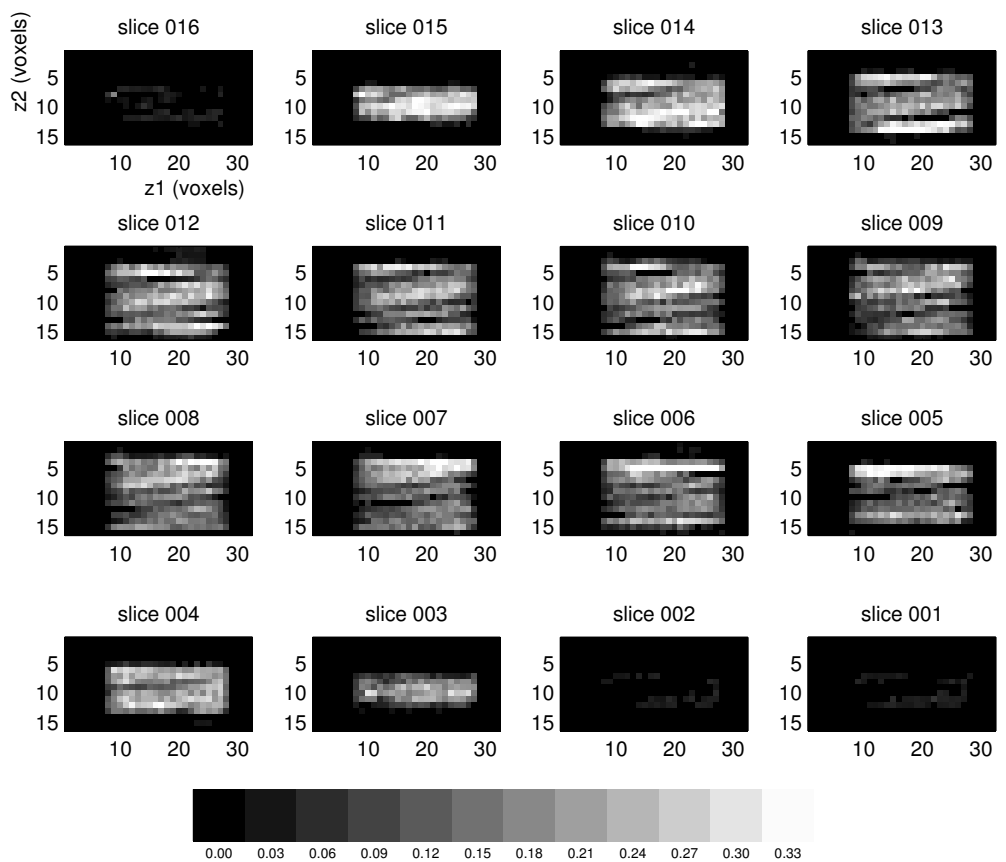

(a) Three-dimensional porosity image in gray scale

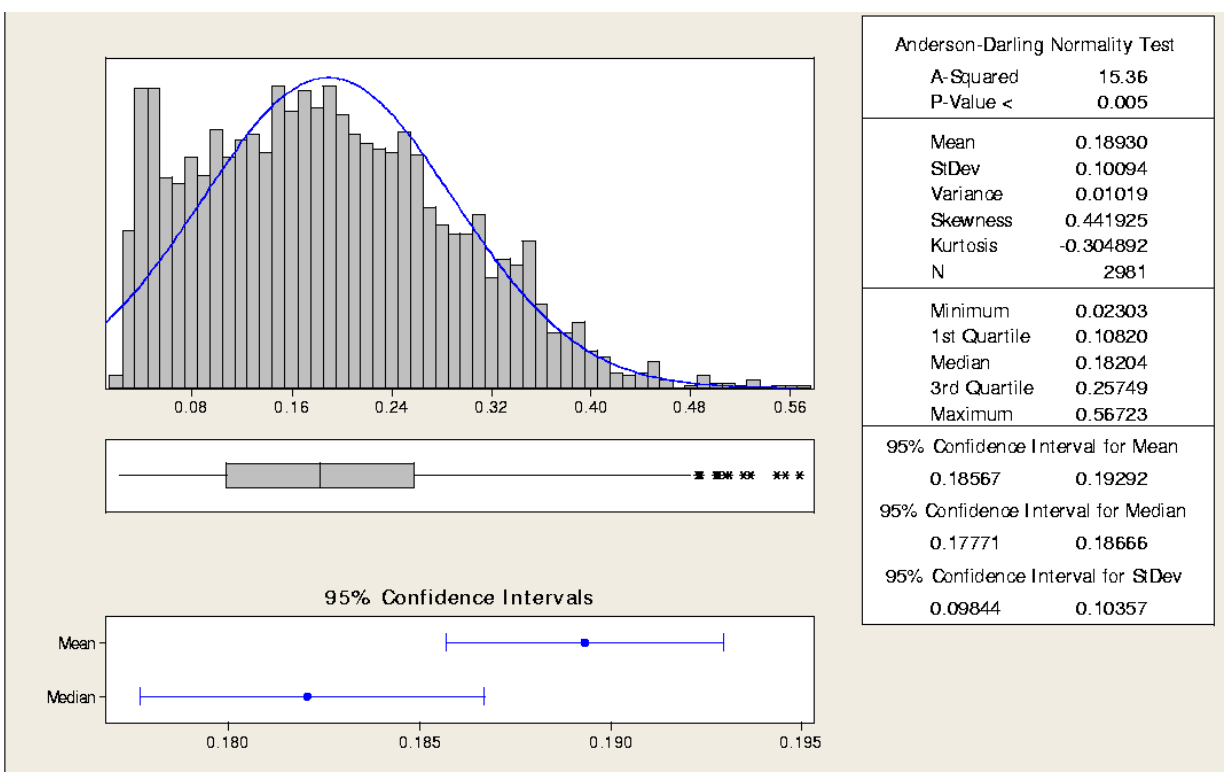

(b) Statistical analysis of porosity distribution

Figure 76: Porosity distribution of R221 

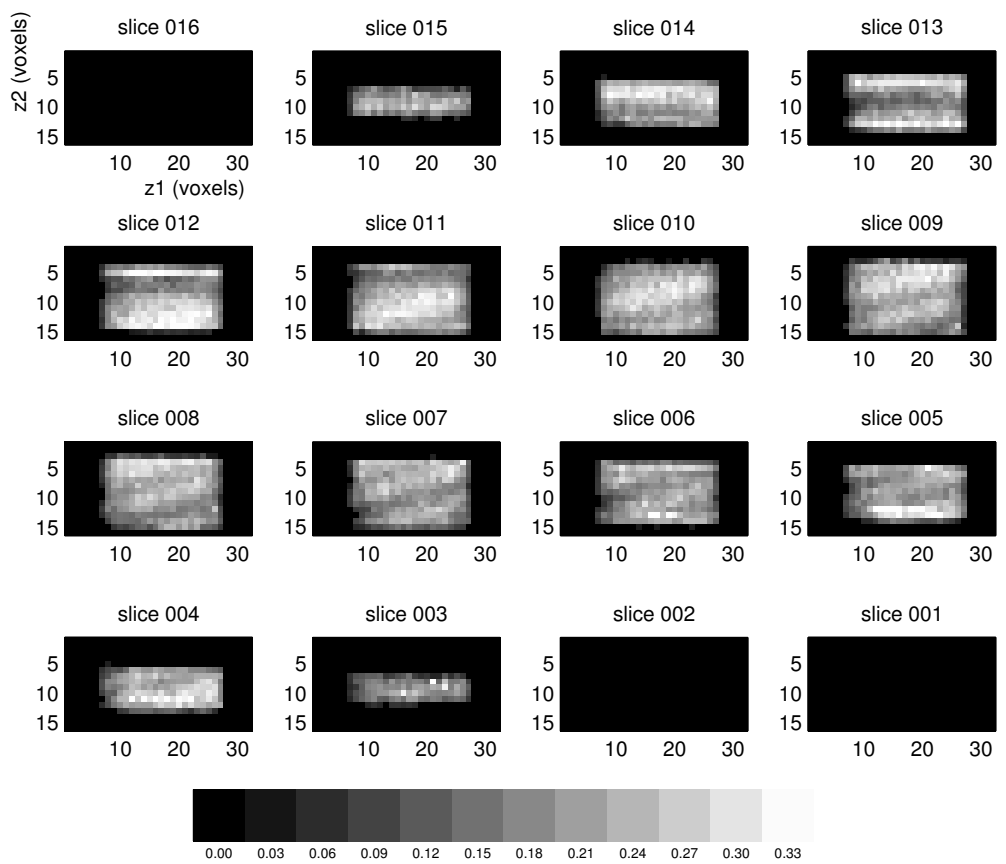

(a) Three-dimensional porosity image in gray scale

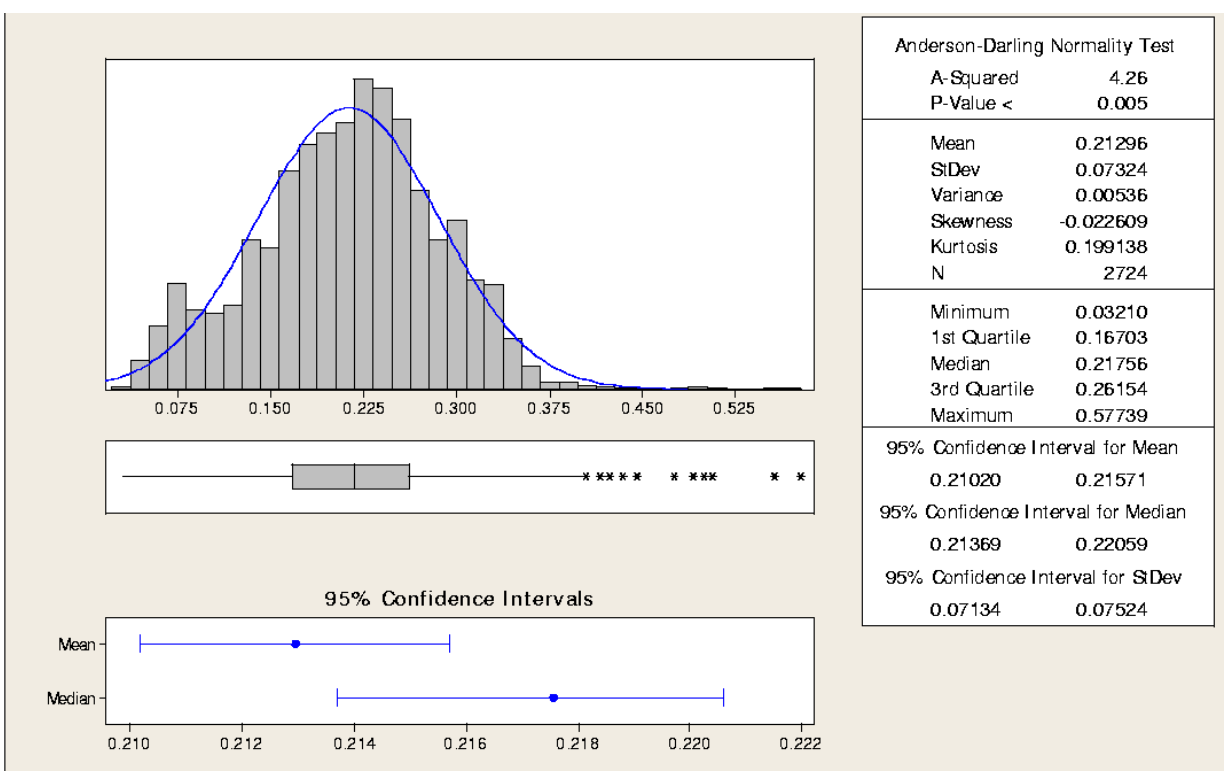

(b) Statistical analysis of porosity distribution

Figure 77: Porosity distribution of R237 


\section{Appendix 4. Flow Compensation}

In this section, we describe how the effect of fluid flow on spatial encoding is eliminated by "flow compensation". This section focus on the frequency encoding on the $z$-direction along which the fluid primarily flows. We assume that the effect of flow on the other two spatial encodings (phase encoding) is negligible.

The phase change $\varphi$ of a spin due to gradient fields applied during the time from $t_{1}$ to $t_{2}$ can be represented by

$$
\varphi=\int_{t_{1}}^{t_{2}} \gamma \mathbf{G}(t) \cdot \mathbf{r}(t) d t
$$

where $\gamma$ is gyromagnetic ratio, $\mathbf{G}(t)$ is the applied gradient field, and $\mathbf{r}(t)$ is the position vector at time $t$. Let us focus on only axial direction of the gradient and position vectors here. In general, a typical frequency encoding is composed of a defocusing gradient field and refocusing read-out gradient. The duration and strength of the defocusing gradient field is designed such that the total phase shift is zero at the center of the read-out gradient. If the fluid is stationary, the position of the spin is constant, i.e., $\mathbf{r}(t)=r_{0}$. Then, the defocusing gradient strength, grdp, is calculated as follows for trapezoidal shape of gradient fields: The phase change due to defocusing gradient is given by

$$
\begin{aligned}
& \int_{t_{1}^{*}}^{t_{1}^{*}+d 4} g r d p \cdot \frac{t-t_{1}^{*}}{d 4} r_{0} d t+\int_{t_{1}^{*}+d 4}^{t_{1}^{*}+d 4+d 11} g r d p r_{0} d t \\
+ & \int_{t_{1}^{*}+d 4+d 11}^{t_{1}^{*}+d 4+d 11+d 4} g r d p \cdot \frac{t_{1}^{*}+d 4+d 11+d 4-t}{d 4} r_{0} d t=g r d p(d 11+d 4) r_{0}
\end{aligned}
$$

Here, $t_{1}^{*}$ is the time of which the defocusing gradient begins, $d 4$ is rise time of the gradient, and $d 11$ is the duration of gradient. Gyromagnetic ratio is omitted for simplification in Eq. (130) and in the following. The phase change due to the the rephasing gradient, strength gro, up to the center of acquisition time, $a q q$, is

$$
\int_{t_{2}^{*}}^{t_{2}^{*}+d 4} g r o \cdot \frac{t-t_{2}^{*}}{d 4} r_{0} d t+\int_{t_{2}^{*}+d 4}^{t_{2}^{*}+d 4+a q q / 2} \text { gro } r_{0} d t=\frac{1}{2} \text { gro }(a q q+d 4) r_{0}
$$

Here, $t_{2}^{*}$ is the starting time of the read-out gradient field. The $g r d p$ which makes $(130)+(131)=$ 0 is

$$
\operatorname{grdp}=-\frac{\operatorname{gro}(a q q+d 4)}{2(d 11+d 4)}
$$

When the fluid flows with constant velocity of $v, r_{0}$ becomes $r\left(t_{1}^{*}\right)+\left(t-t_{1}^{*}\right) v$ and $r\left(t_{2}^{*}\right)+\left(t-t_{2}^{*}\right) v$ for defocusing and refocusing gradients, respectively. Suppose the defocusing gradient strength is 
adjusted from $g r d p$ to $g r d p+g r d p a$, the total integration of phase change from $t_{1}^{*}$ to $t_{2}^{*}+d 4+a q q / 2$ is expressed as

$$
\begin{aligned}
& \int_{t_{1}^{*}}^{t_{2}^{*}+d 4+a q q / 2} g(t) \cdot r(t) d t \\
= & \operatorname{grdpa}(d 4+d 11) r\left(t_{1}^{*}\right)+\frac{g r d p a v(d 4+d 11)(d 4+d 11+d 4)}{2} \\
+ & \operatorname{gro} \cdot v\left[\frac{d 4^{2}}{3}+\frac{a q q d 4}{2}+\frac{a q q^{2}}{8}+\frac{(a q q+d 4)\left[2\left(t_{2}^{*}-t_{1}^{*}\right)-(d 4+d 11+d 4)\right]}{4}\right]
\end{aligned}
$$

We can then choose grdpa such that Eq. (133) becomes zero, which means complete compensation. However, it is impossible to obtain the precise value of $v$ because it changes with respect to position. The problem can be easily solved by adding an additional gradient upon the first velocity encoding pulse.

Suppose the gradient strength of the first velocity encoding pulsed gradient is increased by modig. The phase change due to modig is

$$
\begin{aligned}
& \int_{t_{1}}^{t_{1}+d 4+d 11+d 4} \text { modig } g(t)\left[r\left(t_{1}\right)+\left(t-t_{1}\right) v\right] d t \\
= & \operatorname{modig}(d 4+d 11)\left[r\left(t_{1}\right)+\frac{(d 4+d 11+d 4)}{2} v\right],
\end{aligned}
$$

where $t_{1}$ is the starting time of the first velocity encoding gradient. After the two $90^{\circ}$ pulses, the phase of Eq. (134) is reversed. The total phase change is then calculated by the summation of Eqs. (133) and (134).

$$
\begin{aligned}
\int_{t_{1}}^{t_{1}^{*}+d 4+a q q / 2} g(t) \cdot r(t) d t & =-\operatorname{modig}(d 4+d 11) r\left(t_{1}\right)-\frac{\operatorname{modig} v(d 4+d 11)(d 4+d 11+d 4)}{2} \\
& +\operatorname{grdpa}(d 4+d 11) r\left(t_{1}^{*}\right)+\frac{\operatorname{grdpa} v(d 4+d 11)(d 4+d 11+d 4)}{2} \\
& +\operatorname{gro} \cdot v\left[\frac{d 4^{2}}{3}+\frac{a q q d 4}{2}+\frac{a q q^{2}}{8}+\frac{(a q q+d 4)\left[2\left(t_{2}^{*}-t_{1}^{*}\right)-(d 4+d 11+d 4)\right]}{4}\right]
\end{aligned}
$$

If we choose $g r d p a=$ modig and substitute $r\left(t_{1}^{*}\right)-r\left(t_{1}\right)=v \cdot \Delta$,

$$
\begin{aligned}
\int_{t_{1}}^{t_{1}^{*}+d 4+a q q / 2} g(t) \cdot r(t) d t & =\operatorname{modig}(d 4+d 11) v \Delta \\
& +\operatorname{gro} \cdot v\left[\frac{d 4^{2}}{3}+\frac{a q q d 4}{2}+\frac{a q q^{2}}{8}+\frac{(a q q+d 4)\left[2\left(t_{2}^{*}-t_{1}^{*}\right)-(d 4+d 11+d 4)\right]}{4}\right]
\end{aligned}
$$

The modig which satisfied Eq. (136) $=0$ is

$$
\text { modig }=\frac{- \text { gro }}{(d 4+d 11) \Delta}\left[\frac{d 4^{2}}{3}+\frac{a q q d 4}{2}+\frac{a q q^{2}}{8}+\frac{(a q q+d 4)\left[2\left(t_{2}^{*}-t_{1}^{*}\right)-(d 4+d 11+d 4)\right]}{4}\right]
$$

Here, $\left(t_{2}^{*}-t_{1}^{*}\right)-(d 4+d 11+d 4)$ is the time between the end of the second velocity encoding gradient pulse and the beginning of the read-out gradient. Note that modig does not include velocity $v$ because the corresponding term is canceled by the grdpa gradient at defocusing gradient. 